\title{
Integration of Metabolomics and Transcriptomics Reveals Ketone Body and Lipid Metabolism Disturbance Related to ER Stress in Liver
}

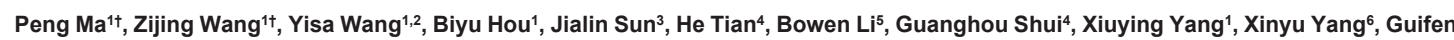

Qiang ${ }^{1, *}$, Chong Wee Liew ${ }^{7, *}$, Guanhua Du1,*

1 State Key Laboratory of Bioactive Substance and Function of Natural Medicines, Institute of Materia Medica, Chinese Academy of Medical Sciences and Peking Union Medical College and Beijing Key Laboratory of Drug Target and Screening Research, Beijing 100050, China 2 College of Pharmacy, Harbin University of Commerce, Harbin 510006, China

3 Department of Pharmacy, the Affiliated Hospital of Qingdao University, Qindao 266000, China

4 State Key Laboratory of Molecular Developmental Biology, Institute of Genetics and Developmental Biology, Chinese Academy of Sciences, Beijing 100101, China

5 LipidALL Technologies Ltd., Changzhou, China

6 Department of Pharmaceutical Analysis, College of Chemistry and Chemical Engineering, Queen's University Belfast, Belfast, BT7 1NN, United Kingdom

7 Department of Physiology and Biophysics, College of Medicine, University of Illinois at Chicago, Chicago, 60612 Illinois, USA

* Correspondence: Guifen Qiang, qianggf@imm.ac.cn; Chong Wee Liew, cwliew@uic.edu; Guanhua Du,dugh@imm.ac.cn.

† These authors contributed equally to this work

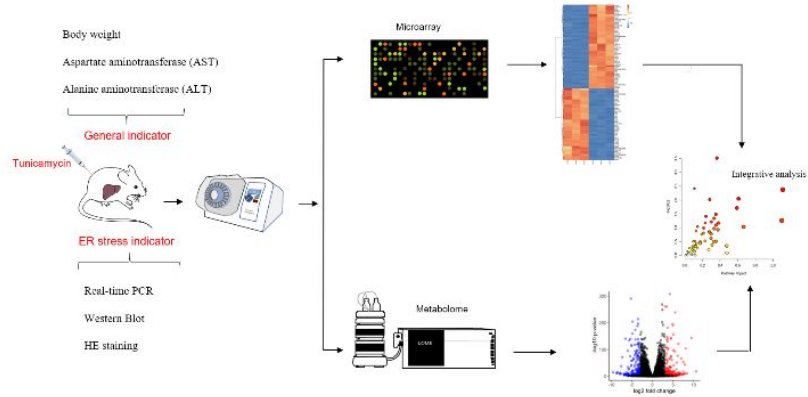

Figure S1 - Weighted correlation network analysis (WGCNA) power calculation

Figure S2 - Altered genes and metabolites in $\mathrm{C} 57 \mathrm{Bl} / 5 \mathrm{~N}$ mice after tunicamycin injection, shown in metabolic pathways.

Table S1 - Protein protein interaction network

Table S2 - WGCNA (Weighted correlation network analysis) network.

Table S3 - Core subnetworks of WGCNA network generated by MCODE plugin.

Table S4 - Core subnetworks of PPI network generated by MCODE plugin.

Table S5 - Differentially expressed classes of liver metabolites (normalized).

Table S6 - Differentially expressed glycerophosphoglycerols, glycerophosphocholines and glycerophosphoethanolamines (normalized).

Table S7 - Differentially expressed glycerophosphoglycerols, glycerophosphocholines and glycerophosphoethanolamines (normalized).

Table S8 - Metabolite set enrichment analysis. 

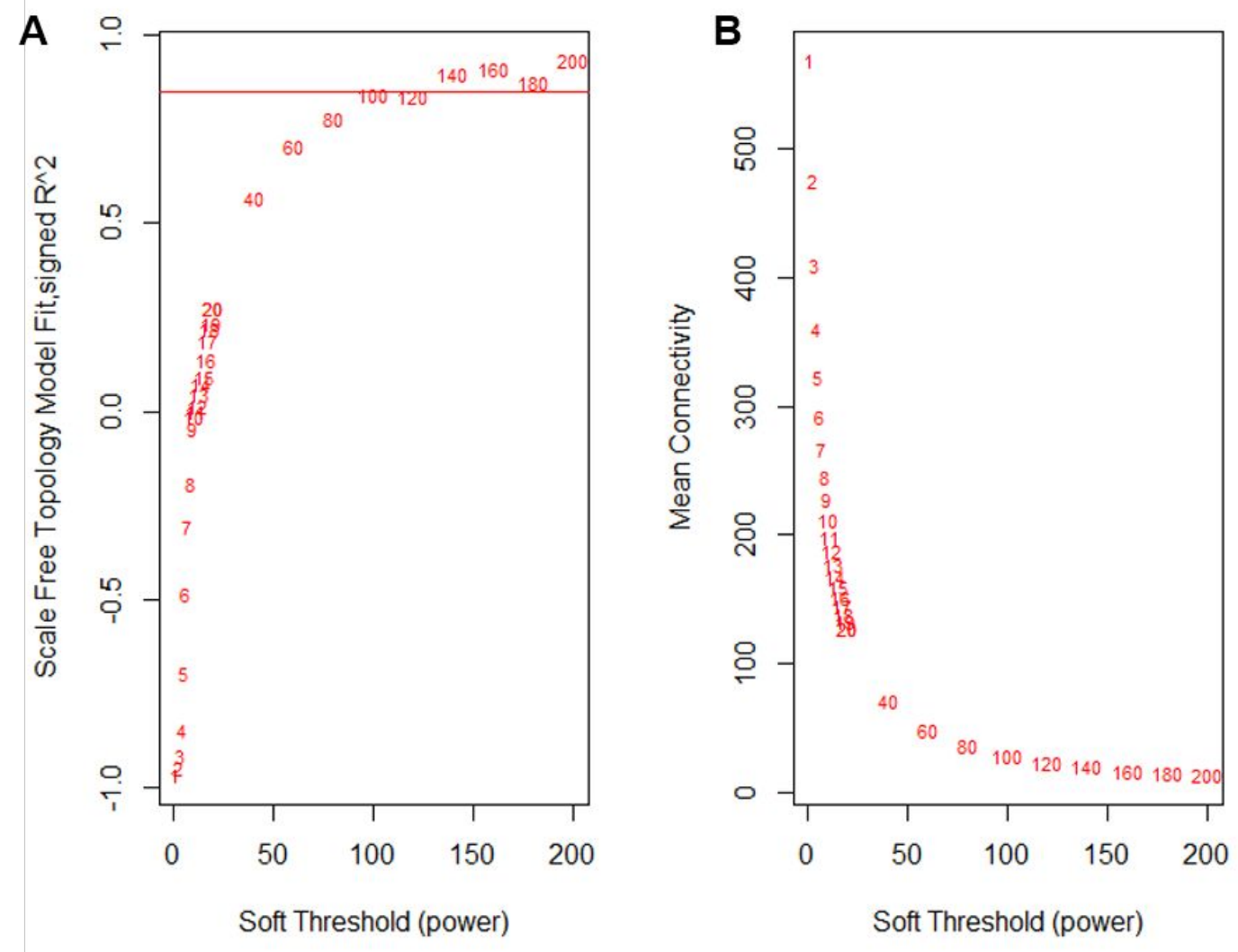

Figure S1 Weighted correlation network analysis (WGCNA) power calculation. (A) Scale free topology model fit calculation. (B) Mean connectivity calculation. 
A

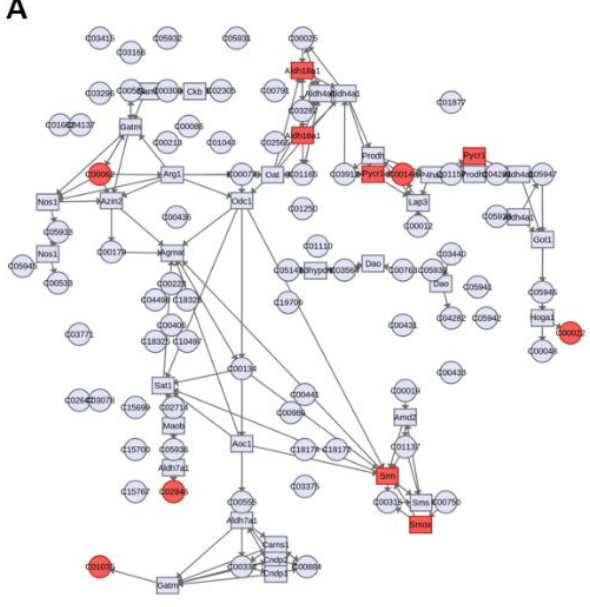

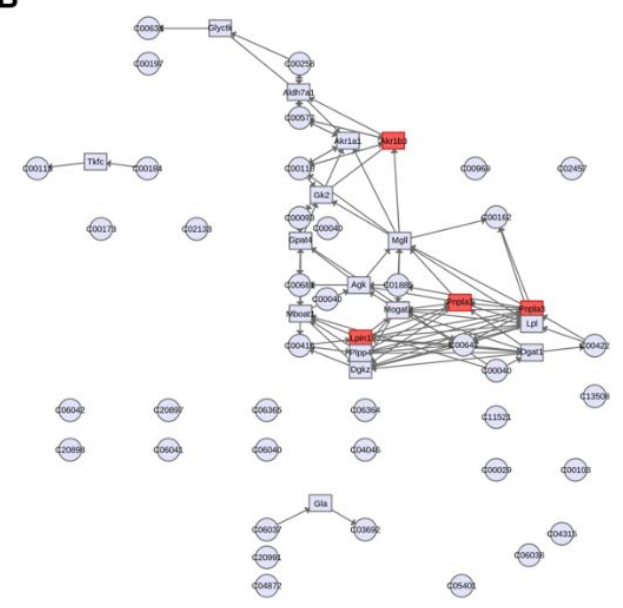

C

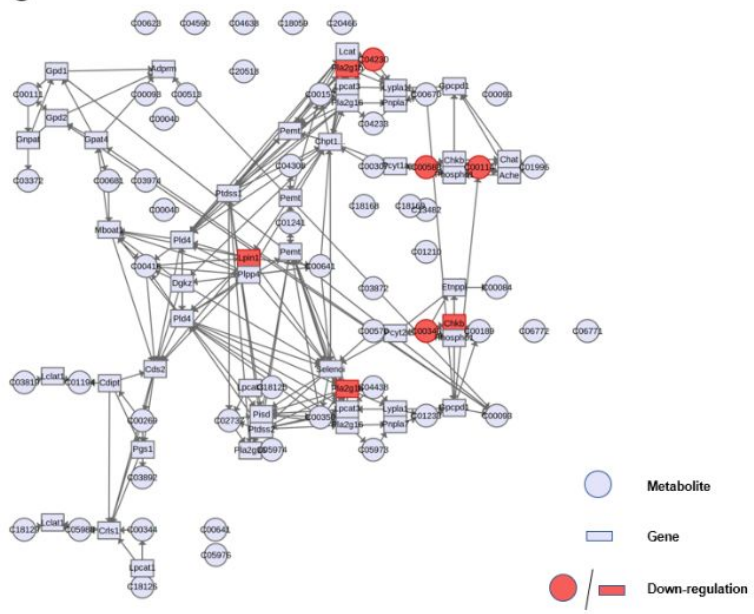

Figure S2 Altered genes and metabolites in C57B1/5N mice after tunicamycin injection, shown in metabolic pathways. (A) Arginine and proline metabolism pathway. (B) Glycerolipid metabolism pathway. (C) Glycerophospholipid metabolism pathway.

Table S1 Protein protein interaction network.

\begin{tabular}{cc}
\hline node1 & node2 \\
\hline Hspa5 & Hyou1 \\
\hline Hspa5 & Pdia4 \\
\hline Syvn1 & Sel11 \\
\hline Pdia4 & Hyou1 \\
\hline Gins2 & Cdc45 \\
\hline Nrg1 & Egfr \\
\hline Slc3a2 & Slc7a11 \\
\hline Abcg5 & Abcg8 \\
\hline Sel11 & Derl3
\end{tabular}




\begin{tabular}{|c|c|}
\hline Ccne2 & Cdkn1a \\
\hline Areg & Egfr \\
\hline Derl1 & Sel11 \\
\hline Manf & Creld2 \\
\hline Manf & Hspa5 \\
\hline Smox & Srm \\
\hline Hspa5 & Dnajc3 \\
\hline Creld2 & Sdf211 \\
\hline Derl1 & Derl3 \\
\hline Syvn1 & Derl1 \\
\hline Evc2 & Iqce \\
\hline Atf3 & Ddit3 \\
\hline Aldh112 & Mthfd2 \\
\hline Pycr1 & Aldh18a1 \\
\hline Wnt5a & Egfr \\
\hline Syce3 & $\operatorname{Rec} 8$ \\
\hline Hsd3b5 & Cyp7a1 \\
\hline Acacb & Acss 2 \\
\hline Hyou1 & Dnajc3 \\
\hline Hspa5 & Hspa1a \\
\hline Arfgap3 & Kdelr3 \\
\hline Tmem67 & Cep83 \\
\hline Cish & Asb2 \\
\hline Manf & Pdia4 \\
\hline Hspa5 & Sdf211 \\
\hline Pycr2 & Pycr1 \\
\hline Pycr2 & Aldh18a1 \\
\hline Gng13 & Gnat2 \\
\hline Wfs1 & Dnajc3 \\
\hline Pdia4 & Dnajc3 \\
\hline Hspa5 & Hspa13 \\
\hline Pdia4 & Sdf211 \\
\hline Ppargc1b & Srebf1 \\
\hline Hspa5 & Ddit3 \\
\hline Ppargcla & Nr1d1 \\
\hline Nop56 & Rrs1 \\
\hline Rock2 & Rnd1 \\
\hline Smox & Aoc2 \\
\hline Egfr & Ptk6 \\
\hline Acss 2 & Acat2 \\
\hline Dhfr & Aldh112 \\
\hline Lpin2 & Lpin3 \\
\hline
\end{tabular}




\begin{tabular}{|c|c|}
\hline Manf & Hyou1 \\
\hline Ppargcla & Prkab2 \\
\hline Sdr16c5 & Bcmo1 \\
\hline Wnt5a & Wnt9a \\
\hline Cnksr1 & Map2k1 \\
\hline Nrg1 & Ptk6 \\
\hline Cyp2b10 & Сур2c39 \\
\hline Ube $2 \mathrm{u}$ & Fbxl16 \\
\hline Hyou1 & Sdf211 \\
\hline Aox3 & Bcmo1 \\
\hline Gng13 & Adralb \\
\hline Hacl1 & Acot3 \\
\hline Etnk2 & Etnppl \\
\hline Pdia4 & Creld2 \\
\hline Dhcr7 & Cyp7a1 \\
\hline Gng13 & Mtnr1a \\
\hline Dhfr & Akr1b7 \\
\hline Apoa5 & Notum \\
\hline Acacb & Acat2 \\
\hline Cdkn1a & Ptk6 \\
\hline Prkab2 & Sesn3 \\
\hline Klkb1 & Gplbb \\
\hline Syvn1 & Derl3 \\
\hline Prlr & Cish \\
\hline Mtnrla & Sucnr1 \\
\hline Ube $2 \mathrm{u}$ & Asb2 \\
\hline Serpinb6a & Plaur \\
\hline Hspa1a & Hspa13 \\
\hline Cdkn1a & Myc \\
\hline $\mathrm{H} 2-\mathrm{Bl}$ & Hspa5 \\
\hline Kif22 & Kdelr3 \\
\hline P2ry4 & Sucnr1 \\
\hline Kif26b & Kif22 \\
\hline Arfgap3 & Kif22 \\
\hline Kif26b & Arfgap3 \\
\hline Aox3 & Sdr16c5 \\
\hline Adralb & Prok1 \\
\hline Rxfp2 & Adm2 \\
\hline Fbxl16 & Asb2 \\
\hline Wnt5a & Gnat2 \\
\hline Hspa5 & Dnajb9 \\
\hline Adamts7 & Sema5b \\
\hline
\end{tabular}




\begin{tabular}{|c|c|}
\hline Mtnr1a & Ptgdr2 \\
\hline Mtnrla & P2ry4 \\
\hline Gng13 & Rxfp2 \\
\hline Gng13 & Wnt5a \\
\hline Ptgdr2 & Gpsm2 \\
\hline $\mathrm{H} 2-\mathrm{B} 1$ & $\operatorname{Sec} 24 d$ \\
\hline Mtnrla & Gpsm2 \\
\hline Sec24d & Areg \\
\hline Apoa5 & Dnajc3 \\
\hline Gng13 & Prok1 \\
\hline Gng13 & Adm2 \\
\hline $\mathrm{H} 2-\mathrm{B} 1$ & Serpinb6a \\
\hline Rtp3 & Olfr1454 \\
\hline Kif26b & Kdelr3 \\
\hline Notum & Dnajc3 \\
\hline Gng13 & Gpsm2 \\
\hline Apoa5 & Wfs1 \\
\hline Ptgdr2 & Sucnr1 \\
\hline Notum & Wfs1 \\
\hline P2ry4 & Ptgdr2 \\
\hline Gng13 & P2ry4 \\
\hline P2ry4 & Gpsm2 \\
\hline mCG_19844 & Plaur \\
\hline Gpsm2 & Sucnr1 \\
\hline Cish & Fbxl16 \\
\hline Gng13 & Sucnr1 \\
\hline Gng13 & Ptgdr2 \\
\hline Taflc & Gatad2b \\
\hline Olfr102 & Rtp3 \\
\hline Hspa1a & Dnajc3 \\
\hline Aspg & Asns \\
\hline Manf & Dnajc3 \\
\hline Cyp2b10 & Cyp7a1 \\
\hline Hyou1 & Creld2 \\
\hline Syvn1 & Hspa5 \\
\hline Oasl1 & Dhx58 \\
\hline Slc10a1 & Cyp7a1 \\
\hline Trib3 & Ddit3 \\
\hline Abcg 5 & Cyp7a1 \\
\hline Acss 2 & Pck1 \\
\hline Manf & Sdf211 \\
\hline Asns & Psat1 \\
\hline
\end{tabular}




\begin{tabular}{|c|c|}
\hline Dbp & Nr1d1 \\
\hline Abcg8 & Cyp7a1 \\
\hline Myc & Egfr \\
\hline Tjp3 & Cldn1 \\
\hline Hist1h4c & Brdt \\
\hline Acacb & Prkab2 \\
\hline Gadd45a & Gadd $45 b$ \\
\hline Hspa5 & Derl3 \\
\hline Aldh18a1 & Psat1 \\
\hline Tgm1 & Lor \\
\hline Pnpla3 & Srebf1 \\
\hline Psat1 & Mthfd2 \\
\hline Hspa5 & Creld2 \\
\hline Oip5 & $\mathrm{Cdc} 45$ \\
\hline Msh3 & Slx $1 b$ \\
\hline Srd5a1 & Srd5a2 \\
\hline Acacb & Srebf1 \\
\hline Hsd3b5 & Сур2c39 \\
\hline Sel11 & Foxred2 \\
\hline Srd5a1 & Hsd3b5 \\
\hline Aldh18a1 & Mthfd2 \\
\hline Spdya & $\mathrm{Cdc} 25 \mathrm{c}$ \\
\hline Aox3 & Acss 2 \\
\hline Сур2b10 & Hsd3b5 \\
\hline Hspa5 & Sel11 \\
\hline Mpzl3 & Zfp750 \\
\hline Cyp7a1 & Сур2c39 \\
\hline Slc17a7 & Slc17a 8 \\
\hline Cdkn1a & $\mathrm{Cdc} 45$ \\
\hline Trmt61a & Rrs1 \\
\hline Hyou1 & Cdnf \\
\hline Hes6 & Foxa2 \\
\hline $\mathrm{Cdc} 25 \mathrm{c}$ & Kif22 \\
\hline Tmem67 & Nphp3 \\
\hline Pck1 & Srebf1 \\
\hline Gadd45a & Cdknla \\
\hline Ifi47 & Parp14 \\
\hline $\mathrm{Cpz}$ & Gabrb3 \\
\hline Parp14 & Dhx58 \\
\hline Fgf21 & Egfr \\
\hline Rgs 12 & Gpsm2 \\
\hline Cep295 & Sass6 \\
\hline
\end{tabular}




\begin{tabular}{|c|c|}
\hline Syvn1 & Hyou1 \\
\hline Nfxl1 & Dyx 1c1 \\
\hline Asns & Mthfd2 \\
\hline Rpl30 & Nop56 \\
\hline Slc20a1 & Slc34a3 \\
\hline Parp14 & Iigp1 \\
\hline Ifi47 & Iigp1 \\
\hline Notum & Wnt9a \\
\hline Wars & Lars \\
\hline Notum & Wnt5a \\
\hline Obfc1 & $\mathrm{Cdc} 45$ \\
\hline Slc10a1 & Abcg5 \\
\hline Figf & Egfr \\
\hline Hyou1 & Dnajb9 \\
\hline Creld2 & Derl3 \\
\hline Trib3 & Asns \\
\hline Srd5a2 & Hsd3b5 \\
\hline Apoa5 & Pla2g7 \\
\hline Fgf21 & Cyp7a1 \\
\hline Hspala & Dnajb9 \\
\hline Lpin2 & Pnpla3 \\
\hline Hyou1 & Sel11 \\
\hline Lpin3 & Pnpla3 \\
\hline Cyp2b10 & Aox3 \\
\hline Aox3 & Сур2c39 \\
\hline $\mathrm{Rd} 3$ & Suco \\
\hline Cyp7a1 & Srebf1 \\
\hline Dhfr & Mthfd2 \\
\hline Hspa5 & Derl1 \\
\hline Pdia4 & Derl3 \\
\hline Oip5 & Gins2 \\
\hline Aldh18a1 & Aldh112 \\
\hline Slc3a2 & Slc1a3 \\
\hline Gpsm2 & Fn3k \\
\hline Parp14 & Oasl1 \\
\hline Ppargc 1a & Pck1 \\
\hline Aldh8a1 & Aldh18a1 \\
\hline Slc10a1 & Abcg 8 \\
\hline Abcg 5 & Srebf1 \\
\hline Pard3b & Gpsm2 \\
\hline $\mathrm{Cdc} 25 \mathrm{c}$ & Ccne2 \\
\hline Cldn 2 & Cldn1 \\
\hline
\end{tabular}




\begin{tabular}{|c|c|}
\hline Cdnf & Dnajc3 \\
\hline Ung & Dhfr \\
\hline Msh3 & Ung \\
\hline Ccdc116 & Ccde134 \\
\hline Hspa13 & Dnajb9 \\
\hline Gins2 & Kif22 \\
\hline Asns & Atf3 \\
\hline Ccdc151 & Dnaic1 \\
\hline Dnaic1 & Dyx1c1 \\
\hline Unc93a & Mfsd9 \\
\hline $\mathrm{Nrg} 1$ & Areg \\
\hline Drc1 & Dyx1c1 \\
\hline Klkb1 & Proz \\
\hline Сyp2b23 & Hsd3b5 \\
\hline Slc17a7 & Slc1a3 \\
\hline Hspa5 & Cdnf \\
\hline Cyp7a1 & Pck1 \\
\hline Isg20 & Oasl1 \\
\hline Pla2g4f & Pla2g7 \\
\hline Hyou1 & $\operatorname{Sec} 24 d$ \\
\hline Cdnf & Creld2 \\
\hline Mpdz & Cldn1 \\
\hline $\mathrm{Cdc} 25 \mathrm{c}$ & Cdkn1a \\
\hline Pla2g4f & Сур2c39 \\
\hline Ccne2 & Myc \\
\hline Trib3 & Atf3 \\
\hline Cyp2b23 & Cyp7a1 \\
\hline Nop56 & Trmt61a \\
\hline $\mathrm{Cdc} 25 \mathrm{c}$ & $\mathrm{Cdc} 45$ \\
\hline Dhcr7 & Acat2 \\
\hline Klkb1 & Acpp \\
\hline Sorl1 & Psen 2 \\
\hline Apoa5 & Srebf1 \\
\hline Cldn14 & Cldn2 \\
\hline Hspa5 & Myc \\
\hline Smpd3 & mCG_19844 \\
\hline Myc & Map2k1 \\
\hline Abcg8 & Srebf1 \\
\hline Slc26a2 & $\mathrm{Cdc} 45$ \\
\hline Ddit3 & Dnajc3 \\
\hline Сyp2b23 & Сур2c39 \\
\hline Cyp2b23 & Aox3 \\
\hline
\end{tabular}




\begin{tabular}{|c|c|}
\hline Fgf 21 & Pck1 \\
\hline Oip5 & Kif22 \\
\hline Grb14 & Srebf1 \\
\hline Slc7a11 & Slc1a3 \\
\hline Gadd45a & Ddit3 \\
\hline Sdf211 & Derl3 \\
\hline Cldn2 & Tjp3 \\
\hline Nmnat3 & Aldh18a1 \\
\hline Myc & Srebf1 \\
\hline Apoa5 & Abcg5 \\
\hline Syvn1 & Creld2 \\
\hline Сур2b10 & Pla2g4f \\
\hline Hspa5 & Sec24d \\
\hline Сyp2b23 & $\mathrm{Pla} 2 \mathrm{~g} 4 \mathrm{f}$ \\
\hline Ddit3 & Dnajb9 \\
\hline Rpl30 & Rrs1 \\
\hline Rtkn2 & Sdr16c5 \\
\hline Apoa5 & Sorl1 \\
\hline Arfgap3 & Rbm15b \\
\hline Fgf21 & Srebf1 \\
\hline Frmd3 & Psat1 \\
\hline Selenbp2 & Plaur \\
\hline Cldn14 & Cldn1 \\
\hline Aen & Rrs 1 \\
\hline Syvn1 & Pdia4 \\
\hline Kif22 & Fam83d \\
\hline Gadd45a & Atf3 \\
\hline Ppargcla & Srebf1 \\
\hline Wars & Sntg2 \\
\hline Aspg & Aldh18a1 \\
\hline Hsd3b5 & Keg1 \\
\hline Dnaic1 & Drc1 \\
\hline Golt1b & Aldh18a1 \\
\hline Nmnat3 & Parp14 \\
\hline Sec24d & Kdelr3 \\
\hline Tmtc4 & Gfra2 \\
\hline Pdia4 & Dnajb9 \\
\hline Ppargcla & Fgf21 \\
\hline Aspg & Sds \\
\hline Ccdc151 & Dlgap3 \\
\hline Slc26a2 & Slc13a4 \\
\hline Mfsd9 & Mfsd7a \\
\hline
\end{tabular}




\begin{tabular}{|c|c|}
\hline Rrs1 & Isg20 \\
\hline Gadd45a & Myc \\
\hline Ccne2 & $\mathrm{Cdc} 45$ \\
\hline Pdia4 & Ddit3 \\
\hline Havcr2 & Vsir \\
\hline Manf & Foxred2 \\
\hline Tmem88b & Fam81a \\
\hline Syvn1 & Dnajc3 \\
\hline Cebpe & Tfec \\
\hline Gmds & Nans \\
\hline Slc10a1 & Slc51b \\
\hline Etnk2 & Golt1b \\
\hline Oip5 & $\mathrm{Cdc} 25 \mathrm{c}$ \\
\hline Gng13 & Olfr128 \\
\hline Trmt61a & Srm \\
\hline Aim11 & Zfp280c \\
\hline Cnot6 & Gadd45b \\
\hline $\operatorname{Rad} 54 b$ & $\mathrm{Cdc} 45$ \\
\hline Foxa2 & Myc \\
\hline Esrp1 & Myc \\
\hline Atf3 & Gadd $45 b$ \\
\hline Arfgap3 & Sntg2 \\
\hline Gng13 & Olfr102 \\
\hline Gng13 & Prkab2 \\
\hline Gng13 & Olfr934 \\
\hline Gng13 & Olfr1454 \\
\hline Gng13 & Olfr1477 \\
\hline Gng13 & Olfr1043 \\
\hline Gng13 & Olfr214 \\
\hline Hspa5 & Atf3 \\
\hline Thrsp & Srebf1 \\
\hline Slc26a2 & Slc13a2 \\
\hline Dnajc3 & Derl3 \\
\hline Asns & Ddit3 \\
\hline Myc & Lgr5 \\
\hline Gng13 & Btg3 \\
\hline Cdkn1a & Gadd $45 b$ \\
\hline Cdkn1a & Egfr \\
\hline Aldh18a1 & Impa2 \\
\hline Manf & Syvn1 \\
\hline Pla2g4f & Nrn11 \\
\hline Manf & Dnajb9 \\
\hline
\end{tabular}




\begin{tabular}{|c|c|}
\hline Egfr & Plaur \\
\hline Cidec & Srebf1 \\
\hline Pdia4 & Fkbp11 \\
\hline Adap1 & Gpr146 \\
\hline Dhcr7 & Abcg5 \\
\hline Aldh18a1 & Srm \\
\hline Hyou1 & Derl3 \\
\hline Trim66 & Galnt15 \\
\hline Dlgap3 & Rgs12 \\
\hline Nop56 & Lars \\
\hline Slc17a8 & Slc1a3 \\
\hline Hspa5 & Gadd45a \\
\hline Apoa5 & $\operatorname{Abcg} 8$ \\
\hline Slc51b & Cyp7a1 \\
\hline Cldn2 & Egfr \\
\hline Hal & Apoa5 \\
\hline Ccdc116 & Fam175a \\
\hline Zbtb7c & Srebf1 \\
\hline Hsd3b5 & Egfr \\
\hline Kif22 & $\mathrm{Cdc} 45$ \\
\hline Ifi47 & Oasl1 \\
\hline Slc3a2 & Slc36a1 \\
\hline $\operatorname{Rad} 54 b$ & Kif22 \\
\hline Hspa5 & Trib3 \\
\hline Gins2 & Ung \\
\hline Abcg 5 & Atp8b1 \\
\hline Bhmt & Hacl1 \\
\hline Hs3st6 & Necab1 \\
\hline Gnat2 & Rgs16 \\
\hline Timd2 & Scara5 \\
\hline Bdh1 & B3gat2 \\
\hline Derl1 & Dnajc3 \\
\hline Pycr1 & Psat1 \\
\hline Fgf21 & Bmp8b \\
\hline P2ry2 & Tfec \\
\hline Brdt & Ddah1 \\
\hline Pycr1 & Mthfd2 \\
\hline Brdt & Myc \\
\hline Dnajc3 & Sel11 \\
\hline Apoa5 & Cyp7a1 \\
\hline Upp2 & B3gat2 \\
\hline Slc7a11 & Egfr \\
\hline
\end{tabular}




\begin{tabular}{|c|c|}
\hline Slc36a1 & Slc1a3 \\
\hline Nipal3 & Taflc \\
\hline Gas7 & Gmds \\
\hline Pus3 & Thumpd3 \\
\hline Sorl1 & Plaur \\
\hline Rxfp2 & Atp8b1 \\
\hline Epb4.115 & Smox \\
\hline Cebpe & P2ry2 \\
\hline Acacb & Pck1 \\
\hline Myc & Dhfr \\
\hline Atf3 & Myc \\
\hline Nipsnap3a & Nans \\
\hline Ppargcla & Cyp7a1 \\
\hline Hspa5 & Srebf1 \\
\hline Nipal3 & Slc41a3 \\
\hline Hyou1 & Derl1 \\
\hline Dex & Slc1a3 \\
\hline Msh3 & Dhfr \\
\hline Dhcr7 & Igfals \\
\hline Sorl1 & Pla2g7 \\
\hline Sorl1 & Rock2 \\
\hline Wnt5a & Myc \\
\hline Wnt5a & Celsr1 \\
\hline Meox 1 & Foxa2 \\
\hline Rnf186 & Slc25a45 \\
\hline Rpl30 & Oasl1 \\
\hline Ccdc134 & Usp43 \\
\hline Aldh8a1 & Proz \\
\hline Asns & Aldh18a1 \\
\hline Sds & Aldh18a1 \\
\hline Dnajc3 & Fkbp11 \\
\hline Epb4.115 & Plbd2 \\
\hline Trib3 & Nupr1 \\
\hline Cidec & Pck1 \\
\hline Upp2 & Sds \\
\hline Hist1h4c & Ms1312 \\
\hline $\operatorname{Abcg} 8$ & Atp8b1 \\
\hline Slc17a7 & Slc30a3 \\
\hline Aldh18a1 & Dhfr \\
\hline Etnk2 & Ggt6 \\
\hline Rock2 & Egfr \\
\hline Mest & Zfp57 \\
\hline
\end{tabular}




\begin{tabular}{|c|c|}
\hline Trmt61a & Pus3 \\
\hline Nop56 & Myc \\
\hline Ptges31 & Aoc2 \\
\hline Zfp750 & Fn3k \\
\hline Lrit1 & Aoc2 \\
\hline Gfpt1 & Aldh18a1 \\
\hline Zbtb32 & ENSMUSG00000036027 \\
\hline Nphp3 & Prkd1 \\
\hline $\mathrm{Cdc} 25 \mathrm{c}$ & $\mathrm{Cdc} 14 \mathrm{~b}$ \\
\hline Sapcd2 & Fam83d \\
\hline Cyb5r1 & Mthfd2 \\
\hline $\mathrm{Kbp}$ & Prok1 \\
\hline Pdzk1ip1 & Map10 \\
\hline Dbp & Cyp7a1 \\
\hline Wdr17 & Dclk3 \\
\hline Acacb & Ncald \\
\hline Creld2 & Dnajc3 \\
\hline $\operatorname{Rad} 54 b$ & $\mathrm{Cdc} 25 \mathrm{c}$ \\
\hline Thrsp & Car14 \\
\hline Ppip5k1 & Dhx 58 \\
\hline Mpzl3 & Hsf5 \\
\hline Htra4 & Naa30 \\
\hline Pmm1 & Pbld1 \\
\hline Aox3 & Aldh18a1 \\
\hline Gfpt1 & Asns \\
\hline Slc10a1 & Atp8b1 \\
\hline $\mathrm{Cdc} 25 \mathrm{c}$ & Gadd45a \\
\hline Cdc14b & Fam81a \\
\hline Amigo3 & Creld2 \\
\hline Pllp & Plaur \\
\hline Sys 1 & Pdzk1ip1 \\
\hline Gadd45a & Trib3 \\
\hline Sys1 & Sec24d \\
\hline Dex & Slc17a7 \\
\hline Hspa5 & Egfr \\
\hline Hspala & Atf3 \\
\hline Oip5 & Fam83d \\
\hline Cldn1 & Egfr \\
\hline $\mathrm{Cdc} 25 \mathrm{c}$ & Gins2 \\
\hline $\mathrm{Cdc} 25 \mathrm{c}$ & Myc \\
\hline $\mathrm{Cdc} 25 \mathrm{c}$ & Fam83d \\
\hline Sdf211 & Dnajc3 \\
\hline
\end{tabular}




\begin{tabular}{|c|c|}
\hline Acss 2 & Srebf1 \\
\hline Hspa5 & Wfs1 \\
\hline Cyp2d12 & Ces1f \\
\hline Slc10a1 & Cyp2b10 \\
\hline Sfmbt2 & Fam81a \\
\hline Agpat9 & Dhx58 \\
\hline Syvn1 & Dnajb9 \\
\hline Cyb5r1 & Aldh112 \\
\hline Upp2 & Dhfr \\
\hline Hal & Sds \\
\hline Cish & Egfr \\
\hline Dnajc3 & Dnajb9 \\
\hline Asns & Pycr1 \\
\hline Bdh1 & Upp2 \\
\hline Trib3 & Mthfd2 \\
\hline Mapk15 & Map2k1 \\
\hline Hyou1 & Ddit3 \\
\hline $\mathrm{Cdc} 25 \mathrm{c}$ & Egfr \\
\hline Pnpla3 & Ces1d \\
\hline Prr16 & Tnfaip813 \\
\hline Fgf21 & Cidec \\
\hline Manf & Derl3 \\
\hline Ung & Aldh18a1 \\
\hline Bco2 & ENSMUSG00000036027 \\
\hline Hspa5 & Asns \\
\hline Methig1 & Cox6a2 \\
\hline Ccdc134 & Cep83 \\
\hline Sdf211 & Fkbp11 \\
\hline Ddit3 & Cdkn1a \\
\hline Thrsp & Acss2 \\
\hline Dclk3 & Dex \\
\hline Dhfr & $\mathrm{Cdc} 45$ \\
\hline Pdia4 & Sel11 \\
\hline Cnot6 & Actrt3 \\
\hline Asns & Slc7a11 \\
\hline Ccdc151 & Drcl \\
\hline Nr1d1 & Fgf21 \\
\hline Foxq1 & Gmds \\
\hline Spata2l & Upp2 \\
\hline Tes & Serpinbla \\
\hline Serpinb1a & Prtn3 \\
\hline Pycr2 & Psat1 \\
\hline
\end{tabular}




\begin{tabular}{|c|c|}
\hline Unc93a & Mfsd7a \\
\hline Wnt5a & Foxa2 \\
\hline Gpsm2 & Rgs16 \\
\hline Manf & Kdelr3 \\
\hline Hspala & Vsir \\
\hline Klkb1 & Aldh8a1 \\
\hline Prlr & Thrsp \\
\hline Egfr & Map2k1 \\
\hline Dnajc3 & Kdelr3 \\
\hline Cyp2b10 & B3gat2 \\
\hline Sec24d & Dnajc3 \\
\hline Creld2 & Kdelr3 \\
\hline Rpl30 & Lars \\
\hline Kbtbd11 & Zfp57 \\
\hline Olig1 & Slc1a3 \\
\hline Aldh8a1 & Rgn \\
\hline Olfml1 & Guca2a \\
\hline Cyp2d12 & Ces1d \\
\hline Esrp1 & Cldn1 \\
\hline Pdlim3 & Actn3 \\
\hline Ncald & Frmd3 \\
\hline Igfals & Keg1 \\
\hline Wnt5a & Lgr5 \\
\hline Atf3 & Cdkn1a \\
\hline Trib3 & Prkab2 \\
\hline Pdia4 & Derl1 \\
\hline Sys 1 & Naa30 \\
\hline Cnot6 & Tada2b \\
\hline Ifi47 & Dhx 58 \\
\hline Slc17a9 & Panx3 \\
\hline Hyou1 & Fkbp11 \\
\hline Slc10a1 & Pck1 \\
\hline Dclk3 & Ggt6 \\
\hline Cyp7a1 & Acat2 \\
\hline Cyb5r1 & Aldh18a1 \\
\hline Dhcr7 & Acss 2 \\
\hline Isg20 & Dhx58 \\
\hline Thrsp & Pck1 \\
\hline Gas7 & Cdc14b \\
\hline Ddit3 & Srebf1 \\
\hline Spata21 & Acot3 \\
\hline Msh3 & Obfc1 \\
\hline
\end{tabular}




\begin{tabular}{|c|c|}
\hline Usf2 & Adamts 7 \\
\hline Ppargcla & Acacb \\
\hline $\operatorname{Rad} 54 \mathrm{~b}$ & Gins2 \\
\hline Gpr21 & Mrgprf \\
\hline Cish & Igfals \\
\hline Syvn1 & Wfs1 \\
\hline ENSMUSG00000050538 & Olfml1 \\
\hline Ddit3 & Myc \\
\hline Brdt & Tada2b \\
\hline Bhmt & Dhfr \\
\hline Agpat9 & Srebfl \\
\hline Hspala & Ddit3 \\
\hline Lgr5 & Egfr \\
\hline Col4a5 & Lama3 \\
\hline Ccdc134 & Fam175a \\
\hline Ccne2 & Aldh18a1 \\
\hline Foxa2 & Pck1 \\
\hline Dhcr7 & Pck1 \\
\hline Nxpe2 & Rab17 \\
\hline Sds & Pck1 \\
\hline Acy3 & Pmm1 \\
\hline Lars & Asns \\
\hline Sdf211 & Kdelr3 \\
\hline Ces1d & Pck1 \\
\hline Hspa5 & Kdelr3 \\
\hline Nop56 & Adck3 \\
\hline Bves & Arhgef4 \\
\hline Hspa5 & Bik \\
\hline Trib3 & Adck3 \\
\hline Lpin2 & Srebf1 \\
\hline Car14 & Pllp \\
\hline Dhcr7 & Proz \\
\hline Usf2 & Myc \\
\hline $\mathrm{Rd} 3$ & Sel11 \\
\hline Cep295 & Col4a5 \\
\hline Hyou1 & Aldh18a1 \\
\hline Tnfrsf19 & Lgr5 \\
\hline Creld2 & Steap1 \\
\hline Dnajb9 & Derl3 \\
\hline Aen & Trmt61a \\
\hline Slx $1 b$ & Rad54b \\
\hline Ddit3 & Derl3 \\
\hline
\end{tabular}




\begin{tabular}{|c|c|}
\hline Spata21 & Cxxc5 \\
\hline Ptrh1 & Cyb5r1 \\
\hline Ppargclb & Pck1 \\
\hline Atf3 & Tnfrsf12a \\
\hline Nupr1 & Ddit3 \\
\hline Lyrm7 & Thumpd3 \\
\hline Fgf21 & Tnfrsf12a \\
\hline Apoa5 & Rgn \\
\hline Lars & Aldh18a1 \\
\hline Bhmt & Mthfd2 \\
\hline Srd5a1 & Dhcr7 \\
\hline Ifi47 & Isg20 \\
\hline Ptgdr2 & Areg \\
\hline Sdf211 & Dnajb9 \\
\hline Sel11 & Dnajb9 \\
\hline Hspa13 & Dnajc3 \\
\hline Gnat2 & Gpsm2 \\
\hline Ung & $\mathrm{Cdc} 45$ \\
\hline Foxa2 & Cyp7a1 \\
\hline Pdia4 & Sec24d \\
\hline Phlda1 & Tnfrsf19 \\
\hline Esrp1 & Prss8 \\
\hline Trib3 & Dnajc3 \\
\hline Epb4.115 & Pard3b \\
\hline Zfp750 & Lor \\
\hline Evc2 & Clstn1 \\
\hline Epb4.115 & Esrp1 \\
\hline Cdkn1a & Map2k1 \\
\hline Gplbb & Ica1 \\
\hline Gal3st1 & Pllp \\
\hline Slc25a53 & Slc25a42 \\
\hline Spdya & Rec8 \\
\hline Cish & Myc \\
\hline $\operatorname{Rad54b}$ & Obfc1 \\
\hline Kif26b & Fam81a \\
\hline Nans & Pmm1 \\
\hline Ccdc151 & Dyx1c1 \\
\hline Trib3 & Fosb \\
\hline Mov10 & mCG_19844 \\
\hline Slc10a1 & Srd5a1 \\
\hline Srd5a1 & Cish \\
\hline Abcg5 & Acat2 \\
\hline
\end{tabular}




\begin{tabular}{|c|c|}
\hline Slc7a11 & Psat1 \\
\hline Slc17a7 & Gabrb3 \\
\hline Grtp1 & Cdc14b \\
\hline Cdc14b & Rec8 \\
\hline Adralb & Serpini1 \\
\hline Fermt1 & Tmtc4 \\
\hline Adra1b & Chrna4 \\
\hline Mtnrla & Aoc2 \\
\hline Pdzk1ip1 & Vwce \\
\hline Oas11 & Usp43 \\
\hline Atf3 & Egfr \\
\hline Acacb & Thrsp \\
\hline Dhcr7 & Srebf1 \\
\hline Pdlim3 & Lims2 \\
\hline Mov10 & Dhx 58 \\
\hline Ccdc116 & Lpin3 \\
\hline Proz & Rgn \\
\hline Tnfrsf19 & Tnfrsf12a \\
\hline Acacb & $A \cot 3$ \\
\hline Pdzk1ip1 & Acy3 \\
\hline Abcg 5 & Bcmol \\
\hline Lama3 & Hsd3b5 \\
\hline Egfr & Wnt9a \\
\hline Ngef & Arhgef4 \\
\hline Scgb1c1 & Slc13a4 \\
\hline Acss 2 & Aldh18a1 \\
\hline Bhmt & Sds \\
\hline Apoa5 & Aldh8a1 \\
\hline Rock2 & Rnd2 \\
\hline Trmt61a & Isg20 \\
\hline Clstn1 & Serpini1 \\
\hline Bco2 & Aldh8a1 \\
\hline Ccne2 & Egfr \\
\hline Dhcr7 & Abcg8 \\
\hline Zfp322a & Cxxc5 \\
\hline Obfc1 & Ung \\
\hline Hspa1a & Egfr \\
\hline Rpl30 & Cox6a2 \\
\hline Msh3 & $\operatorname{Rec} 8$ \\
\hline Nrip2 & Ovgp1 \\
\hline Arhgef4 & Rnd1 \\
\hline Acacb & Ppargc 1b \\
\hline
\end{tabular}




\begin{tabular}{|c|c|}
\hline Bdh1 & Acss 2 \\
\hline Wars & Tyw1 \\
\hline Agpat9 & Kdelr3 \\
\hline Pycr1 & Aldh112 \\
\hline Cidec & Agpat9 \\
\hline Areg & Map2k1 \\
\hline P2ry4 & Panx3 \\
\hline Gfpt1 & Gmds \\
\hline Gins2 & Ccne2 \\
\hline Pnpla3 & Cidec \\
\hline Gnat2 & $\operatorname{Rgs} 12$ \\
\hline Ddit3 & Gadd45b \\
\hline Dhcr7 & Hsd3b5 \\
\hline Ptges 31 & Cyp2c39 \\
\hline Fam110c & Sntg2 \\
\hline Slc $44 a 3$ & Slc13a2 \\
\hline Trib3 & Map2k1 \\
\hline Nop56 & Srm \\
\hline Derl1 & Dnajb9 \\
\hline Wfs1 & Creld2 \\
\hline Prr16 & Gins2 \\
\hline Foxa2 & Srebf1 \\
\hline Nxpe2 & Aldh112 \\
\hline Sds & Psat1 \\
\hline Msh3 & Aldh18a1 \\
\hline Gadd45a & Ccne2 \\
\hline Nans & Cyb5r1 \\
\hline Gpr21 & Clstn1 \\
\hline Tmem67 & Nek4 \\
\hline Scara5 & Egfr \\
\hline Slc17a7 & Clstn3 \\
\hline Hsd3b5 & Scara5 \\
\hline Slc13a4 & Slc20a1 \\
\hline Suco & Sdf211 \\
\hline Esrp1 & ENSMUSG00000028536 \\
\hline Atf3 & Dnajb9 \\
\hline Acacb & Agpat9 \\
\hline Prlr & Acot3 \\
\hline Bhmt & Rgn \\
\hline Slc47a2 & Slc20a1 \\
\hline Tubb1 & Acat2 \\
\hline Abcg8 & Acat2 \\
\hline
\end{tabular}




\begin{tabular}{|c|c|}
\hline Pramef12 & Steap1 \\
\hline Trib3 & Slc7a11 \\
\hline Irx 3 & Foxa2 \\
\hline Spdya & Cdkn1a \\
\hline Acacb & Adck3 \\
\hline St6galnac4 & Pla2g4f \\
\hline Wars & $\mathrm{Mpdz}$ \\
\hline Wfs1 & Derl3 \\
\hline Trib3 & Psat1 \\
\hline Apoa5 & Bhmt \\
\hline Acpp & Fkbp11 \\
\hline Atf3 & Dnajc3 \\
\hline Mtnr1a & Nr1d1 \\
\hline Usp43 & Cep83 \\
\hline Rrs1 & Fkbp11 \\
\hline Apoa5 & Acss 2 \\
\hline Nupr1 & Gadd $45 b$ \\
\hline Creld2 & Dnajb9 \\
\hline St6galnac4 & B3gat2 \\
\hline Cox6a2 & Asb2 \\
\hline Hspa13 & P2ry2 \\
\hline Ddit3 & Derl1 \\
\hline Dcx & Ncam2 \\
\hline Jakmip1 & Slc17a8 \\
\hline
\end{tabular}

Table S2 WGCNA (Weighted correlation network analysis) network.

\begin{tabular}{ccc}
\hline fromNode & toNode & Weight \\
\hline Creld2 & Ddit3 & 0.70038 \\
& & 5 \\
Creld2 & Pdia4 & 0.85364 \\
& & 7 \\
Creld2 & & 0.81652 \\
& Syvn1 & 7 \\
\hline
\end{tabular}




\begin{tabular}{|c|c|c|}
\hline \multirow[t]{2}{*}{ Creld2 } & Srm & $\begin{array}{c}0.94581 \\
5\end{array}$ \\
\hline & & 0.82718 \\
\hline \multirow[t]{3}{*}{ Creld2 } & Hal & \\
\hline & & 2 \\
\hline & & 0.94069 \\
\hline \multirow[t]{2}{*}{ Creld2 } & Timd2 & \\
\hline & & 3 \\
\hline \multirow[t]{2}{*}{ Creld2 } & Nans & 0.95723 \\
\hline & & 0.82565 \\
\hline \multirow[t]{3}{*}{ Creld2 } & EG240327 & \\
\hline & & 6 \\
\hline & & 0.82405 \\
\hline \multirow[t]{3}{*}{ Creld2 } & 2810423A18Rik & \\
\hline & & 1 \\
\hline & & 0.81849 \\
\hline \multirow[t]{3}{*}{ Creld2 } & Derl3 & \\
\hline & & 6 \\
\hline & & 0.82331 \\
\hline \multirow[t]{3}{*}{ Creld2 } & Abcg5 & \\
\hline & & 6 \\
\hline & & 0.75238 \\
\hline \multirow[t]{2}{*}{ Creld2 } & Wfs 1 & \\
\hline & & 2 \\
\hline \multirow[t]{2}{*}{ Creld2 } & Psen2 & 0.78739 \\
\hline & & 0.74279 \\
\hline \multirow[t]{2}{*}{ Creld2 } & Klkb1 & \\
\hline & & 6 \\
\hline
\end{tabular}




\begin{tabular}{|c|c|c|}
\hline \multirow[t]{2}{*}{ Creld2 } & Nalp6 & $\begin{array}{c}0.81570 \\
6\end{array}$ \\
\hline & & 0.92454 \\
\hline \multirow[t]{3}{*}{ Creld2 } & Tlcd2 & \\
\hline & & 8 \\
\hline & & 0.71779 \\
\hline \multirow[t]{3}{*}{ Creld2 } & Aldh8a1 & \\
\hline & & 2 \\
\hline & & 0.81413 \\
\hline \multirow[t]{3}{*}{ Creld2 } & Acat2 & \\
\hline & & 1 \\
\hline & & 0.92390 \\
\hline \multirow[t]{3}{*}{ Creld2 } & Sucnr1 & \\
\hline & & 5 \\
\hline & & 0.78683 \\
\hline \multirow[t]{3}{*}{ Creld2 } & Lims2 & \\
\hline & & 1 \\
\hline & & 0.81305 \\
\hline \multirow[t]{3}{*}{ Creld2 } & Piga & \\
\hline & & 8 \\
\hline & & 0.90476 \\
\hline \multirow[t]{3}{*}{ Creld2 } & 1700019H03Rik & \\
\hline & & 8 \\
\hline & & 0.90856 \\
\hline \multirow[t]{3}{*}{ Creld2 } & BC048644 & \\
\hline & & 5 \\
\hline & & 0.79005 \\
\hline \multirow[t]{2}{*}{ Creld2 } & Abcg8 & \\
\hline & & 6 \\
\hline
\end{tabular}




\begin{tabular}{|c|c|c|}
\hline \multirow[t]{2}{*}{ Creld2 } & Proz & $\begin{array}{c}0.91307 \\
5\end{array}$ \\
\hline & & 0.79470 \\
\hline \multirow[t]{3}{*}{ Creld2 } & Apoa5 & \\
\hline & & 6 \\
\hline & & 0.87058 \\
\hline \multirow[t]{3}{*}{ Creld2 } & LOC240549 & \\
\hline & & 3 \\
\hline & & 0.70979 \\
\hline \multirow[t]{3}{*}{ Creld2 } & C730029A08Rik & \\
\hline & & 6 \\
\hline & & 0.92695 \\
\hline \multirow[t]{3}{*}{ Creld2 } & Ung & \\
\hline & & 5 \\
\hline & & 0.81085 \\
\hline \multirow[t]{3}{*}{ Creld2 } & Bdh1 & \\
\hline & & 4 \\
\hline & & 0.75934 \\
\hline \multirow[t]{2}{*}{ Creld2 } & C1qtnf1 & \\
\hline & & 9 \\
\hline \multirow[t]{2}{*}{ Creld2 } & Tmem25 & 0.85087 \\
\hline & & 0.84999 \\
\hline \multirow[t]{3}{*}{ Creld2 } & Cox6a2 & \\
\hline & & 5 \\
\hline & & 0.86648 \\
\hline \multirow[t]{3}{*}{ Creld2 } & Ccdc120 & \\
\hline & & 3 \\
\hline & & 0.82218 \\
\hline \multirow[t]{2}{*}{ Creld2 } & Dhcr7 & \\
\hline & & 5 \\
\hline
\end{tabular}




\begin{tabular}{|c|c|c|}
\hline Creld2 & Mist1 & 0.84414 \\
\hline & & 0.77513 \\
\hline \multirow[t]{3}{*}{ Creld2 } & Gpr146 & \\
\hline & & 5 \\
\hline & & 0.73367 \\
\hline \multirow[t]{3}{*}{ Creld2 } & Asns & \\
\hline & & 5 \\
\hline & & 0.73627 \\
\hline \multirow[t]{3}{*}{ Creld2 } & Abca8a & \\
\hline & & 3 \\
\hline & & 0.72736 \\
\hline \multirow[t]{3}{*}{ Creld2 } & Bhlhb8 & \\
\hline & & 5 \\
\hline & & 0.95627 \\
\hline \multirow[t]{2}{*}{ Creld2 } & Slc25a42 & \\
\hline & & 6 \\
\hline \multirow[t]{2}{*}{ Creld2 } & Pmm1 & 0.94238 \\
\hline & & 0.77157 \\
\hline \multirow[t]{3}{*}{ Creld2 } & Hsd3b5 & \\
\hline & & 3 \\
\hline & & 0.83908 \\
\hline \multirow[t]{3}{*}{ Creld2 } & Aox3 & \\
\hline & & 7 \\
\hline & & 0.93691 \\
\hline \multirow[t]{3}{*}{ Creld2 } & Smox & \\
\hline & & 4 \\
\hline & & 0.91862 \\
\hline \multirow[t]{2}{*}{ Creld2 } & LOC433886 & \\
\hline & & 9 \\
\hline
\end{tabular}




\begin{tabular}{|c|c|c|}
\hline \multirow[t]{2}{*}{ Creld2 } & Ngef & $\begin{array}{c}0.84913 \\
7\end{array}$ \\
\hline & & 0.72340 \\
\hline \multirow[t]{2}{*}{ Creld2 } & Zfp750 & \\
\hline & & 4 \\
\hline \multirow[t]{2}{*}{ Creld2 } & 1810020C02Rik & 0.86865 \\
\hline & & 0.86989 \\
\hline \multirow[t]{3}{*}{ Creld2 } & Slc41a3 & \\
\hline & & 4 \\
\hline & & 0.72885 \\
\hline \multirow[t]{3}{*}{ Creld2 } & Mcf2I & \\
\hline & & 7 \\
\hline & & 0.74749 \\
\hline \multirow[t]{3}{*}{ Creld2 } & 4432416J03Rik & \\
\hline & & 7 \\
\hline & & 0.79414 \\
\hline \multirow[t]{3}{*}{ Creld2 } & C430002P19Rik & \\
\hline & & 6 \\
\hline & & 0.75835 \\
\hline \multirow[t]{3}{*}{ Creld2 } & Ces1 & \\
\hline & & 2 \\
\hline & & 0.85970 \\
\hline \multirow[t]{3}{*}{ Creld2 } & D11Lgp2e & \\
\hline & & 9 \\
\hline & & 0.71358 \\
\hline \multirow[t]{3}{*}{ Creld2 } & Ube2u & \\
\hline & & 8 \\
\hline & & 0.96073 \\
\hline \multirow[t]{2}{*}{ Creld2 } & Bcdo2 & \\
\hline & & 9 \\
\hline
\end{tabular}




\begin{tabular}{|c|c|c|}
\hline \multirow[t]{2}{*}{ Creld2 } & Srd5a2 & $\begin{array}{c}0.71282 \\
2\end{array}$ \\
\hline & & 0.73321 \\
\hline \multirow[t]{3}{*}{ Creld2 } & Cnksr1 & \\
\hline & & 3 \\
\hline & & 0.73978 \\
\hline \multirow[t]{3}{*}{ Creld2 } & 5730410E15Rik & \\
\hline & & 2 \\
\hline & & 0.74783 \\
\hline \multirow[t]{3}{*}{ Creld2 } & E030049G20Rik & \\
\hline & & 5 \\
\hline & & 0.79325 \\
\hline \multirow[t]{3}{*}{ Creld2 } & Zfp800 & \\
\hline & & 7 \\
\hline & & 0.74777 \\
\hline \multirow[t]{2}{*}{ Creld2 } & Gpr152 & \\
\hline & & 4 \\
\hline \multirow[t]{2}{*}{ Ddit3 } & Fkbp11 & 0.70602 \\
\hline & & 0.72243 \\
\hline \multirow[t]{3}{*}{ Ddit3 } & Nupr1 & \\
\hline & & 7 \\
\hline & & 0.71493 \\
\hline \multirow[t]{3}{*}{ Ddit3 } & Timd2 & \\
\hline & & 5 \\
\hline & & 0.84376 \\
\hline \multirow[t]{3}{*}{ Ddit3 } & Nans & \\
\hline & & 3 \\
\hline & & 0.70843 \\
\hline \multirow[t]{2}{*}{ Ddit3 } & EG240327 & \\
\hline & & 7 \\
\hline
\end{tabular}




\begin{tabular}{|c|c|c|}
\hline \multirow[t]{2}{*}{ Ddit3 } & Cdkn1a & $\begin{array}{c}0.86462 \\
4\end{array}$ \\
\hline & & 0.90935 \\
\hline \multirow[t]{3}{*}{ Ddit3 } & 2810423A18Rik & \\
\hline & & 6 \\
\hline & & 0.74716 \\
\hline \multirow[t]{3}{*}{ Ddit3 } & Trib3 & \\
\hline & & 2 \\
\hline & & 0.75499 \\
\hline \multirow[t]{3}{*}{ Ddit3 } & Derl3 & \\
\hline & & 3 \\
\hline & & 0.70808 \\
\hline \multirow[t]{3}{*}{ Ddit3 } & Al848100 & \\
\hline & & 4 \\
\hline & & 0.70000 \\
\hline \multirow[t]{3}{*}{ Ddit3 } & Acat2 & \\
\hline & & 1 \\
\hline & & 0.94226 \\
\hline \multirow[t]{3}{*}{ Ddit3 } & Piga & \\
\hline & & 5 \\
\hline & & 0.84877 \\
\hline \multirow[t]{3}{*}{ Ddit3 } & 1700019H03Rik & \\
\hline & & 9 \\
\hline & & 0.90330 \\
\hline \multirow[t]{3}{*}{ Ddit3 } & Amigo3 & \\
\hline & & 7 \\
\hline & & 0.75847 \\
\hline \multirow[t]{2}{*}{ Ddit3 } & LOC240549 & \\
\hline & & 5 \\
\hline
\end{tabular}




\begin{tabular}{|c|c|c|}
\hline \multirow[t]{2}{*}{ Ddit3 } & Ung & $\begin{array}{c}0.78415 \\
3\end{array}$ \\
\hline & & 0.77089 \\
\hline \multirow[t]{3}{*}{ Ddit3 } & Mist1 & \\
\hline & & 2 \\
\hline & & 0.80652 \\
\hline \multirow[t]{3}{*}{ Ddit3 } & Bhlhb8 & \\
\hline & & 3 \\
\hline & & 0.74461 \\
\hline \multirow[t]{3}{*}{ Ddit3 } & Slc25a42 & \\
\hline & & 9 \\
\hline & & 0.86841 \\
\hline \multirow[t]{3}{*}{ Ddit3 } & LOC433886 & \\
\hline & & 2 \\
\hline & & 0.71329 \\
\hline \multirow[t]{3}{*}{ Ddit3 } & Ngef & \\
\hline & & 7 \\
\hline & & 0.89123 \\
\hline \multirow[t]{3}{*}{ Ddit3 } & Slc41a3 & \\
\hline & & 6 \\
\hline & & 0.83627 \\
\hline \multirow[t]{3}{*}{ Ddit3 } & Map3k6 & \\
\hline & & 6 \\
\hline & & 0.83242 \\
\hline \multirow[t]{3}{*}{ Ddit3 } & Gins2 & \\
\hline & & 1 \\
\hline & & 0.70641 \\
\hline \multirow[t]{2}{*}{ Ddit3 } & D11Lgp2e & \\
\hline & & 9 \\
\hline Ddit3 & 2610042014Rik & 0.74048 \\
\hline
\end{tabular}




\begin{tabular}{|c|c|c|}
\hline \multirow[t]{2}{*}{ Ddit3 } & B230207L18Rik & $\begin{array}{c}0.70904 \\
3\end{array}$ \\
\hline & & 0.84358 \\
\hline \multirow[t]{3}{*}{ Ddit3 } & Gpr152 & \\
\hline & & 1 \\
\hline & & 0.86255 \\
\hline \multirow[t]{3}{*}{ Fkbp11 } & Nupr1 & \\
\hline & & 1 \\
\hline & & 0.72880 \\
\hline \multirow[t]{3}{*}{ Fkbp11 } & Syvn1 & \\
\hline & & 3 \\
\hline & & 0.73724 \\
\hline \multirow[t]{2}{*}{ Fkbp11 } & Hal & \\
\hline & & 8 \\
\hline \multirow[t]{2}{*}{ Fkbp11 } & Dnajc3 & 0.73543 \\
\hline & & 0.75093 \\
\hline \multirow[t]{3}{*}{ Fkbp11 } & Timd2 & \\
\hline & & 8 \\
\hline & & 0.74155 \\
\hline \multirow[t]{3}{*}{ Fkbp11 } & Nans & \\
\hline & & 5 \\
\hline & & 0.81617 \\
\hline \multirow[t]{3}{*}{ Fkbp11 } & EG240327 & \\
\hline & & 5 \\
\hline & & 0.75026 \\
\hline \multirow[t]{3}{*}{ Fkbp11 } & Cdkn1a & \\
\hline & & 9 \\
\hline & & 0.77890 \\
\hline \multirow[t]{2}{*}{ Fkbp11 } & 2810423A18Rik & \\
\hline & & 5 \\
\hline
\end{tabular}




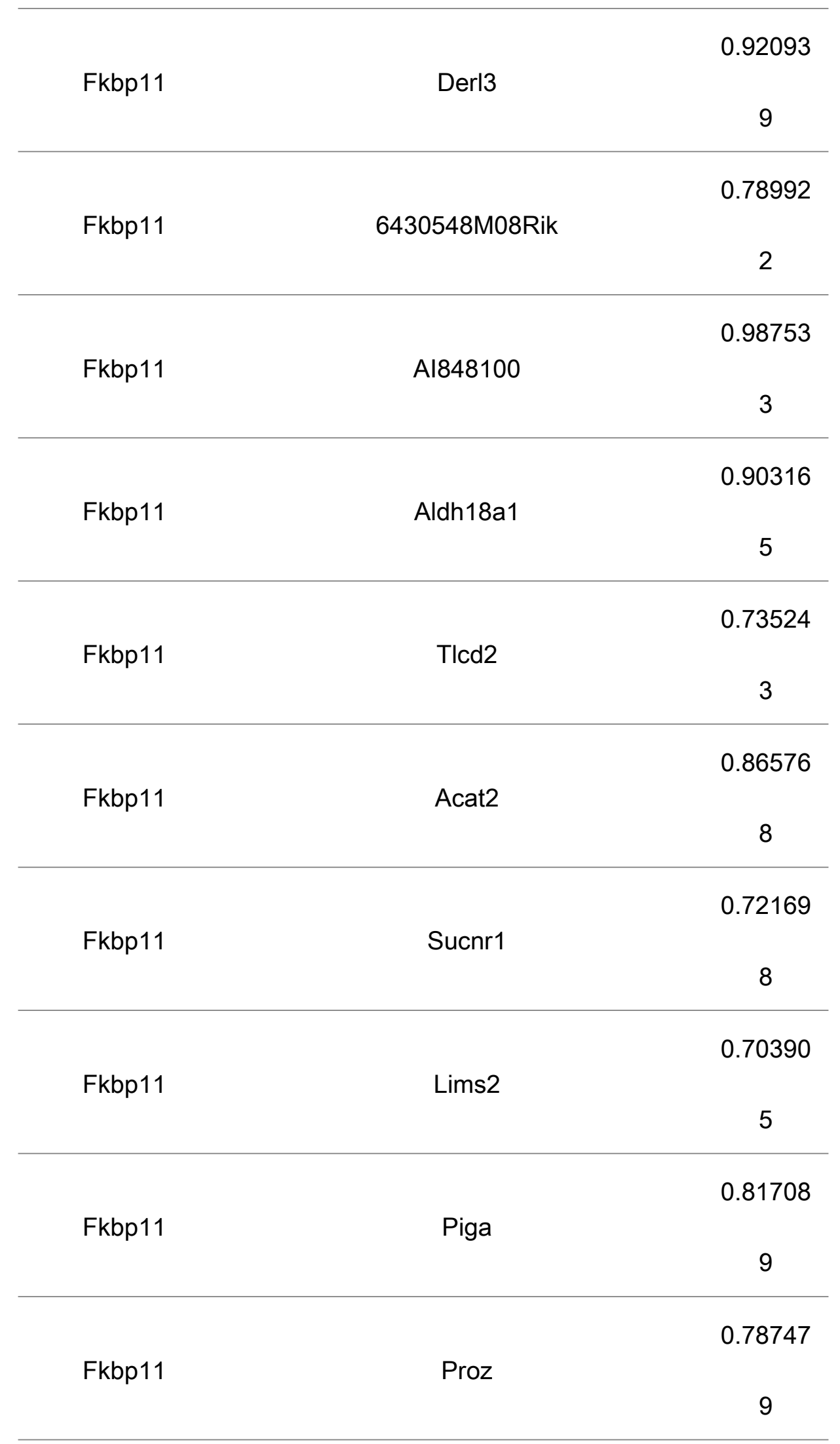




\begin{tabular}{|c|c|c|}
\hline Fkbp11 & LOC240549 & $\begin{array}{c}0.74079 \\
9\end{array}$ \\
\hline Fkbp11 & Ung & 0.82202 \\
\hline Fkbp11 & Ccdc120 & $\begin{array}{c}0.70533 \\
1\end{array}$ \\
\hline Fkbp11 & Dhcr7 & $\begin{array}{c}0.70497 \\
8\end{array}$ \\
\hline Fkbp11 & Gadd45a & $\begin{array}{c}0.80198 \\
6\end{array}$ \\
\hline Fkbp11 & Asns & $\begin{array}{c}0.90244 \\
4\end{array}$ \\
\hline Fkbp11 & Hpgd & $\begin{array}{c}0.91698 \\
1\end{array}$ \\
\hline Fkbp11 & Paqr7 & $\begin{array}{c}0.71799 \\
5\end{array}$ \\
\hline Fkbp11 & LOC433886 & $\begin{array}{c}0.70905 \\
1\end{array}$ \\
\hline Fkbp11 & Ngef & $\begin{array}{c}0.83928 \\
1\end{array}$ \\
\hline Fkbp11 & Slc41a3 & $\begin{array}{c}0.85322 \\
2\end{array}$ \\
\hline
\end{tabular}




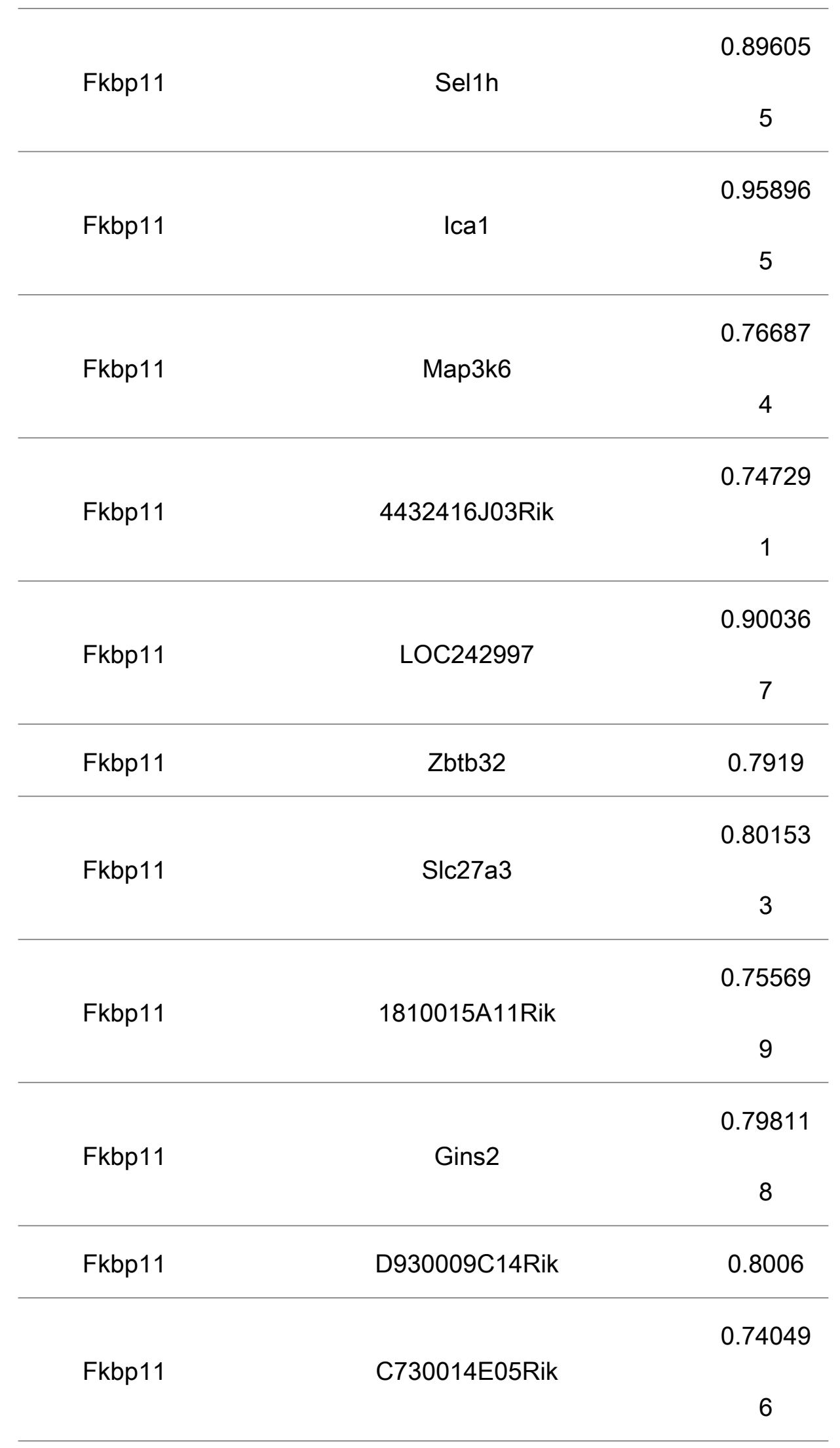




\begin{tabular}{|c|c|c|}
\hline Fkbp11 & Rock2 & $\begin{array}{c}0.75561 \\
1\end{array}$ \\
\hline & & 0.73893 \\
\hline \multirow{3}{*}{ Fkbp11 } & B230207L18Rik & \\
\hline & & 6 \\
\hline & & 0.70624 \\
\hline \multirow[t]{3}{*}{ Fkbp11 } & 1810046K07Rik & \\
\hline & & 7 \\
\hline & & 0.91844 \\
\hline \multirow[t]{3}{*}{ Nupr1 } & Ccdc134 & \\
\hline & & 6 \\
\hline & & 0.92261 \\
\hline \multirow[t]{3}{*}{ Nupr1 } & Cdkn1a & \\
\hline & & 2 \\
\hline & & 0.87203 \\
\hline \multirow[t]{3}{*}{ Nupr1 } & Trib3 & \\
\hline & & 3 \\
\hline & & 0.91072 \\
\hline \multirow[t]{3}{*}{ Nupr1 } & Al848100 & \\
\hline & & 9 \\
\hline & & 0.79585 \\
\hline \multirow[t]{3}{*}{ Nupr1 } & Aldh18a1 & \\
\hline & & 5 \\
\hline & & 0.71489 \\
\hline \multirow[t]{3}{*}{ Nupr1 } & Piga & \\
\hline & & 8 \\
\hline & & 0.83359 \\
\hline \multirow[t]{2}{*}{ Nupr1 } & Amigo3 & \\
\hline & & 1 \\
\hline
\end{tabular}




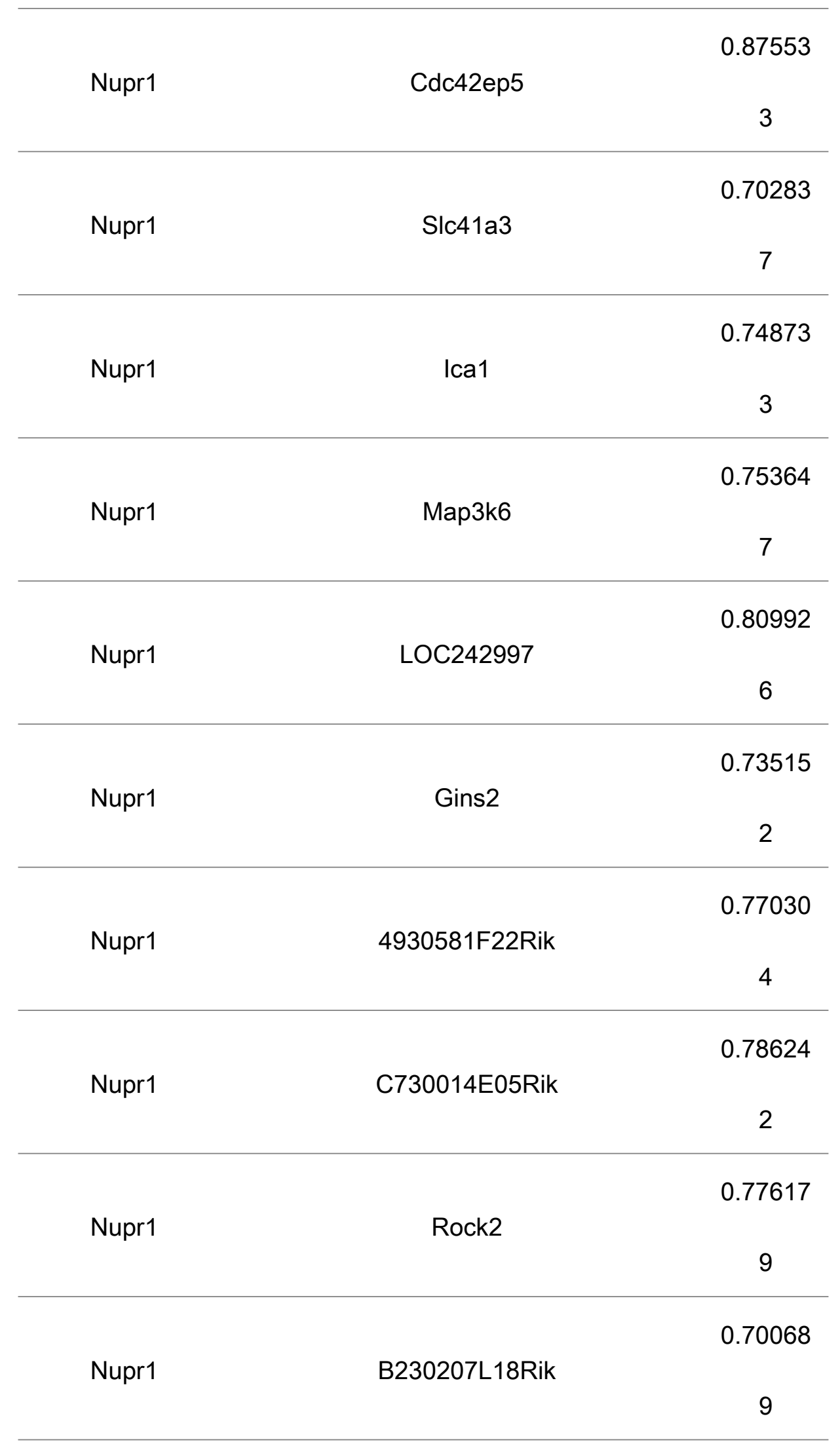




\begin{tabular}{|c|c|c|}
\hline \multirow[t]{2}{*}{ Pdia4 } & Syvn1 & $\begin{array}{c}0.97412 \\
8\end{array}$ \\
\hline & & 0.87288 \\
\hline \multirow[t]{3}{*}{ Pdia4 } & Srm & \\
\hline & & 1 \\
\hline & & 0.77285 \\
\hline \multirow[t]{2}{*}{ Pdia4 } & Hal & \\
\hline & & 5 \\
\hline \multirow[t]{2}{*}{ Pdia4 } & Dnajc3 & 0.84725 \\
\hline & & 0.83273 \\
\hline \multirow[t]{3}{*}{ Pdia4 } & Sdf2|1 & \\
\hline & & 9 \\
\hline & & 0.70803 \\
\hline \multirow[t]{3}{*}{ Pdia4 } & Tes & \\
\hline & & 6 \\
\hline & & 0.89094 \\
\hline \multirow[t]{2}{*}{ Pdia4 } & Timd2 & \\
\hline & & 7 \\
\hline \multirow[t]{2}{*}{ Pdia4 } & Nans & 0.79875 \\
\hline & & 0.85746 \\
\hline \multirow[t]{3}{*}{ Pdia4 } & EG240327 & \\
\hline & & 9 \\
\hline & & 0.82307 \\
\hline \multirow[t]{3}{*}{ Pdia4 } & Derl3 & \\
\hline & & 5 \\
\hline & & 0.75537 \\
\hline \multirow[t]{2}{*}{ Pdia4 } & 6430548M08Rik & \\
\hline & & 2 \\
\hline
\end{tabular}




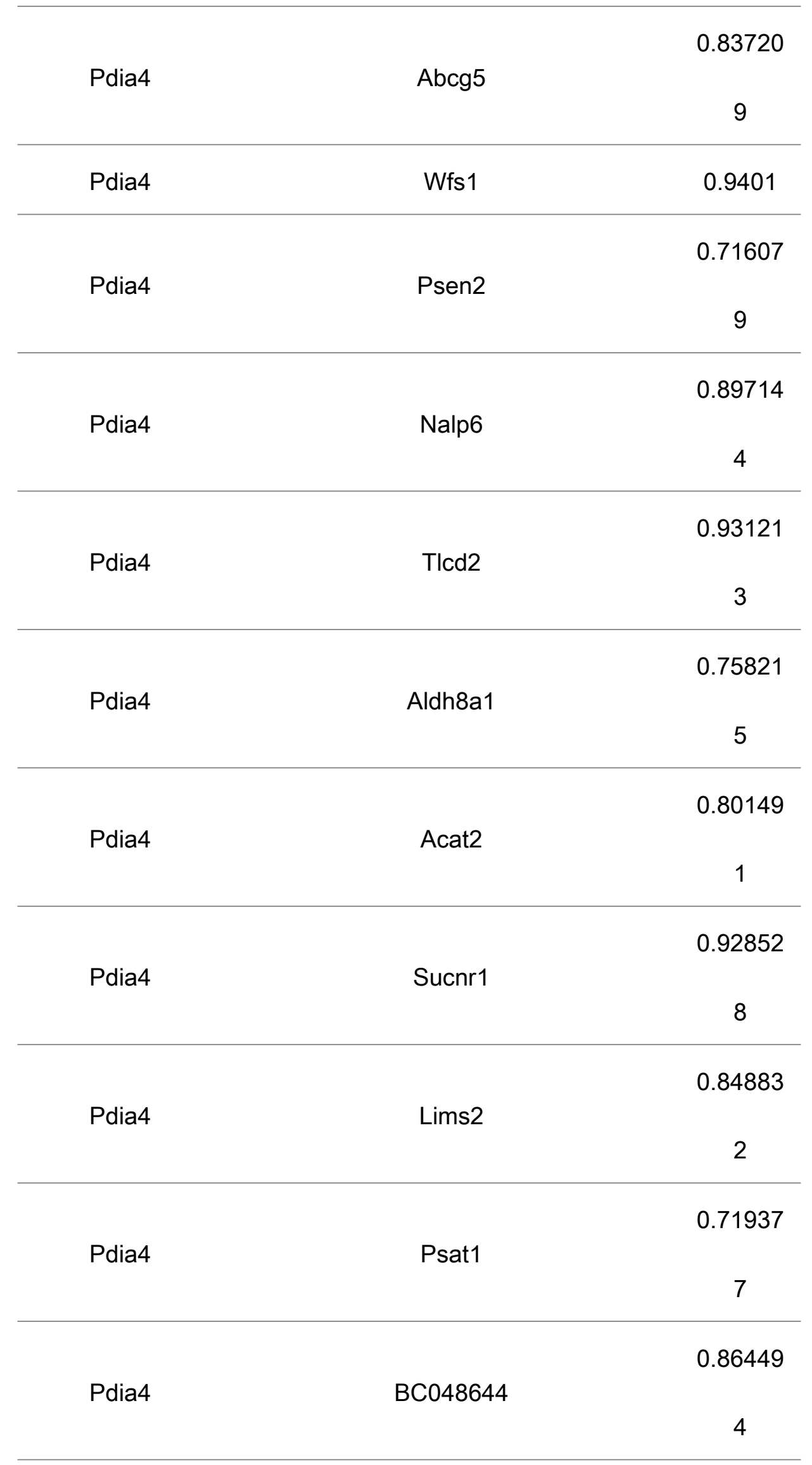




\begin{tabular}{|c|c|c|}
\hline \multirow[t]{2}{*}{ Pdia4 } & Abcg8 & $\begin{array}{c}0.70939 \\
3\end{array}$ \\
\hline & & 0.94074 \\
\hline \multirow[t]{3}{*}{ Pdia4 } & Proz & \\
\hline & & 9 \\
\hline & & 0.82956 \\
\hline \multirow[t]{2}{*}{ Pdia4 } & LOC240549 & \\
\hline & & 5 \\
\hline \multirow[t]{2}{*}{ Pdia4 } & Ung & 0.86612 \\
\hline & & 0.80274 \\
\hline \multirow[t]{2}{*}{ Pdia4 } & Bdh1 & \\
\hline & & 1 \\
\hline \multirow[t]{2}{*}{ Pdia4 } & Tmem25 & 0.8311 \\
\hline & & 0.73468 \\
\hline \multirow[t]{3}{*}{ Pdia4 } & Cox6a2 & \\
\hline & & 9 \\
\hline & & 0.98096 \\
\hline \multirow[t]{2}{*}{ Pdia4 } & Ccdc120 & \\
\hline & & 3 \\
\hline Pdia4 & Dhcr7 & 0.88352 \\
\hline \multirow[t]{2}{*}{ Pdia4 } & Gpr146 & 0.74429 \\
\hline & & 0.82809 \\
\hline \multirow[t]{3}{*}{ Pdia4 } & Asns & \\
\hline & & 8 \\
\hline & & 0.88156 \\
\hline \multirow[t]{2}{*}{ Pdia4 } & Abca8a & \\
\hline & & 3 \\
\hline
\end{tabular}




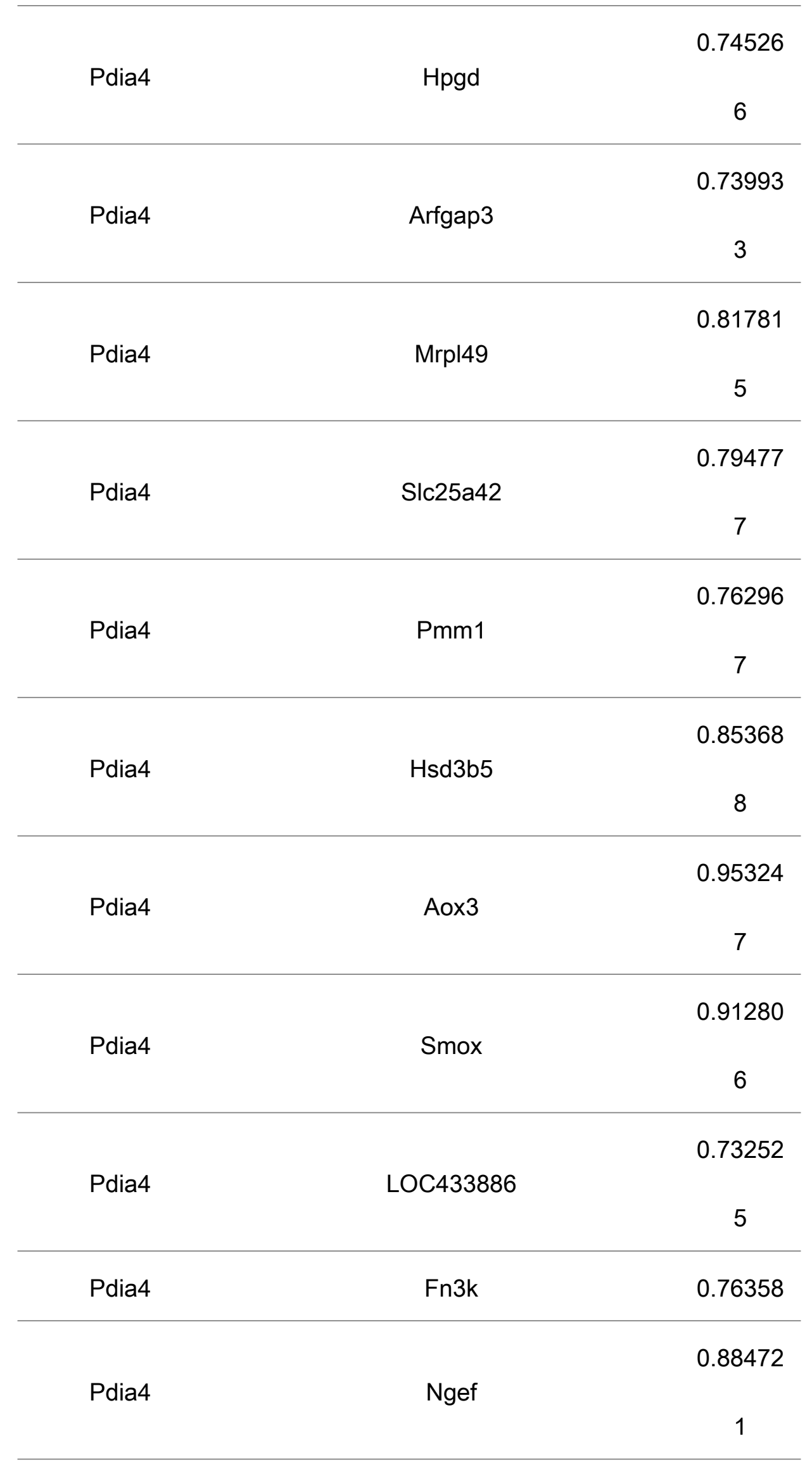




\begin{tabular}{|c|c|c|}
\hline Pdia4 & Zfp750 & $\begin{array}{c}0.85245 \\
7\end{array}$ \\
\hline Pdia4 & 1810020C02Rik & 0.77985 \\
\hline Pdia4 & Slc41a3 & 0.74552 \\
\hline Pdia4 & Sel1h & $\begin{array}{c}0.73093 \\
2\end{array}$ \\
\hline Pdia4 & Niban & $\begin{array}{c}0.70228 \\
7\end{array}$ \\
\hline Pdia4 & Fam129a & $\begin{array}{c}0.86135 \\
3\end{array}$ \\
\hline Pdia4 & Mcf2I & $\begin{array}{c}0.71151 \\
5\end{array}$ \\
\hline Pdia4 & Ces1 & $\begin{array}{c}0.79515 \\
6\end{array}$ \\
\hline Pdia4 & 4833403D03Rik & $\begin{array}{c}0.75570 \\
8\end{array}$ \\
\hline Pdia4 & Bves & 0.90165 \\
\hline Pdia4 & D11Lgp2e & $\begin{array}{c}0.73404 \\
8\end{array}$ \\
\hline Pdia4 & Bcdo2 & $\begin{array}{c}0.90652 \\
4\end{array}$ \\
\hline
\end{tabular}




\begin{tabular}{|c|c|c|}
\hline \multirow[t]{2}{*}{ Pdia4 } & 5730410E15Rik & $\begin{array}{c}0.95173 \\
8\end{array}$ \\
\hline & & 0.79870 \\
\hline \multirow[t]{2}{*}{ Syvn1 } & Srm & \\
\hline & & 5 \\
\hline \multirow[t]{2}{*}{ Syvn1 } & $\mathrm{Hal}$ & 0.78501 \\
\hline & & 0.92265 \\
\hline \multirow[t]{3}{*}{ Syvn1 } & Dnajc3 & \\
\hline & & 9 \\
\hline & & 0.85702 \\
\hline \multirow[t]{3}{*}{ Syvn1 } & Sdf2I1 & \\
\hline & & 2 \\
\hline & & 0.71311 \\
\hline \multirow[t]{3}{*}{ Syvn1 } & Tes & \\
\hline & & 2 \\
\hline & & 0.91538 \\
\hline \multirow[t]{3}{*}{ Syvn1 } & Timd2 & \\
\hline & & 2 \\
\hline & & 0.80419 \\
\hline \multirow[t]{3}{*}{ Syvn1 } & Nans & \\
\hline & & 1 \\
\hline & & 0.85789 \\
\hline \multirow[t]{3}{*}{ Syvn1 } & EG240327 & \\
\hline & & 1 \\
\hline & & 0.89101 \\
\hline \multirow[t]{3}{*}{ Syvn1 } & Derl3 & \\
\hline & & 8 \\
\hline & & 0.86673 \\
\hline \multirow[t]{2}{*}{ Syvn1 } & 6430548M08Rik & \\
\hline & & 5 \\
\hline
\end{tabular}




\begin{tabular}{|c|c|c|}
\hline Syvn1 & Abcg5 & $\begin{array}{c}0.78707 \\
9\end{array}$ \\
\hline & & 0.87898 \\
\hline \multirow[t]{2}{*}{ Syvn1 } & Wfs 1 & \\
\hline & & 1 \\
\hline Syvn1 & Psen2 & 0.739 \\
\hline Syvn1 & Klkb1 & 0.72202 \\
\hline \multirow[t]{2}{*}{ Syvn1 } & Nalp6 & 0.87066 \\
\hline & & 0.92742 \\
\hline \multirow[t]{3}{*}{ Syvn1 } & Tlcd2 & \\
\hline & & 2 \\
\hline & & 0.72128 \\
\hline \multirow[t]{3}{*}{ Syvn1 } & Aldh8a1 & \\
\hline & & 3 \\
\hline & & 0.87959 \\
\hline \multirow[t]{2}{*}{ Syvn1 } & Acat2 & \\
\hline & & 7 \\
\hline \multirow[t]{2}{*}{ Syvn1 } & Sucnr1 & 0.91231 \\
\hline & & 0.82437 \\
\hline \multirow[t]{3}{*}{ Syvn1 } & Lims2 & \\
\hline & & 6 \\
\hline & & 0.86266 \\
\hline \multirow[t]{3}{*}{ Syvn1 } & BC048644 & \\
\hline & & 7 \\
\hline & & 0.75757 \\
\hline \multirow[t]{2}{*}{ Syvn1 } & Abcg 8 & \\
\hline & & 3 \\
\hline
\end{tabular}




\begin{tabular}{|c|c|c|}
\hline \multirow[t]{2}{*}{ Syvn1 } & Proz & $\begin{array}{c}0.94549 \\
6\end{array}$ \\
\hline & & 0.81250 \\
\hline \multirow[t]{3}{*}{ Syvn1 } & LOC240549 & \\
\hline & & 9 \\
\hline & & 0.88877 \\
\hline \multirow[t]{3}{*}{ Syvn1 } & Ung & \\
\hline & & 2 \\
\hline & & 0.76626 \\
\hline \multirow[t]{3}{*}{ Syvn1 } & Bdh1 & \\
\hline & & 5 \\
\hline & & 0.77822 \\
\hline \multirow[t]{3}{*}{ Syvn1 } & Tmem25 & \\
\hline & & 3 \\
\hline & & 0.97710 \\
\hline \multirow[t]{3}{*}{ Syvn1 } & Ccdc120 & \\
\hline & & 2 \\
\hline & & 0.86643 \\
\hline \multirow[t]{3}{*}{ Syvn1 } & Dhcr7 & \\
\hline & & 9 \\
\hline & & 0.73936 \\
\hline \multirow[t]{3}{*}{ Syvn1 } & $\mathrm{Hr}$ & \\
\hline & & 2 \\
\hline & & 0.72911 \\
\hline \multirow[t]{3}{*}{ Syvn1 } & Gadd45a & \\
\hline & & 6 \\
\hline & & 0.76418 \\
\hline \multirow[t]{2}{*}{ Syvn1 } & Gpr146 & \\
\hline & & 6 \\
\hline Syvn1 & Asns & 0.89648 \\
\hline
\end{tabular}




\begin{tabular}{|c|c|c|}
\hline Syvn1 & Abca8a & $\begin{array}{c}0.83623 \\
2\end{array}$ \\
\hline & & 0.85839 \\
\hline \multirow[t]{2}{*}{ Syvn1 } & Hpgd & \\
\hline & & 4 \\
\hline \multirow[t]{2}{*}{ Syvn1 } & Mrpl49 & 0.78541 \\
\hline & & 0.78262 \\
\hline \multirow[t]{3}{*}{ Syvn1 } & Slc25a42 & \\
\hline & & 4 \\
\hline & & 0.72310 \\
\hline \multirow[t]{3}{*}{ Syvn1 } & Pmm1 & \\
\hline & & 5 \\
\hline & & 0.84272 \\
\hline \multirow[t]{3}{*}{ Syvn1 } & Hsd3b5 & \\
\hline & & 4 \\
\hline & & 0.89209 \\
\hline \multirow[t]{3}{*}{ Syvn1 } & Aox3 & \\
\hline & & 9 \\
\hline & & 0.84305 \\
\hline \multirow[t]{3}{*}{ Syvn1 } & Smox & \\
\hline & & 7 \\
\hline & & 0.71982 \\
\hline \multirow[t]{2}{*}{ Syvn1 } & LOC433886 & \\
\hline & & 9 \\
\hline \multirow[t]{2}{*}{ Syvn1 } & Fn3k & 0.72324 \\
\hline & & 0.89735 \\
\hline \multirow[t]{2}{*}{ Syvn1 } & Ngef & \\
\hline & & 3 \\
\hline
\end{tabular}




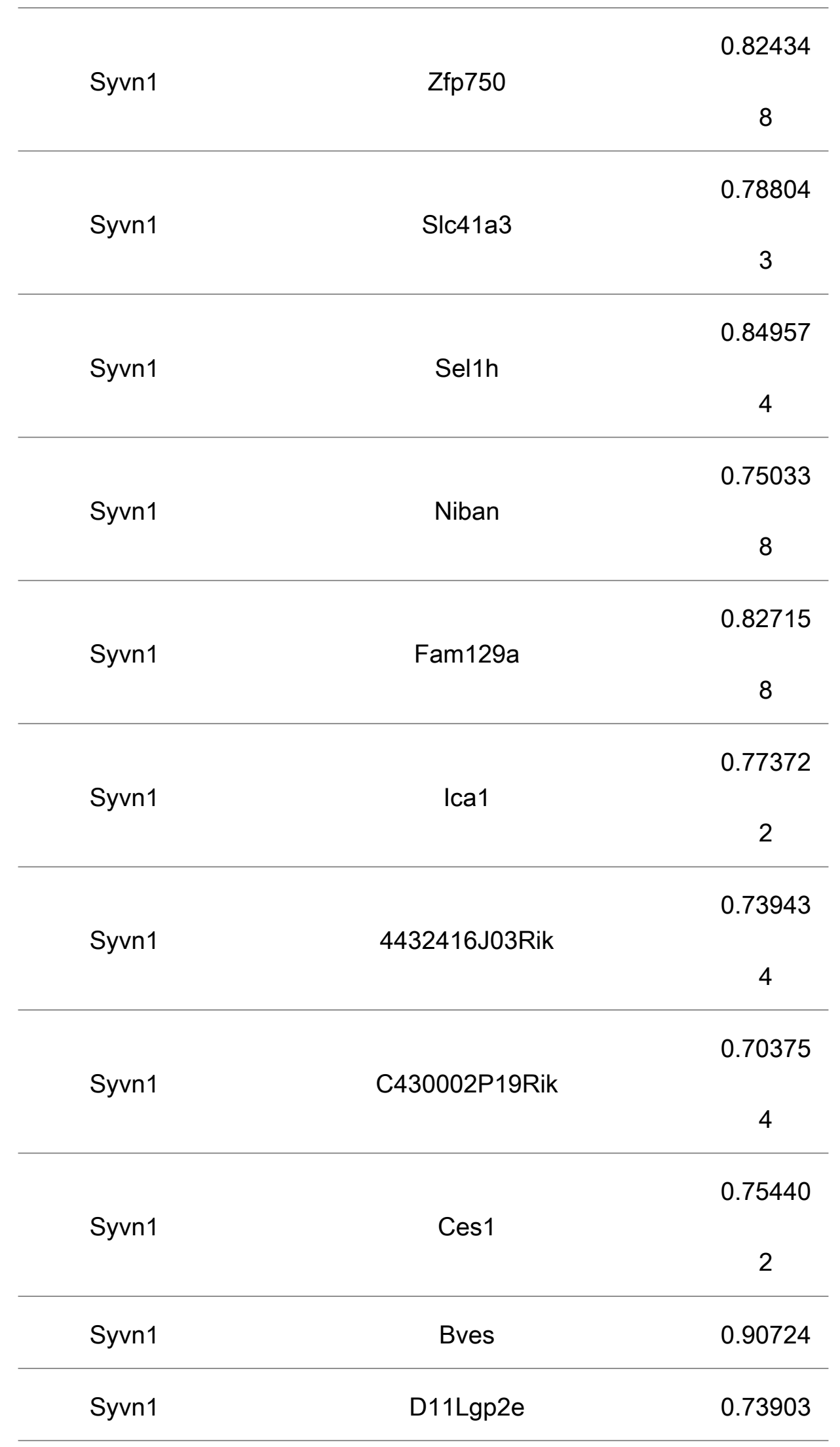




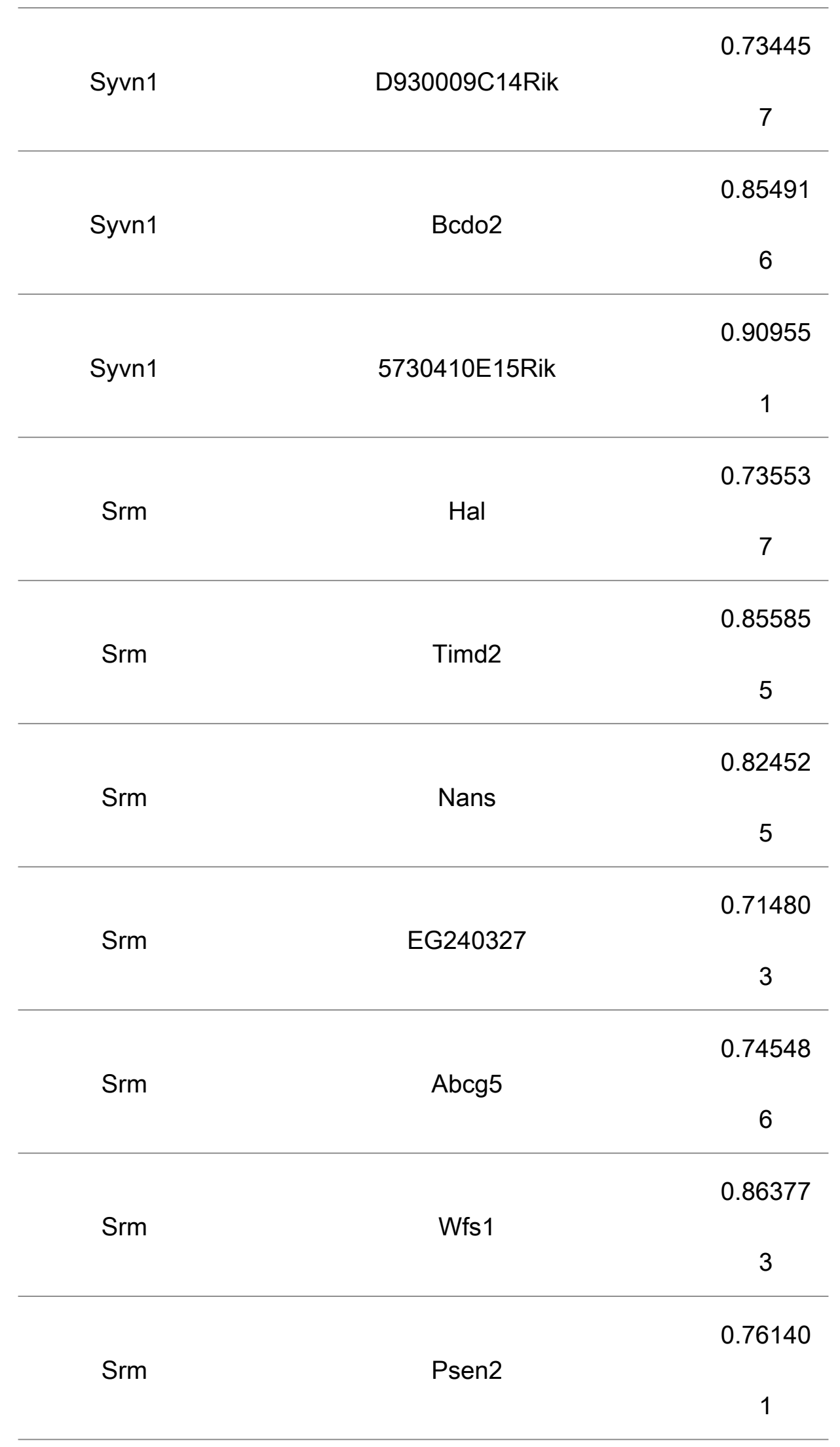




\begin{tabular}{|c|c|c|}
\hline \multirow[t]{2}{*}{ Srm } & Klkb1 & $\begin{array}{c}0.72556 \\
6\end{array}$ \\
\hline & & 0.75318 \\
\hline \multirow[t]{3}{*}{ Srm } & Nalp6 & \\
\hline & & 3 \\
\hline & & 0.85194 \\
\hline \multirow[t]{2}{*}{ Srm } & Tlcd2 & \\
\hline & & 5 \\
\hline \multirow[t]{2}{*}{ Srm } & Acat2 & 0.70033 \\
\hline & & 0.87050 \\
\hline \multirow[t]{3}{*}{ Srm } & Sucnr1 & \\
\hline & & 1 \\
\hline & & 0.73062 \\
\hline \multirow[t]{3}{*}{ Srm } & Lims2 & \\
\hline & & 2 \\
\hline & & 0.83960 \\
\hline \multirow[t]{3}{*}{ Srm } & Psat1 & \\
\hline & & 5 \\
\hline & & 0.76557 \\
\hline \multirow[t]{3}{*}{ Srm } & 1700019H03Rik & \\
\hline & & 8 \\
\hline & & 0.87127 \\
\hline \multirow[t]{3}{*}{ Srm } & Ptrh1 & \\
\hline & & 4 \\
\hline & & 0.83596 \\
\hline \multirow[t]{3}{*}{ Srm } & BC048644 & \\
\hline & & 1 \\
\hline & & 0.74305 \\
\hline \multirow[t]{2}{*}{ Srm } & Abcg8 & \\
\hline & & 7 \\
\hline
\end{tabular}




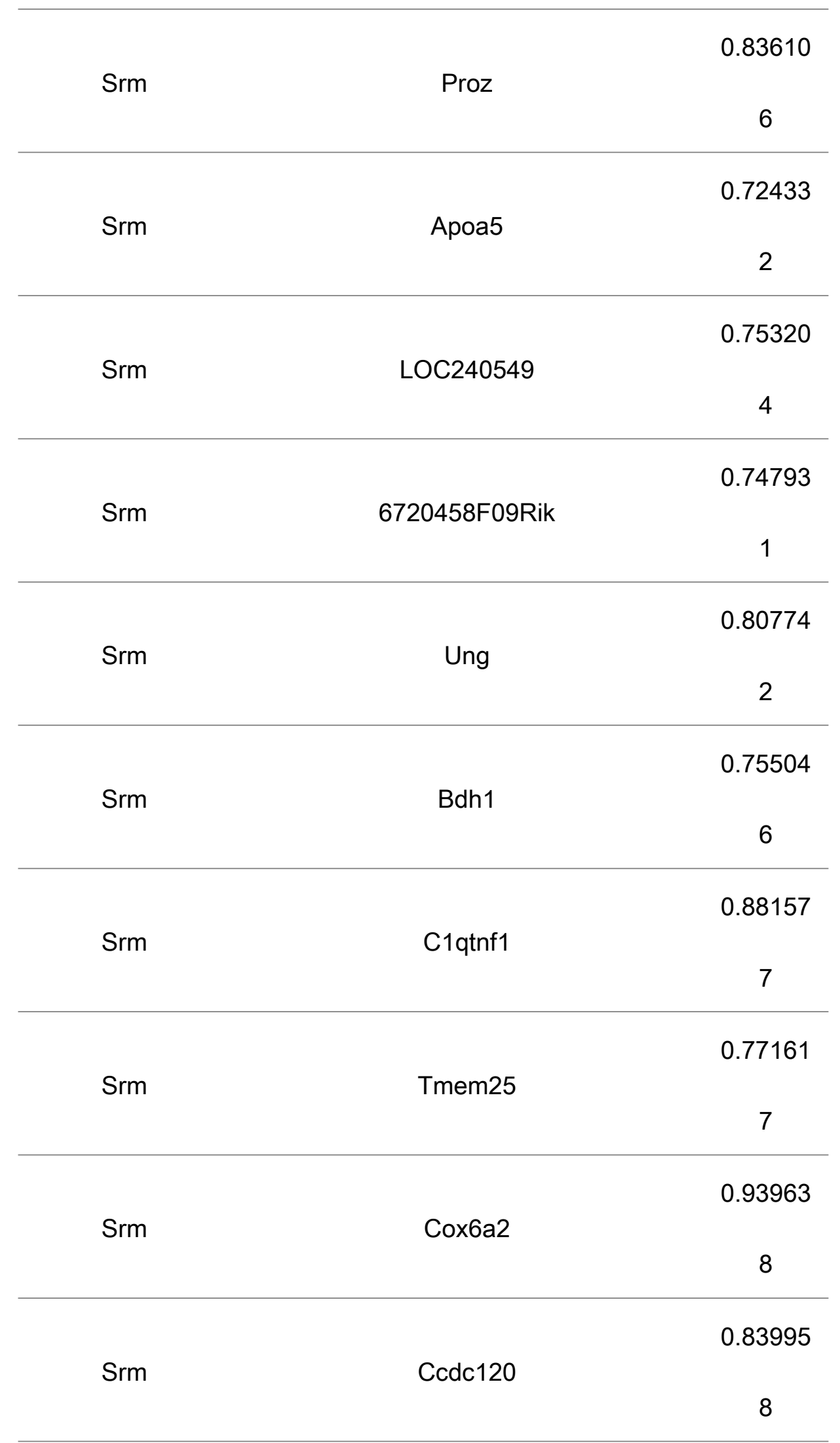




\begin{tabular}{|c|c|c|}
\hline \multirow[t]{2}{*}{ Srm } & Dhcr7 & $\begin{array}{c}0.77883 \\
7\end{array}$ \\
\hline & & 0.72771 \\
\hline \multirow[t]{3}{*}{ Srm } & Mist1 & \\
\hline & & 6 \\
\hline & & 0.70795 \\
\hline \multirow[t]{3}{*}{ Srm } & Gpr146 & \\
\hline & & 1 \\
\hline & & 0.72084 \\
\hline \multirow[t]{3}{*}{ Srm } & Abca8a & \\
\hline & & 7 \\
\hline & & 0.76233 \\
\hline \multirow[t]{3}{*}{ Srm } & Kdelr3 & \\
\hline & & 2 \\
\hline & & 0.86881 \\
\hline \multirow[t]{3}{*}{ Srm } & Slc25a42 & \\
\hline & & 6 \\
\hline & & 0.94949 \\
\hline \multirow[t]{2}{*}{ Srm } & Pmm1 & \\
\hline & & 1 \\
\hline \multirow[t]{2}{*}{ Srm } & Hsd3b5 & 0.70509 \\
\hline & & 0.85806 \\
\hline \multirow[t]{3}{*}{ Srm } & Aox3 & \\
\hline & & 4 \\
\hline & & 0.99450 \\
\hline \multirow[t]{3}{*}{ Srm } & Smox & \\
\hline & & 3 \\
\hline & & 0.77097 \\
\hline \multirow[t]{2}{*}{ Srm } & LOC433886 & \\
\hline & & 4 \\
\hline
\end{tabular}




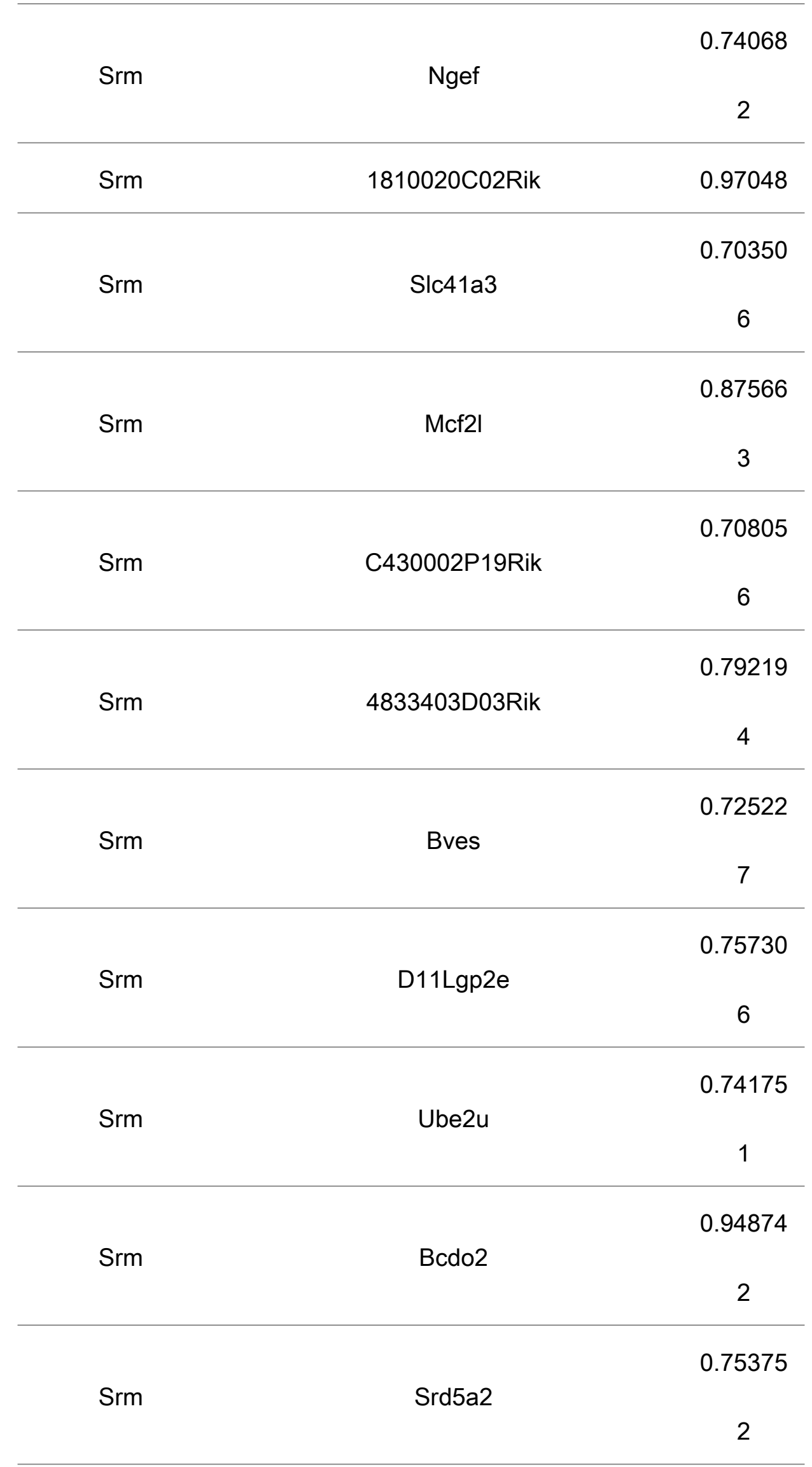




\begin{tabular}{|c|c|c|}
\hline \multirow[t]{2}{*}{ Srm } & 5730410E15Rik & $\begin{array}{c}0.80462 \\
1\end{array}$ \\
\hline & & 0.71295 \\
\hline \multirow[t]{3}{*}{ Srm } & EG666009 & \\
\hline & & 9 \\
\hline & & 0.87416 \\
\hline \multirow[t]{3}{*}{ Srm } & Zfp800 & \\
\hline & & 3 \\
\hline & & 0.83056 \\
\hline \multirow[t]{3}{*}{ Hal } & Timd2 & \\
\hline & & 4 \\
\hline & & 0.83936 \\
\hline \multirow[t]{2}{*}{ Hal } & Ddah1 & \\
\hline & & 2 \\
\hline \multirow[t]{2}{*}{$\mathrm{Hal}$} & Nans & 0.86631 \\
\hline & & 0.82120 \\
\hline \multirow[t]{3}{*}{$\mathrm{Hal}$} & EG240327 & \\
\hline & & 7 \\
\hline & & 0.74473 \\
\hline \multirow[t]{3}{*}{ Hal } & 2810423A18Rik & \\
\hline & & 4 \\
\hline & & 0.84646 \\
\hline \multirow[t]{3}{*}{ Hal } & Derl3 & \\
\hline & & 4 \\
\hline & & 0.84681 \\
\hline \multirow[t]{3}{*}{$\mathrm{Hal}$} & A530050D06Rik & \\
\hline & & 7 \\
\hline & & 0.76894 \\
\hline \multirow[t]{2}{*}{ Hal } & Abcg5 & \\
\hline & & 8 \\
\hline
\end{tabular}




\begin{tabular}{|c|c|c|}
\hline \multirow[t]{2}{*}{$\mathrm{Hal}$} & Psen2 & $\begin{array}{c}0.87912 \\
5\end{array}$ \\
\hline & & 0.86884 \\
\hline \multirow[t]{3}{*}{$\mathrm{Hal}$} & Klkb1 & \\
\hline & & 5 \\
\hline & & 0.78776 \\
\hline \multirow[t]{3}{*}{ Hal } & Tlcd2 & \\
\hline & & 9 \\
\hline & & 0.90281 \\
\hline \multirow[t]{3}{*}{ Hal } & Aldh8a1 & \\
\hline & & 4 \\
\hline & & 0.88890 \\
\hline \multirow[t]{3}{*}{ Hal } & Cldn2 & \\
\hline & & 1 \\
\hline & & 0.87346 \\
\hline \multirow[t]{2}{*}{$\mathrm{Hal}$} & Acat2 & \\
\hline & & 8 \\
\hline $\mathrm{Hal}$ & Sucnr1 & 0.93642 \\
\hline \multirow[t]{2}{*}{$\mathrm{Hal}$} & Lims2 & 0.91971 \\
\hline & & 0.76389 \\
\hline \multirow[t]{2}{*}{ Hal } & Piga & \\
\hline & & 2 \\
\hline \multirow[t]{2}{*}{ Hal } & 1110046J11Rik & 0.81974 \\
\hline & & 0.78038 \\
\hline \multirow[t]{3}{*}{$\mathrm{Hal}$} & 1700019H03Rik & \\
\hline & & 1 \\
\hline & & 0.81921 \\
\hline \multirow[t]{2}{*}{$\mathrm{Hal}$} & Abcg8 & \\
\hline & & 6 \\
\hline
\end{tabular}




\begin{tabular}{|c|c|c|}
\hline Hal & Proz & 0.87876 \\
\hline & & 0.97228 \\
\hline \multirow[t]{3}{*}{$\mathrm{Hal}$} & Apoa5 & \\
\hline & & 7 \\
\hline & & 0.75744 \\
\hline \multirow[t]{2}{*}{ Hal } & LOC240549 & \\
\hline & & 9 \\
\hline \multirow[t]{2}{*}{$\mathrm{Hal}$} & C730029A08Rik & 0.80689 \\
\hline & & 0.74276 \\
\hline \multirow[t]{3}{*}{ Hal } & LOC380998 & \\
\hline & & 2 \\
\hline & & 0.89965 \\
\hline \multirow[t]{3}{*}{ Hal } & Ung & \\
\hline & & 3 \\
\hline & & 0.94484 \\
\hline \multirow[t]{3}{*}{$\mathrm{Hal}$} & Bdh1 & \\
\hline & & 8 \\
\hline & & 0.81947 \\
\hline \multirow[t]{2}{*}{ Hal } & Tmem25 & \\
\hline & & 6 \\
\hline \multirow[t]{2}{*}{$\mathrm{Hal}$} & Ccdc120 & 0.76271 \\
\hline & & 0.94607 \\
\hline \multirow[t]{3}{*}{$\mathrm{Hal}$} & Dhcr7 & \\
\hline & & 8 \\
\hline & & 0.89417 \\
\hline \multirow[t]{3}{*}{$\mathrm{Hal}$} & Asns & \\
\hline & & 4 \\
\hline & & 0.81777 \\
\hline \multirow[t]{2}{*}{$\mathrm{Hal}$} & Abca8a & \\
\hline & & 1 \\
\hline
\end{tabular}




\begin{tabular}{|c|c|c|}
\hline $\mathrm{Hal}$ & 3010026009Rik & $\begin{array}{c}0.88013 \\
2\end{array}$ \\
\hline \multirow[t]{2}{*}{ Hal } & Paqr7 & 0.72311 \\
\hline & & 0.70936 \\
\hline \multirow[t]{3}{*}{ Hal } & Slc25a42 & \\
\hline & & 3 \\
\hline & & 0.71365 \\
\hline \multirow[t]{2}{*}{ Hal } & Pmm1 & \\
\hline & & 7 \\
\hline \multirow[t]{2}{*}{ Hal } & Aox3 & 0.82535 \\
\hline & & 0.73610 \\
\hline \multirow[t]{3}{*}{ Hal } & Smox & \\
\hline & & 4 \\
\hline & & 0.87176 \\
\hline \multirow[t]{3}{*}{$\mathrm{Hal}$} & LOC433886 & \\
\hline & & 4 \\
\hline & & 0.84075 \\
\hline \multirow[t]{3}{*}{ Hal } & Ngef & \\
\hline & & 4 \\
\hline & & 0.82742 \\
\hline \multirow[t]{3}{*}{ Hal } & Pdzk1ip1 & \\
\hline & & 4 \\
\hline & & 0.84062 \\
\hline \multirow[t]{3}{*}{ Hal } & Slc41a3 & \\
\hline & & 7 \\
\hline & & 0.84798 \\
\hline \multirow[t]{2}{*}{$\mathrm{Hal}$} & Ica1 & \\
\hline & & 8 \\
\hline Hal & Map3k6 & 0.78715 \\
\hline
\end{tabular}




\begin{tabular}{|c|c|c|}
\hline \multirow[t]{2}{*}{$\mathrm{Hal}$} & 4432416J03Rik & $\begin{array}{c}0.94458 \\
5\end{array}$ \\
\hline & & 0.87546 \\
\hline \multirow[t]{3}{*}{$\mathrm{Hal}$} & Slc27a3 & \\
\hline & & 3 \\
\hline & & 0.73438 \\
\hline \multirow[t]{3}{*}{$\mathrm{Hal}$} & C430002P19Rik & \\
\hline & & 4 \\
\hline & & 0.81852 \\
\hline \multirow[t]{3}{*}{ Hal } & Gins2 & \\
\hline & & 9 \\
\hline & & 0.98574 \\
\hline \multirow[t]{3}{*}{ Hal } & D11Lgp2e & \\
\hline & & 4 \\
\hline & & 0.75199 \\
\hline \multirow[t]{3}{*}{ Hal } & Zfp57 & \\
\hline & & 9 \\
\hline & & 0.88991 \\
\hline \multirow[t]{3}{*}{$\mathrm{Hal}$} & Bcdo2 & \\
\hline & & 6 \\
\hline & & 0.72523 \\
\hline \multirow[t]{3}{*}{ Hal } & Cnksr1 & \\
\hline & & 2 \\
\hline & & 0.84564 \\
\hline \multirow[t]{3}{*}{ Hal } & 2610042014Rik & \\
\hline & & 9 \\
\hline & & 0.74936 \\
\hline \multirow[t]{2}{*}{ Hal } & 5730410E15Rik & \\
\hline & & 5 \\
\hline
\end{tabular}




\begin{tabular}{|c|c|c|}
\hline \multirow[t]{2}{*}{$\mathrm{Hal}$} & B230207L18Rik & $\begin{array}{c}0.78056 \\
5\end{array}$ \\
\hline & & 0.87998 \\
\hline \multirow[t]{3}{*}{ Dnajc3 } & Sdf2I1 & \\
\hline & & 3 \\
\hline & & 0.77269 \\
\hline \multirow[t]{3}{*}{ Dnajc3 } & Tes & \\
\hline & & 6 \\
\hline & & 0.74603 \\
\hline \multirow[t]{3}{*}{ Dnajc3 } & Timd2 & \\
\hline & & 8 \\
\hline & & 0.74484 \\
\hline \multirow[t]{3}{*}{ Dnajc3 } & EG240327 & \\
\hline & & 3 \\
\hline & & 0.80463 \\
\hline \multirow[t]{3}{*}{ Dnajc3 } & Derl3 & \\
\hline & & 7 \\
\hline & & 0.97065 \\
\hline \multirow[t]{3}{*}{ Dnajc3 } & 6430548M08Rik & \\
\hline & & 6 \\
\hline & & 0.74627 \\
\hline \multirow[t]{3}{*}{ Dnajc3 } & Wfs1 & \\
\hline & & 3 \\
\hline & & 0.71087 \\
\hline \multirow[t]{3}{*}{ Dnajc3 } & Aldh18a1 & \\
\hline & & 3 \\
\hline & & 0.74511 \\
\hline \multirow[t]{2}{*}{ Dnajc3 } & Nalp6 & \\
\hline & & 5 \\
\hline
\end{tabular}




\begin{tabular}{|c|c|c|}
\hline \multirow[t]{2}{*}{ Dnajc3 } & Tlcd2 & $\begin{array}{c}0.76512 \\
2\end{array}$ \\
\hline & & 0.78700 \\
\hline \multirow[t]{3}{*}{ Dnajc3 } & Acat2 & \\
\hline & & 4 \\
\hline & & 0.75125 \\
\hline \multirow[t]{3}{*}{ Dnajc3 } & Sucnr1 & \\
\hline & & 7 \\
\hline & & 0.71448 \\
\hline \multirow[t]{3}{*}{ Dnajc3 } & Lims2 & \\
\hline & & 5 \\
\hline & & 0.80411 \\
\hline \multirow[t]{3}{*}{ Dnajc3 } & Proz & \\
\hline & & 2 \\
\hline & & 0.73230 \\
\hline \multirow[t]{3}{*}{ Dnajc3 } & Ung & \\
\hline & & 5 \\
\hline & & 0.85457 \\
\hline \multirow[t]{3}{*}{ Dnajc3 } & Ccdc120 & \\
\hline & & 2 \\
\hline & & 0.74827 \\
\hline \multirow[t]{3}{*}{ Dnajc3 } & Dhcr7 & \\
\hline & & 4 \\
\hline & & 0.84685 \\
\hline \multirow[t]{3}{*}{ Dnajc3 } & $\mathrm{Hr}$ & \\
\hline & & 6 \\
\hline & & 0.86923 \\
\hline \multirow[t]{2}{*}{ Dnajc3 } & Gadd45a & \\
\hline & & 9 \\
\hline
\end{tabular}




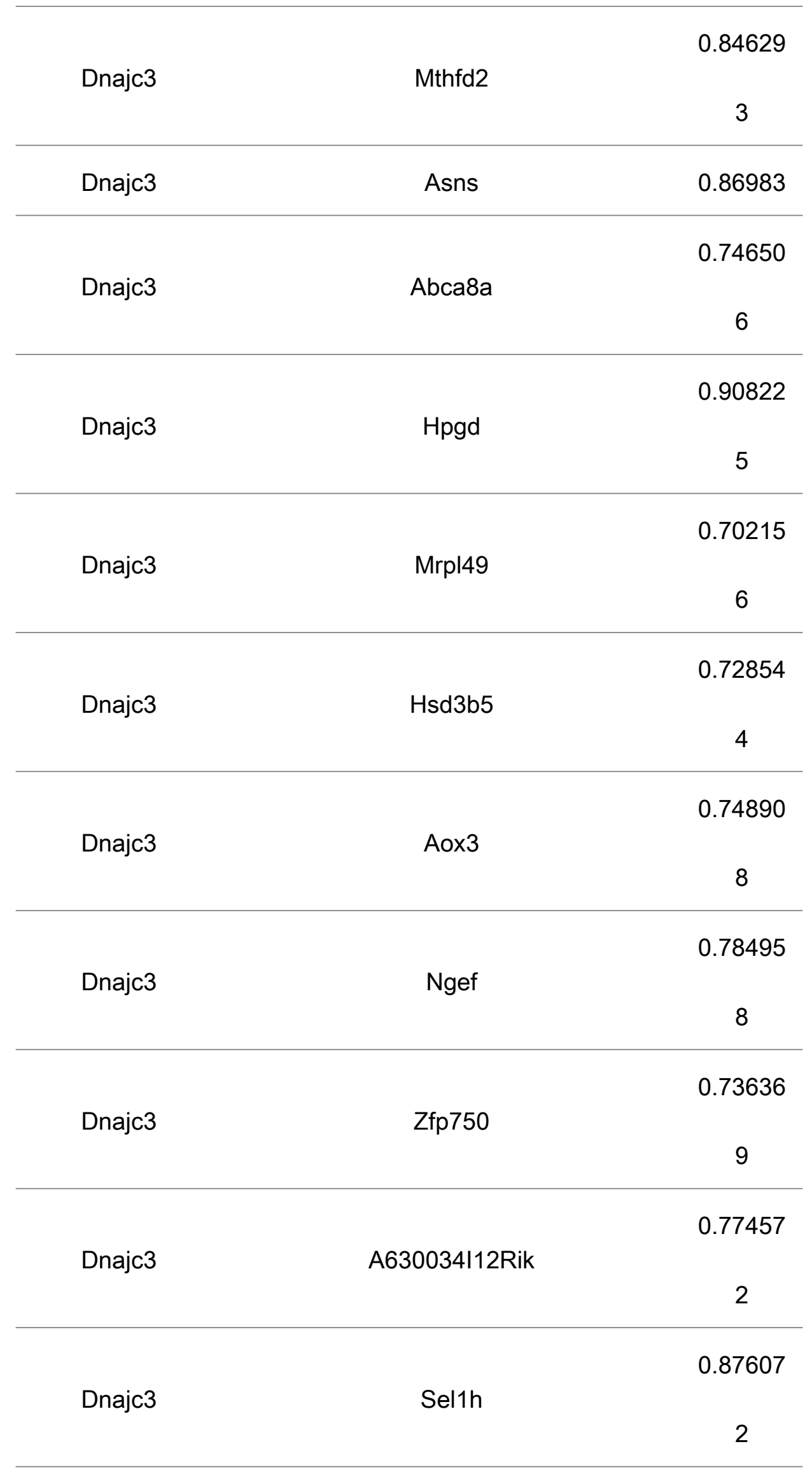




\begin{tabular}{|c|c|c|}
\hline \multirow[t]{2}{*}{ Dnajc3 } & Niban & $\begin{array}{c}0.90567 \\
8\end{array}$ \\
\hline & & 0.75867 \\
\hline \multirow[t]{3}{*}{ Dnajc3 } & Fam129a & \\
\hline & & 2 \\
\hline & & 0.77531 \\
\hline \multirow[t]{3}{*}{ Dnajc3 } & Ica1 & \\
\hline & & 9 \\
\hline & & 0.79220 \\
\hline \multirow[t]{3}{*}{ Dnajc3 } & 1810015A11Rik & \\
\hline & & 5 \\
\hline & & 0.86138 \\
\hline \multirow[t]{3}{*}{ Dnajc3 } & Bves & \\
\hline & & 7 \\
\hline & & 0.84785 \\
\hline \multirow[t]{3}{*}{ Dnajc3 } & D930009C14Rik & \\
\hline & & 3 \\
\hline & & 0.82029 \\
\hline \multirow[t]{3}{*}{ Dnajc3 } & 5730410E15Rik & \\
\hline & & 6 \\
\hline & & 0.80006 \\
\hline \multirow[t]{3}{*}{ Dnajc3 } & 1810008K04Rik & \\
\hline & & 4 \\
\hline & & 0.82567 \\
\hline \multirow[t]{3}{*}{ Dnajc3 } & 5830411E10Rik & \\
\hline & & 9 \\
\hline & & 0.76110 \\
\hline \multirow[t]{2}{*}{ Dnajc3 } & Ranbp3I & \\
\hline & & 3 \\
\hline Sdf2I1 & Tes & 0.95327 \\
\hline
\end{tabular}




\begin{tabular}{|c|c|c|}
\hline \multirow[t]{2}{*}{ Sdf2I1 } & 6430548M08Rik & $\begin{array}{c}0.79895 \\
1\end{array}$ \\
\hline & & 0.83064 \\
\hline \multirow[t]{2}{*}{ Sdf2I1 } & Pycr2 & \\
\hline & & 5 \\
\hline \multirow[t]{2}{*}{ Sdf2I1 } & Wfs 1 & 0.87107 \\
\hline & & 0.73573 \\
\hline \multirow[t]{3}{*}{ Sdf2I1 } & Gmds & \\
\hline & & 6 \\
\hline & & 0.77093 \\
\hline \multirow[t]{3}{*}{ Sdf2I1 } & Ccdc120 & \\
\hline & & 7 \\
\hline & & 0.95985 \\
\hline \multirow[t]{3}{*}{ Sdf2I1 } & $\mathrm{Hr}$ & \\
\hline & & 1 \\
\hline & & 0.87058 \\
\hline \multirow[t]{3}{*}{ Sdf2I1 } & Mthfd2 & \\
\hline & & 4 \\
\hline & & 0.88456 \\
\hline \multirow[t]{3}{*}{ Sdf2I1 } & Arfgap3 & \\
\hline & & 3 \\
\hline & & 0.73826 \\
\hline \multirow[t]{3}{*}{ Sdf2I1 } & Aox3 & \\
\hline & & 1 \\
\hline & & 0.78613 \\
\hline \multirow[t]{3}{*}{ Sdf2I1 } & A630034I12Rik & \\
\hline & & 4 \\
\hline & & 0.86779 \\
\hline \multirow[t]{2}{*}{ Sdf2I1 } & Niban & \\
\hline & & 6 \\
\hline
\end{tabular}




\begin{tabular}{|c|c|c|}
\hline \multirow[t]{2}{*}{ Sdf2I1 } & Bves & $\begin{array}{c}0.93631 \\
7\end{array}$ \\
\hline & & 0.88464 \\
\hline \multirow[t]{3}{*}{ Sdf2I1 } & 5730410E15Rik & \\
\hline & & 3 \\
\hline & & 0.82358 \\
\hline \multirow[t]{3}{*}{ Sdf2I1 } & 5830411E10Rik & \\
\hline & & 8 \\
\hline & & 0.91335 \\
\hline \multirow[t]{3}{*}{ Tes } & Pycr2 & \\
\hline & & 3 \\
\hline & & 0.79859 \\
\hline \multirow[t]{3}{*}{ Tes } & Wfs 1 & \\
\hline & & 2 \\
\hline & & 0.77404 \\
\hline \multirow[t]{3}{*}{ Tes } & Gmds & \\
\hline & & 5 \\
\hline & & 0.96890 \\
\hline \multirow[t]{3}{*}{ Tes } & $\mathrm{Hr}$ & \\
\hline & & 2 \\
\hline & & 0.85211 \\
\hline \multirow[t]{3}{*}{ Tes } & Mthfd2 & \\
\hline & & 1 \\
\hline & & 0.94711 \\
\hline \multirow[t]{3}{*}{ Tes } & Arfgap3 & \\
\hline & & 7 \\
\hline & & 0.74853 \\
\hline \multirow[t]{2}{*}{ Tes } & A630034I12Rik & \\
\hline & & 8 \\
\hline
\end{tabular}




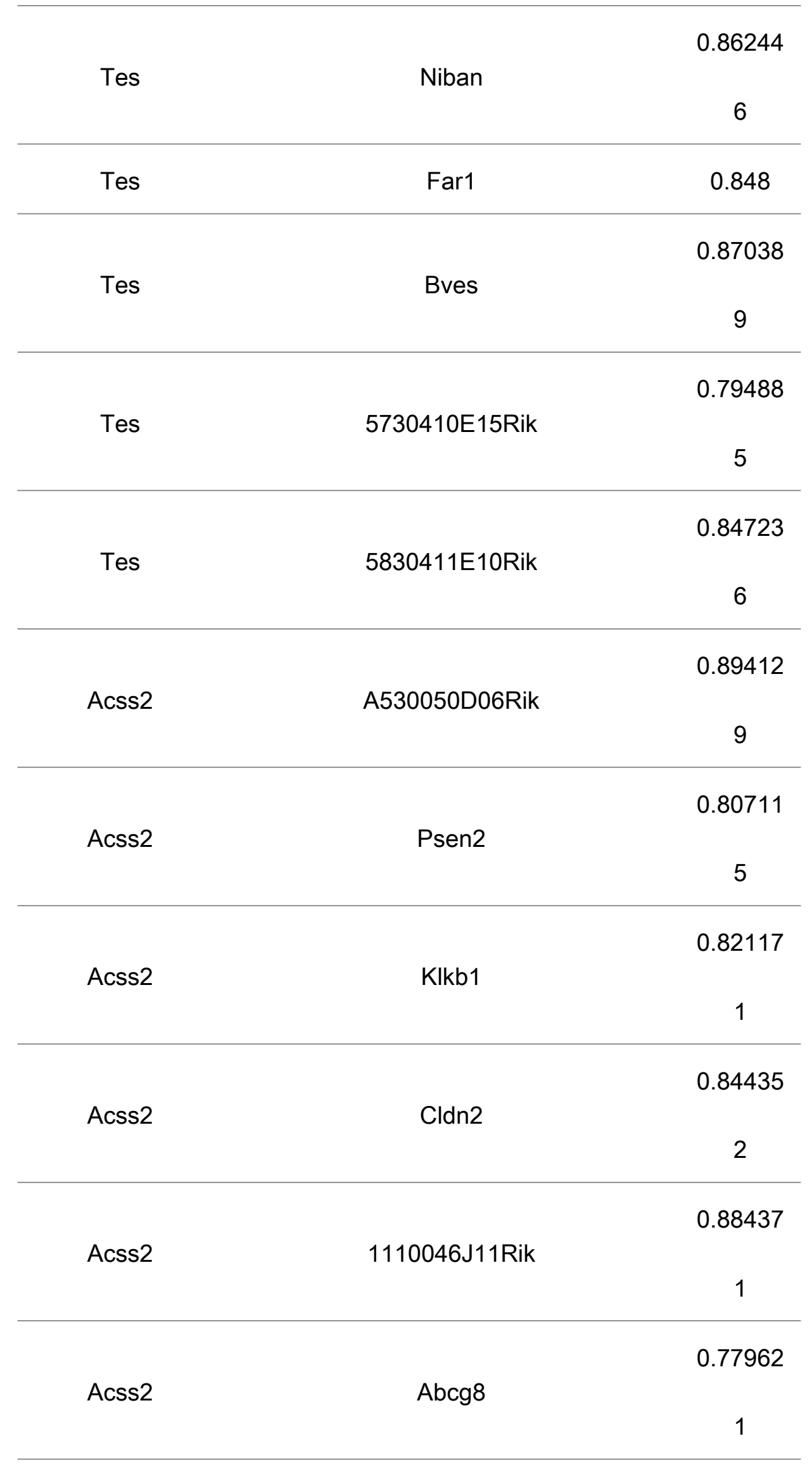




\begin{tabular}{|c|c|c|}
\hline \multirow[t]{2}{*}{ Acss2 } & Raet1b & $\begin{array}{c}0.81160 \\
4\end{array}$ \\
\hline & & 0.87396 \\
\hline \multirow[t]{3}{*}{ Acss2 } & C730029A08Rik & \\
\hline & & 9 \\
\hline & & 0.70761 \\
\hline \multirow[t]{3}{*}{ Acss2 } & Acacb & \\
\hline & & 8 \\
\hline & & 0.83885 \\
\hline \multirow[t]{3}{*}{ Acss2 } & Pdzk1ip1 & \\
\hline & & 5 \\
\hline & & 0.80154 \\
\hline \multirow[t]{3}{*}{ Acss2 } & A530020H22Rik & \\
\hline & & 3 \\
\hline & & 0.73734 \\
\hline \multirow[t]{3}{*}{ Acss2 } & 4432416J03Rik & \\
\hline & & 2 \\
\hline & & 0.79015 \\
\hline \multirow[t]{3}{*}{ Acss2 } & 2610528J11Rik & \\
\hline & & 5 \\
\hline & & 0.79112 \\
\hline \multirow[t]{3}{*}{ Acss2 } & Ube2u & \\
\hline & & 8 \\
\hline & & 0.81022 \\
\hline \multirow[t]{3}{*}{ Acss2 } & Cnksr1 & \\
\hline & & 8 \\
\hline & & 0.72314 \\
\hline \multirow[t]{2}{*}{ Igfals } & Selenbp2 & \\
\hline & & 6 \\
\hline Igfals & Slc25a23 & 0.78083 \\
\hline
\end{tabular}




\begin{tabular}{|c|c|c|}
\hline Igfals & $\mathrm{Nfe} 2$ & 0.85545 \\
\hline & & 0.77695 \\
\hline \multirow[t]{3}{*}{ Igfals } & D430030K24Rik & \\
\hline & & 7 \\
\hline & & 0.95554 \\
\hline \multirow[t]{3}{*}{ Timd2 } & Nans & \\
\hline & & 3 \\
\hline & & 0.86665 \\
\hline \multirow[t]{3}{*}{ Timd2 } & EG240327 & \\
\hline & & 9 \\
\hline & & 0.83982 \\
\hline \multirow[t]{3}{*}{ Timd2 } & 2810423A18Rik & \\
\hline & & 3 \\
\hline & & 0.93996 \\
\hline \multirow[t]{3}{*}{ Timd2 } & Derl3 & \\
\hline & & 6 \\
\hline & & 0.72726 \\
\hline \multirow[t]{3}{*}{ Timd2 } & 6430548M08Rik & \\
\hline & & 8 \\
\hline & & 0.70460 \\
\hline \multirow[t]{3}{*}{ Timd2 } & Al848100 & \\
\hline & & 6 \\
\hline & & 0.79408 \\
\hline \multirow[t]{3}{*}{ Timd2 } & Abcg5 & \\
\hline & & 1 \\
\hline & & 0.75589 \\
\hline \multirow[t]{3}{*}{ Timd2 } & Wfs 1 & \\
\hline & & 5 \\
\hline & & 0.79884 \\
\hline \multirow[t]{2}{*}{ Timd2 } & Psen2 & \\
\hline & & 8 \\
\hline
\end{tabular}




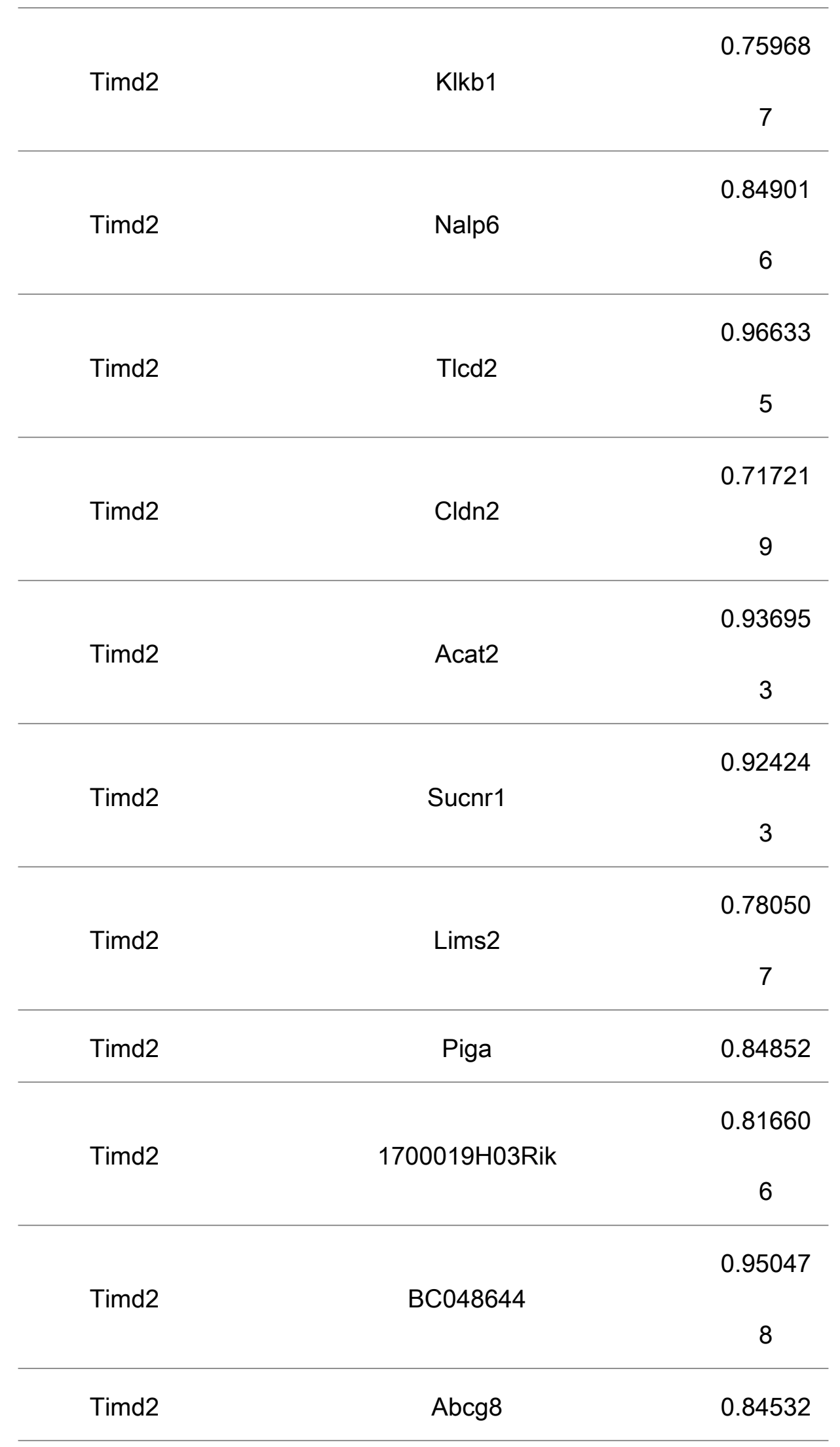




\begin{tabular}{|c|c|c|}
\hline \multirow[t]{2}{*}{ Timd2 } & Proz & $\begin{array}{c}0.95819 \\
5\end{array}$ \\
\hline & & 0.74075 \\
\hline \multirow[t]{3}{*}{ Timd2 } & Apoa5 & \\
\hline & & 3 \\
\hline & & 0.87998 \\
\hline \multirow[t]{3}{*}{ Timd2 } & LOC240549 & \\
\hline & & 2 \\
\hline & & 0.97324 \\
\hline \multirow[t]{3}{*}{ Timd2 } & Ung & \\
\hline & & 9 \\
\hline & & 0.76500 \\
\hline \multirow[t]{3}{*}{ Timd2 } & Bdh1 & \\
\hline & & 7 \\
\hline & & 0.80243 \\
\hline \multirow[t]{2}{*}{ Timd2 } & Tmem25 & \\
\hline & & 6 \\
\hline \multirow[t]{2}{*}{ Timd2 } & Ccdc120 & 0.93048 \\
\hline & & 0.82416 \\
\hline \multirow[t]{3}{*}{ Timd2 } & Dhcr7 & \\
\hline & & 6 \\
\hline & & 0.71300 \\
\hline \multirow[t]{3}{*}{ Timd2 } & Mist1 & \\
\hline & & 5 \\
\hline & & 0.85806 \\
\hline \multirow[t]{3}{*}{ Timd2 } & Gpr146 & \\
\hline & & 5 \\
\hline & & 0.85211 \\
\hline \multirow[t]{2}{*}{ Timd2 } & Asns & \\
\hline & & 4 \\
\hline
\end{tabular}




\begin{tabular}{|c|c|c|}
\hline \multirow[t]{2}{*}{ Timd2 } & Abca8a & $\begin{array}{c}0.73265 \\
2\end{array}$ \\
\hline & & 0.79935 \\
\hline \multirow[t]{3}{*}{ Timd2 } & Hpgd & \\
\hline & & 2 \\
\hline & & 0.72551 \\
\hline \multirow[t]{3}{*}{ Timd2 } & Mrpl49 & \\
\hline & & 6 \\
\hline & & 0.94404 \\
\hline \multirow[t]{3}{*}{ Timd2 } & Slc25a42 & \\
\hline & & 6 \\
\hline & & 0.86353 \\
\hline \multirow[t]{3}{*}{ Timd2 } & Pmm1 & \\
\hline & & 2 \\
\hline & & 0.83236 \\
\hline \multirow[t]{3}{*}{ Timd2 } & Hsd3b5 & \\
\hline & & 6 \\
\hline & & 0.81467 \\
\hline \multirow[t]{3}{*}{ Timd2 } & Aox3 & \\
\hline & & 8 \\
\hline & & 0.87159 \\
\hline \multirow[t]{3}{*}{ Timd2 } & Smox & \\
\hline & & 8 \\
\hline & & 0.87364 \\
\hline \multirow[t]{2}{*}{ Timd2 } & LOC433886 & \\
\hline & & 5 \\
\hline \multirow[t]{2}{*}{ Timd2 } & Ngef & 0.90763 \\
\hline & & 0.76337 \\
\hline \multirow[t]{2}{*}{ Timd2 } & Zfp750 & \\
\hline & & 7 \\
\hline
\end{tabular}




\begin{tabular}{|c|c|c|}
\hline Timd2 & 1810020C02Rik & $\begin{array}{c}0.75128 \\
4\end{array}$ \\
\hline Timd2 & Slc41a3 & $\begin{array}{c}0.93239 \\
8\end{array}$ \\
\hline Timd2 & Sel1h & $\begin{array}{c}0.82285 \\
9\end{array}$ \\
\hline Timd2 & Fam129a & $\begin{array}{c}0.72780 \\
8\end{array}$ \\
\hline Timd2 & Ica1 & $\begin{array}{c}0.76261 \\
3\end{array}$ \\
\hline Timd2 & 4432416J03Rik & $\begin{array}{c}0.80631 \\
3\end{array}$ \\
\hline Timd2 & C430002P19Rik & $\begin{array}{c}0.86069 \\
9\end{array}$ \\
\hline Timd2 & Ces1 & $\begin{array}{c}0.76531 \\
8\end{array}$ \\
\hline Timd2 & Bves & 0.74963 \\
\hline Timd2 & D11Lgp2e & $\begin{array}{c}0.83565 \\
1\end{array}$ \\
\hline Timd2 & Bcdo2 & $\begin{array}{c}0.89807 \\
7\end{array}$ \\
\hline
\end{tabular}




\begin{tabular}{|c|c|c|}
\hline Timd2 & 5730410E15Rik & $\begin{array}{c}0.75893 \\
4\end{array}$ \\
\hline Timd2 & Gpr152 & $\begin{array}{c}0.71093 \\
1\end{array}$ \\
\hline Tnfrsf12a & Psat1 & $\begin{array}{c}0.85779 \\
3\end{array}$ \\
\hline Tnfrsf12a & Ptrh1 & $\begin{array}{c}0.85528 \\
6\end{array}$ \\
\hline Tnfrsf12a & Hyou1 & $\begin{array}{c}0.74699 \\
9\end{array}$ \\
\hline Tnfrsf12a & 6720458F09Rik & $\begin{array}{c}0.82866 \\
7\end{array}$ \\
\hline Tnfrsf12a & C1qtnf1 & $\begin{array}{c}0.72053 \\
5\end{array}$ \\
\hline Tnfrsf12a & Nol5a & $\begin{array}{c}0.72825 \\
5\end{array}$ \\
\hline Tnfrsf12a & Kdelr3 & $\begin{array}{c}0.90731 \\
4\end{array}$ \\
\hline Tnfrsf12a & Bcmo1 & $\begin{array}{c}0.97437 \\
4\end{array}$ \\
\hline Tnfrsf12a & Ttc39a & 0.8388 \\
\hline
\end{tabular}




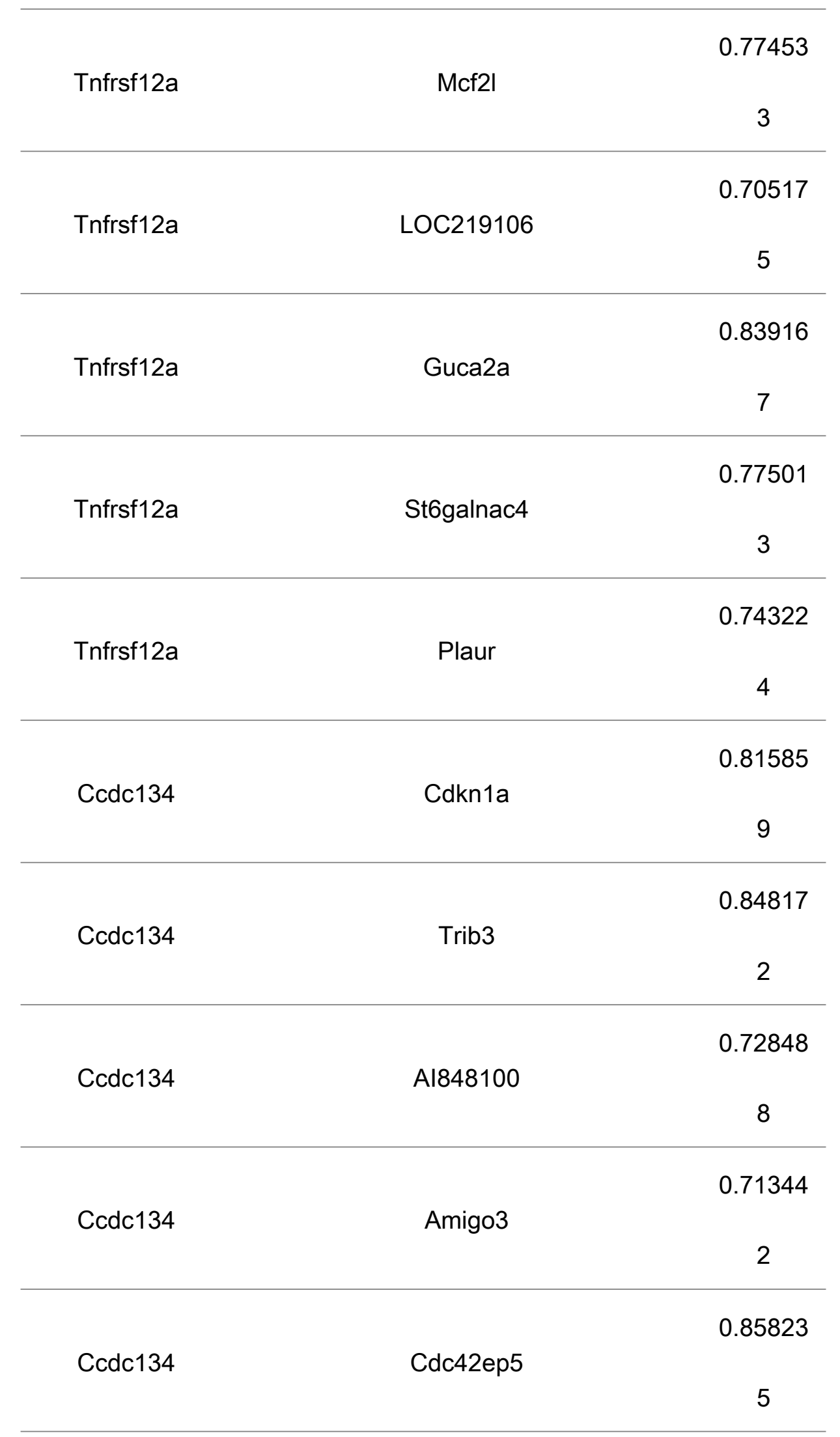




\begin{tabular}{|c|c|c|}
\hline Ccdc134 & B230217C12Rik & $\begin{array}{c}0.79997 \\
8\end{array}$ \\
\hline Ccdc134 & 4930581F22Rik & 0.7338 \\
\hline Ccdc134 & C730014E05Rik & $\begin{array}{c}0.74460 \\
2\end{array}$ \\
\hline Ccdc134 & A730014O07Rik & $\begin{array}{c}0.77994 \\
6\end{array}$ \\
\hline Ddah1 & Aldh8a1 & 0.91706 \\
\hline Ddah1 & Sucnr1 & $\begin{array}{c}0.71718 \\
8\end{array}$ \\
\hline Ddah1 & Lims2 & $\begin{array}{c}0.83700 \\
3\end{array}$ \\
\hline Ddah1 & Apoa5 & $\begin{array}{c}0.88466 \\
2\end{array}$ \\
\hline Ddah1 & EG381806 & $\begin{array}{c}0.89334 \\
7\end{array}$ \\
\hline Ddah1 & Cldn1 & $\begin{array}{c}0.81305 \\
4\end{array}$ \\
\hline Ddah1 & LOC380998 & $\begin{array}{c}0.90153 \\
5\end{array}$ \\
\hline
\end{tabular}




\begin{tabular}{|c|c|c|}
\hline Ddah1 & Bdh1 & $\begin{array}{c}0.87887 \\
7\end{array}$ \\
\hline Ddah1 & Dhcr7 & 0.8279 \\
\hline Ddah1 & Tmie & $\begin{array}{c}0.73962 \\
7\end{array}$ \\
\hline Ddah1 & Abca8a & $\begin{array}{c}0.75267 \\
4\end{array}$ \\
\hline Ddah1 & 3010026009Rik & $\begin{array}{c}0.97031 \\
1\end{array}$ \\
\hline Ddah1 & Paqr7 & $\begin{array}{c}0.77480 \\
6\end{array}$ \\
\hline Ddah1 & LOC100047427 & $\begin{array}{c}0.90506 \\
1\end{array}$ \\
\hline Ddah1 & Cdc14b & $\begin{array}{c}0.87539 \\
1\end{array}$ \\
\hline Ddah1 & Prss8 & $\begin{array}{c}0.89968 \\
7\end{array}$ \\
\hline Ddah1 & EG240549 & $\begin{array}{c}0.83495 \\
4\end{array}$ \\
\hline Ddah1 & 4432416J03Rik & $\begin{array}{c}0.72117 \\
1\end{array}$ \\
\hline
\end{tabular}




\begin{tabular}{|c|c|c|}
\hline \multirow[t]{2}{*}{ Ddah1 } & Slc27a3 & $\begin{array}{c}0.84193 \\
1\end{array}$ \\
\hline & & 0.79291 \\
\hline \multirow[t]{3}{*}{ Ddah1 } & D11Lgp2e & \\
\hline & & 5 \\
\hline & & 0.87622 \\
\hline \multirow[t]{3}{*}{ Ddah1 } & Zfp57 & \\
\hline & & 4 \\
\hline & & 0.75321 \\
\hline \multirow[t]{3}{*}{ Ddah1 } & 2610042O14Rik & \\
\hline & & 3 \\
\hline & & 0.71488 \\
\hline \multirow[t]{3}{*}{ Ddah1 } & 1810046K07Rik & \\
\hline & & 9 \\
\hline & & 0.74459 \\
\hline \multirow[t]{3}{*}{ Ddah1 } & Mpdz & \\
\hline & & 3 \\
\hline & & 0.88051 \\
\hline \multirow[t]{3}{*}{ Nans } & EG240327 & \\
\hline & & 5 \\
\hline & & 0.92404 \\
\hline \multirow[t]{3}{*}{ Nans } & 2810423A18Rik & \\
\hline & & 3 \\
\hline & & 0.90993 \\
\hline \multirow[t]{3}{*}{ Nans } & Derl3 & \\
\hline & & 3 \\
\hline & & 0.83181 \\
\hline \multirow[t]{2}{*}{ Nans } & Abcg5 & \\
\hline & & 1 \\
\hline
\end{tabular}




\begin{tabular}{|c|c|c|}
\hline \multirow[t]{2}{*}{ Nans } & Psen2 & $\begin{array}{c}0.76907 \\
6\end{array}$ \\
\hline & & 0.72427 \\
\hline \multirow[t]{3}{*}{ Nans } & Klkb1 & \\
\hline & & 6 \\
\hline & & 0.81912 \\
\hline \multirow[t]{3}{*}{ Nans } & Nalp6 & \\
\hline & & 3 \\
\hline & & 0.92737 \\
\hline \multirow[t]{3}{*}{ Nans } & Tlcd2 & \\
\hline & & 2 \\
\hline & & 0.72071 \\
\hline \multirow[t]{3}{*}{ Nans } & Aldh8a1 & \\
\hline & & 9 \\
\hline & & 0.73649 \\
\hline \multirow[t]{3}{*}{ Nans } & Cldn2 & \\
\hline & & 1 \\
\hline & & 0.88889 \\
\hline \multirow[t]{3}{*}{ Nans } & Acat2 & \\
\hline & & 2 \\
\hline & & 0.91585 \\
\hline \multirow[t]{3}{*}{ Nans } & Sucnr1 & \\
\hline & & 3 \\
\hline & & 0.79832 \\
\hline \multirow[t]{2}{*}{ Nans } & Lims2 & \\
\hline & & 9 \\
\hline \multirow[t]{2}{*}{ Nans } & Piga & 0.93198 \\
\hline & & 0.93113 \\
\hline \multirow[t]{2}{*}{ Nans } & 1700019H03Rik & \\
\hline & & 9 \\
\hline
\end{tabular}




\begin{tabular}{|c|c|c|}
\hline Nans & BC048644 & 0.90185 \\
\hline & & 0.79063 \\
\hline \multirow[t]{2}{*}{ Nans } & Abcg8 & \\
\hline & & 3 \\
\hline \multirow[t]{2}{*}{ Nans } & Proz & 0.93005 \\
\hline & & 0.80509 \\
\hline \multirow[t]{3}{*}{ Nans } & Apoa5 & \\
\hline & & 5 \\
\hline & & 0.91077 \\
\hline \multirow[t]{3}{*}{ Nans } & LOC240549 & \\
\hline & & 1 \\
\hline & & 0.97751 \\
\hline \multirow[t]{3}{*}{ Nans } & Ung & \\
\hline & & 9 \\
\hline & & 0.81096 \\
\hline \multirow[t]{3}{*}{ Nans } & Bdh1 & \\
\hline & & 9 \\
\hline & & 0.85590 \\
\hline \multirow[t]{3}{*}{ Nans } & Tmem25 & \\
\hline & & 2 \\
\hline & & 0.84583 \\
\hline \multirow[t]{3}{*}{ Nans } & Ccdc120 & \\
\hline & & 1 \\
\hline & & 0.82184 \\
\hline \multirow[t]{3}{*}{ Nans } & Dhcr7 & \\
\hline & & 4 \\
\hline & & 0.83180 \\
\hline \multirow[t]{2}{*}{ Nans } & Mist1 & \\
\hline & & 7 \\
\hline
\end{tabular}




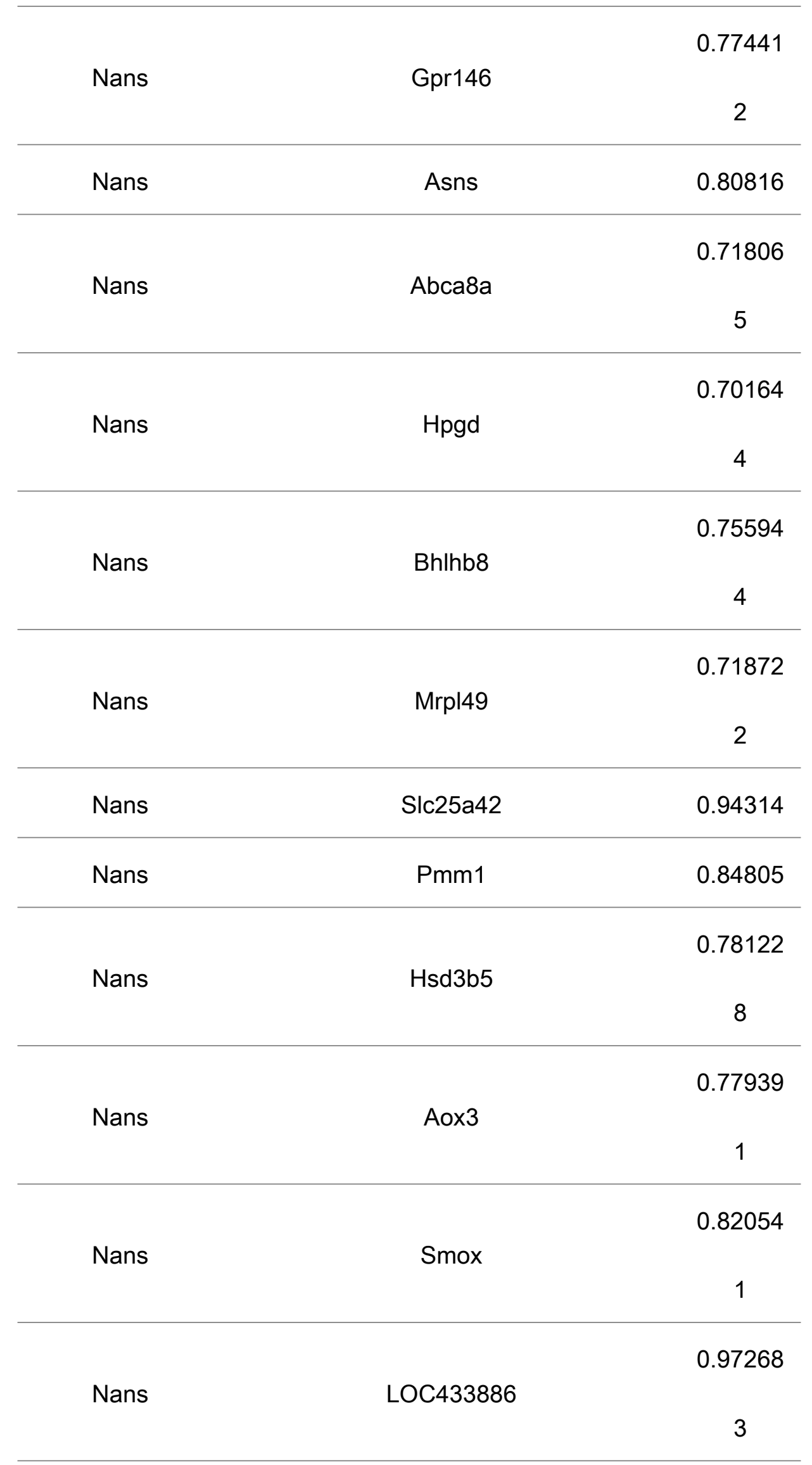




\begin{tabular}{|c|c|c|}
\hline \multirow[t]{2}{*}{ Nans } & Ngef & $\begin{array}{c}0.90288 \\
6\end{array}$ \\
\hline & & 0.73370 \\
\hline \multirow[t]{3}{*}{ Nans } & Zfp750 & \\
\hline & & 6 \\
\hline & & 0.71255 \\
\hline \multirow[t]{2}{*}{ Nans } & 1810020C02Rik & \\
\hline & & 9 \\
\hline \multirow[t]{2}{*}{ Nans } & Slc41a3 & 0.97094 \\
\hline & & 0.71828 \\
\hline \multirow[t]{3}{*}{ Nans } & Sel1h & \\
\hline & & 8 \\
\hline & & 0.75076 \\
\hline \multirow[t]{2}{*}{ Nans } & Ica1 & \\
\hline & & 3 \\
\hline \multirow[t]{2}{*}{ Nans } & Map3k6 & 0.73809 \\
\hline & & 0.81107 \\
\hline \multirow[t]{3}{*}{ Nans } & 4432416J03Rik & \\
\hline & & 9 \\
\hline & & 0.81957 \\
\hline \multirow[t]{3}{*}{ Nans } & C430002P19Rik & \\
\hline & & 5 \\
\hline & & 0.76595 \\
\hline \multirow[t]{3}{*}{ Nans } & Ces1 & \\
\hline & & 8 \\
\hline & & 0.76894 \\
\hline \multirow[t]{2}{*}{ Nans } & Gins2 & \\
\hline & & 1 \\
\hline
\end{tabular}




\begin{tabular}{|c|c|c|}
\hline \multirow[t]{2}{*}{ Nans } & D11Lgp2e & $\begin{array}{c}0.89511 \\
3\end{array}$ \\
\hline & & 0.89985 \\
\hline \multirow[t]{3}{*}{ Nans } & Bcdo2 & \\
\hline & & 7 \\
\hline & & 0.71359 \\
\hline \multirow[t]{3}{*}{ Nans } & Cnksr1 & \\
\hline & & 2 \\
\hline & & 0.74446 \\
\hline \multirow[t]{2}{*}{ Nans } & 2610042O14Rik & \\
\hline & & 4 \\
\hline \multirow[t]{2}{*}{ Nans } & B230207L18Rik & 0.70424 \\
\hline & & 0.70557 \\
\hline \multirow[t]{3}{*}{ Nans } & E030049G20Rik & \\
\hline & & 9 \\
\hline & & 0.82745 \\
\hline \multirow[t]{3}{*}{ Nans } & Gpr152 & \\
\hline & & 9 \\
\hline & & 0.73895 \\
\hline \multirow[t]{3}{*}{ AU018778 } & Abcg5 & \\
\hline & & 2 \\
\hline & & 0.74075 \\
\hline \multirow[t]{3}{*}{ AU018778 } & Hes6 & \\
\hline & & 9 \\
\hline & & 0.78289 \\
\hline \multirow[t]{3}{*}{ AU018778 } & Aldh8a1 & \\
\hline & & 7 \\
\hline & & 0.75268 \\
\hline \multirow[t]{2}{*}{ AU018778 } & Lims2 & \\
\hline & & 6 \\
\hline
\end{tabular}




\begin{tabular}{|c|c|c|}
\hline AU018778 & Pbld & $\begin{array}{c}0.94455 \\
1\end{array}$ \\
\hline AU018778 & Keg1 & $\begin{array}{c}0.96516 \\
8\end{array}$ \\
\hline AU018778 & Bdh1 & $\begin{array}{c}0.71637 \\
4\end{array}$ \\
\hline AU018778 & Tmem25 & $\begin{array}{c}0.74122 \\
4\end{array}$ \\
\hline AU018778 & Abca8a & $\begin{array}{c}0.79362 \\
8\end{array}$ \\
\hline AU018778 & Mrpl49 & $\begin{array}{c}0.72646 \\
2\end{array}$ \\
\hline AU018778 & Paqr7 & $\begin{array}{c}0.75573 \\
4\end{array}$ \\
\hline AU018778 & Tjp3 & $\begin{array}{c}0.79036 \\
4\end{array}$ \\
\hline AU018778 & Cabc1 & $\begin{array}{c}0.86024 \\
3\end{array}$ \\
\hline AU018778 & Slc44a3 & $\begin{array}{c}0.78861 \\
5\end{array}$ \\
\hline
\end{tabular}




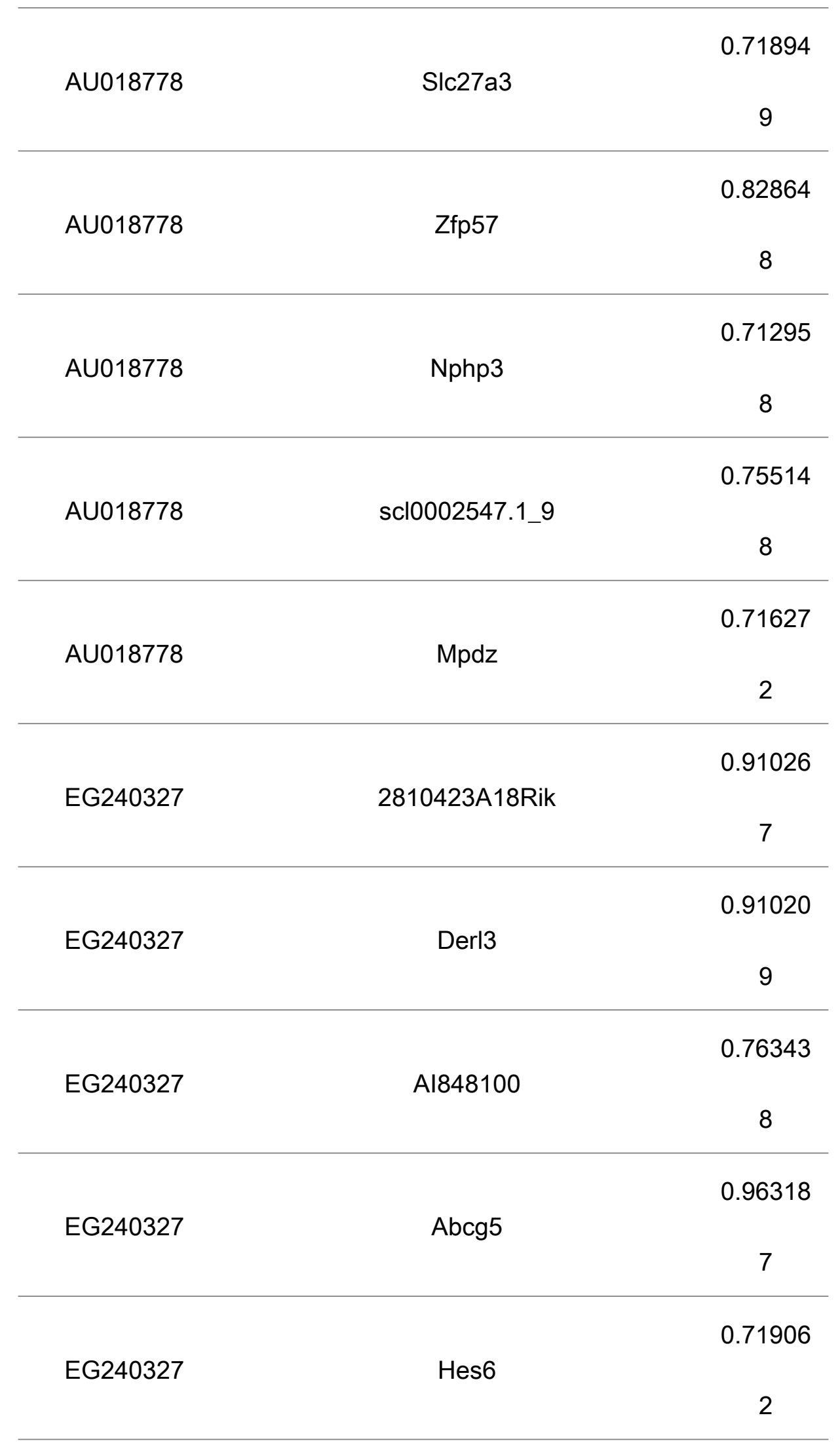




\begin{tabular}{|c|c|c|}
\hline EG240327 & Nalp6 & $\begin{array}{c}0.95197 \\
2\end{array}$ \\
\hline EG240327 & Tlcd2 & $\begin{array}{c}0.93471 \\
5\end{array}$ \\
\hline EG240327 & Aldh8a1 & $\begin{array}{c}0.81720 \\
8\end{array}$ \\
\hline EG240327 & Acat2 & $\begin{array}{c}0.81677 \\
8\end{array}$ \\
\hline EG240327 & Sucnr1 & $\begin{array}{c}0.91672 \\
4\end{array}$ \\
\hline EG240327 & Lims2 & $\begin{array}{c}0.90538 \\
8\end{array}$ \\
\hline EG240327 & Piga & 0.88536 \\
\hline EG240327 & 1700019H03Rik & $\begin{array}{c}0.71230 \\
3\end{array}$ \\
\hline EG240327 & Agxt2l1 & $\begin{array}{c}0.83554 \\
4\end{array}$ \\
\hline EG240327 & BC048644 & $\begin{array}{c}0.84855 \\
1\end{array}$ \\
\hline EG240327 & Proz & $\begin{array}{c}0.95774 \\
3\end{array}$ \\
\hline
\end{tabular}




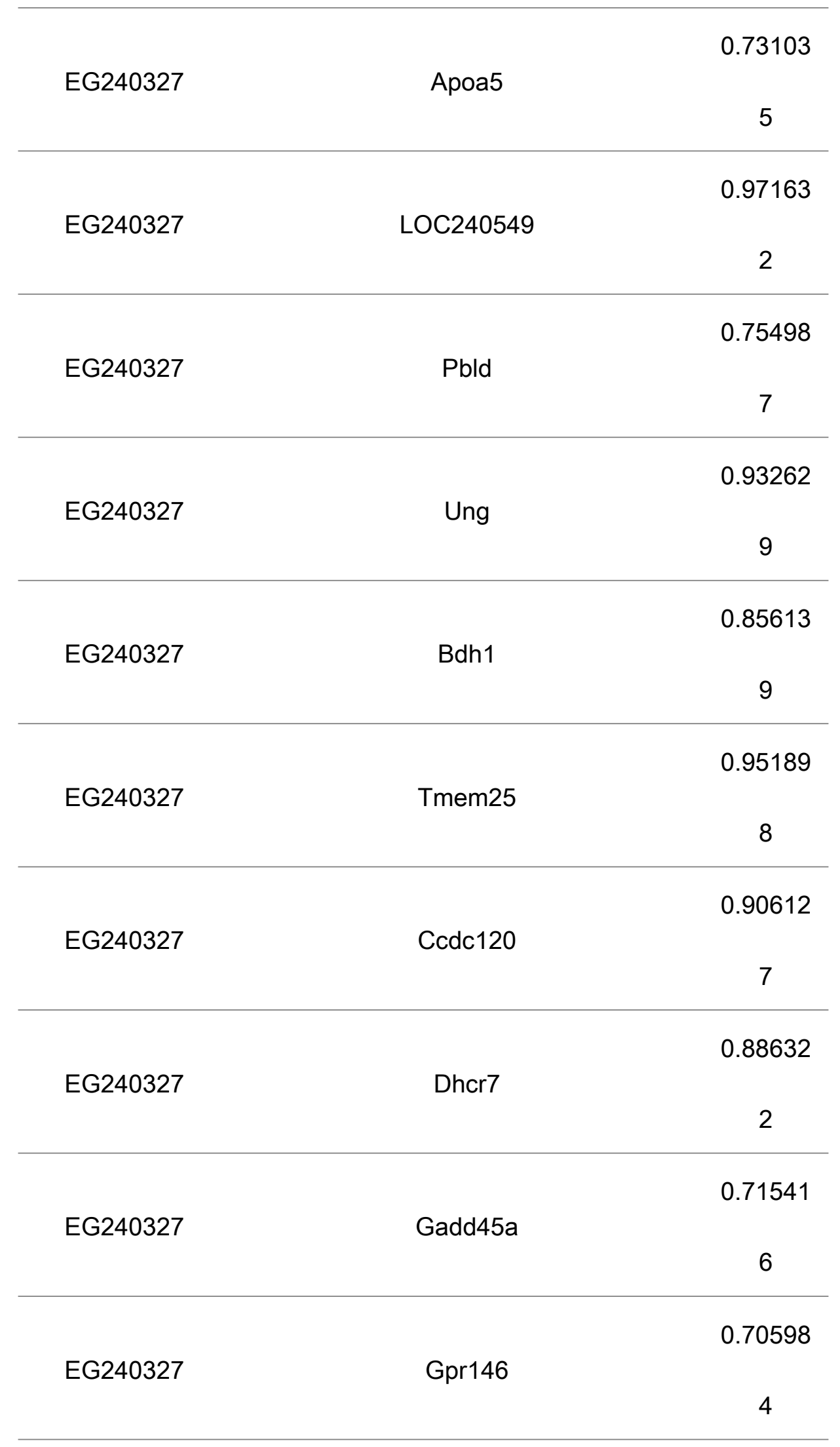




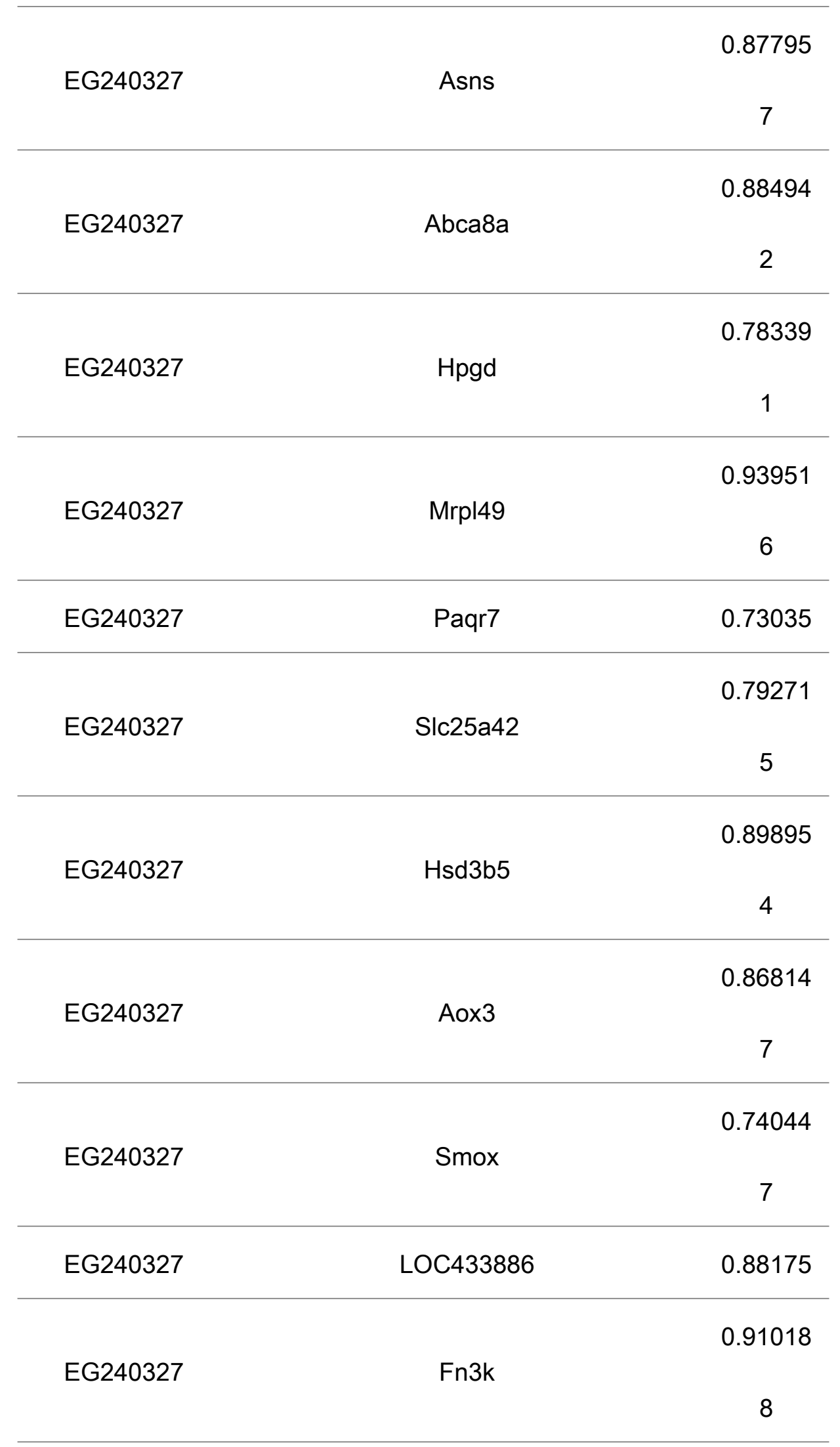




\begin{tabular}{|c|c|c|}
\hline EG240327 & Ngef & $\begin{array}{c}0.99402 \\
2\end{array}$ \\
\hline EG240327 & Zfp750 & $\begin{array}{c}0.93258 \\
1\end{array}$ \\
\hline EG240327 & Slc41a3 & $\begin{array}{c}0.88711 \\
2\end{array}$ \\
\hline EG240327 & Sel1h & $\begin{array}{c}0.74803 \\
3\end{array}$ \\
\hline EG240327 & Fam129a & $\begin{array}{c}0.90690 \\
9\end{array}$ \\
\hline EG240327 & Ica1 & $\begin{array}{c}0.82055 \\
6\end{array}$ \\
\hline EG240327 & Slc27a3 & $\begin{array}{c}0.83937 \\
7\end{array}$ \\
\hline EG240327 & B430005K18Rik & 0.7547 \\
\hline EG240327 & Ces1 & $\begin{array}{c}0.90673 \\
1\end{array}$ \\
\hline EG240327 & Gins2 & $\begin{array}{c}0.75795 \\
3\end{array}$ \\
\hline EG240327 & D11Lgp2e & $\begin{array}{c}0.78747 \\
5\end{array}$ \\
\hline
\end{tabular}




\begin{tabular}{|c|c|c|}
\hline & & 0.73555 \\
\hline \multirow[t]{2}{*}{ EG240327 } & D930009C14Rik & \\
\hline & & 2 \\
\hline & & 0.84030 \\
\hline \multirow[t]{3}{*}{ EG240327 } & Bcdo2 & \\
\hline & & 7 \\
\hline & & 0.78894 \\
\hline \multirow[t]{3}{*}{ EG240327 } & Rgs12 & \\
\hline & & 4 \\
\hline & & 0.75514 \\
\hline \multirow[t]{3}{*}{ EG240327 } & 5730410E15Rik & \\
\hline & & 3 \\
\hline & & 0.72601 \\
\hline \multirow[t]{3}{*}{ EG240327 } & E030049G20Rik & \\
\hline & & 6 \\
\hline & & 0.85712 \\
\hline \multirow[t]{3}{*}{ EG240327 } & Gpr152 & \\
\hline & & 4 \\
\hline & & 0.72353 \\
\hline \multirow[t]{3}{*}{ Cdkn1a } & 2810423A18Rik & \\
\hline & & 7 \\
\hline & & 0.95008 \\
\hline \multirow[t]{3}{*}{ Cdkn1a } & Trib3 & \\
\hline & & 8 \\
\hline & & 0.78687 \\
\hline \multirow[t]{3}{*}{ Cdkn1a } & Al848100 & \\
\hline & & 1 \\
\hline & & 0.78260 \\
\hline \multirow[t]{2}{*}{ Cdkn1a } & Piga & \\
\hline & & 8 \\
\hline
\end{tabular}




\begin{tabular}{|c|c|c|}
\hline Cdkn1a & Amigo3 & $\begin{array}{c}0.97210 \\
1\end{array}$ \\
\hline \multirow{3}{*}{ Cdkn1a } & \multirow{3}{*}{ Cdc42ep5 } & 0.83582 \\
\hline & & \\
\hline & & 6 \\
\hline \multirow[t]{2}{*}{ Cdkn1a } & Slc41a3 & 0.72996 \\
\hline & & 0.83975 \\
\hline \multirow[t]{3}{*}{ Cdkn1a } & Map3k6 & \\
\hline & & 3 \\
\hline & & 0.80937 \\
\hline \multirow[t]{3}{*}{ Cdkn1a } & Gins2 & \\
\hline & & 4 \\
\hline & & 0.71273 \\
\hline \multirow[t]{3}{*}{ Cdkn1a } & B230207L18Rik & \\
\hline & & 7 \\
\hline & & 0.86858 \\
\hline \multirow[t]{3}{*}{ 2810423A18Rik } & Derl3 & \\
\hline & & 8 \\
\hline & & 0.75923 \\
\hline \multirow[t]{3}{*}{ 2810423A18Rik } & Al848100 & \\
\hline & & 4 \\
\hline & & 0.86441 \\
\hline \multirow[t]{3}{*}{ 2810423A18Rik } & Abcg5 & \\
\hline & & 9 \\
\hline & & 0.84112 \\
\hline \multirow[t]{2}{*}{ 2810423A18Rik } & Nalp6 & \\
\hline & & 5 \\
\hline 2810423A18Rik & Tlcd2 & 0.87134 \\
\hline
\end{tabular}




\begin{tabular}{|c|c|c|}
\hline 2810423A18Rik & Acat2 & $\begin{array}{c}0.77402 \\
1\end{array}$ \\
\hline 2810423A18Rik & Sucnr1 & $\begin{array}{c}0.80592 \\
4\end{array}$ \\
\hline 2810423A18Rik & Lims2 & $\begin{array}{c}0.74217 \\
8\end{array}$ \\
\hline 2810423A18Rik & Piga & $\begin{array}{c}0.99077 \\
1\end{array}$ \\
\hline 2810423A18Rik & 1700019H03Rik & $\begin{array}{c}0.83735 \\
8\end{array}$ \\
\hline 2810423A18Rik & Agxt2l1 & $\begin{array}{c}0.76106 \\
6\end{array}$ \\
\hline 2810423A18Rik & BC048644 & $\begin{array}{c}0.84315 \\
7\end{array}$ \\
\hline 2810423A18Rik & Amigo3 & $\begin{array}{c}0.71797 \\
1\end{array}$ \\
\hline 2810423A18Rik & Proz & $\begin{array}{c}0.86022 \\
3\end{array}$ \\
\hline 2810423A18Rik & LOC240549 & $\begin{array}{c}0.95112 \\
9\end{array}$ \\
\hline
\end{tabular}




\begin{tabular}{|c|c|c|}
\hline 2810423A18Rik & Ung & $\begin{array}{c}0.90674 \\
6\end{array}$ \\
\hline 2810423A18Rik & Bdh1 & $\begin{array}{c}0.72941 \\
9\end{array}$ \\
\hline 2810423A18Rik & Tmem25 & $\begin{array}{c}0.86372 \\
4\end{array}$ \\
\hline 2810423A18Rik & Ccdc120 & $\begin{array}{c}0.76904 \\
8\end{array}$ \\
\hline 2810423A18Rik & Dhcr7 & $\begin{array}{c}0.72603 \\
3\end{array}$ \\
\hline 2810423A18Rik & Mist1 & $\begin{array}{c}0.78526 \\
1\end{array}$ \\
\hline 2810423A18Rik & Gpr146 & $\begin{array}{c}0.72968 \\
8\end{array}$ \\
\hline 2810423A18Rik & Asns & $\begin{array}{c}0.74312 \\
5\end{array}$ \\
\hline 2810423A18Rik & Bhlhb8 & $\begin{array}{c}0.74490 \\
2\end{array}$ \\
\hline 2810423A18Rik & Mrpl49 & $\begin{array}{c}0.78964 \\
2\end{array}$ \\
\hline
\end{tabular}




\begin{tabular}{|c|c|c|}
\hline 2810423A18Rik & Slc25a42 & $\begin{array}{c}0.84902 \\
8\end{array}$ \\
\hline 2810423A18Rik & Hsd3b5 & $\begin{array}{c}0.81690 \\
8\end{array}$ \\
\hline 2810423A18Rik & LOC433886 & $\begin{array}{c}0.94060 \\
3\end{array}$ \\
\hline 2810423A18Rik & Fn3k & $\begin{array}{c}0.79415 \\
5\end{array}$ \\
\hline 2810423A18Rik & Ngef & $\begin{array}{c}0.90489 \\
8\end{array}$ \\
\hline 2810423A18Rik & Zfp750 & $\begin{array}{c}0.78815 \\
8\end{array}$ \\
\hline 2810423A18Rik & Slc41a3 & $\begin{array}{c}0.94509 \\
1\end{array}$ \\
\hline 2810423A18Rik & Fam129a & $\begin{array}{c}0.72464 \\
3\end{array}$ \\
\hline 2810423A18Rik & Ica1 & $\begin{array}{c}0.72907 \\
7\end{array}$ \\
\hline 2810423A18Rik & Map3k6 & $\begin{array}{c}0.75597 \\
6\end{array}$ \\
\hline
\end{tabular}




\begin{tabular}{|c|c|c|}
\hline 2810423A18Rik & Slc27a3 & $\begin{array}{c}0.70028 \\
2\end{array}$ \\
\hline 2810423A18Rik & Ces1 & $\begin{array}{c}0.84218 \\
6\end{array}$ \\
\hline 2810423A18Rik & Gins2 & $\begin{array}{c}0.81398 \\
3\end{array}$ \\
\hline 2810423A18Rik & D11Lgp2e & $\begin{array}{c}0.75999 \\
9\end{array}$ \\
\hline 2810423A18Rik & Bcdo2 & $\begin{array}{c}0.75379 \\
4\end{array}$ \\
\hline 2810423A18Rik & Rgs12 & $\begin{array}{c}0.74725 \\
1\end{array}$ \\
\hline 2810423A18Rik & E030049G20Rik & $\begin{array}{c}0.71490 \\
7\end{array}$ \\
\hline 2810423A18Rik & Gpr152 & $\begin{array}{c}0.95844 \\
3\end{array}$ \\
\hline Trib3 & Amigo3 & $\begin{array}{c}0.92546 \\
8\end{array}$ \\
\hline Trib3 & Cdc42ep5 & $\begin{array}{c}0.85238 \\
2\end{array}$ \\
\hline
\end{tabular}




\begin{tabular}{|c|c|c|}
\hline \multirow[t]{2}{*}{ Trib3 } & Hisppd2a & $\begin{array}{c}0.84436 \\
5\end{array}$ \\
\hline & & 0.80243 \\
\hline \multirow[t]{3}{*}{ Trib3 } & DXBwg1396e & \\
\hline & & 8 \\
\hline & & 0.81754 \\
\hline \multirow[t]{3}{*}{ Derl3 } & 6430548M08Rik & \\
\hline & & 9 \\
\hline & & 0.88313 \\
\hline \multirow[t]{3}{*}{ Derl3 } & Al848100 & \\
\hline & & 9 \\
\hline & & 0.79128 \\
\hline \multirow[t]{3}{*}{ Derl3 } & Abcg5 & \\
\hline & & 1 \\
\hline & & 0.72965 \\
\hline \multirow[t]{3}{*}{ Derl3 } & Psen2 & \\
\hline & & 5 \\
\hline & & 0.70114 \\
\hline \multirow[t]{3}{*}{ Derl3 } & Klkb1 & \\
\hline & & 5 \\
\hline & & 0.74612 \\
\hline \multirow[t]{3}{*}{ Derl3 } & Aldh18a1 & \\
\hline & & 3 \\
\hline & & 0.83461 \\
\hline \multirow[t]{3}{*}{ Derl3 } & Nalp6 & \\
\hline & & 6 \\
\hline & & 0.91709 \\
\hline \multirow[t]{2}{*}{ Derl3 } & Tlcd2 & \\
\hline & & 2 \\
\hline
\end{tabular}




\begin{tabular}{|c|c|c|}
\hline \multirow[t]{2}{*}{ Derl3 } & Aldh8a1 & $\begin{array}{c}0.70460 \\
1\end{array}$ \\
\hline & & 0.72462 \\
\hline \multirow[t]{3}{*}{ Derl3 } & Cldn2 & \\
\hline & & 6 \\
\hline & & 0.96879 \\
\hline \multirow[t]{3}{*}{ Derl3 } & Acat2 & \\
\hline & & 1 \\
\hline & & 0.88979 \\
\hline \multirow[t]{3}{*}{ Derl3 } & Sucnr1 & \\
\hline & & 5 \\
\hline & & 0.80685 \\
\hline \multirow[t]{3}{*}{ Derl3 } & Lims2 & \\
\hline & & 8 \\
\hline & & 0.89143 \\
\hline \multirow[t]{3}{*}{ Derl3 } & Piga & \\
\hline & & 2 \\
\hline & & 0.73104 \\
\hline \multirow[t]{3}{*}{ Derl3 } & 1700019H03Rik & \\
\hline & & 2 \\
\hline & & 0.86170 \\
\hline \multirow[t]{3}{*}{ Derl3 } & BC048644 & \\
\hline & & 1 \\
\hline & & 0.77311 \\
\hline \multirow[t]{3}{*}{ Derl3 } & Abcg8 & \\
\hline & & 5 \\
\hline & & 0.94368 \\
\hline \multirow[t]{2}{*}{ Derl3 } & Proz & \\
\hline & & 7 \\
\hline
\end{tabular}




\begin{tabular}{|c|c|c|}
\hline \multirow[t]{2}{*}{ Derl3 } & Apoa5 & $\begin{array}{c}0.72576 \\
1\end{array}$ \\
\hline & & 0.87504 \\
\hline \multirow[t]{3}{*}{ Derl3 } & LOC240549 & \\
\hline & & 6 \\
\hline & & 0.96677 \\
\hline \multirow[t]{3}{*}{ Derl3 } & Ung & \\
\hline & & 2 \\
\hline & & 0.76521 \\
\hline \multirow[t]{3}{*}{ Derl3 } & Bdh1 & \\
\hline & & 4 \\
\hline & & 0.79162 \\
\hline \multirow[t]{3}{*}{ Derl3 } & Tmem25 & \\
\hline & & 9 \\
\hline & & 0.88486 \\
\hline \multirow[t]{3}{*}{ Derl3 } & Ccdc120 & \\
\hline & & 6 \\
\hline & & 0.83052 \\
\hline \multirow[t]{3}{*}{ Derl3 } & Dhcr7 & \\
\hline & & 5 \\
\hline & & 0.73654 \\
\hline \multirow[t]{3}{*}{ Derl3 } & Gadd45a & \\
\hline & & 6 \\
\hline & & 0.76355 \\
\hline \multirow[t]{3}{*}{ Derl3 } & Gpr146 & \\
\hline & & 8 \\
\hline & & 0.94460 \\
\hline \multirow[t]{2}{*}{ Derl3 } & Asns & \\
\hline & & 8 \\
\hline
\end{tabular}




\begin{tabular}{|c|c|c|}
\hline \multirow[t]{2}{*}{ Derl3 } & Abca8a & $\begin{array}{c}0.74445 \\
5\end{array}$ \\
\hline & & 0.91777 \\
\hline \multirow[t]{3}{*}{ Derl3 } & Hpgd & \\
\hline & & 4 \\
\hline & & 0.75882 \\
\hline \multirow[t]{3}{*}{ Derl3 } & Mrpl49 & \\
\hline & & 9 \\
\hline & & 0.82312 \\
\hline \multirow[t]{3}{*}{ Derl3 } & Slc25a42 & \\
\hline & & 6 \\
\hline & & 0.81276 \\
\hline \multirow[t]{3}{*}{ Derl3 } & Hsd3b5 & \\
\hline & & 4 \\
\hline & & 0.76142 \\
\hline \multirow[t]{3}{*}{ Derl3 } & Aox3 & \\
\hline & & 2 \\
\hline & & 0.71058 \\
\hline \multirow[t]{3}{*}{ Derl3 } & Smox & \\
\hline & & 5 \\
\hline & & 0.84995 \\
\hline \multirow[t]{3}{*}{ Derl3 } & LOC433886 & \\
\hline & & 5 \\
\hline & & 0.71215 \\
\hline \multirow[t]{3}{*}{ Derl3 } & Fn3k & \\
\hline & & 6 \\
\hline & & 0.94439 \\
\hline \multirow[t]{2}{*}{ Derl3 } & Ngef & \\
\hline & & 8 \\
\hline
\end{tabular}




\begin{tabular}{|c|c|c|}
\hline \multirow[t]{2}{*}{ Derl3 } & Zfp750 & $\begin{array}{c}0.77833 \\
1\end{array}$ \\
\hline & & 0.70446 \\
\hline \multirow[t]{3}{*}{ Derl3 } & Pdzk1ip1 & \\
\hline & & 1 \\
\hline & & 0.95715 \\
\hline \multirow[t]{3}{*}{ Derl3 } & Slc41a3 & \\
\hline & & 5 \\
\hline & & 0.91996 \\
\hline \multirow[t]{3}{*}{ Derl3 } & Sel1h & \\
\hline & & 5 \\
\hline & & 0.74359 \\
\hline \multirow[t]{3}{*}{ Derl3 } & Fam129a & \\
\hline & & 9 \\
\hline & & 0.91330 \\
\hline \multirow[t]{3}{*}{ Derl3 } & Ica1 & \\
\hline & & 1 \\
\hline & & 0.72847 \\
\hline \multirow[t]{3}{*}{ Derl3 } & Map3k6 & \\
\hline & & 6 \\
\hline & & 0.83493 \\
\hline \multirow[t]{3}{*}{ Derl3 } & 4432416J03Rik & \\
\hline & & 1 \\
\hline & & 0.78333 \\
\hline \multirow[t]{3}{*}{ Derl3 } & LOC242997 & \\
\hline & & 7 \\
\hline & & 0.78321 \\
\hline \multirow[t]{2}{*}{ Derl3 } & Slc27a3 & \\
\hline & & 6 \\
\hline
\end{tabular}




\begin{tabular}{|c|c|c|}
\hline Derl3 & C430002P19Rik & $\begin{array}{c}0.77893 \\
8\end{array}$ \\
\hline & & 0.74762 \\
\hline \multirow[t]{3}{*}{ Derl3 } & Ces1 & \\
\hline & & 3 \\
\hline & & 0.76466 \\
\hline \multirow[t]{3}{*}{ Derl3 } & Gins2 & \\
\hline & & 5 \\
\hline & & 0.82313 \\
\hline \multirow[t]{3}{*}{ Derl3 } & D11Lgp2e & \\
\hline & & 9 \\
\hline & & 0.73523 \\
\hline \multirow[t]{2}{*}{ Derl3 } & D930009C14Rik & \\
\hline & & 3 \\
\hline \multirow[t]{2}{*}{ Derl3 } & Bcdo2 & 0.79823 \\
\hline & & 0.70229 \\
\hline \multirow[t]{3}{*}{ Derl3 } & 5730410E15Rik & \\
\hline & & 4 \\
\hline & & 0.73469 \\
\hline \multirow[t]{3}{*}{ Derl3 } & B230207L18Rik & \\
\hline & & 4 \\
\hline & & 0.72768 \\
\hline \multirow[t]{2}{*}{ Derl3 } & Gpr152 & \\
\hline & & 5 \\
\hline \multirow[t]{2}{*}{ 6430548M08Rik } & Al848100 & 0.75171 \\
\hline & & 0.81173 \\
\hline \multirow[t]{2}{*}{ 6430548M08Rik } & Aldh18a1 & \\
\hline & & 9 \\
\hline
\end{tabular}




\begin{tabular}{|c|c|c|}
\hline 6430548M08Rik & Tlcd2 & $\begin{array}{c}0.71652 \\
1\end{array}$ \\
\hline 6430548M08Rik & Acat2 & 0.81175 \\
\hline 6430548M08Rik & Proz & $\begin{array}{c}0.75298 \\
1\end{array}$ \\
\hline 6430548M08Rik & Ung & $\begin{array}{c}0.71069 \\
6\end{array}$ \\
\hline 6430548M08Rik & Ccdc120 & 0.7862 \\
\hline 6430548M08Rik & $\mathrm{Hr}$ & $\begin{array}{c}0.77951 \\
2\end{array}$ \\
\hline 6430548M08Rik & Gadd45a & $\begin{array}{c}0.88208 \\
8\end{array}$ \\
\hline 6430548M08Rik & Mthfd2 & $\begin{array}{c}0.83351 \\
7\end{array}$ \\
\hline 6430548M08Rik & Asns & $\begin{array}{c}0.86174 \\
8\end{array}$ \\
\hline 6430548M08Rik & Hpgd & $\begin{array}{c}0.95804 \\
9\end{array}$ \\
\hline 6430548M08Rik & Ngef & $\begin{array}{c}0.74074 \\
7\end{array}$ \\
\hline
\end{tabular}




\begin{tabular}{|c|c|c|}
\hline 6430548M08Rik & A630034I12Rik & $\begin{array}{c}0.80476 \\
2\end{array}$ \\
\hline 6430548M08Rik & Sel1h & 0.94116 \\
\hline 6430548M08Rik & Niban & 0.83023 \\
\hline 6430548M08Rik & Ica1 & $\begin{array}{c}0.80366 \\
3\end{array}$ \\
\hline 6430548M08Rik & LOC242997 & $\begin{array}{c}0.81559 \\
4\end{array}$ \\
\hline 6430548M08Rik & Zbtb32 & $\begin{array}{c}0.75782 \\
4\end{array}$ \\
\hline 6430548M08Rik & 1810015A11Rik & $\begin{array}{c}0.80347 \\
5\end{array}$ \\
\hline 6430548M08Rik & Bves & $\begin{array}{c}0.78138 \\
1\end{array}$ \\
\hline 6430548M08Rik & D930009C14Rik & $\begin{array}{c}0.82596 \\
3\end{array}$ \\
\hline 6430548M08Rik & 5730410E15Rik & $\begin{array}{c}0.70213 \\
6\end{array}$ \\
\hline 6430548M08Rik & 1810008K04Rik & $\begin{array}{c}0.73088 \\
2\end{array}$ \\
\hline
\end{tabular}




\begin{tabular}{|c|c|c|}
\hline 6430548M08Rik & 5830411E10Rik & $\begin{array}{c}0.73624 \\
4\end{array}$ \\
\hline 8430408G22Rik & Selenbp2 & $\begin{array}{c}0.70531 \\
9\end{array}$ \\
\hline 8430408G22Rik & Acy3 & $\begin{array}{c}0.96311 \\
7\end{array}$ \\
\hline 8430408G22Rik & Olig1 & $\begin{array}{c}0.80155 \\
2\end{array}$ \\
\hline 8430408G22Rik & LOC673607 & $\begin{array}{c}0.90264 \\
5\end{array}$ \\
\hline 8430408G22Rik & Abca2 & $\begin{array}{c}0.74206 \\
4\end{array}$ \\
\hline 8430408G22Rik & $\mathrm{Nfe} 2$ & $\begin{array}{c}0.94236 \\
2\end{array}$ \\
\hline 8430408G22Rik & Zc3h12d & $\begin{array}{c}0.77908 \\
1\end{array}$ \\
\hline Upp2 & Acacb & $\begin{array}{c}0.72137 \\
1\end{array}$ \\
\hline Upp2 & Spata2L & $\begin{array}{c}0.80300 \\
6\end{array}$ \\
\hline
\end{tabular}




\begin{tabular}{|c|c|c|}
\hline \multirow[t]{2}{*}{ Upp2 } & Dclk3 & $\begin{array}{c}0.81889 \\
1\end{array}$ \\
\hline & & 0.77992 \\
\hline \multirow[t]{3}{*}{ Upp2 } & Ncald & \\
\hline & & 4 \\
\hline & & 0.92024 \\
\hline \multirow[t]{3}{*}{ Al848100 } & Aldh18a1 & \\
\hline & & 3 \\
\hline & & 0.81736 \\
\hline \multirow[t]{3}{*}{ Al848100 } & Acat2 & \\
\hline & & 2 \\
\hline & & 0.80140 \\
\hline \multirow[t]{3}{*}{ Al848100 } & Piga & \\
\hline & & 2 \\
\hline & & 0.72529 \\
\hline \multirow[t]{3}{*}{ Al848100 } & Proz & \\
\hline & & 7 \\
\hline & & 0.70215 \\
\hline \multirow[t]{3}{*}{ Al848100 } & LOC240549 & \\
\hline & & 6 \\
\hline & & 0.76924 \\
\hline \multirow[t]{3}{*}{ Al848100 } & Ung & \\
\hline & & 2 \\
\hline & & 0.78448 \\
\hline \multirow[t]{3}{*}{ Al848100 } & Gadd45a & \\
\hline & & 6 \\
\hline & & 0.83401 \\
\hline \multirow[t]{2}{*}{ Al848100 } & Asns & \\
\hline & & 4 \\
\hline Al848100 & Hpgd & 0.8937 \\
\hline
\end{tabular}




\begin{tabular}{|c|c|c|}
\hline \multirow[t]{2}{*}{ Al848100 } & Ngef & $\begin{array}{c}0.78661 \\
3\end{array}$ \\
\hline & & 0.70193 \\
\hline \multirow[t]{2}{*}{ Al848100 } & Cdc42ep5 & \\
\hline & & 9 \\
\hline \multirow[t]{2}{*}{ Al848100 } & Slc41a3 & 0.82424 \\
\hline & & 0.87878 \\
\hline \multirow[t]{3}{*}{ Al848100 } & Sel1h & \\
\hline & & 9 \\
\hline & & 0.90659 \\
\hline \multirow[t]{3}{*}{ Al848100 } & Ica1 & \\
\hline & & 4 \\
\hline & & 0.73292 \\
\hline \multirow[t]{3}{*}{ Al848100 } & Map3k6 & \\
\hline & & 1 \\
\hline & & 0.91257 \\
\hline \multirow[t]{3}{*}{ Al848100 } & LOC242997 & \\
\hline & & 8 \\
\hline & & 0.76919 \\
\hline \multirow[t]{3}{*}{ Al848100 } & Zbtb32 & \\
\hline & & 1 \\
\hline & & 0.72063 \\
\hline \multirow[t]{3}{*}{ Al848100 } & Slc27a3 & \\
\hline & & 2 \\
\hline & & 0.75655 \\
\hline \multirow[t]{3}{*}{ Al848100 } & Gins2 & \\
\hline & & 3 \\
\hline & & 0.76697 \\
\hline \multirow[t]{2}{*}{ Al848100 } & 4930581F22Rik & \\
\hline & & 4 \\
\hline
\end{tabular}




\begin{tabular}{|c|c|c|}
\hline Al848100 & D930009C14Rik & $\begin{array}{c}0.73496 \\
5\end{array}$ \\
\hline \multirow{3}{*}{ Al848100 } & & 0.79646 \\
\hline & C730014E05Rik & \\
\hline & & 9 \\
\hline \multirow{3}{*}{ Al848100 } & & 0.82012 \\
\hline & Rock2 & \\
\hline & & 6 \\
\hline \multirow{3}{*}{ Al848100 } & & 0.70298 \\
\hline & B230207L18Rik & \\
\hline & & 5 \\
\hline \multirow[t]{2}{*}{ A530050D06Rik } & Psen2 & 0.95192 \\
\hline & & 0.95572 \\
\hline \multirow[t]{2}{*}{ A530050D06Rik } & Klkb1 & \\
\hline & & 9 \\
\hline \multirow[t]{2}{*}{ A530050D06Rik } & Cldn2 & 0.96477 \\
\hline & & 0.73242 \\
\hline \multirow[t]{3}{*}{ A530050D06Rik } & Acat2 & \\
\hline & & 7 \\
\hline & & 0.71351 \\
\hline \multirow[t]{3}{*}{ A530050D06Rik } & Sucnr1 & \\
\hline & & 4 \\
\hline & & 0.99037 \\
\hline \multirow[t]{3}{*}{ A530050D06Rik } & 1110046J11Rik & \\
\hline & & 2 \\
\hline & & 0.88704 \\
\hline \multirow[t]{2}{*}{ A530050D06Rik } & Abcg8 & \\
\hline & & 9 \\
\hline
\end{tabular}




\begin{tabular}{|c|c|c|}
\hline A530050D06Rik & Apoa5 & $\begin{array}{c}0.87122 \\
6\end{array}$ \\
\hline A530050D06Rik & C730029A08Rik & $\begin{array}{c}0.98033 \\
9\end{array}$ \\
\hline A530050D06Rik & LOC380998 & $\begin{array}{c}0.73535 \\
2\end{array}$ \\
\hline A530050D06Rik & Bdh1 & $\begin{array}{c}0.70482 \\
8\end{array}$ \\
\hline A530050D06Rik & Dhcr7 & $\begin{array}{c}0.70456 \\
9\end{array}$ \\
\hline A530050D06Rik & 3010026009Rik & $\begin{array}{c}0.78005 \\
7\end{array}$ \\
\hline A530050D06Rik & Pdzk1ip1 & $\begin{array}{c}0.91491 \\
4\end{array}$ \\
\hline A530050D06Rik & Cpsf4l & $\begin{array}{c}0.73516 \\
3\end{array}$ \\
\hline A530050D06Rik & EG240549 & $\begin{array}{c}0.74381 \\
7\end{array}$ \\
\hline A530050D06Rik & 4432416J03Rik & 0.91222 \\
\hline A530050D06Rik & 2610528J11Rik & $\begin{array}{c}0.75347 \\
4\end{array}$ \\
\hline
\end{tabular}




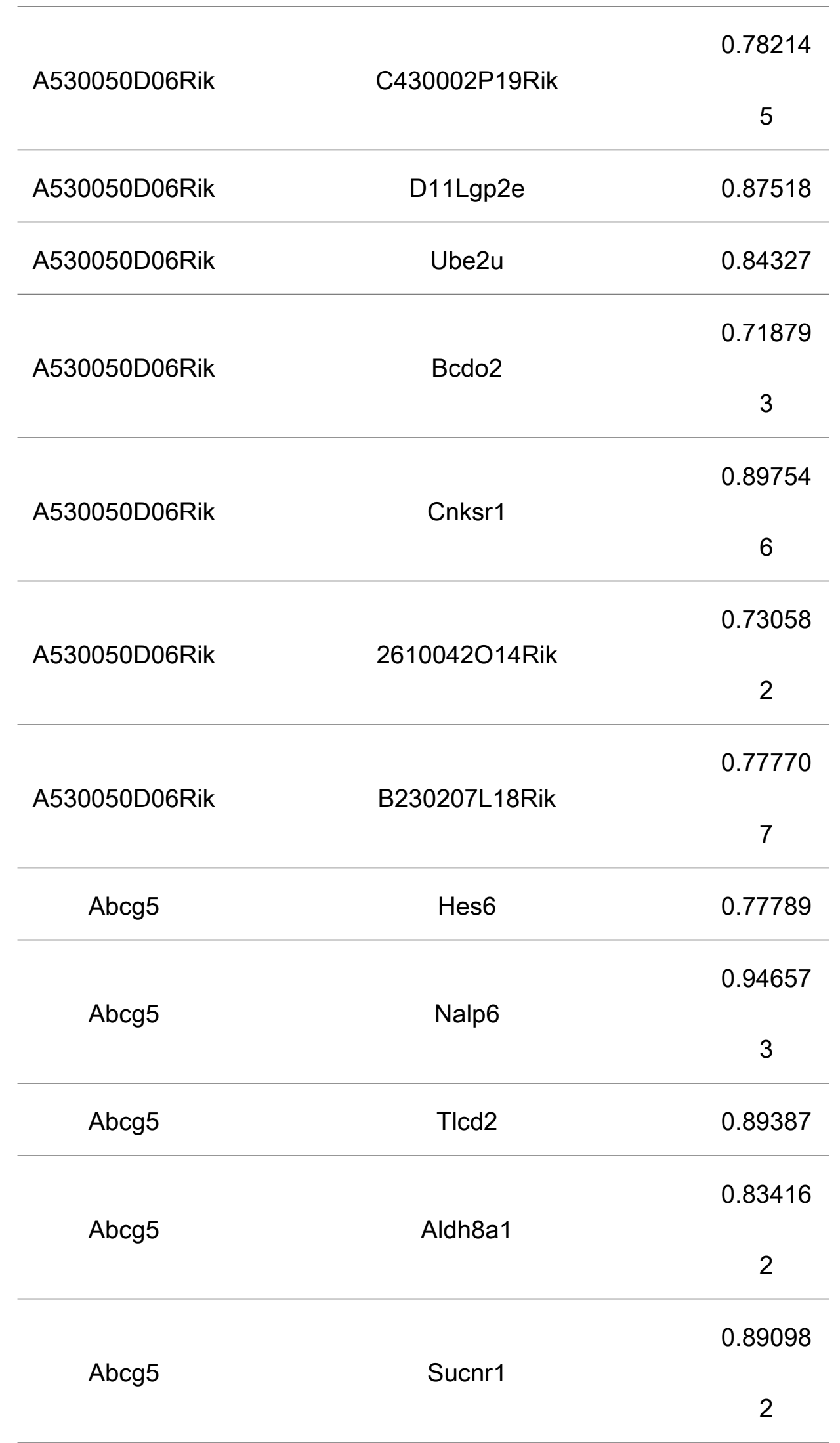




\begin{tabular}{|c|c|c|}
\hline Abcg5 & Lims2 & $\begin{array}{c}0.90043 \\
4\end{array}$ \\
\hline & & 0.81476 \\
\hline \multirow[t]{2}{*}{ Abcg5 } & Piga & \\
\hline & & 9 \\
\hline \multirow[t]{2}{*}{ Abcg5 } & 1700019H03Rik & 0.70034 \\
\hline & & 0.86238 \\
\hline \multirow[t]{3}{*}{ Abcg5 } & Agxt211 & \\
\hline & & 9 \\
\hline & & 0.80624 \\
\hline \multirow[t]{3}{*}{ Abcg5 } & BC048644 & \\
\hline & & 6 \\
\hline & & 0.90768 \\
\hline \multirow[t]{3}{*}{ Abcg5 } & Proz & \\
\hline & & 6 \\
\hline & & 0.71514 \\
\hline \multirow[t]{3}{*}{ Abcg5 } & Apoa5 & \\
\hline & & 7 \\
\hline & & 0.95794 \\
\hline \multirow[t]{3}{*}{ Abcg5 } & LOC240549 & \\
\hline & & 6 \\
\hline & & 0.85921 \\
\hline \multirow[t]{2}{*}{ Abcg5 } & Pbld & \\
\hline & & 4 \\
\hline \multirow[t]{2}{*}{ Abcg5 } & Ung & 0.86237 \\
\hline & & 0.86744 \\
\hline \multirow[t]{2}{*}{ Abcg5 } & Bdh1 & \\
\hline & & 6 \\
\hline
\end{tabular}




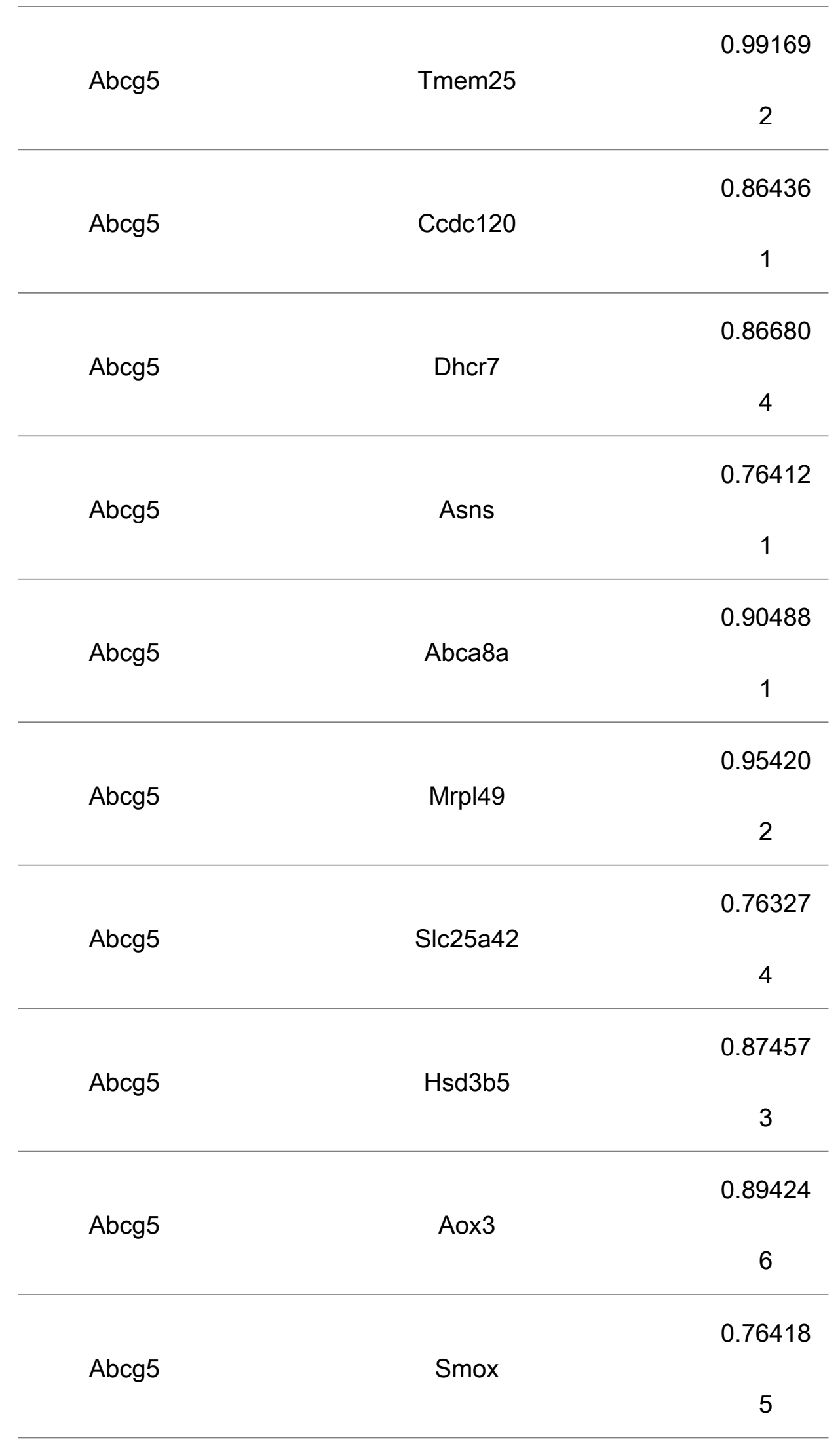




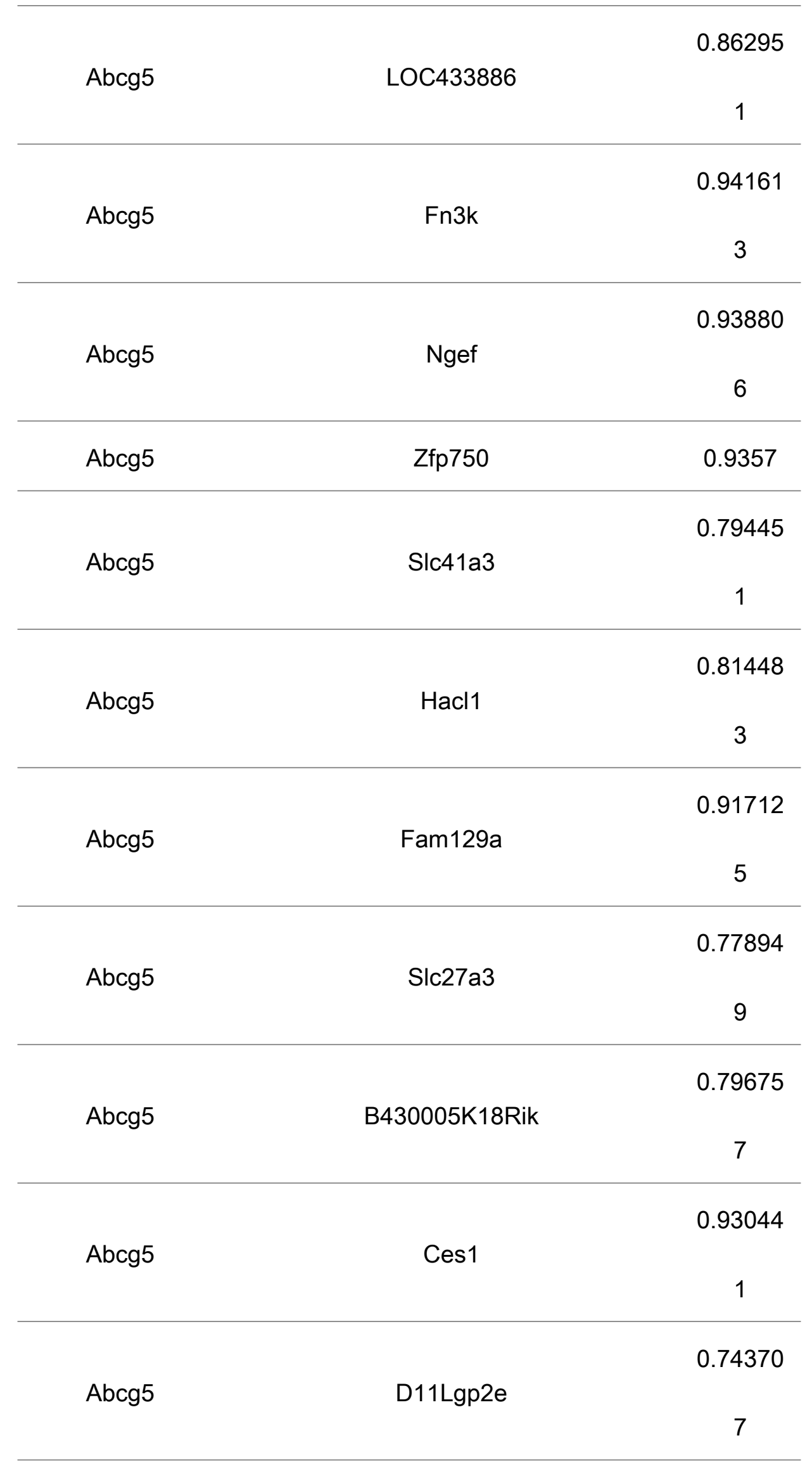




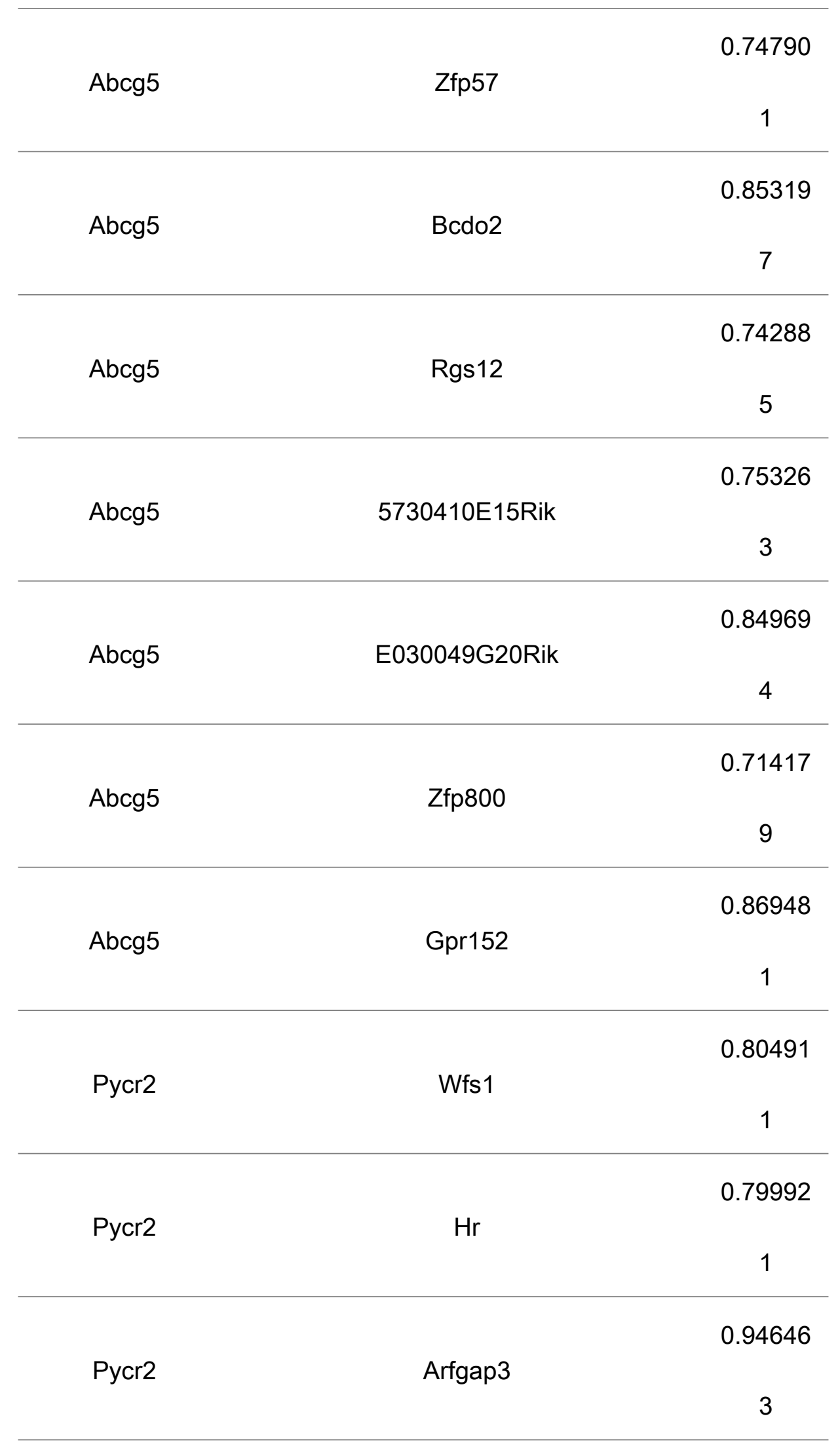




\begin{tabular}{|c|c|c|}
\hline Pycr2 & Cyb5r1 & $\begin{array}{c}0.79564 \\
9\end{array}$ \\
\hline Pycr2 & Far1 & $\begin{array}{c}0.79212 \\
5\end{array}$ \\
\hline Pycr2 & Guca2a & $\begin{array}{c}0.85596 \\
1\end{array}$ \\
\hline Pycr2 & Bves & $\begin{array}{c}0.78399 \\
3\end{array}$ \\
\hline Pycr2 & 5730410E15Rik & $\begin{array}{c}0.73814 \\
6\end{array}$ \\
\hline Hes6 & Selenbp2 & $\begin{array}{c}0.85409 \\
1\end{array}$ \\
\hline Hes6 & Nalp6 & $\begin{array}{c}0.75524 \\
1\end{array}$ \\
\hline Hes6 & Agxt2l1 & $\begin{array}{c}0.94400 \\
6\end{array}$ \\
\hline Hes6 & LOC240549 & $\begin{array}{c}0.70080 \\
2\end{array}$ \\
\hline Hes6 & Pbld & $\begin{array}{c}0.83087 \\
8\end{array}$ \\
\hline
\end{tabular}




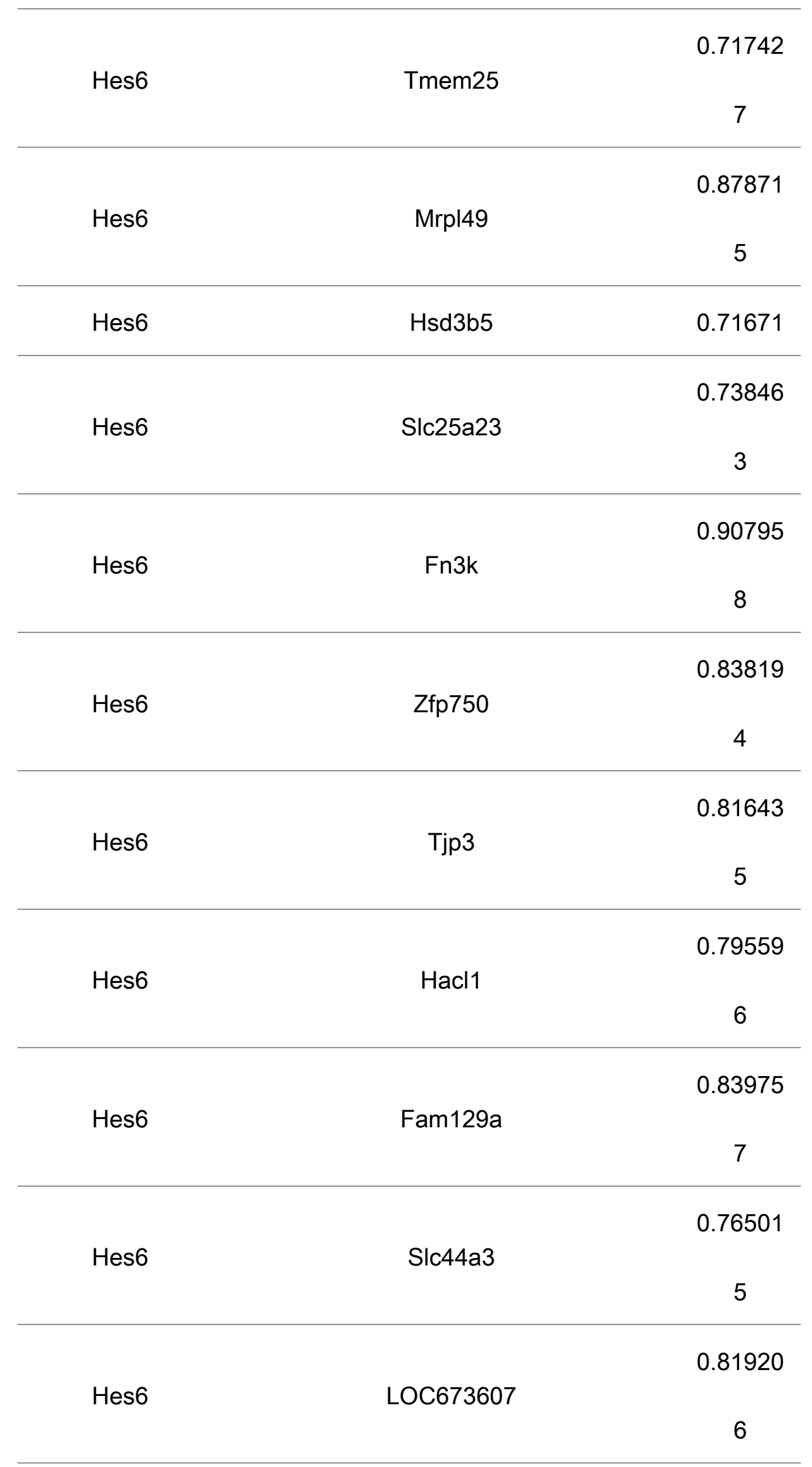




\begin{tabular}{|c|c|c|}
\hline \multirow[t]{2}{*}{ Hes6 } & Abca2 & $\begin{array}{c}0.86997 \\
7\end{array}$ \\
\hline & & 0.84762 \\
\hline \multirow[t]{3}{*}{ Hes6 } & B430005K18Rik & \\
\hline & & 5 \\
\hline & & 0.76034 \\
\hline \multirow[t]{3}{*}{ Hes6 } & Zc3h12d & \\
\hline & & 9 \\
\hline & & 0.81210 \\
\hline \multirow[t]{3}{*}{ Hes6 } & Ces1 & \\
\hline & & 5 \\
\hline & & 0.81805 \\
\hline \multirow[t]{3}{*}{ Hes6 } & Rgs12 & \\
\hline & & 2 \\
\hline & & 0.74856 \\
\hline \multirow[t]{3}{*}{ Wfs 1} & Nalp6 & \\
\hline & & 9 \\
\hline & & 0.78941 \\
\hline \multirow[t]{3}{*}{ Wfs 1} & Tlcd2 & \\
\hline & & 9 \\
\hline & & 0.80390 \\
\hline \multirow[t]{3}{*}{ Wfs 1} & Sucnr1 & \\
\hline & & 8 \\
\hline & & 0.71180 \\
\hline \multirow[t]{3}{*}{ Wfs 1} & Lims2 & \\
\hline & & 9 \\
\hline & & 0.86059 \\
\hline \multirow[t]{2}{*}{ Wfs 1} & Psat1 & \\
\hline & & 1 \\
\hline
\end{tabular}




\begin{tabular}{|c|c|c|}
\hline \multirow[t]{2}{*}{ Wfs 1} & Ptrh1 & $\begin{array}{c}0.81334 \\
7\end{array}$ \\
\hline & & 0.73808 \\
\hline \multirow[t]{3}{*}{ Wfs1 } & BC048644 & \\
\hline & & 3 \\
\hline & & 0.79023 \\
\hline \multirow[t]{3}{*}{ Wfs 1} & Proz & \\
\hline & & 5 \\
\hline & & 0.70958 \\
\hline \multirow[t]{3}{*}{ Wfs 1} & C1qtnf1 & \\
\hline & & 2 \\
\hline & & 0.75639 \\
\hline \multirow[t]{3}{*}{ Wfs 1} & Cox6a2 & \\
\hline & & 2 \\
\hline & & 0.87323 \\
\hline \multirow[t]{3}{*}{ Wfs 1} & Ccdc120 & \\
\hline & & 6 \\
\hline & & 0.76231 \\
\hline \multirow[t]{2}{*}{ Wfs 1} & Dhcr7 & \\
\hline & & 2 \\
\hline \multirow[t]{2}{*}{ Wfs 1} & $\mathrm{Hr}$ & 0.73517 \\
\hline & & 0.77455 \\
\hline \multirow[t]{3}{*}{ Wfs 1} & Abca8a & \\
\hline & & 6 \\
\hline & & 0.85379 \\
\hline \multirow[t]{3}{*}{ Wfs 1} & Arfgap3 & \\
\hline & & 7 \\
\hline & & 0.73058 \\
\hline \multirow[t]{2}{*}{ Wfs 1} & Pmm1 & \\
\hline & & 3 \\
\hline
\end{tabular}




\begin{tabular}{|c|c|c|}
\hline Wfs1 & Hsd3b5 & 0.70739 \\
\hline & & 0.88695 \\
\hline \multirow[t]{3}{*}{ Wfs1 } & Aox3 & \\
\hline & & 7 \\
\hline & & 0.90453 \\
\hline \multirow[t]{3}{*}{ Wfs 1} & Smox & \\
\hline & & 9 \\
\hline & & 0.70063 \\
\hline \multirow[t]{3}{*}{ Wfs 1} & Ngef & \\
\hline & & 8 \\
\hline & & 0.70202 \\
\hline \multirow[t]{3}{*}{ Wfs 1} & Zfp750 & \\
\hline & & 3 \\
\hline & & 0.82108 \\
\hline \multirow[t]{2}{*}{ Wfs 1} & 1810020C02Rik & \\
\hline & & 4 \\
\hline \multirow[t]{2}{*}{ Wfs 1} & Fam129a & 0.72865 \\
\hline & & 0.79184 \\
\hline \multirow[t]{3}{*}{ Wfs 1} & Mcf2I & \\
\hline & & 8 \\
\hline & & 0.78604 \\
\hline \multirow[t]{3}{*}{ Wfs 1} & Guca2a & \\
\hline & & 5 \\
\hline & & 0.82873 \\
\hline \multirow[t]{3}{*}{ Wfs 1} & 4833403D03Rik & \\
\hline & & 4 \\
\hline & & 0.92802 \\
\hline \multirow[t]{2}{*}{ Wfs 1} & Bves & \\
\hline & & 7 \\
\hline
\end{tabular}




\begin{tabular}{|c|c|c|}
\hline \multirow[t]{2}{*}{ Wfs 1} & Bcdo2 & $\begin{array}{c}0.82810 \\
8\end{array}$ \\
\hline & & 0.94792 \\
\hline \multirow[t]{3}{*}{ Wfs 1} & 5730410E15Rik & \\
\hline & & 1 \\
\hline & & 0.70034 \\
\hline \multirow[t]{3}{*}{ Wfs 1} & Zfp800 & \\
\hline & & 3 \\
\hline & & 0.73358 \\
\hline \multirow[t]{3}{*}{ Selenbp2 } & Nalp6 & \\
\hline & & 5 \\
\hline & & 0.86705 \\
\hline \multirow[t]{3}{*}{ Selenbp2 } & Agxt2l1 & \\
\hline & & 3 \\
\hline & & 0.78649 \\
\hline \multirow[t]{3}{*}{ Selenbp2 } & Mrpl49 & \\
\hline & & 4 \\
\hline & & 0.77202 \\
\hline \multirow[t]{3}{*}{ Selenbp2 } & Hsd3b5 & \\
\hline & & 6 \\
\hline & & 0.90559 \\
\hline \multirow[t]{3}{*}{ Selenbp2 } & Slc25a23 & \\
\hline & & 7 \\
\hline & & 0.82414 \\
\hline \multirow[t]{3}{*}{ Selenbp2 } & Fn3k & \\
\hline & & 7 \\
\hline & & 0.83501 \\
\hline \multirow[t]{2}{*}{ Selenbp2 } & LOC384022 & \\
\hline & & 7 \\
\hline
\end{tabular}




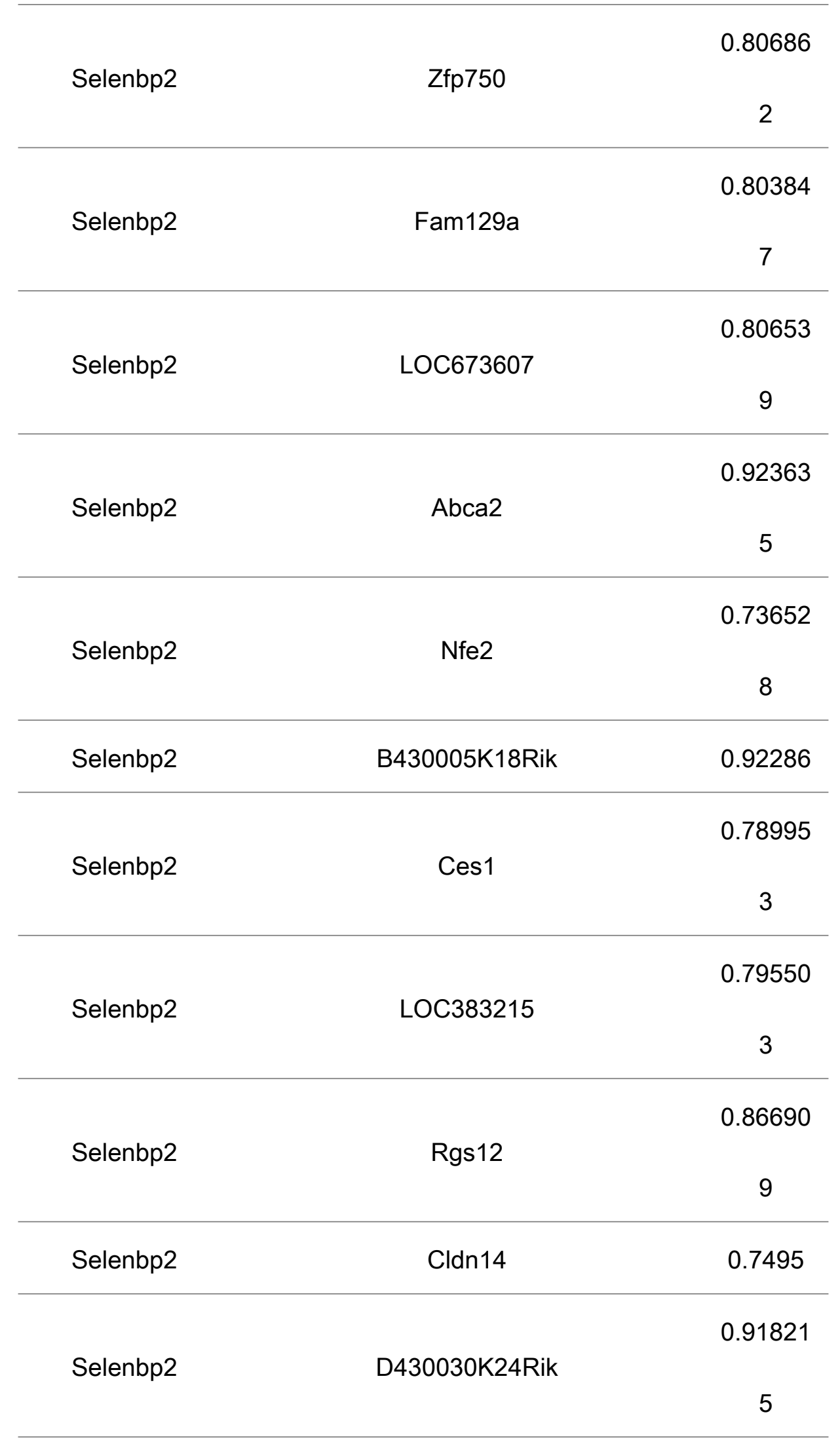




\begin{tabular}{|c|c|c|}
\hline \multirow[t]{2}{*}{ Psen2 } & Klkb1 & $\begin{array}{c}0.99550 \\
6\end{array}$ \\
\hline & & 0.93340 \\
\hline \multirow[t]{3}{*}{ Psen2 } & Cldn2 & \\
\hline & & 3 \\
\hline & & 0.84005 \\
\hline \multirow[t]{3}{*}{ Psen2 } & Acat2 & \\
\hline & & 3 \\
\hline & & 0.82357 \\
\hline \multirow[t]{3}{*}{ Psen2 } & Sucnr1 & \\
\hline & & 7 \\
\hline & & 0.71254 \\
\hline \multirow[t]{3}{*}{ Psen2 } & Lims2 & \\
\hline & & 2 \\
\hline & & 0.90951 \\
\hline \multirow[t]{3}{*}{ Psen2 } & 1110046J11Rik & \\
\hline & & 6 \\
\hline & & 0.71183 \\
\hline \multirow[t]{3}{*}{ Psen2 } & 1700019H03Rik & \\
\hline & & 5 \\
\hline & & 0.96879 \\
\hline \multirow[t]{3}{*}{ Psen2 } & Abcg8 & \\
\hline & & 1 \\
\hline & & 0.75513 \\
\hline \multirow[t]{3}{*}{ Psen2 } & Proz & \\
\hline & & 8 \\
\hline & & 0.86278 \\
\hline \multirow[t]{2}{*}{ Psen2 } & Apoa5 & \\
\hline & & 4 \\
\hline
\end{tabular}




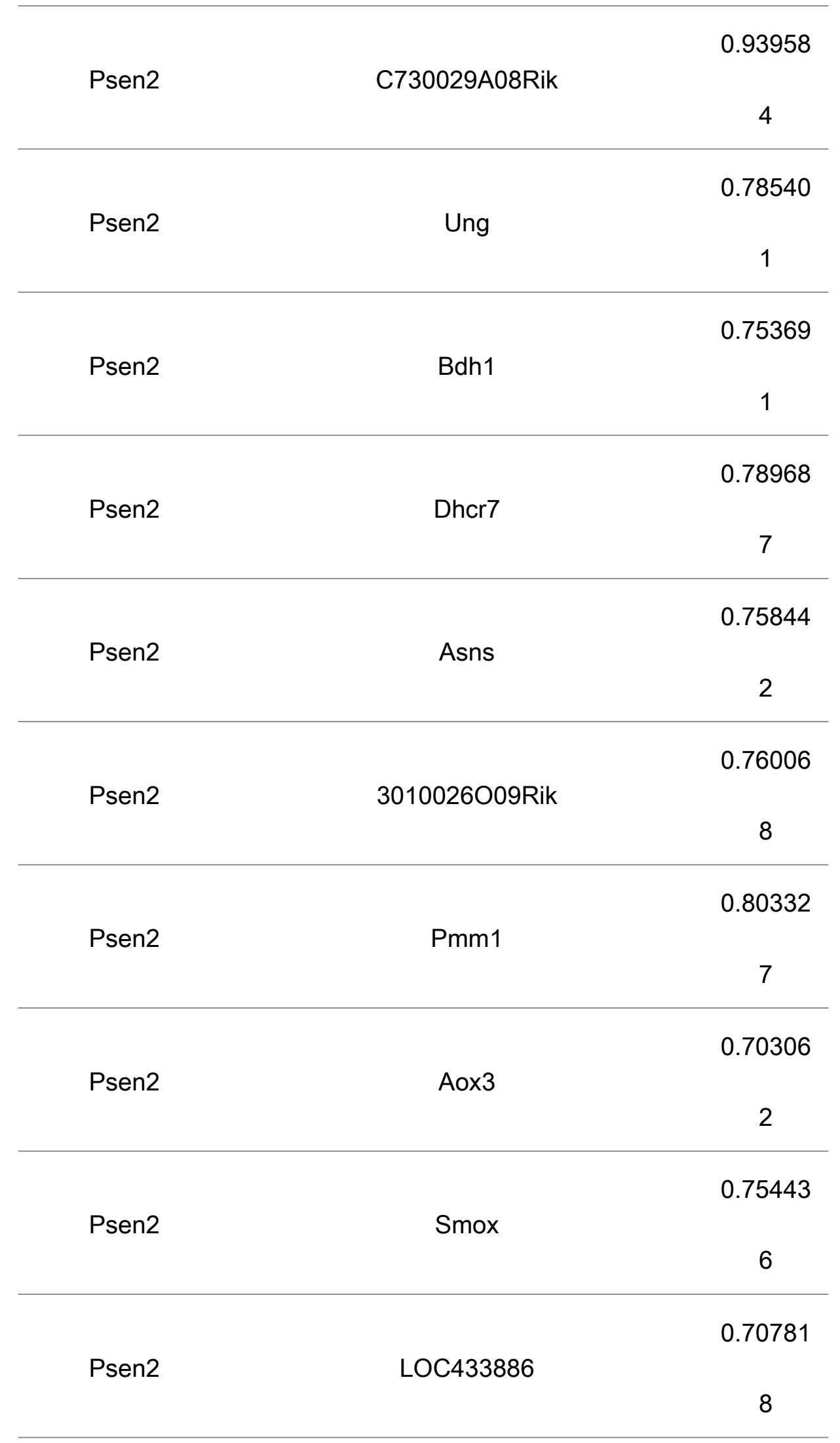




\begin{tabular}{|c|c|c|}
\hline \multirow[t]{2}{*}{ Psen2 } & Pdzk1ip1 & $\begin{array}{c}0.91711 \\
9\end{array}$ \\
\hline & & 0.71451 \\
\hline \multirow[t]{3}{*}{ Psen2 } & Slc41a3 & \\
\hline & & 5 \\
\hline & & 0.92969 \\
\hline \multirow[t]{3}{*}{ Psen2 } & 4432416J03Rik & \\
\hline & & 1 \\
\hline & & 0.87136 \\
\hline \multirow[t]{3}{*}{ Psen2 } & C430002P19Rik & \\
\hline & & 5 \\
\hline & & 0.89206 \\
\hline \multirow[t]{3}{*}{ Psen2 } & D11Lgp2e & \\
\hline & & 2 \\
\hline & & 0.90279 \\
\hline \multirow[t]{3}{*}{ Psen2 } & Ube2u & \\
\hline & & 4 \\
\hline & & 0.82817 \\
\hline \multirow[t]{3}{*}{ Psen2 } & Bcdo2 & \\
\hline & & 5 \\
\hline & & 0.86795 \\
\hline \multirow[t]{3}{*}{ Psen2 } & Cnksr1 & \\
\hline & & 6 \\
\hline & & 0.70270 \\
\hline \multirow[t]{3}{*}{ Psen2 } & 5730410E15Rik & \\
\hline & & 3 \\
\hline & & 0.73476 \\
\hline \multirow[t]{2}{*}{ Psen2 } & B230207L18Rik & \\
\hline & & 6 \\
\hline
\end{tabular}




\begin{tabular}{|c|c|c|}
\hline \multirow[t]{2}{*}{ Klkb1 } & Cldn2 & $\begin{array}{c}0.93244 \\
7\end{array}$ \\
\hline & & 0.81678 \\
\hline \multirow[t]{3}{*}{ Klkb1 } & Acat2 & \\
\hline & & 8 \\
\hline & & 0.80103 \\
\hline \multirow[t]{2}{*}{ Klkb1 } & Sucnr1 & \\
\hline & & 8 \\
\hline \multirow[t]{2}{*}{ Klkb1 } & Lims2 & 0.70432 \\
\hline & & 0.91252 \\
\hline \multirow[t]{3}{*}{ Klkb1 } & 1110046J11Rik & \\
\hline & & 3 \\
\hline & & 0.95208 \\
\hline \multirow[t]{3}{*}{ Klkb1 } & Abcg8 & \\
\hline & & 3 \\
\hline & & 0.72644 \\
\hline \multirow[t]{3}{*}{ Klkb1 } & Proz & \\
\hline & & 9 \\
\hline & & 0.85537 \\
\hline \multirow[t]{3}{*}{ Klkb1 } & Apoa5 & \\
\hline & & 7 \\
\hline & & 0.93182 \\
\hline \multirow[t]{3}{*}{ Klkb1 } & C730029A08Rik & \\
\hline & & 4 \\
\hline & & 0.75018 \\
\hline \multirow[t]{3}{*}{ Klkb1 } & Ung & \\
\hline & & 4 \\
\hline & & 0.74381 \\
\hline \multirow[t]{2}{*}{ Klkb1 } & Bdh1 & \\
\hline & & 6 \\
\hline
\end{tabular}




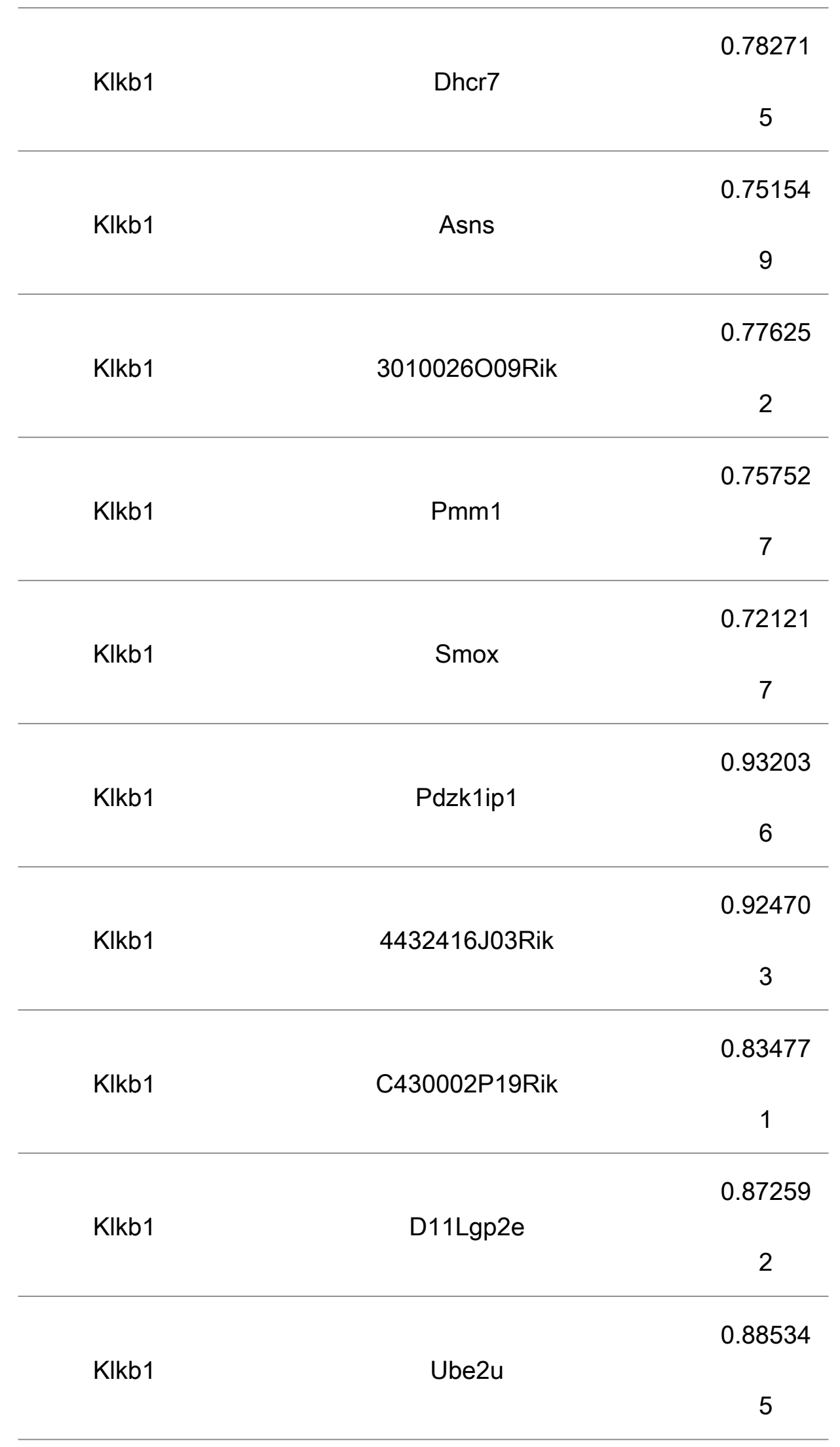




\begin{tabular}{|c|c|c|}
\hline \multirow[t]{2}{*}{ Klkb1 } & Bcdo2 & $\begin{array}{c}0.79991 \\
5\end{array}$ \\
\hline & & 0.83860 \\
\hline \multirow[t]{3}{*}{ Klkb1 } & Cnksr1 & \\
\hline & & 5 \\
\hline & & 0.70215 \\
\hline \multirow[t]{3}{*}{ Klkb1 } & 5730410E15Rik & \\
\hline & & 4 \\
\hline & & 0.72062 \\
\hline \multirow[t]{3}{*}{ Klkb1 } & B230207L18Rik & \\
\hline & & 5 \\
\hline & & 0.85579 \\
\hline \multirow[t]{3}{*}{ Aldh18a1 } & Gadd45a & \\
\hline & & 8 \\
\hline & & 0.75710 \\
\hline \multirow[t]{3}{*}{ Aldh18a1 } & Asns & \\
\hline & & 2 \\
\hline & & 0.89157 \\
\hline \multirow[t]{3}{*}{ Aldh18a1 } & Hpgd & \\
\hline & & 2 \\
\hline & & 0.86407 \\
\hline \multirow[t]{3}{*}{ Aldh18a1 } & Sel1h & \\
\hline & & 7 \\
\hline & & 0.83283 \\
\hline \multirow[t]{3}{*}{ Aldh18a1 } & Ica1 & \\
\hline & & 1 \\
\hline & & 0.96095 \\
\hline \multirow[t]{2}{*}{ Aldh18a1 } & LOC242997 & \\
\hline & & 3 \\
\hline
\end{tabular}




\begin{tabular}{|c|c|c|}
\hline \multirow[t]{2}{*}{ Aldh18a1 } & Zbtb32 & $\begin{array}{c}0.89937 \\
3\end{array}$ \\
\hline & & 0.75202 \\
\hline \multirow[t]{3}{*}{ Aldh18a1 } & 1810015A11Rik & \\
\hline & & 4 \\
\hline & & 0.73591 \\
\hline \multirow[t]{3}{*}{ Aldh18a1 } & 4930581F22Rik & \\
\hline & & 3 \\
\hline & & 0.77420 \\
\hline \multirow[t]{3}{*}{ Aldh18a1 } & D930009C14Rik & \\
\hline & & 4 \\
\hline & & 0.76499 \\
\hline \multirow[t]{2}{*}{ Aldh18a1 } & C730014E05Rik & \\
\hline & & 1 \\
\hline \multirow[t]{2}{*}{ Aldh18a1 } & Rock2 & 0.73253 \\
\hline & & 0.95190 \\
\hline \multirow[t]{3}{*}{ Nalp6 } & Tlcd2 & \\
\hline & & 9 \\
\hline & & 0.70855 \\
\hline \multirow[t]{3}{*}{ Nalp6 } & Aldh8a1 & \\
\hline & & 1 \\
\hline & & 0.73200 \\
\hline \multirow[t]{3}{*}{ Nalp6 } & Acat2 & \\
\hline & & 1 \\
\hline & & 0.86328 \\
\hline \multirow[t]{3}{*}{ Nalp6 } & Sucnr1 & \\
\hline & & 7 \\
\hline & & 0.81524 \\
\hline \multirow[t]{2}{*}{ Nalp6 } & Lims2 & \\
\hline & & 8 \\
\hline
\end{tabular}




\begin{tabular}{|c|c|c|}
\hline \multirow[t]{2}{*}{ Nalp6 } & Piga & $\begin{array}{c}0.79560 \\
3\end{array}$ \\
\hline & & 0.87400 \\
\hline \multirow[t]{3}{*}{ Nalp6 } & Agxt2I1 & \\
\hline & & 8 \\
\hline & & 0.90120 \\
\hline \multirow[t]{3}{*}{ Nalp6 } & BC048644 & \\
\hline & & 7 \\
\hline & & 0.92692 \\
\hline \multirow[t]{3}{*}{ Nalp6 } & Proz & \\
\hline & & 1 \\
\hline & & 0.96081 \\
\hline \multirow[t]{3}{*}{ Nalp6 } & LOC240549 & \\
\hline & & 4 \\
\hline & & 0.74590 \\
\hline \multirow[t]{2}{*}{ Nalp6 } & Pbld & \\
\hline & & 9 \\
\hline \multirow[t]{2}{*}{ Nalp6 } & Ung & 0.86821 \\
\hline & & 0.75360 \\
\hline \multirow[t]{3}{*}{ Nalp6 } & Bdh1 & \\
\hline & & 3 \\
\hline & & 0.91160 \\
\hline \multirow[t]{3}{*}{ Nalp6 } & Tmem25 & \\
\hline & & 1 \\
\hline & & 0.94499 \\
\hline \multirow[t]{3}{*}{ Nalp6 } & Ccdc120 & \\
\hline & & 8 \\
\hline & & 0.80338 \\
\hline \multirow[t]{2}{*}{ Nalp6 } & Dhcr7 & \\
\hline & & 3 \\
\hline
\end{tabular}




\begin{tabular}{|c|c|c|}
\hline \multirow[t]{2}{*}{ Nalp6 } & Gpr146 & $\begin{array}{c}0.79344 \\
8\end{array}$ \\
\hline & & 0.77439 \\
\hline \multirow[t]{3}{*}{ Nalp6 } & Asns & \\
\hline & & 1 \\
\hline & & 0.84708 \\
\hline \multirow[t]{3}{*}{ Nalp6 } & Abca8a & \\
\hline & & 5 \\
\hline & & 0.72878 \\
\hline \multirow[t]{3}{*}{ Nalp6 } & Hpgd & \\
\hline & & 8 \\
\hline & & 0.96238 \\
\hline \multirow[t]{3}{*}{ Nalp6 } & Mrpl49 & \\
\hline & & 2 \\
\hline & & 0.81256 \\
\hline \multirow[t]{3}{*}{ Nalp6 } & Slc25a42 & \\
\hline & & 6 \\
\hline & & 0.97987 \\
\hline \multirow[t]{3}{*}{ Nalp6 } & Hsd3b5 & \\
\hline & & 2 \\
\hline & & 0.86922 \\
\hline \multirow[t]{3}{*}{ Nalp6 } & Aox3 & \\
\hline & & 9 \\
\hline & & 0.78881 \\
\hline \multirow[t]{3}{*}{ Nalp6 } & Smox & \\
\hline & & 4 \\
\hline & & 0.79181 \\
\hline \multirow[t]{2}{*}{ Nalp6 } & LOC433886 & \\
\hline & & 4 \\
\hline
\end{tabular}




\begin{tabular}{|c|c|c|}
\hline Nalp6 & Fn3k & $\begin{array}{c}0.95109 \\
4\end{array}$ \\
\hline Nalp6 & Ngef & $\begin{array}{c}0.94850 \\
3\end{array}$ \\
\hline Nalp6 & Zfp750 & $\begin{array}{c}0.98417 \\
6\end{array}$ \\
\hline Nalp6 & Slc41a3 & $\begin{array}{c}0.79970 \\
7\end{array}$ \\
\hline Nalp6 & Hacl1 & $\begin{array}{c}0.79105 \\
9\end{array}$ \\
\hline Nalp6 & Fam129a & 0.96364 \\
\hline Nalp6 & B430005K18Rik & $\begin{array}{c}0.88533 \\
3\end{array}$ \\
\hline Nalp6 & Gas7 & $\begin{array}{c}0.71217 \\
3\end{array}$ \\
\hline Nalp6 & Ces1 & $\begin{array}{c}0.97421 \\
7\end{array}$ \\
\hline Nalp6 & Bves & 0.72402 \\
\hline Nalp6 & Bcdo2 & $\begin{array}{c}0.81543 \\
8\end{array}$ \\
\hline
\end{tabular}




\begin{tabular}{|c|c|c|}
\hline \multirow[t]{2}{*}{ Nalp6 } & Rgs12 & $\begin{array}{c}0.82654 \\
6\end{array}$ \\
\hline & & 0.77632 \\
\hline \multirow[t]{3}{*}{ Nalp6 } & 5730410E15Rik & \\
\hline & & 8 \\
\hline & & 0.70106 \\
\hline \multirow[t]{3}{*}{ Nalp6 } & E030049G20Rik & \\
\hline & & 2 \\
\hline & & 0.80387 \\
\hline \multirow[t]{3}{*}{ Nalp6 } & Gpr152 & \\
\hline & & 8 \\
\hline & & 0.71451 \\
\hline \multirow[t]{3}{*}{ Tlcd2 } & Aldh8a1 & \\
\hline & & 6 \\
\hline & & 0.86272 \\
\hline \multirow[t]{3}{*}{ Tlcd2 } & Acat2 & \\
\hline & & 1 \\
\hline & & 0.92990 \\
\hline \multirow[t]{3}{*}{ Tlcd2 } & Sucnr1 & \\
\hline & & 3 \\
\hline & & 0.82106 \\
\hline \multirow[t]{3}{*}{ Tlcd2 } & Lims2 & \\
\hline & & 9 \\
\hline & & 0.85250 \\
\hline \multirow[t]{2}{*}{ Tlcd2 } & Piga & \\
\hline & & 8 \\
\hline \multirow[t]{2}{*}{ Tlcd2 } & 1700019H03Rik & 0.76488 \\
\hline & & 0.72721 \\
\hline \multirow[t]{2}{*}{ Tlcd2 } & Agxt2I1 & \\
\hline & & 8 \\
\hline
\end{tabular}




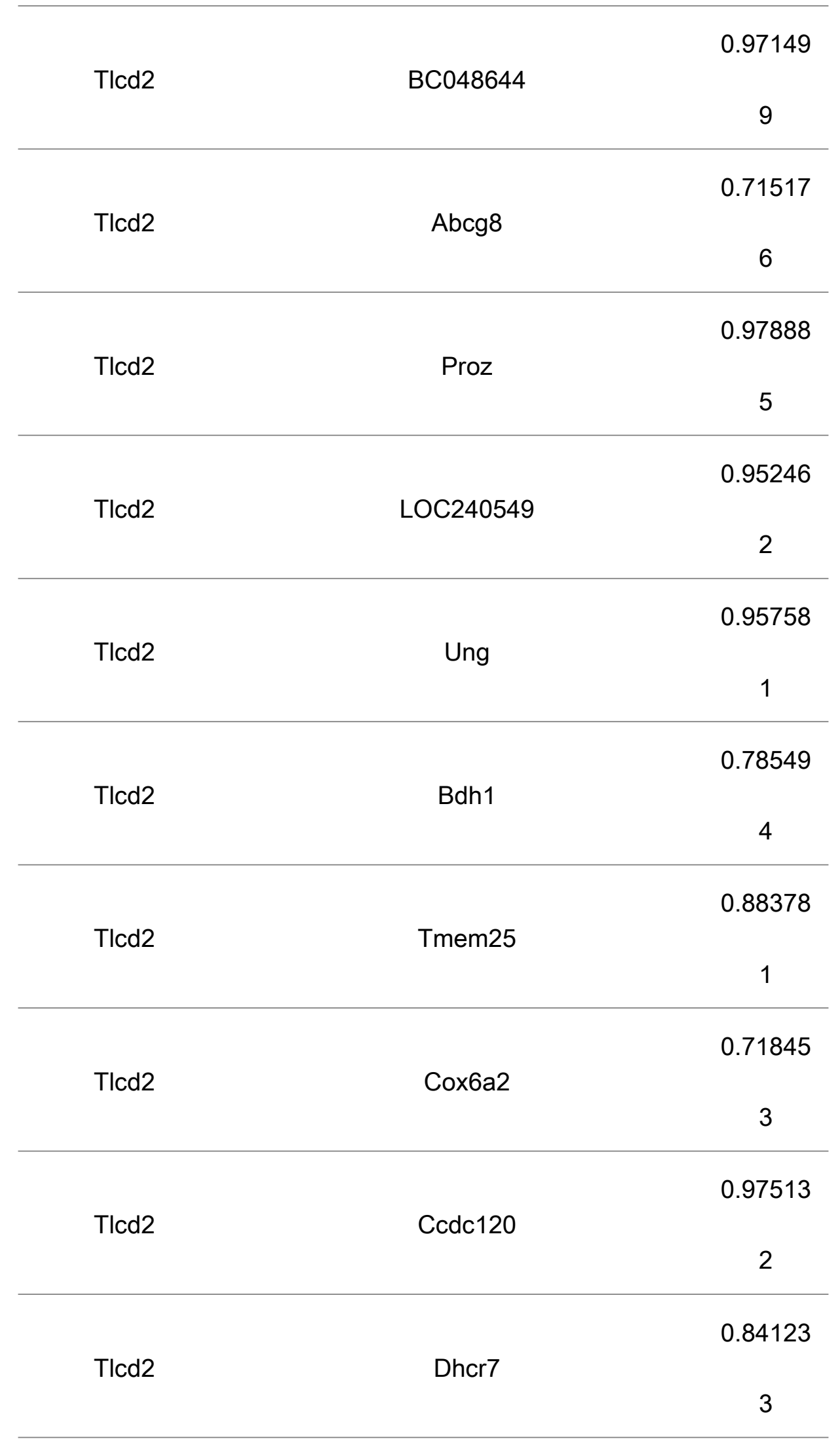




\begin{tabular}{|c|c|c|}
\hline \multirow[t]{2}{*}{ Tlcd2 } & Mist1 & $\begin{array}{c}0.70283 \\
2\end{array}$ \\
\hline & & 0.86939 \\
\hline \multirow[t]{3}{*}{ Tlcd2 } & Gpr146 & \\
\hline & & 6 \\
\hline & & 0.83530 \\
\hline \multirow[t]{3}{*}{ Tlcd2 } & Asns & \\
\hline & & 3 \\
\hline & & 0.80889 \\
\hline \multirow[t]{3}{*}{ Tlcd2 } & Abca8a & \\
\hline & & 9 \\
\hline & & 0.78321 \\
\hline \multirow[t]{3}{*}{ Tlcd2 } & Hpgd & \\
\hline & & 8 \\
\hline & & 0.85457 \\
\hline \multirow[t]{3}{*}{ Tlcd2 } & Mrpl49 & \\
\hline & & 1 \\
\hline & & 0.92612 \\
\hline \multirow[t]{3}{*}{ Tlcd2 } & Slc25a42 & \\
\hline & & 4 \\
\hline & & 0.80109 \\
\hline \multirow[t]{3}{*}{ Tlcd2 } & Pmm1 & \\
\hline & & 2 \\
\hline & & 0.93444 \\
\hline \multirow[t]{3}{*}{ Tlcd2 } & Hsd3b5 & \\
\hline & & 6 \\
\hline & & 0.87328 \\
\hline \multirow[t]{2}{*}{ Tlcd2 } & Aox3 & \\
\hline & & 5 \\
\hline
\end{tabular}




\begin{tabular}{|c|c|c|}
\hline \multirow[t]{2}{*}{ Tlcd2 } & Smox & $\begin{array}{c}0.87741 \\
9\end{array}$ \\
\hline & & 0.86863 \\
\hline \multirow[t]{3}{*}{ Tlcd2 } & LOC433886 & \\
\hline & & 1 \\
\hline & & 0.82833 \\
\hline \multirow[t]{3}{*}{ Tlcd2 } & Fn3k & \\
\hline & & 8 \\
\hline & & 0.95708 \\
\hline \multirow[t]{2}{*}{ Tlcd2 } & Ngef & \\
\hline & & 3 \\
\hline \multirow[t]{2}{*}{ Tlcd2 } & Zfp750 & 0.88953 \\
\hline & & 0.75126 \\
\hline \multirow[t]{3}{*}{ Tlcd2 } & 1810020C02Rik & \\
\hline & & 5 \\
\hline & & 0.89943 \\
\hline \multirow[t]{3}{*}{ Tlcd2 } & Slc41a3 & \\
\hline & & 4 \\
\hline & & 0.78167 \\
\hline \multirow[t]{3}{*}{ Tlcd2 } & Sel1h & \\
\hline & & 7 \\
\hline & & 0.85774 \\
\hline \multirow[t]{2}{*}{ Tlcd2 } & Fam129a & \\
\hline & & 7 \\
\hline \multirow[t]{2}{*}{ Tlcd2 } & Ica1 & 0.73496 \\
\hline & & 0.70699 \\
\hline \multirow[t]{2}{*}{ Tlcd2 } & 4432416J03Rik & \\
\hline & & 9 \\
\hline
\end{tabular}




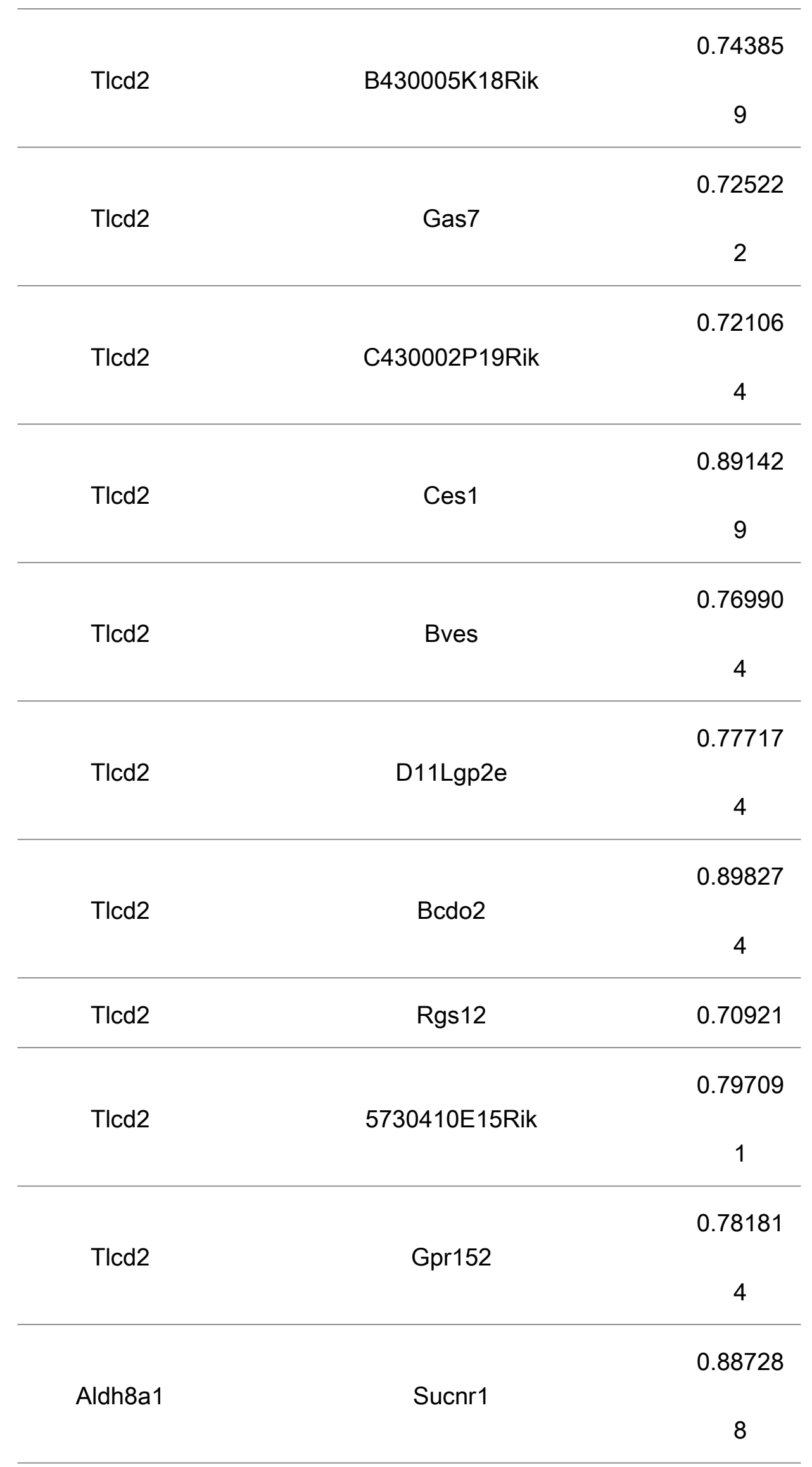




\begin{tabular}{|c|c|c|}
\hline Aldh8a1 & Lims2 & 0.97835 \\
\hline \multirow[t]{2}{*}{ Aldh8a1 } & Proz & 0.81412 \\
\hline & & 0.90222 \\
\hline \multirow[t]{3}{*}{ Aldh8a1 } & Apoa5 & \\
\hline & & 8 \\
\hline & & 0.73045 \\
\hline \multirow[t]{3}{*}{ Aldh8a1 } & LOC240549 & \\
\hline & & 3 \\
\hline & & 0.71359 \\
\hline \multirow[t]{3}{*}{ Aldh8a1 } & LOC380998 & \\
\hline & & 1 \\
\hline & & 0.79404 \\
\hline \multirow[t]{3}{*}{ Aldh8a1 } & Pbld & \\
\hline & & 9 \\
\hline & & 0.77465 \\
\hline \multirow[t]{3}{*}{ Aldh8a1 } & Ung & \\
\hline & & 2 \\
\hline & & 0.98489 \\
\hline \multirow[t]{3}{*}{ Aldh8a1 } & Bdh1 & \\
\hline & & 5 \\
\hline & & 0.87671 \\
\hline \multirow[t]{3}{*}{ Aldh8a1 } & Tmem25 & \\
\hline & & 7 \\
\hline & & 0.72153 \\
\hline \multirow[t]{3}{*}{ Aldh8a1 } & Ccdc120 & \\
\hline & & 4 \\
\hline & & 0.96066 \\
\hline \multirow[t]{2}{*}{ Aldh8a1 } & Dhcr7 & \\
\hline & & 4 \\
\hline
\end{tabular}




\begin{tabular}{|c|c|c|}
\hline \multirow[t]{2}{*}{ Aldh8a1 } & Asns & $\begin{array}{c}0.80446 \\
2\end{array}$ \\
\hline & & 0.93652 \\
\hline \multirow[t]{2}{*}{ Aldh8a1 } & Abca8a & \\
\hline & & 9 \\
\hline \multirow[t]{2}{*}{ Aldh8a1 } & Mrpl49 & 0.73807 \\
\hline & & 0.91991 \\
\hline \multirow[t]{3}{*}{ Aldh8a1 } & 3010026009Rik & \\
\hline & & 9 \\
\hline & & 0.81493 \\
\hline \multirow[t]{3}{*}{ Aldh8a1 } & Paqr7 & \\
\hline & & 3 \\
\hline & & 0.88718 \\
\hline \multirow[t]{2}{*}{ Aldh8a1 } & Aox3 & \\
\hline & & 1 \\
\hline \multirow[t]{2}{*}{ Aldh8a1 } & LOC100047427 & 0.72582 \\
\hline & & 0.76946 \\
\hline \multirow[t]{2}{*}{ Aldh8a1 } & LOC433886 & \\
\hline & & 4 \\
\hline \multirow[t]{2}{*}{ Aldh8a1 } & Ngef & 0.80509 \\
\hline & & 0.78370 \\
\hline \multirow[t]{3}{*}{ Aldh8a1 } & Prss8 & \\
\hline & & 7 \\
\hline & & 0.70806 \\
\hline \multirow[t]{3}{*}{ Aldh8a1 } & Fam129a & \\
\hline & & 9 \\
\hline & & 0.73630 \\
\hline \multirow[t]{2}{*}{ Aldh8a1 } & Ica1 & \\
\hline & & 8 \\
\hline
\end{tabular}




\begin{tabular}{|c|c|c|}
\hline Aldh8a1 & Cabc1 & 0.7476 \\
\hline & & 0.74454 \\
\hline \multirow[t]{3}{*}{ Aldh8a1 } & 4432416J03Rik & \\
\hline & & 2 \\
\hline & & 0.90997 \\
\hline \multirow[t]{3}{*}{ Aldh8a1 } & Slc27a3 & \\
\hline & & 1 \\
\hline & & 0.71818 \\
\hline \multirow[t]{3}{*}{ Aldh8a1 } & Gins2 & \\
\hline & & 5 \\
\hline & & 0.85333 \\
\hline \multirow[t]{3}{*}{ Aldh8a1 } & D11Lgp2e & \\
\hline & & 3 \\
\hline & & 0.91809 \\
\hline \multirow[t]{3}{*}{ Aldh8a1 } & Zfp57 & \\
\hline & & 3 \\
\hline & & 0.70304 \\
\hline \multirow[t]{3}{*}{ Aldh8a1 } & D930009C14Rik & \\
\hline & & 3 \\
\hline & & 0.84212 \\
\hline \multirow[t]{3}{*}{ Aldh8a1 } & Bcdo2 & \\
\hline & & 3 \\
\hline & & 0.73696 \\
\hline \multirow[t]{3}{*}{ Aldh8a1 } & 2610042O14Rik & \\
\hline & & 7 \\
\hline & & 0.79468 \\
\hline \multirow[t]{3}{*}{ Aldh8a1 } & 5730410E15Rik & \\
\hline & & 2 \\
\hline & & 0.73491 \\
\hline \multirow[t]{2}{*}{ Aldh8a1 } & E030049G20Rik & \\
\hline & & 5 \\
\hline
\end{tabular}




\begin{tabular}{|c|c|c|}
\hline \multirow[t]{2}{*}{ Aldh8a1 } & Mpdz & $\begin{array}{c}0.70667 \\
1\end{array}$ \\
\hline & & 0.83153 \\
\hline \multirow[t]{3}{*}{ Cldn2 } & Acat2 & \\
\hline & & 2 \\
\hline & & 0.74704 \\
\hline \multirow[t]{3}{*}{ Cldn2 } & Sucnr1 & \\
\hline & & 3 \\
\hline & & 0.93861 \\
\hline \multirow[t]{3}{*}{ Cldn2 } & 1110046J11Rik & \\
\hline & & 7 \\
\hline & & 0.90267 \\
\hline \multirow[t]{3}{*}{ Cldn2 } & Abcg8 & \\
\hline & & 7 \\
\hline & & 0.86826 \\
\hline \multirow[t]{3}{*}{ Cldn2 } & Apoa5 & \\
\hline & & 1 \\
\hline & & 0.91541 \\
\hline \multirow[t]{3}{*}{ Cldn2 } & C730029A08Rik & \\
\hline & & 5 \\
\hline & & 0.72965 \\
\hline \multirow[t]{2}{*}{ Cldn2 } & LOC380998 & \\
\hline & & 1 \\
\hline \multirow[t]{2}{*}{ Cldn2 } & Ung & 0.75075 \\
\hline & & 0.72215 \\
\hline \multirow[t]{3}{*}{ Cldn2 } & Bdh1 & \\
\hline & & 3 \\
\hline & & 0.73608 \\
\hline \multirow[t]{2}{*}{ Cldn2 } & Dhcr7 & \\
\hline & & 3 \\
\hline
\end{tabular}




\begin{tabular}{|c|c|c|}
\hline \multirow[t]{2}{*}{ Cldn2 } & Asns & $\begin{array}{c}0.76101 \\
5\end{array}$ \\
\hline & & 0.74882 \\
\hline \multirow[t]{3}{*}{ Cldn2 } & 3010026009Rik & \\
\hline & & 6 \\
\hline & & 0.70525 \\
\hline \multirow[t]{3}{*}{ Cldn2 } & LOC433886 & \\
\hline & & 6 \\
\hline & & 0.96170 \\
\hline \multirow[t]{2}{*}{ Cldn2 } & Pdzk1ip1 & \\
\hline & & 7 \\
\hline Cldn2 & Slc41a3 & 0.72827 \\
\hline \multirow[t]{2}{*}{ Cldn2 } & Ica1 & 0.74737 \\
\hline & & 0.74055 \\
\hline \multirow[t]{3}{*}{ Cldn2 } & Map3k6 & \\
\hline & & 8 \\
\hline & & 0.97618 \\
\hline \multirow[t]{3}{*}{ Cldn2 } & 4432416J03Rik & \\
\hline & & 3 \\
\hline & & 0.78848 \\
\hline \multirow[t]{3}{*}{ Cldn2 } & D930015E06Rik & \\
\hline & & 9 \\
\hline & & 0.82415 \\
\hline \multirow[t]{3}{*}{ Cldn2 } & C430002P19Rik & \\
\hline & & 3 \\
\hline & & 0.70106 \\
\hline \multirow[t]{2}{*}{ Cldn2 } & Gins2 & \\
\hline & & 3 \\
\hline
\end{tabular}




\begin{tabular}{|c|c|c|}
\hline \multirow[t]{2}{*}{ Cldn2 } & D11Lgp2e & $\begin{array}{c}0.90429 \\
9\end{array}$ \\
\hline & & 0.76560 \\
\hline \multirow[t]{3}{*}{ Cldn2 } & Ube2u & \\
\hline & & 5 \\
\hline & & 0.71203 \\
\hline \multirow[t]{2}{*}{ Cldn2 } & Bcdo2 & \\
\hline & & 4 \\
\hline \multirow[t]{2}{*}{ Cldn2 } & Cnksr1 & 0.84073 \\
\hline & & 0.77595 \\
\hline \multirow[t]{2}{*}{ Cldn2 } & 2610042O14Rik & \\
\hline & & 1 \\
\hline \multirow[t]{2}{*}{ Cldn2 } & B230207L18Rik & 0.89822 \\
\hline & & 0.87828 \\
\hline \multirow[t]{2}{*}{ Acat2 } & Sucnr1 & \\
\hline & & 1 \\
\hline \multirow[t]{2}{*}{ Acat2 } & Lims2 & 0.77059 \\
\hline & & 0.81286 \\
\hline \multirow[t]{3}{*}{ Acat2 } & Piga & \\
\hline & & 3 \\
\hline & & 0.73132 \\
\hline \multirow[t]{3}{*}{ Acat2 } & 1700019H03Rik & \\
\hline & & 3 \\
\hline & & 0.81070 \\
\hline \multirow[t]{3}{*}{ Acat2 } & BC048644 & \\
\hline & & 1 \\
\hline & & 0.88790 \\
\hline \multirow[t]{2}{*}{ Acat2 } & Abcg8 & \\
\hline & & 1 \\
\hline
\end{tabular}




\begin{tabular}{|c|c|c|}
\hline \multirow[t]{2}{*}{ Acat2 } & Proz & $\begin{array}{c}0.90440 \\
7\end{array}$ \\
\hline & & 0.76288 \\
\hline \multirow[t]{3}{*}{ Acat2 } & Apoa5 & \\
\hline & & 5 \\
\hline & & 0.77510 \\
\hline \multirow[t]{3}{*}{ Acat2 } & LOC240549 & \\
\hline & & 8 \\
\hline & & 0.94093 \\
\hline \multirow[t]{3}{*}{ Acat2 } & Ung & \\
\hline & & 8 \\
\hline & & 0.74826 \\
\hline \multirow[t]{3}{*}{ Acat2 } & Bdh1 & \\
\hline & & 7 \\
\hline & & 0.70980 \\
\hline \multirow[t]{3}{*}{ Acat2 } & Tmem25 & \\
\hline & & 1 \\
\hline & & 0.84025 \\
\hline \multirow[t]{3}{*}{ Acat2 } & Ccdc120 & \\
\hline & & 9 \\
\hline & & 0.81926 \\
\hline \multirow[t]{3}{*}{ Acat2 } & Dhcr7 & \\
\hline & & 3 \\
\hline & & 0.71374 \\
\hline \multirow[t]{3}{*}{ Acat2 } & Gpr146 & \\
\hline & & 2 \\
\hline & & 0.93549 \\
\hline \multirow[t]{2}{*}{ Acat2 } & Asns & \\
\hline & & 6 \\
\hline
\end{tabular}




\begin{tabular}{|c|c|c|}
\hline \multirow[t]{2}{*}{ Acat2 } & Hpgd & $\begin{array}{c}0.88926 \\
4\end{array}$ \\
\hline & & 0.79986 \\
\hline \multirow[t]{3}{*}{ Acat2 } & Slc25a42 & \\
\hline & & 4 \\
\hline & & 0.72596 \\
\hline \multirow[t]{3}{*}{ Acat2 } & Pmm1 & \\
\hline & & 4 \\
\hline & & 0.70399 \\
\hline \multirow[t]{3}{*}{ Acat2 } & Hsd3b5 & \\
\hline & & 7 \\
\hline & & 0.73154 \\
\hline \multirow[t]{3}{*}{ Acat2 } & Aox3 & \\
\hline & & 3 \\
\hline & & 0.71952 \\
\hline \multirow[t]{3}{*}{ Acat2 } & Smox & \\
\hline & & 2 \\
\hline & & 0.80926 \\
\hline \multirow[t]{3}{*}{ Acat2 } & LOC433886 & \\
\hline & & 3 \\
\hline & & 0.86606 \\
\hline \multirow[t]{3}{*}{ Acat2 } & Ngef & \\
\hline & & 7 \\
\hline & & 0.82852 \\
\hline \multirow[t]{3}{*}{ Acat2 } & Pdzk1ip1 & \\
\hline & & 4 \\
\hline & & 0.92132 \\
\hline \multirow[t]{2}{*}{ Acat2 } & Slc41a3 & \\
\hline & & 2 \\
\hline
\end{tabular}




\begin{tabular}{|c|c|c|}
\hline \multirow[t]{2}{*}{ Acat2 } & Sel1h & $\begin{array}{c}0.91298 \\
3\end{array}$ \\
\hline & & 0.89619 \\
\hline \multirow[t]{3}{*}{ Acat2 } & Ica1 & \\
\hline & & 5 \\
\hline & & 0.71388 \\
\hline \multirow[t]{3}{*}{ Acat2 } & Map3k6 & \\
\hline & & 6 \\
\hline & & 0.91502 \\
\hline \multirow[t]{3}{*}{ Acat2 } & 4432416J03Rik & \\
\hline & & 5 \\
\hline & & 0.78091 \\
\hline \multirow[t]{3}{*}{ Acat2 } & LOC242997 & \\
\hline & & 4 \\
\hline & & 0.73938 \\
\hline \multirow[t]{3}{*}{ Acat2 } & Slc27a3 & \\
\hline & & 7 \\
\hline & & 0.87641 \\
\hline \multirow[t]{3}{*}{ Acat2 } & C430002P19Rik & \\
\hline & & 4 \\
\hline & & 0.72267 \\
\hline \multirow[t]{3}{*}{ Acat2 } & Gins2 & \\
\hline & & 3 \\
\hline & & 0.85840 \\
\hline \multirow[t]{3}{*}{ Acat2 } & D11Lgp2e & \\
\hline & & 7 \\
\hline & & 0.80172 \\
\hline \multirow[t]{2}{*}{ Acat2 } & Bcdo2 & \\
\hline & & 9 \\
\hline
\end{tabular}




\begin{tabular}{|c|c|c|}
\hline \multirow[t]{2}{*}{ Acat2 } & 5730410E15Rik & $\begin{array}{c}0.70330 \\
2\end{array}$ \\
\hline & & 0.80445 \\
\hline \multirow[t]{3}{*}{ Acat2 } & B230207L18Rik & \\
\hline & & 6 \\
\hline & & 0.94656 \\
\hline \multirow[t]{2}{*}{ Sucnr1 } & Lims2 & \\
\hline & & 1 \\
\hline \multirow[t]{2}{*}{ Sucnr1 } & Piga & 0.79759 \\
\hline & & 0.78451 \\
\hline \multirow[t]{3}{*}{ Sucnr1 } & 1700019H03Rik & \\
\hline & & 4 \\
\hline & & 0.84032 \\
\hline \multirow[t]{3}{*}{ Sucnr1 } & BC048644 & \\
\hline & & 5 \\
\hline & & 0.79069 \\
\hline \multirow[t]{3}{*}{ Sucnr1 } & Abcg8 & \\
\hline & & 5 \\
\hline & & 0.97730 \\
\hline \multirow[t]{3}{*}{ Sucnr1 } & Proz & \\
\hline & & 7 \\
\hline & & 0.88325 \\
\hline \multirow[t]{3}{*}{ Sucnr1 } & Apoa5 & \\
\hline & & 7 \\
\hline & & 0.88221 \\
\hline \multirow[t]{3}{*}{ Sucnr1 } & LOC240549 & \\
\hline & & 1 \\
\hline & & 0.95539 \\
\hline \multirow[t]{2}{*}{ Sucnr1 } & Ung & \\
\hline & & 5 \\
\hline
\end{tabular}




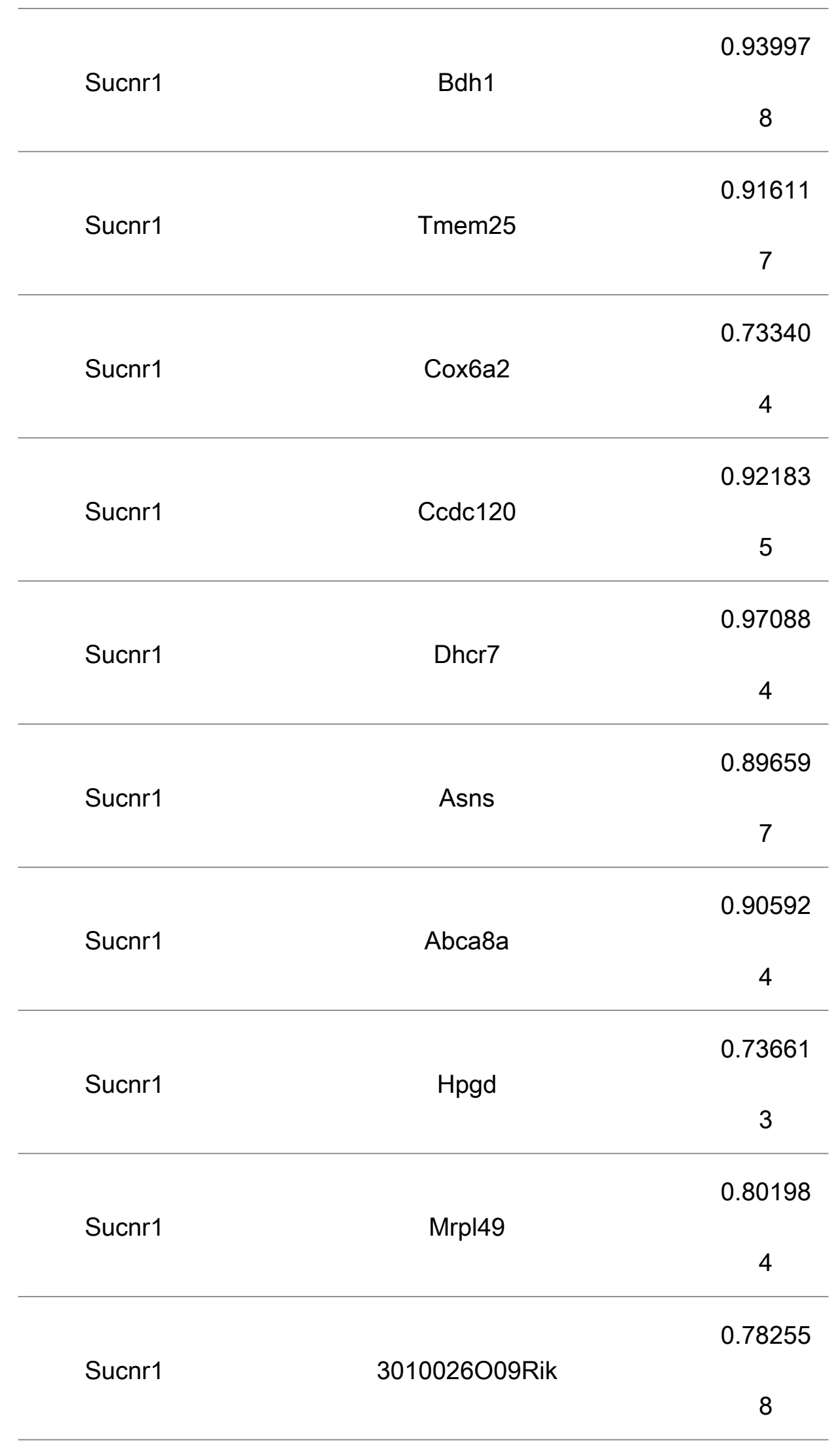




\begin{tabular}{|c|c|c|}
\hline Sucnr1 & Slc25a42 & $\begin{array}{c}0.83178 \\
7\end{array}$ \\
\hline Sucnr1 & Pmm1 & 0.80431 \\
\hline Sucnr1 & Hsd3b5 & $\begin{array}{c}0.78753 \\
8\end{array}$ \\
\hline Sucnr1 & Aox3 & $\begin{array}{c}0.94743 \\
4\end{array}$ \\
\hline Sucnr1 & Smox & $\begin{array}{c}0.88498 \\
1\end{array}$ \\
\hline Sucnr1 & LOC433886 & $\begin{array}{c}0.89318 \\
2\end{array}$ \\
\hline Sucnr1 & Fn3k & $\begin{array}{c}0.74823 \\
7\end{array}$ \\
\hline Sucnr1 & Ngef & $\begin{array}{c}0.93512 \\
2\end{array}$ \\
\hline Sucnr1 & Zfp750 & $\begin{array}{c}0.80580 \\
2\end{array}$ \\
\hline Sucnr1 & 1810020C02Rik & $\begin{array}{c}0.74367 \\
7\end{array}$ \\
\hline Sucnr1 & Pdzk1ip1 & $\begin{array}{c}0.70123 \\
3\end{array}$ \\
\hline
\end{tabular}




\begin{tabular}{|c|c|c|}
\hline Sucnr1 & Slc41a3 & 0.86666 \\
\hline & & 0.72524 \\
\hline \multirow[t]{3}{*}{ Sucnr1 } & Sel1h & \\
\hline & & 9 \\
\hline & & 0.79518 \\
\hline \multirow[t]{3}{*}{ Sucnr1 } & Fam129a & \\
\hline & & 2 \\
\hline & & 0.79661 \\
\hline \multirow[t]{3}{*}{ Sucnr1 } & Ica1 & \\
\hline & & 2 \\
\hline & & 0.83897 \\
\hline \multirow[t]{3}{*}{ Sucnr1 } & 4432416J03Rik & \\
\hline & & 5 \\
\hline & & 0.81627 \\
\hline \multirow[t]{3}{*}{ Sucnr1 } & Slc27a3 & \\
\hline & & 2 \\
\hline & & 0.72805 \\
\hline \multirow[t]{2}{*}{ Sucnr1 } & C430002P19Rik & \\
\hline & & 1 \\
\hline \multirow[t]{2}{*}{ Sucnr1 } & Ces1 & 0.77008 \\
\hline & & 0.71037 \\
\hline \multirow[t]{3}{*}{ Sucnr1 } & Gins2 & \\
\hline & & 6 \\
\hline & & 0.72187 \\
\hline \multirow[t]{3}{*}{ Sucnr1 } & Bves & \\
\hline & & 5 \\
\hline & & 0.91604 \\
\hline \multirow[t]{2}{*}{ Sucnr1 } & D11Lgp2e & \\
\hline & & 1 \\
\hline Sucnr1 & Zfp57 & 0.71914 \\
\hline
\end{tabular}




\begin{tabular}{|c|c|c|}
\hline \multirow[t]{2}{*}{ Sucnr1 } & Bcdo2 & $\begin{array}{c}0.97225 \\
8\end{array}$ \\
\hline & & 0.87527 \\
\hline \multirow[t]{3}{*}{ Sucnr1 } & 5730410E15Rik & \\
\hline & & 8 \\
\hline & & 0.72355 \\
\hline \multirow[t]{3}{*}{ Sucnr1 } & E030049G20Rik & \\
\hline & & 6 \\
\hline & & 0.71461 \\
\hline \multirow[t]{3}{*}{ Sucnr1 } & Zfp800 & \\
\hline & & 8 \\
\hline & & 0.71902 \\
\hline \multirow[t]{3}{*}{ Sucnr1 } & Gpr152 & \\
\hline & & 9 \\
\hline & & 0.72496 \\
\hline \multirow[t]{3}{*}{ Lims2 } & Piga & \\
\hline & & 4 \\
\hline & & 0.90511 \\
\hline \multirow[t]{3}{*}{ Lims2 } & Proz & \\
\hline & & 2 \\
\hline & & 0.88103 \\
\hline \multirow[t]{3}{*}{ Lims2 } & Apoa5 & \\
\hline & & 6 \\
\hline & & 0.82568 \\
\hline \multirow[t]{3}{*}{ Lims2 } & LOC240549 & \\
\hline & & 4 \\
\hline & & 0.80227 \\
\hline \multirow[t]{2}{*}{ Lims2 } & Pbld & \\
\hline & & 5 \\
\hline
\end{tabular}




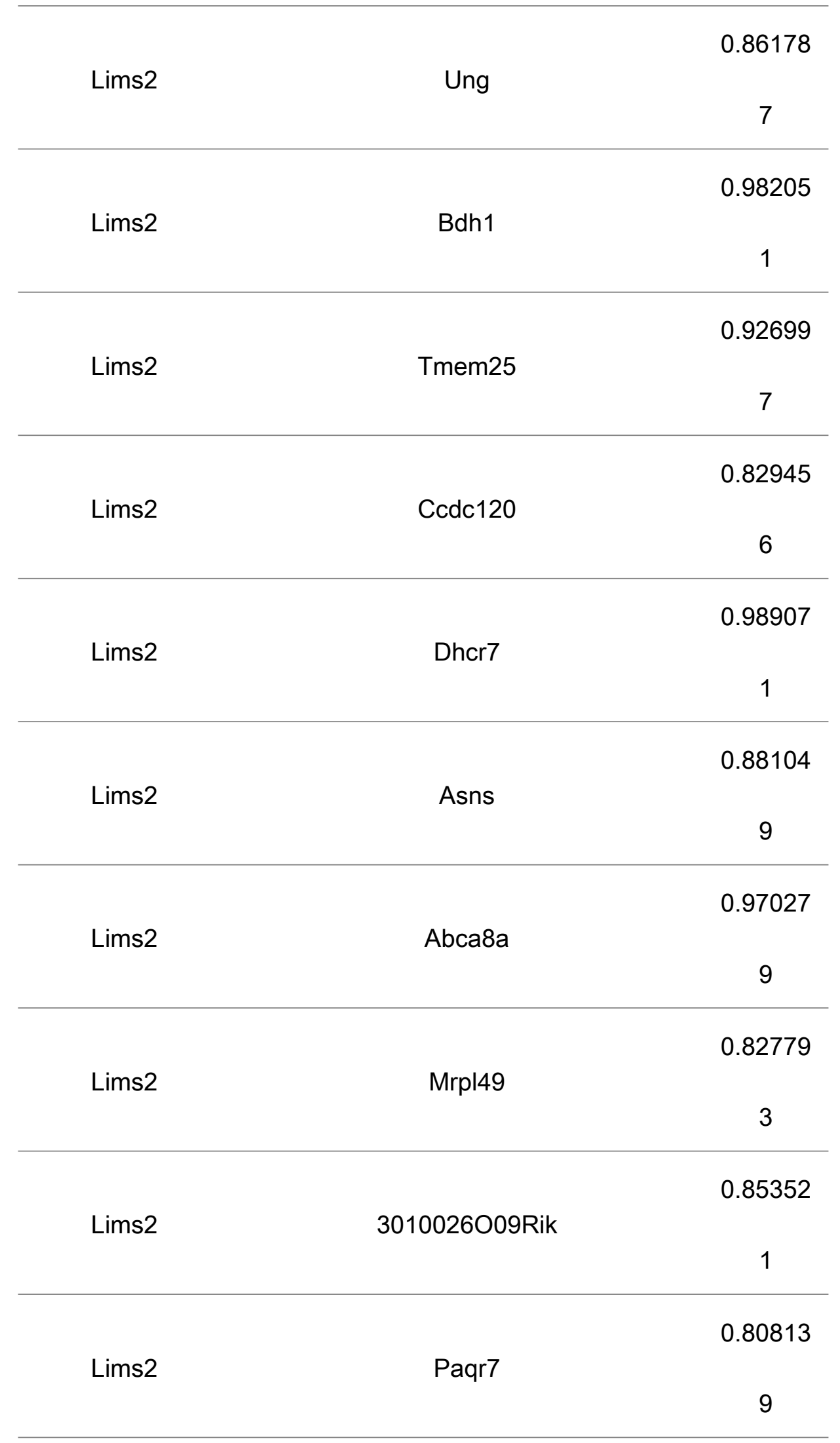




\begin{tabular}{|c|c|c|}
\hline Lims2 & Hsd3b5 & $\begin{array}{c}0.71029 \\
4\end{array}$ \\
\hline Lims2 & Aox3 & $\begin{array}{c}0.93635 \\
3\end{array}$ \\
\hline Lims2 & Smox & $\begin{array}{c}0.74898 \\
6\end{array}$ \\
\hline Lims2 & LOC433886 & $\begin{array}{c}0.82301 \\
5\end{array}$ \\
\hline Lims2 & Fn3k & $\begin{array}{c}0.76497 \\
2\end{array}$ \\
\hline Lims2 & Ngef & $\begin{array}{c}0.90009 \\
1\end{array}$ \\
\hline Lims2 & Zfp750 & $\begin{array}{c}0.79758 \\
9\end{array}$ \\
\hline Lims2 & Slc41a3 & $\begin{array}{c}0.76552 \\
8\end{array}$ \\
\hline Lims2 & Fam129a & $\begin{array}{c}0.80538 \\
5\end{array}$ \\
\hline Lims2 & Ica1 & $\begin{array}{c}0.80421 \\
2\end{array}$ \\
\hline
\end{tabular}




\begin{tabular}{|c|c|c|}
\hline \multirow[t]{2}{*}{ Lims2 } & 4432416J03Rik & $\begin{array}{c}0.77577 \\
3\end{array}$ \\
\hline & & 0.92155 \\
\hline \multirow[t]{2}{*}{ Lims2 } & Slc27a3 & \\
\hline & & 6 \\
\hline \multirow[t]{2}{*}{ Lims2 } & Ces1 & 0.72848 \\
\hline & & 0.73854 \\
\hline \multirow[t]{3}{*}{ Lims2 } & Gins2 & \\
\hline & & 1 \\
\hline & & 0.86872 \\
\hline \multirow[t]{2}{*}{ Lims2 } & D11Lgp2e & \\
\hline & & 9 \\
\hline \multirow[t]{2}{*}{ Lims2 } & Zfp57 & 0.84595 \\
\hline & & 0.76417 \\
\hline \multirow[t]{3}{*}{ Lims2 } & D930009C14Rik & \\
\hline & & 8 \\
\hline & & 0.88814 \\
\hline \multirow[t]{3}{*}{ Lims2 } & Bcdo2 & \\
\hline & & 5 \\
\hline & & 0.71197 \\
\hline \multirow[t]{3}{*}{ Lims2 } & 2610042O14Rik & \\
\hline & & 6 \\
\hline & & 0.85003 \\
\hline \multirow[t]{3}{*}{ Lims2 } & 5730410E15Rik & \\
\hline & & 1 \\
\hline & & 0.73645 \\
\hline \multirow[t]{2}{*}{ Lims2 } & E030049G20Rik & \\
\hline & & 8 \\
\hline Dnajb9 & AW011738 & 0.75519 \\
\hline
\end{tabular}




\begin{tabular}{|c|c|c|}
\hline \multirow[t]{2}{*}{ Dnajb9 } & Hspa5 & $\begin{array}{c}0.71666 \\
3\end{array}$ \\
\hline & & 0.81228 \\
\hline \multirow[t]{3}{*}{ Dnajb9 } & Isg20 & \\
\hline & & 6 \\
\hline & & 0.96837 \\
\hline \multirow[t]{3}{*}{ Dnajb9 } & Aoc2 & \\
\hline & & 6 \\
\hline & & 0.72490 \\
\hline \multirow[t]{3}{*}{ Dnajb9 } & Srd5a2 & \\
\hline & & 2 \\
\hline & & 0.79923 \\
\hline \multirow[t]{3}{*}{ Dnajb9 } & 9530048009Rik & \\
\hline & & 2 \\
\hline & & 0.73954 \\
\hline \multirow[t]{3}{*}{ Dnajb9 } & Nfxl1 & \\
\hline & & 2 \\
\hline & & 0.87307 \\
\hline \multirow[t]{3}{*}{ Gmds } & $\mathrm{Hr}$ & \\
\hline & & 3 \\
\hline & & 0.94896 \\
\hline \multirow[t]{3}{*}{ Gmds } & Mthfd2 & \\
\hline & & 2 \\
\hline & & 0.91820 \\
\hline \multirow[t]{3}{*}{ Gmds } & A630034I12Rik & \\
\hline & & 4 \\
\hline & & 0.84122 \\
\hline \multirow[t]{2}{*}{ Gmds } & Niban & \\
\hline & & 1 \\
\hline
\end{tabular}




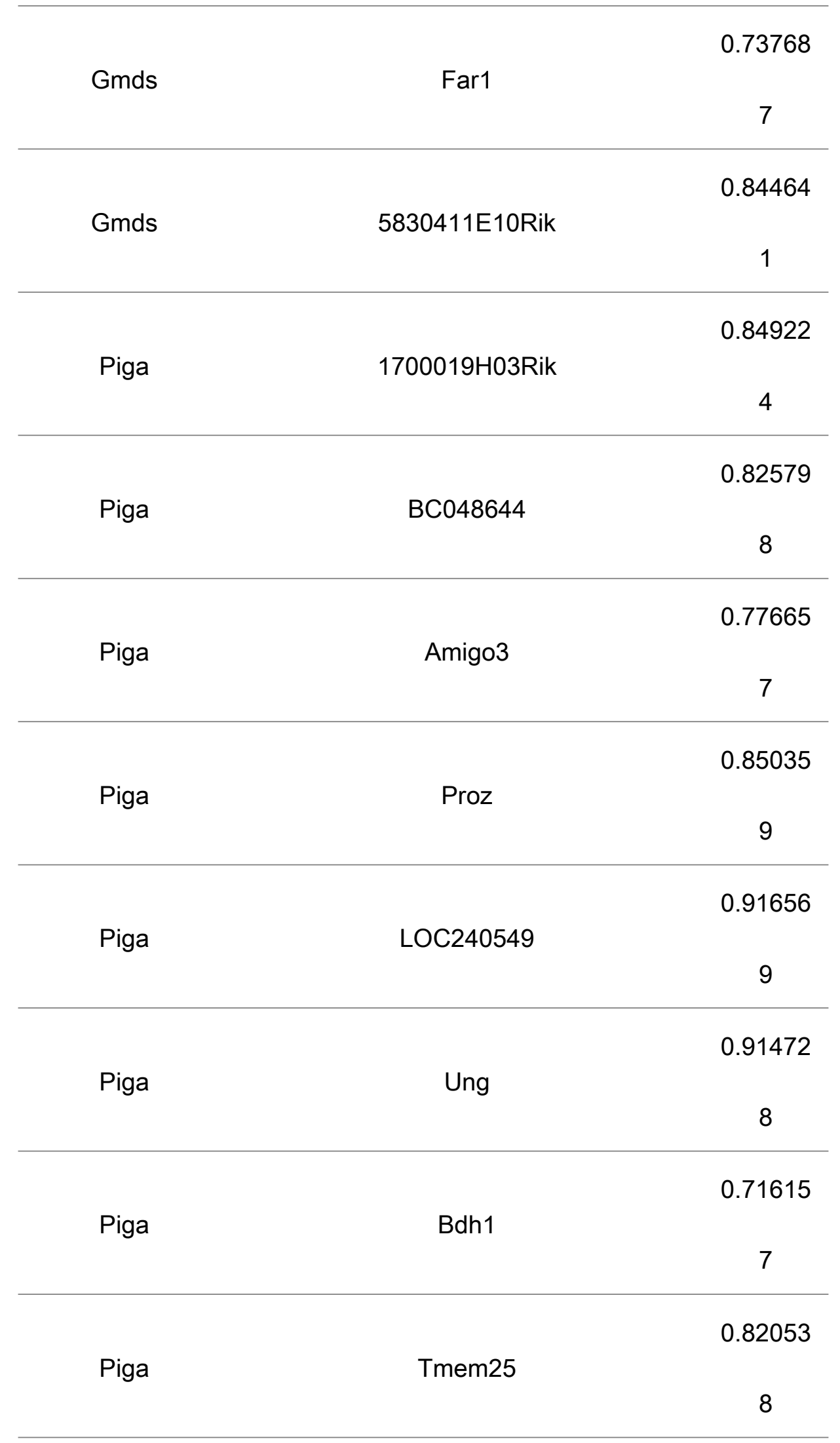




\begin{tabular}{|c|c|c|}
\hline \multirow[t]{2}{*}{ Piga } & Ccdc120 & $\begin{array}{c}0.74773 \\
2\end{array}$ \\
\hline & & 0.71709 \\
\hline \multirow[t]{3}{*}{ Piga } & Dhcr7 & \\
\hline & & 3 \\
\hline & & 0.76848 \\
\hline \multirow[t]{3}{*}{ Piga } & Mist1 & \\
\hline & & 4 \\
\hline & & 0.71845 \\
\hline \multirow[t]{3}{*}{ Piga } & Gpr146 & \\
\hline & & 5 \\
\hline & & 0.76367 \\
\hline \multirow[t]{3}{*}{ Piga } & Asns & \\
\hline & & 1 \\
\hline & & 0.73975 \\
\hline \multirow[t]{3}{*}{ Piga } & Bhlhb8 & \\
\hline & & 8 \\
\hline & & 0.73522 \\
\hline \multirow[t]{3}{*}{ Piga } & Mrpl49 & \\
\hline & & 6 \\
\hline & & 0.84199 \\
\hline \multirow[t]{2}{*}{ Piga } & Slc25a42 & \\
\hline & & 9 \\
\hline \multirow[t]{2}{*}{ Piga } & Hsd3b5 & 0.77341 \\
\hline & & 0.93883 \\
\hline \multirow[t]{3}{*}{ Piga } & LOC433886 & \\
\hline & & 6 \\
\hline & & 0.73115 \\
\hline \multirow[t]{2}{*}{ Piga } & Fn3k & \\
\hline & & 1 \\
\hline
\end{tabular}




\begin{tabular}{|c|c|c|}
\hline \multirow[t]{2}{*}{ Piga } & Ngef & $\begin{array}{c}0.88907 \\
8\end{array}$ \\
\hline & & 0.73569 \\
\hline \multirow[t]{3}{*}{ Piga } & Zfp750 & \\
\hline & & 7 \\
\hline & & 0.96931 \\
\hline \multirow[t]{3}{*}{ Piga } & Slc41a3 & \\
\hline & & 3 \\
\hline & & 0.70194 \\
\hline \multirow[t]{3}{*}{ Piga } & Sel1h & \\
\hline & & 8 \\
\hline & & 0.76526 \\
\hline \multirow[t]{3}{*}{ Piga } & Ica1 & \\
\hline & & 9 \\
\hline & & 0.80717 \\
\hline \multirow[t]{3}{*}{ Piga } & Map3k6 & \\
\hline & & 6 \\
\hline & & 0.70202 \\
\hline \multirow[t]{3}{*}{ Piga } & 4432416J03Rik & \\
\hline & & 2 \\
\hline & & 0.70521 \\
\hline \multirow[t]{3}{*}{ Piga } & Slc27a3 & \\
\hline & & 6 \\
\hline & & 0.78166 \\
\hline \multirow[t]{3}{*}{ Piga } & Ces1 & \\
\hline & & 2 \\
\hline & & 0.84729 \\
\hline \multirow[t]{2}{*}{ Piga } & Gins2 & \\
\hline & & 6 \\
\hline
\end{tabular}




\begin{tabular}{|c|c|c|}
\hline \multirow[t]{2}{*}{ Piga } & D11Lgp2e & $\begin{array}{c}0.78286 \\
2\end{array}$ \\
\hline & & 0.73787 \\
\hline \multirow[t]{3}{*}{ Piga } & Bcdo2 & \\
\hline & & 1 \\
\hline & & 0.72641 \\
\hline \multirow[t]{3}{*}{ Piga } & 2610042O14Rik & \\
\hline & & 7 \\
\hline & & 0.70264 \\
\hline \multirow[t]{3}{*}{ Piga } & Rgs12 & \\
\hline & & 1 \\
\hline & & 0.92026 \\
\hline \multirow[t]{3}{*}{ Piga } & Gpr152 & \\
\hline & & 1 \\
\hline & & 0.98104 \\
\hline \multirow[t]{3}{*}{ Psat1 } & Ptrh1 & \\
\hline & & 1 \\
\hline & & 0.87523 \\
\hline \multirow[t]{3}{*}{ Psat1 } & 6720458F09Rik & \\
\hline & & 6 \\
\hline & & 0.87758 \\
\hline \multirow[t]{3}{*}{ Psat1 } & C1qtnf1 & \\
\hline & & 2 \\
\hline & & 0.86768 \\
\hline \multirow[t]{3}{*}{ Psat1 } & Cox6a2 & \\
\hline & & 5 \\
\hline & & 0.92103 \\
\hline \multirow[t]{2}{*}{ Psat1 } & Kdelr3 & \\
\hline & & 8 \\
\hline
\end{tabular}




\begin{tabular}{|c|c|c|}
\hline \multirow[t]{2}{*}{ Psat1 } & Bcmo1 & $\begin{array}{c}0.87901 \\
8\end{array}$ \\
\hline & & 0.70959 \\
\hline \multirow[t]{3}{*}{ Psat1 } & Pmm1 & \\
\hline & & 6 \\
\hline & & 0.70579 \\
\hline \multirow[t]{3}{*}{ Psat1 } & Aox3 & \\
\hline & & 4 \\
\hline & & 0.84852 \\
\hline \multirow[t]{3}{*}{ Psat1 } & Smox & \\
\hline & & 3 \\
\hline & & 0.89807 \\
\hline \multirow[t]{3}{*}{ Psat1 } & 1810020C02Rik & \\
\hline & & 2 \\
\hline & & 0.77552 \\
\hline \multirow[t]{3}{*}{ Psat1 } & Ttc39a & \\
\hline & & 2 \\
\hline & & 0.93888 \\
\hline \multirow[t]{3}{*}{ Psat1 } & Mcf2I & \\
\hline & & 3 \\
\hline & & 0.72880 \\
\hline \multirow[t]{2}{*}{ Psat1 } & LOC219106 & \\
\hline & & 7 \\
\hline \multirow[t]{2}{*}{ Psat1 } & Guca2a & 0.89617 \\
\hline & & 0.87300 \\
\hline \multirow[t]{3}{*}{ Psat1 } & 4833403D03Rik & \\
\hline & & 4 \\
\hline & & 0.70728 \\
\hline \multirow[t]{2}{*}{ Psat1 } & Bcdo2 & \\
\hline & & 5 \\
\hline
\end{tabular}




\begin{tabular}{|c|c|c|}
\hline Psat1 & 5730410E15Rik & $\begin{array}{c}0.72739 \\
5\end{array}$ \\
\hline Psat1 & Zfp800 & $\begin{array}{c}0.81197 \\
9\end{array}$ \\
\hline 1110046J11Rik & Abcg8 & $\begin{array}{c}0.82897 \\
4\end{array}$ \\
\hline 1110046J11Rik & Apoa5 & $\begin{array}{c}0.86848 \\
7\end{array}$ \\
\hline 1110046J11Rik & C730029A08Rik & $\begin{array}{c}0.98055 \\
5\end{array}$ \\
\hline 1110046J11Rik & LOC380998 & $\begin{array}{c}0.76753 \\
4\end{array}$ \\
\hline 1110046J11Rik & Tmie & $\begin{array}{c}0.71138 \\
6\end{array}$ \\
\hline 1110046J11Rik & 3010026009Rik & $\begin{array}{c}0.78153 \\
5\end{array}$ \\
\hline 1110046J11Rik & Pdzk1ip1 & $\begin{array}{c}0.86360 \\
3\end{array}$ \\
\hline 1110046J11Rik & Cpsf4l & $\begin{array}{c}0.79019 \\
9\end{array}$ \\
\hline
\end{tabular}




\begin{tabular}{|c|c|c|}
\hline 1110046J11Rik & EG240549 & $\begin{array}{c}0.78611 \\
2\end{array}$ \\
\hline 1110046J11Rik & 4432416J03Rik & $\begin{array}{c}0.87088 \\
9\end{array}$ \\
\hline 1110046J11Rik & 2610528J11Rik & $\begin{array}{c}0.79191 \\
8\end{array}$ \\
\hline 1110046J11Rik & C430002P19Rik & $\begin{array}{c}0.72716 \\
3\end{array}$ \\
\hline 1110046J11Rik & D11Lgp2e & $\begin{array}{c}0.85842 \\
8\end{array}$ \\
\hline 1110046J11Rik & Ube2u & $\begin{array}{c}0.80984 \\
4\end{array}$ \\
\hline 1110046J11Rik & Cnksr1 & $\begin{array}{c}0.90312 \\
6\end{array}$ \\
\hline 1110046J11Rik & 2610042O14Rik & $\begin{array}{c}0.75552 \\
4\end{array}$ \\
\hline 1110046J11Rik & B230207L18Rik & $\begin{array}{c}0.75336 \\
6\end{array}$ \\
\hline 1700019H03Rik & BC048644 & $\begin{array}{c}0.75994 \\
4\end{array}$ \\
\hline
\end{tabular}




\begin{tabular}{|c|c|c|}
\hline 1700019H03Rik & Abcg8 & $\begin{array}{c}0.71651 \\
4\end{array}$ \\
\hline 1700019H03Rik & Proz & $\begin{array}{c}0.76435 \\
7\end{array}$ \\
\hline 1700019H03Rik & Apoa5 & $\begin{array}{c}0.77651 \\
4\end{array}$ \\
\hline 1700019H03Rik & LOC240549 & $\begin{array}{c}0.77428 \\
8\end{array}$ \\
\hline 1700019H03Rik & C730029A08Rik & $\begin{array}{c}0.72461 \\
8\end{array}$ \\
\hline 1700019H03Rik & Ung & 0.84493 \\
\hline 1700019H03Rik & Bdh1 & $\begin{array}{c}0.72113 \\
8\end{array}$ \\
\hline 1700019H03Rik & Tmem25 & $\begin{array}{c}0.74653 \\
6\end{array}$ \\
\hline 1700019H03Rik & Mist1 & $\begin{array}{c}0.93169 \\
6\end{array}$ \\
\hline 1700019H03Rik & Bhlhb8 & $\begin{array}{c}0.90577 \\
5\end{array}$ \\
\hline 1700019H03Rik & Slc25a42 & $\begin{array}{c}0.87558 \\
2\end{array}$ \\
\hline
\end{tabular}




\begin{tabular}{|c|c|c|}
\hline 1700019H03Rik & Pmm1 & 0.84013 \\
\hline & & 0.73227 \\
\hline \multirow[t]{3}{*}{ 1700019H03Rik } & Smox & \\
\hline & & 3 \\
\hline & & 0.94273 \\
\hline \multirow[t]{3}{*}{ 1700019H03Rik } & LOC433886 & \\
\hline & & 8 \\
\hline & & 0.72647 \\
\hline \multirow[t]{3}{*}{ 1700019H03Rik } & Ngef & \\
\hline & & 7 \\
\hline & & 0.86484 \\
\hline \multirow[t]{3}{*}{ 1700019H03Rik } & Slc41a3 & \\
\hline & & 7 \\
\hline & & 0.72071 \\
\hline \multirow[t]{3}{*}{ 1700019H03Rik } & Isg20 & \\
\hline & & 4 \\
\hline & & 0.71757 \\
\hline \multirow[t]{2}{*}{ 1700019H03Rik } & Map3k6 & \\
\hline & & 6 \\
\hline \multirow[t]{2}{*}{ 1700019H03Rik } & 4432416J03Rik & 0.72546 \\
\hline & & 0.76632 \\
\hline \multirow[t]{3}{*}{ 1700019H03Rik } & C430002P19Rik & \\
\hline & & 1 \\
\hline & & 0.72243 \\
\hline \multirow[t]{3}{*}{ 1700019H03Rik } & Gins2 & \\
\hline & & 2 \\
\hline & & 0.85403 \\
\hline \multirow[t]{2}{*}{ 1700019H03Rik } & D11Lgp2e & \\
\hline & & 3 \\
\hline 1700019H03Rik & Bcdo2 & 0.81841 \\
\hline
\end{tabular}




\begin{tabular}{|c|c|c|}
\hline 1700019H03Rik & Cnksr1 & $\begin{array}{c}0.80044 \\
1\end{array}$ \\
\hline 1700019H03Rik & 2610042O14Rik & $\begin{array}{c}0.79265 \\
9\end{array}$ \\
\hline 1700019H03Rik & E030049G20Rik & $\begin{array}{c}0.71886 \\
2\end{array}$ \\
\hline 1700019H03Rik & Gpr152 & $\begin{array}{c}0.77690 \\
2\end{array}$ \\
\hline Ptrh1 & 6720458F09Rik & $\begin{array}{c}0.92004 \\
1\end{array}$ \\
\hline Ptrh1 & C1qtnf1 & $\begin{array}{c}0.91704 \\
5\end{array}$ \\
\hline Ptrh1 & Cox6a2 & $\begin{array}{c}0.91236 \\
1\end{array}$ \\
\hline Ptrh1 & Kdelr3 & $\begin{array}{c}0.95790 \\
1\end{array}$ \\
\hline Ptrh1 & Bcmo1 & $\begin{array}{c}0.85723 \\
5\end{array}$ \\
\hline Ptrh1 & Pmm1 & $\begin{array}{c}0.76829 \\
9\end{array}$ \\
\hline
\end{tabular}




\begin{tabular}{|c|c|c|}
\hline \multirow[t]{2}{*}{ Ptrh1 } & Smox & $\begin{array}{c}0.86356 \\
2\end{array}$ \\
\hline & & 0.93317 \\
\hline \multirow[t]{3}{*}{ Ptrh1 } & 1810020C02Rik & \\
\hline & & 3 \\
\hline & & 0.75324 \\
\hline \multirow[t]{3}{*}{ Ptrh1 } & Ttc39a & \\
\hline & & 8 \\
\hline & & 0.95897 \\
\hline \multirow[t]{3}{*}{ Ptrh1 } & Mcf2I & \\
\hline & & 4 \\
\hline & & 0.70116 \\
\hline \multirow[t]{3}{*}{ Ptrh1 } & LOC219106 & \\
\hline & & 7 \\
\hline & & 0.82325 \\
\hline \multirow[t]{2}{*}{ Ptrh1 } & Guca2a & \\
\hline & & 4 \\
\hline \multirow[t]{2}{*}{ Ptrh1 } & 4833403D03Rik & 0.8152 \\
\hline & & 0.73707 \\
\hline \multirow[t]{3}{*}{ Ptrh1 } & Bcdo2 & \\
\hline & & 5 \\
\hline & & 0.72049 \\
\hline \multirow[t]{3}{*}{ Ptrh1 } & Srd5a2 & \\
\hline & & 2 \\
\hline & & 0.85643 \\
\hline \multirow[t]{3}{*}{ Ptrh1 } & Zfp800 & \\
\hline & & 8 \\
\hline & & 0.84989 \\
\hline \multirow[t]{2}{*}{ Agxt2I1 } & LOC240549 & \\
\hline & & 5 \\
\hline
\end{tabular}




\begin{tabular}{|c|c|c|}
\hline Agxt2I1 & Pbld & $\begin{array}{c}0.76588 \\
7\end{array}$ \\
\hline Agxt2l1 & Tmem25 & $\begin{array}{c}0.80268 \\
8\end{array}$ \\
\hline Agxt2l1 & Mrpl49 & $\begin{array}{c}0.93565 \\
7\end{array}$ \\
\hline Agxt2|1 & Hsd3b5 & $\begin{array}{c}0.86034 \\
3\end{array}$ \\
\hline Agxt2I1 & Slc25a23 & $\begin{array}{c}0.77707 \\
8\end{array}$ \\
\hline Agxt2I1 & Fn3k & $\begin{array}{c}0.97094 \\
4\end{array}$ \\
\hline Agxt2l1 & Ngef & $\begin{array}{c}0.79280 \\
9\end{array}$ \\
\hline Agxt2I1 & Zfp750 & $\begin{array}{c}0.92009 \\
9\end{array}$ \\
\hline Agxt2I1 & Bik & $\begin{array}{c}0.73925 \\
5\end{array}$ \\
\hline Agxt2I1 & Hacl1 & $\begin{array}{c}0.77940 \\
8\end{array}$ \\
\hline Agxt2l1 & Fam129a & 0.89168 \\
\hline
\end{tabular}




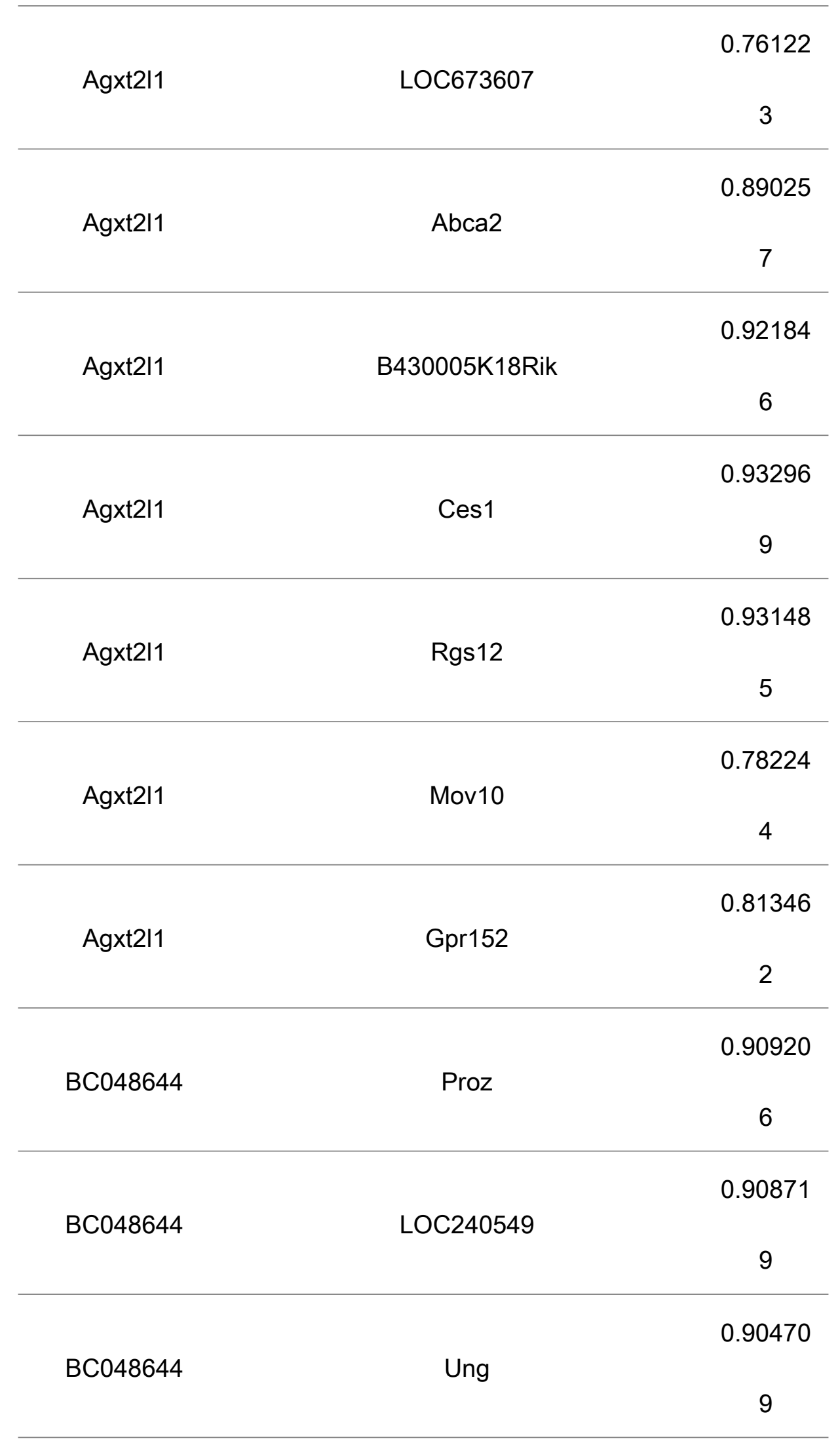




\begin{tabular}{|c|c|c|}
\hline BC048644 & Tmem25 & $\begin{array}{c}0.79018 \\
9\end{array}$ \\
\hline BC048644 & Cox6a2 & 0.71253 \\
\hline BC048644 & Ccdc120 & $\begin{array}{c}0.92646 \\
3\end{array}$ \\
\hline BC048644 & Dhcr7 & $\begin{array}{c}0.71702 \\
7\end{array}$ \\
\hline BC048644 & Mist1 & $\begin{array}{c}0.74008 \\
3\end{array}$ \\
\hline BC048644 & Gpr146 & 0.95284 \\
\hline BC048644 & Asns & $\begin{array}{c}0.72850 \\
5\end{array}$ \\
\hline BC048644 & Hpgd & $\begin{array}{c}0.72200 \\
1\end{array}$ \\
\hline BC048644 & Mrpl49 & $\begin{array}{c}0.76745 \\
6\end{array}$ \\
\hline BC048644 & Slc25a42 & $\begin{array}{c}0.96524 \\
5\end{array}$ \\
\hline BC048644 & Pmm1 & 0.82146 \\
\hline BC048644 & Hsd3b5 & $\begin{array}{c}0.92051 \\
1\end{array}$ \\
\hline
\end{tabular}




\begin{tabular}{|c|c|c|}
\hline \multirow[t]{2}{*}{ BC048644 } & Aox3 & $\begin{array}{c}0.76910 \\
9\end{array}$ \\
\hline & & 0.85667 \\
\hline \multirow[t]{3}{*}{ BC048644 } & Smox & \\
\hline & & 6 \\
\hline & & 0.81961 \\
\hline \multirow[t]{3}{*}{ BC048644 } & LOC433886 & \\
\hline & & 8 \\
\hline & & 0.75889 \\
\hline \multirow[t]{3}{*}{ BC048644 } & Fn3k & \\
\hline & & 6 \\
\hline & & 0.87814 \\
\hline \multirow[t]{3}{*}{ BC048644 } & Ngef & \\
\hline & & 5 \\
\hline & & 0.82122 \\
\hline \multirow[t]{3}{*}{ BC048644 } & Zfp750 & \\
\hline & & 7 \\
\hline & & 0.76592 \\
\hline \multirow[t]{3}{*}{ BC048644 } & 1810020C02Rik & \\
\hline & & 6 \\
\hline & & 0.86960 \\
\hline \multirow[t]{3}{*}{ BC048644 } & Slc41a3 & \\
\hline & & 7 \\
\hline & & 0.73999 \\
\hline \multirow[t]{3}{*}{ BC048644 } & Sel1h & \\
\hline & & 9 \\
\hline & & 0.77545 \\
\hline \multirow[t]{2}{*}{ BC048644 } & Fam129a & \\
\hline & & 5 \\
\hline
\end{tabular}




\begin{tabular}{|c|c|c|}
\hline \multirow[t]{2}{*}{ BC048644 } & B430005K18Rik & $\begin{array}{c}0.72801 \\
5\end{array}$ \\
\hline & & 0.81458 \\
\hline \multirow[t]{2}{*}{ BC048644 } & Gas7 & \\
\hline & & 3 \\
\hline \multirow[t]{2}{*}{ BC048644 } & C430002P19Rik & 0.73398 \\
\hline & & 0.86102 \\
\hline \multirow[t]{3}{*}{ BC048644 } & Ces1 & \\
\hline & & 1 \\
\hline & & 0.73196 \\
\hline \multirow[t]{3}{*}{ BC048644 } & 4833403D03Rik & \\
\hline & & 6 \\
\hline & & 0.73893 \\
\hline \multirow[t]{2}{*}{ BC048644 } & Bves & \\
\hline & & 6 \\
\hline \multirow[t]{2}{*}{ BC048644 } & Bcdo2 & 0.83065 \\
\hline & & 0.75016 \\
\hline \multirow[t]{3}{*}{ BC048644 } & Gpr152 & \\
\hline & & 1 \\
\hline & & 0.75819 \\
\hline \multirow[t]{3}{*}{ Abcg8 } & Proz & \\
\hline & & 5 \\
\hline & & 0.76804 \\
\hline \multirow[t]{3}{*}{ Abcg8 } & Apoa5 & \\
\hline & & 8 \\
\hline & & 0.87895 \\
\hline \multirow[t]{2}{*}{ Abcg8 } & C730029A08Rik & \\
\hline & & 8 \\
\hline
\end{tabular}




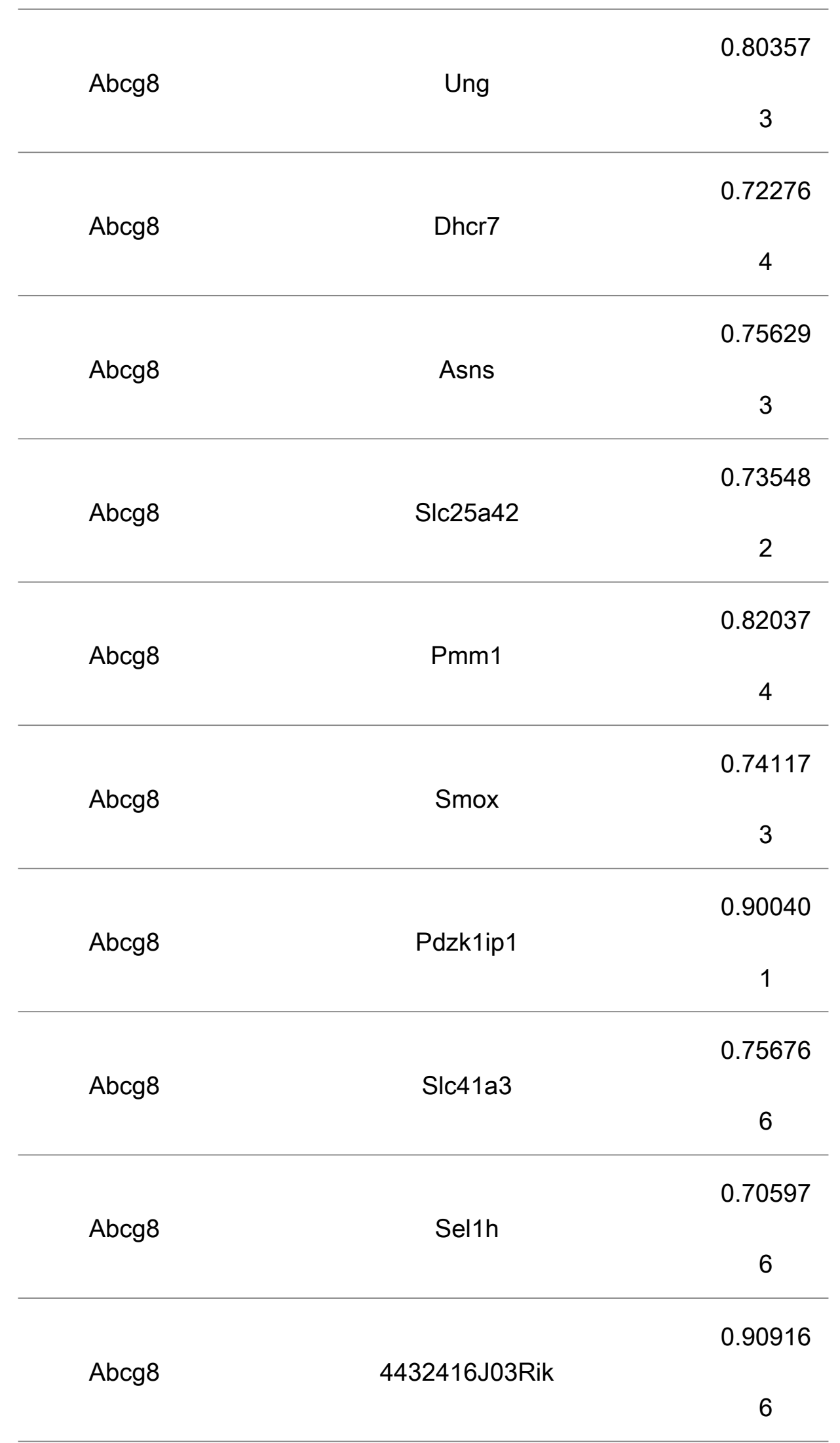




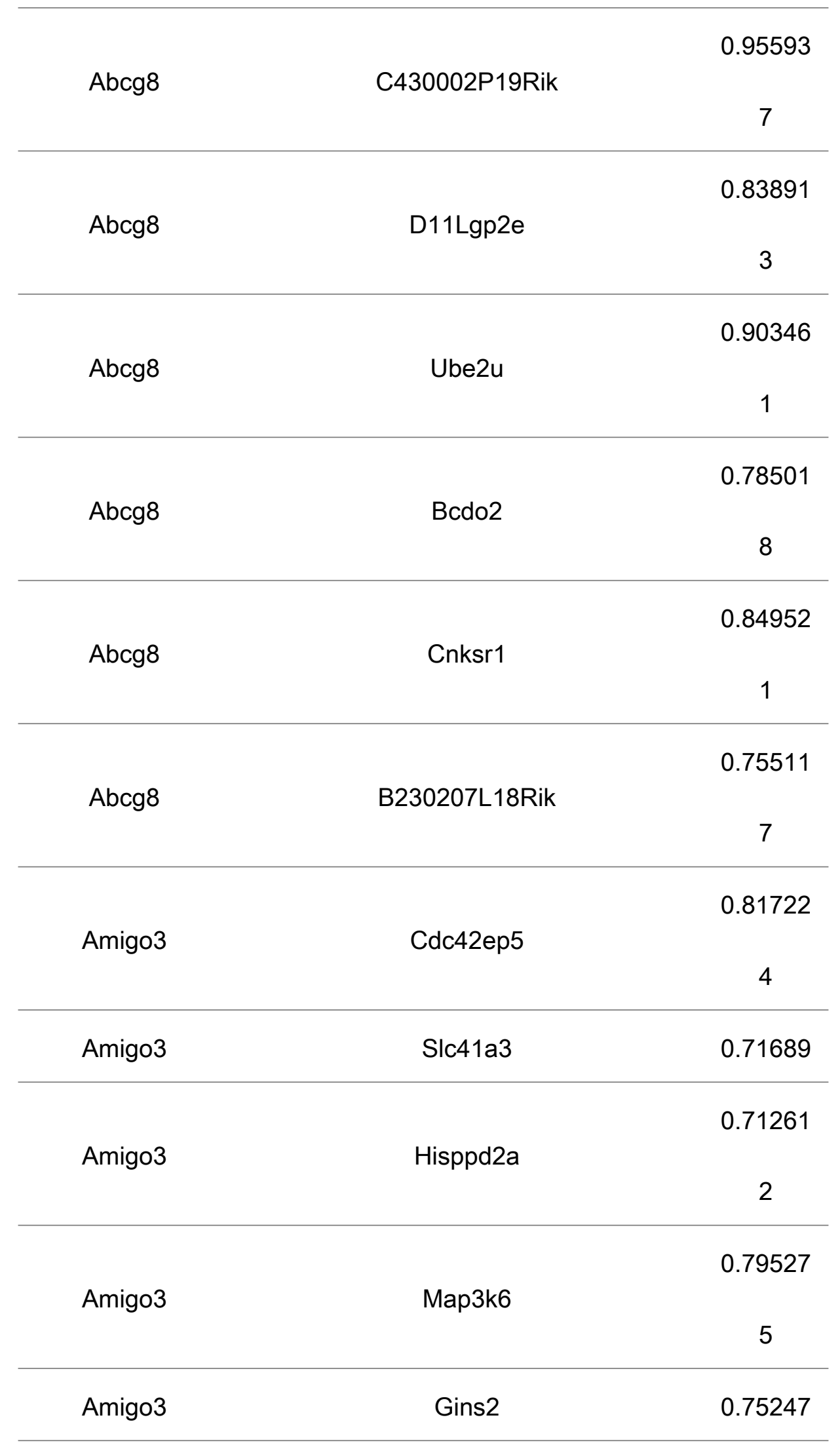




\begin{tabular}{|c|c|c|}
\hline \multirow[t]{2}{*}{ Olfml1 } & Sds & $\begin{array}{c}0.90456 \\
5\end{array}$ \\
\hline & & 0.81970 \\
\hline \multirow[t]{3}{*}{ Olfml1 } & Car14 & \\
\hline & & 3 \\
\hline & & 0.89244 \\
\hline \multirow{2}{*}{ Olfml1 } & Atp8b1 & \\
\hline & & 4 \\
\hline \multirow[t]{2}{*}{ Olfml1 } & Lrtm1 & 0.72067 \\
\hline & & 0.85648 \\
\hline \multirow[t]{3}{*}{ Olfml1 } & 9130409I23Rik & \\
\hline & & 7 \\
\hline & & 0.70481 \\
\hline \multirow[t]{3}{*}{ Olfml1 } & C430002P19Rik & \\
\hline & & 8 \\
\hline & & 0.79158 \\
\hline \multirow[t]{2}{*}{ Proz } & Apoa5 & \\
\hline & & 6 \\
\hline \multirow[t]{2}{*}{ Proz } & LOC240549 & 0.93517 \\
\hline & & 0.97828 \\
\hline \multirow[t]{3}{*}{ Proz } & Ung & \\
\hline & & 1 \\
\hline & & 0.87215 \\
\hline \multirow[t]{3}{*}{ Proz } & Bdh1 & \\
\hline & & 5 \\
\hline & & 0.91120 \\
\hline \multirow[t]{2}{*}{ Proz } & Tmem25 & \\
\hline & & 1 \\
\hline
\end{tabular}




\begin{tabular}{|c|c|c|}
\hline \multirow[t]{2}{*}{ Proz } & Ccdc120 & $\begin{array}{c}0.96637 \\
7\end{array}$ \\
\hline & & 0.92432 \\
\hline \multirow[t]{3}{*}{ Proz } & Dhcr7 & \\
\hline & & 1 \\
\hline & & 0.77300 \\
\hline \multirow[t]{3}{*}{ Proz } & Gpr146 & \\
\hline & & 8 \\
\hline & & 0.91276 \\
\hline \multirow[t]{3}{*}{ Proz } & Asns & \\
\hline & & 8 \\
\hline & & 0.87758 \\
\hline \multirow[t]{3}{*}{ Proz } & Abca8a & \\
\hline & & 5 \\
\hline & & 0.81575 \\
\hline \multirow[t]{3}{*}{ Proz } & Hpgd & \\
\hline & & 9 \\
\hline & & 0.85647 \\
\hline \multirow[t]{2}{*}{ Proz } & Mrpl49 & \\
\hline & & 3 \\
\hline \multirow[t]{2}{*}{ Proz } & Slc25a42 & 0.87101 \\
\hline & & 0.77680 \\
\hline \multirow[t]{3}{*}{ Proz } & Pmm1 & \\
\hline & & 9 \\
\hline & & 0.87905 \\
\hline \multirow[t]{3}{*}{ Proz } & Hsd3b5 & \\
\hline & & 5 \\
\hline & & 0.91596 \\
\hline \multirow[t]{2}{*}{ Proz } & Aox3 & \\
\hline & & 2 \\
\hline
\end{tabular}




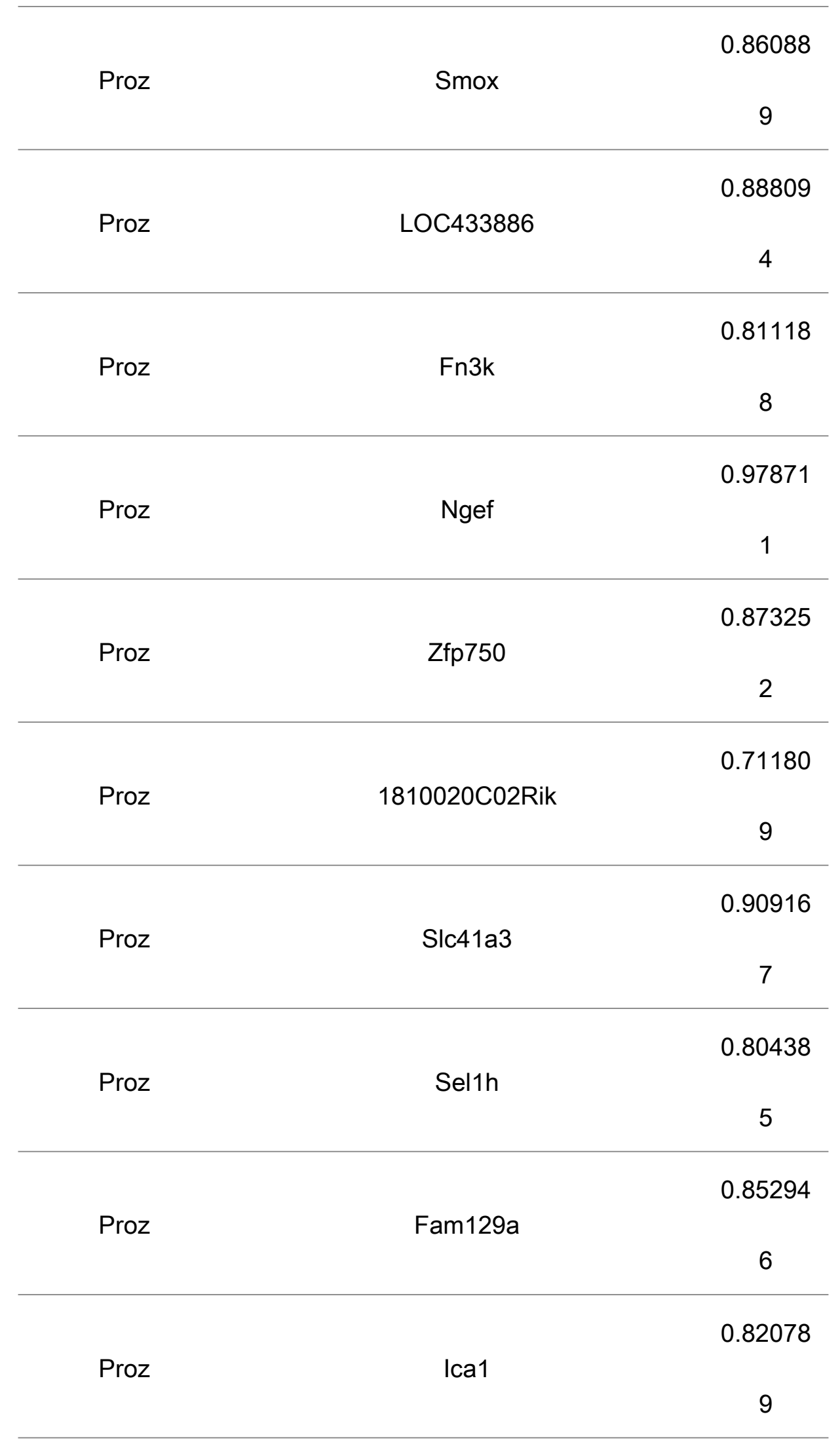




\begin{tabular}{|c|c|c|}
\hline Proz & 4432416J03Rik & $\begin{array}{c}0.79490 \\
4\end{array}$ \\
\hline Proz & Slc27a3 & $\begin{array}{c}0.79168 \\
2\end{array}$ \\
\hline Proz & C430002P19Rik & $\begin{array}{c}0.72924 \\
6\end{array}$ \\
\hline Proz & Ces1 & $\begin{array}{c}0.84363 \\
7\end{array}$ \\
\hline Proz & Gins2 & $\begin{array}{c}0.70280 \\
4\end{array}$ \\
\hline Proz & Bves & $\begin{array}{c}0.75713 \\
4\end{array}$ \\
\hline Proz & D11Lgp2e & $\begin{array}{c}0.85461 \\
8\end{array}$ \\
\hline Proz & D930009C14Rik & $\begin{array}{c}0.70639 \\
3\end{array}$ \\
\hline Proz & Bcdo2 & $\begin{array}{c}0.92730 \\
3\end{array}$ \\
\hline Proz & 5730410E15Rik & $\begin{array}{c}0.84481 \\
2\end{array}$ \\
\hline
\end{tabular}




\begin{tabular}{|c|c|c|}
\hline \multirow[t]{2}{*}{ Proz } & Gpr152 & $\begin{array}{c}0.76332 \\
6\end{array}$ \\
\hline & & 0.71219 \\
\hline \multirow[t]{3}{*}{ Apoa5 } & EG381806 & \\
\hline & & 9 \\
\hline & & 0.84906 \\
\hline \multirow[t]{3}{*}{ Apoa5 } & C730029A08Rik & \\
\hline & & 7 \\
\hline & & 0.81186 \\
\hline \multirow[t]{3}{*}{ Apoa5 } & LOC380998 & \\
\hline & & 5 \\
\hline & & 0.81417 \\
\hline \multirow[t]{3}{*}{ Apoa5 } & Ung & \\
\hline & & 1 \\
\hline & & 0.94138 \\
\hline \multirow[t]{3}{*}{ Apoa5 } & Bdh1 & \\
\hline & & 3 \\
\hline & & 0.78113 \\
\hline \multirow[t]{3}{*}{ Apoa5 } & Tmem25 & \\
\hline & & 6 \\
\hline & & 0.90513 \\
\hline \multirow[t]{3}{*}{ Apoa5 } & Dhcr7 & \\
\hline & & 4 \\
\hline & & 0.78069 \\
\hline \multirow[t]{3}{*}{ Apoa5 } & Asns & \\
\hline & & 1 \\
\hline & & 0.77153 \\
\hline \multirow[t]{2}{*}{ Apoa5 } & Abca8a & \\
\hline & & 3 \\
\hline
\end{tabular}




\begin{tabular}{|c|c|c|}
\hline \multirow[t]{2}{*}{ Apoa5 } & 3010026009Rik & $\begin{array}{c}0.93356 \\
7\end{array}$ \\
\hline & & 0.70170 \\
\hline \multirow[t]{2}{*}{ Apoa5 } & Pmm1 & \\
\hline & & 8 \\
\hline \multirow[t]{2}{*}{ Apoa5 } & Aox3 & 0.7861 \\
\hline & & 0.71081 \\
\hline \multirow[t]{3}{*}{ Apoa5 } & Smox & \\
\hline & & 1 \\
\hline & & 0.73045 \\
\hline \multirow[t]{2}{*}{ Apoa5 } & LOC100047427 & \\
\hline & & 7 \\
\hline \multirow[t]{2}{*}{ Apoa5 } & LOC433886 & 0.83707 \\
\hline & & 0.74272 \\
\hline \multirow[t]{3}{*}{ Apoa5 } & Ngef & \\
\hline & & 8 \\
\hline & & 0.77842 \\
\hline \multirow[t]{3}{*}{ Apoa5 } & Pdzk1ip1 & \\
\hline & & 3 \\
\hline & & 0.74792 \\
\hline \multirow[t]{3}{*}{ Apoa5 } & Slc41a3 & \\
\hline & & 4 \\
\hline & & 0.75806 \\
\hline \multirow[t]{3}{*}{ Apoa5 } & EG240549 & \\
\hline & & 4 \\
\hline & & 0.72853 \\
\hline \multirow[t]{2}{*}{ Apoa5 } & Ica1 & \\
\hline & & 6 \\
\hline
\end{tabular}




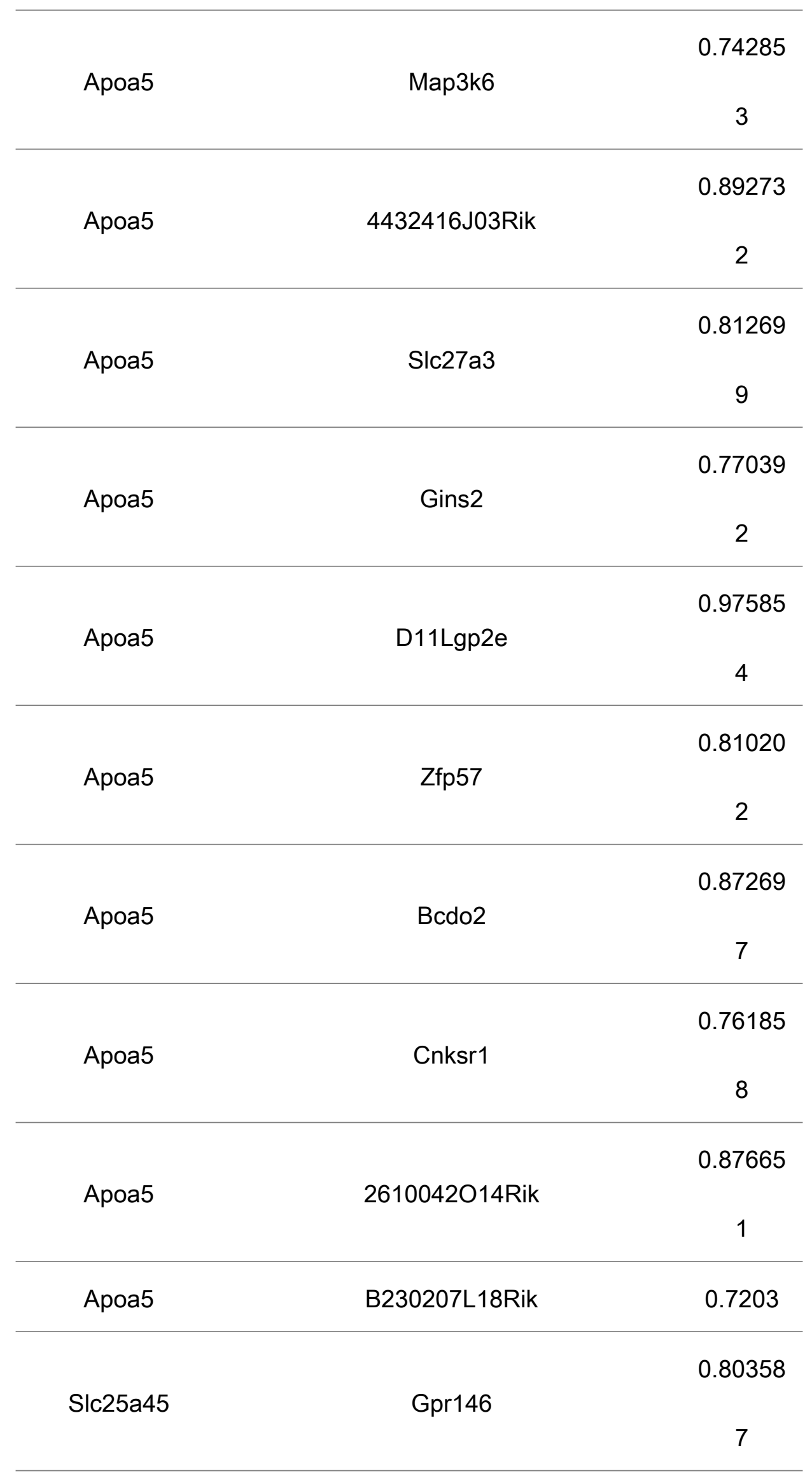




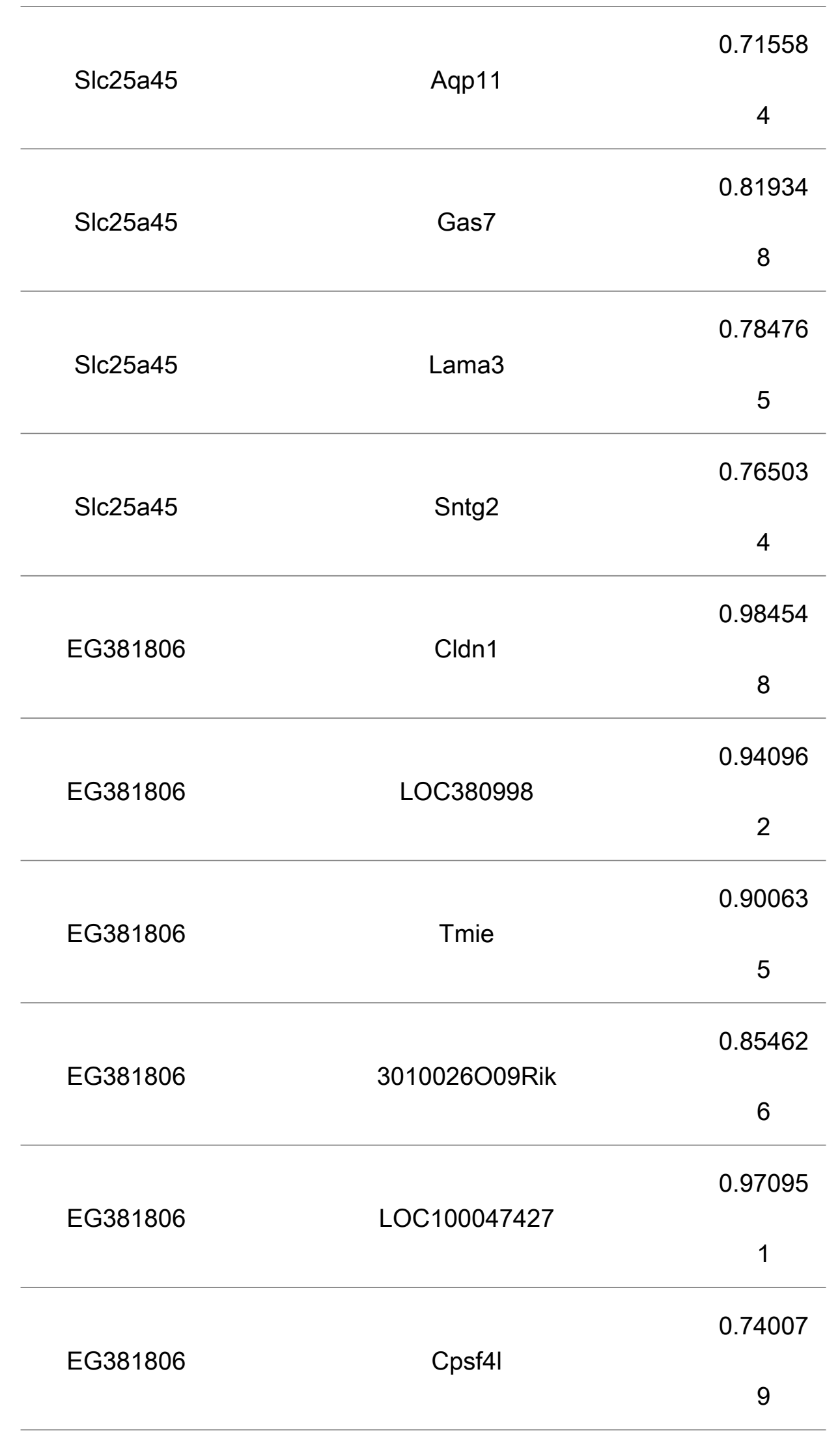




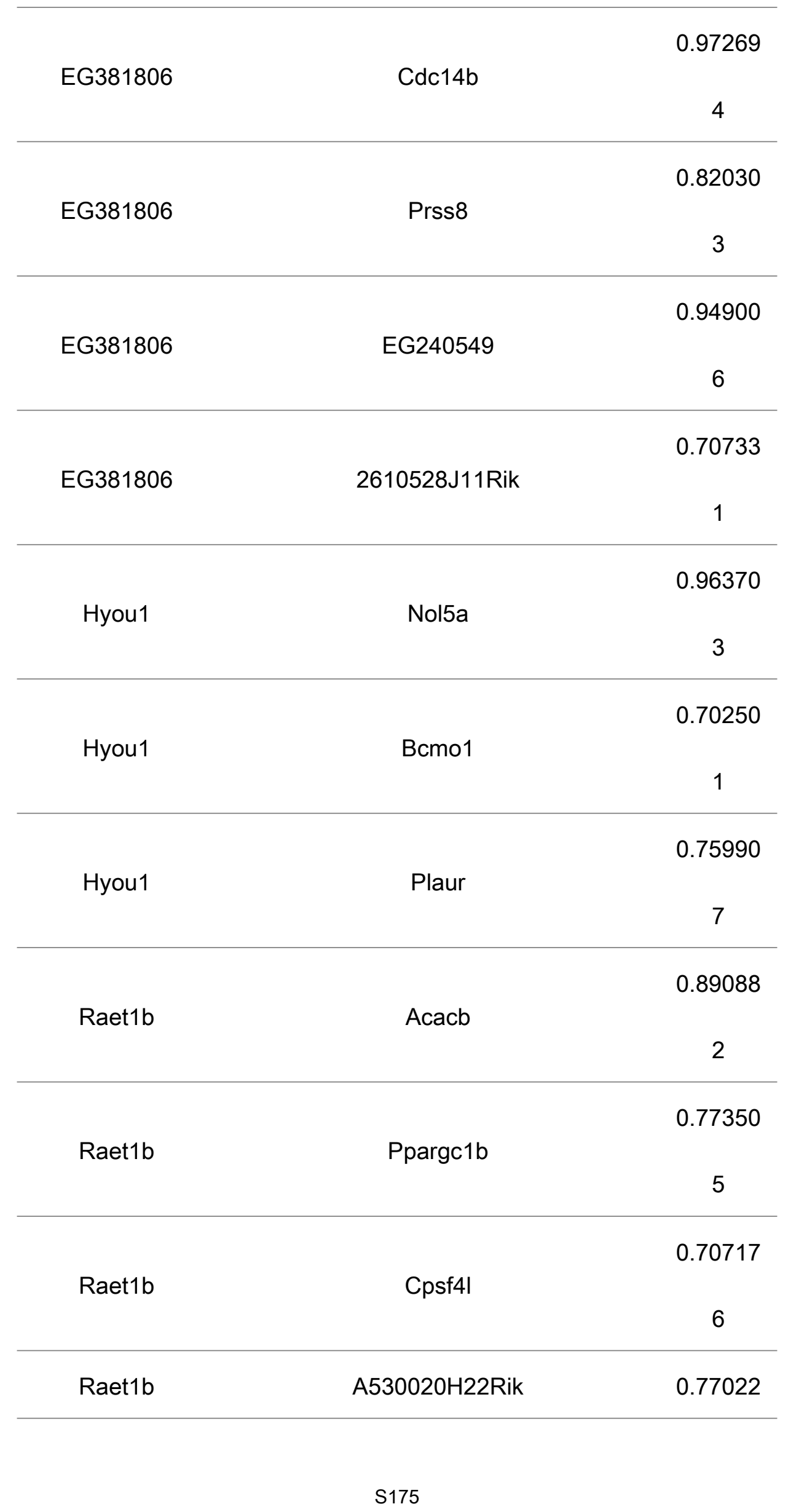




\begin{tabular}{|c|c|c|}
\hline \multirow[t]{2}{*}{ Raet1b } & 2610528J11Rik & $\begin{array}{c}0.83728 \\
2\end{array}$ \\
\hline & & 0.76599 \\
\hline \multirow[t]{3}{*}{ Raet1b } & Chrna4 & \\
\hline & & 8 \\
\hline & & 0.89992 \\
\hline \multirow[t]{3}{*}{ Cldn1 } & LOC380998 & \\
\hline & & 7 \\
\hline & & 0.90839 \\
\hline \multirow[t]{3}{*}{ Cldn1 } & Tmie & \\
\hline & & 3 \\
\hline & & 0.78321 \\
\hline \multirow[t]{3}{*}{ Cldn1 } & 3010026009Rik & \\
\hline & & 6 \\
\hline & & 0.94900 \\
\hline \multirow[t]{3}{*}{ Cldn1 } & LOC100047427 & \\
\hline & & 8 \\
\hline & & 0.77645 \\
\hline \multirow[t]{2}{*}{ Cldn1 } & Cpsf4I & \\
\hline & & 2 \\
\hline \multirow[t]{2}{*}{ Cldn1 } & Cdc14b & 0.96125 \\
\hline & & 0.73360 \\
\hline \multirow[t]{3}{*}{ Cldn1 } & Prss8 & \\
\hline & & 5 \\
\hline & & 0.94943 \\
\hline \multirow[t]{3}{*}{ Cldn1 } & EG240549 & \\
\hline & & 6 \\
\hline & & 0.74720 \\
\hline \multirow[t]{2}{*}{ Cldn1 } & 2610528J11Rik & \\
\hline & & 9 \\
\hline
\end{tabular}




\begin{tabular}{|c|c|c|}
\hline 2010305C02Rik & Lama3 & $\begin{array}{c}0.80001 \\
3\end{array}$ \\
\hline 2010305C02Rik & Sntg2 & $\begin{array}{c}0.74765 \\
1\end{array}$ \\
\hline LOC240549 & Pbld & $\begin{array}{c}0.70496 \\
8\end{array}$ \\
\hline LOC240549 & Ung & $\begin{array}{c}0.92495 \\
6\end{array}$ \\
\hline LOC240549 & Bdh1 & $\begin{array}{c}0.79226 \\
4\end{array}$ \\
\hline LOC240549 & Tmem25 & $\begin{array}{c}0.94305 \\
5\end{array}$ \\
\hline LOC240549 & Ccdc120 & $\begin{array}{c}0.89293 \\
4\end{array}$ \\
\hline LOC240549 & Dhcr7 & $\begin{array}{c}0.81031 \\
5\end{array}$ \\
\hline LOC240549 & Mist1 & $\begin{array}{c}0.73824 \\
8\end{array}$ \\
\hline LOC240549 & Gpr146 & 0.78445 \\
\hline LOC240549 & Asns & $\begin{array}{c}0.78370 \\
5\end{array}$ \\
\hline
\end{tabular}




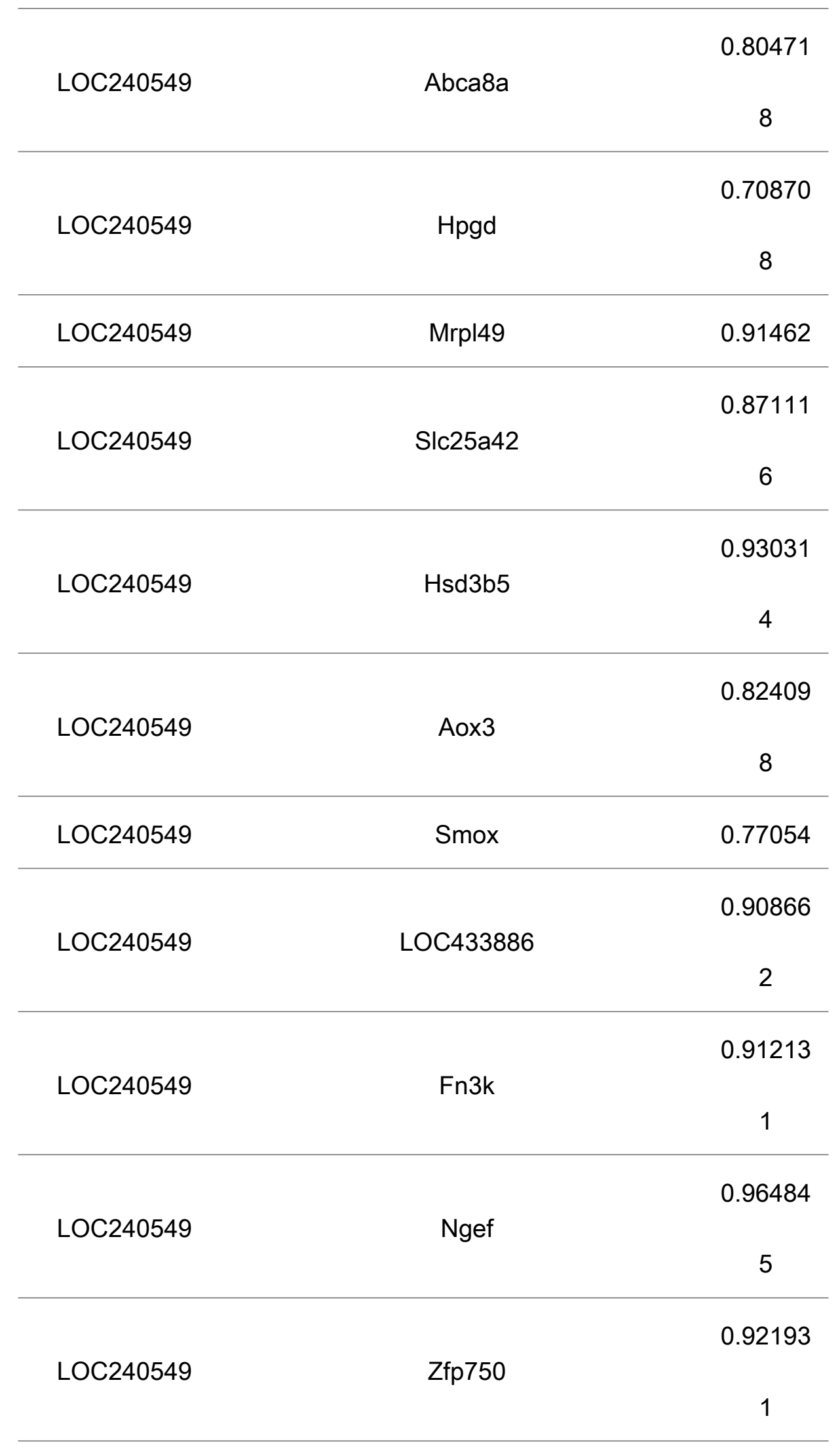




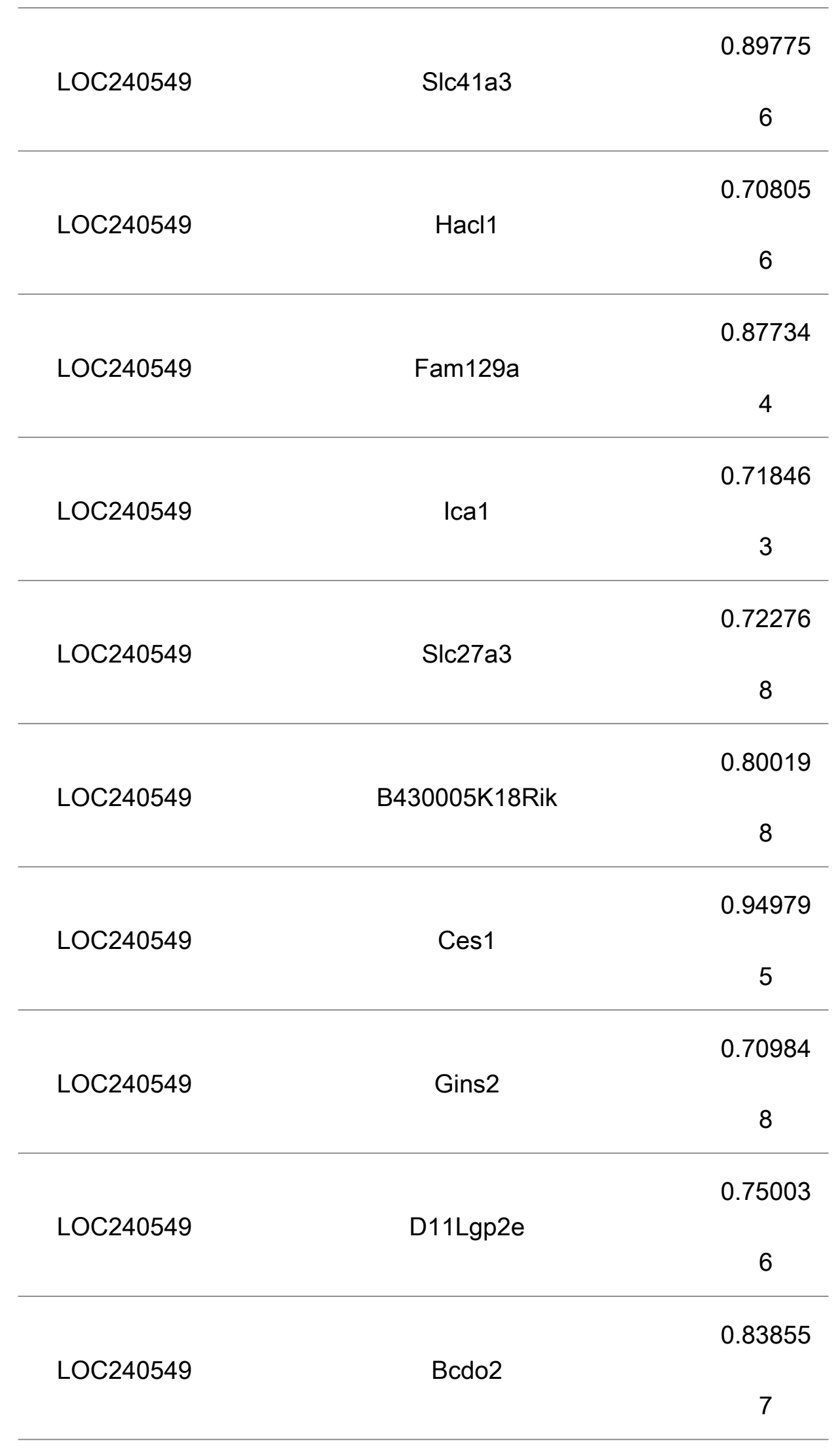




\begin{tabular}{|c|c|c|}
\hline LOC240549 & Rgs12 & $\begin{array}{c}0.80155 \\
9\end{array}$ \\
\hline LOC240549 & E030049G20Rik & $\begin{array}{c}0.77024 \\
7\end{array}$ \\
\hline LOC240549 & Gpr152 & $\begin{array}{c}0.91837 \\
3\end{array}$ \\
\hline C730029A08Rik & 3010026009Rik & $\begin{array}{c}0.73670 \\
7\end{array}$ \\
\hline C730029A08Rik & Pmm1 & $\begin{array}{c}0.75170 \\
9\end{array}$ \\
\hline C730029A08Rik & Pdzk1ip1 & $\begin{array}{c}0.83912 \\
9\end{array}$ \\
\hline C730029A08Rik & Cpsf4l & $\begin{array}{c}0.73111 \\
9\end{array}$ \\
\hline C730029A08Rik & 4432416J03Rik & $\begin{array}{c}0.85510 \\
5\end{array}$ \\
\hline C730029A08Rik & 2610528J11Rik & $\begin{array}{c}0.70160 \\
9\end{array}$ \\
\hline C730029A08Rik & C430002P19Rik & $\begin{array}{c}0.79444 \\
1\end{array}$ \\
\hline
\end{tabular}




\begin{tabular}{|c|c|c|}
\hline C730029A08Rik & D11Lgp2e & $\begin{array}{c}0.85670 \\
4\end{array}$ \\
\hline C730029A08Rik & Ube2u & $\begin{array}{c}0.89675 \\
1\end{array}$ \\
\hline C730029A08Rik & Bcdo2 & $\begin{array}{c}0.73667 \\
5\end{array}$ \\
\hline C730029A08Rik & Cnksr1 & $\begin{array}{c}0.95635 \\
1\end{array}$ \\
\hline C730029A08Rik & 2610042O14Rik & $\begin{array}{c}0.71013 \\
3\end{array}$ \\
\hline C730029A08Rik & B230207L18Rik & $\begin{array}{c}0.71496 \\
1\end{array}$ \\
\hline Acacb & Dclk3 & $\begin{array}{c}0.88723 \\
1\end{array}$ \\
\hline Acacb & Slc17a8 & $\begin{array}{c}0.76719 \\
7\end{array}$ \\
\hline Acacb & Ppargc1b & $\begin{array}{c}0.82955 \\
7\end{array}$ \\
\hline Acacb & A530020H22Rik & $\begin{array}{c}0.79606 \\
5\end{array}$ \\
\hline
\end{tabular}




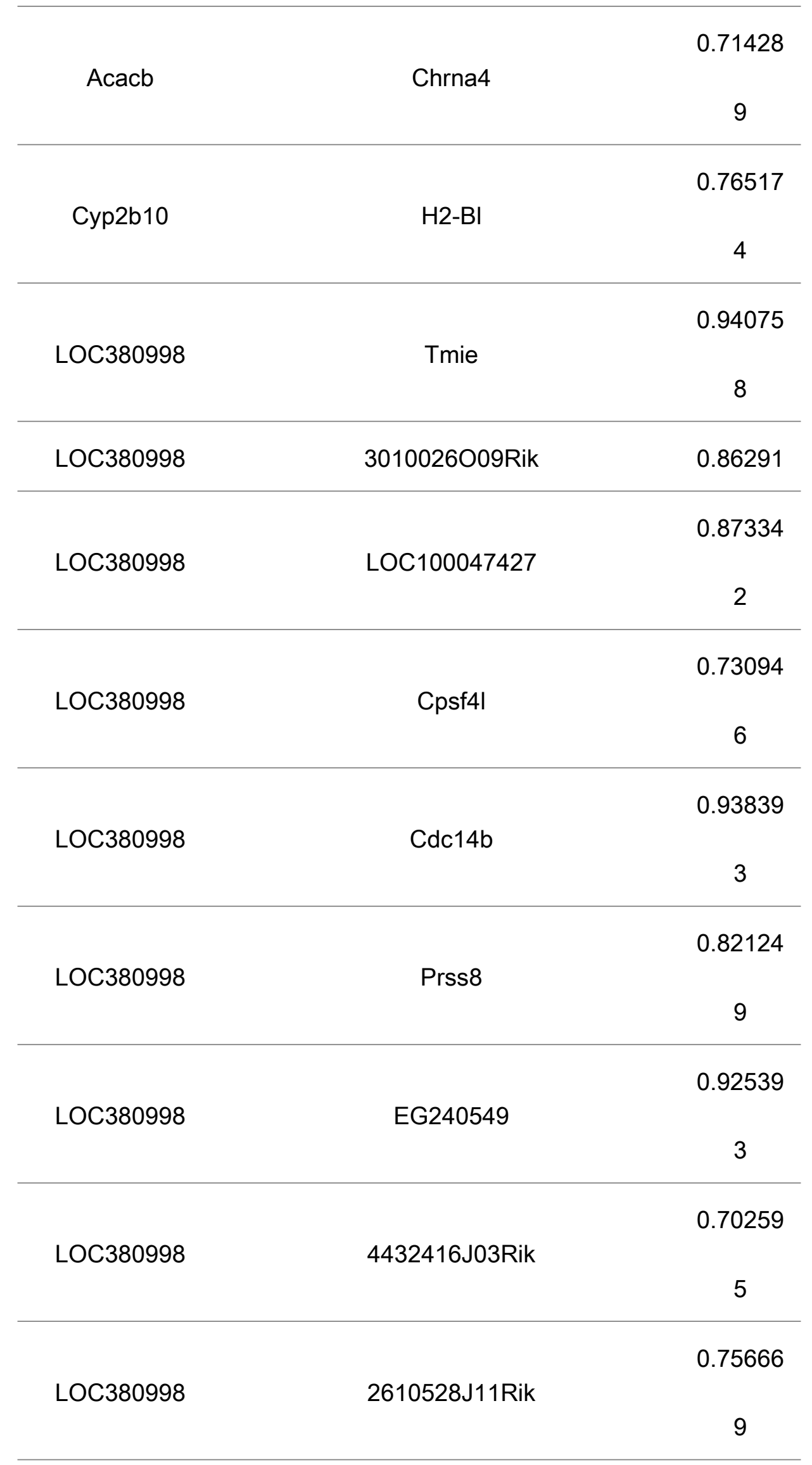




\begin{tabular}{|c|c|c|}
\hline LOC380998 & D930015E06Rik & $\begin{array}{c}0.73349 \\
9\end{array}$ \\
\hline LOC380998 & D11Lgp2e & 0.72752 \\
\hline LOC380998 & 2610042O14Rik & $\begin{array}{c}0.78431 \\
7\end{array}$ \\
\hline LOC380998 & 1810046K07Rik & $\begin{array}{c}0.70040 \\
4\end{array}$ \\
\hline Pbld & Keg1 & $\begin{array}{c}0.84462 \\
7\end{array}$ \\
\hline Pbld & Bdh1 & $\begin{array}{c}0.75905 \\
5\end{array}$ \\
\hline Pbld & Tmem25 & $\begin{array}{c}0.84753 \\
1\end{array}$ \\
\hline Pbld & Dhcr7 & $\begin{array}{c}0.73444 \\
9\end{array}$ \\
\hline Pbld & Abca8a & $\begin{array}{c}0.87017 \\
6\end{array}$ \\
\hline Pbld & Mrpl49 & $\begin{array}{c}0.85389 \\
9\end{array}$ \\
\hline Pbld & Aox3 & $\begin{array}{c}0.78873 \\
1\end{array}$ \\
\hline
\end{tabular}




\begin{tabular}{|c|c|c|}
\hline Pbld & Fn3k & $\begin{array}{c}0.83591 \\
3\end{array}$ \\
\hline Pbld & Ngef & 0.7056 \\
\hline Pbld & Zfp750 & $\begin{array}{c}0.79575 \\
5\end{array}$ \\
\hline Pbld & Tjp3 & $\begin{array}{c}0.81206 \\
4\end{array}$ \\
\hline Pbld & Hacl1 & $\begin{array}{c}0.86336 \\
4\end{array}$ \\
\hline Pbld & Fam129a & $\begin{array}{c}0.82353 \\
8\end{array}$ \\
\hline Pbld & Cabc1 & $\begin{array}{c}0.81646 \\
8\end{array}$ \\
\hline Pbld & Slc44a3 & $\begin{array}{c}0.84427 \\
8\end{array}$ \\
\hline Pbld & Ces1 & $\begin{array}{c}0.74010 \\
2\end{array}$ \\
\hline Pbld & Grb14 & $\begin{array}{c}0.79974 \\
3\end{array}$ \\
\hline Pbld & Zfp57 & $\begin{array}{c}0.79610 \\
2\end{array}$ \\
\hline
\end{tabular}




\begin{tabular}{|c|c|c|}
\hline Pbld & Acpp & $\begin{array}{c}0.75474 \\
9\end{array}$ \\
\hline Pbld & Nphp3 & $\begin{array}{c}0.71928 \\
2\end{array}$ \\
\hline Pbld & LOC381738 & $\begin{array}{c}0.76502 \\
9\end{array}$ \\
\hline Pbld & E030049G20Rik & $\begin{array}{c}0.77166 \\
7\end{array}$ \\
\hline 6720458F09Rik & C1qtnf1 & $\begin{array}{c}0.76277 \\
6\end{array}$ \\
\hline 6720458F09Rik & Cox6a2 & 0.8425 \\
\hline 6720458F09Rik & Kdelr3 & $\begin{array}{c}0.90213 \\
2\end{array}$ \\
\hline 6720458F09Rik & Bcmo1 & $\begin{array}{c}0.81707 \\
2\end{array}$ \\
\hline 6720458F09Rik & Smox & $\begin{array}{c}0.72756 \\
7\end{array}$ \\
\hline 6720458F09Rik & 1810020C02Rik & $\begin{array}{c}0.79243 \\
5\end{array}$ \\
\hline 6720458F09Rik & Ttc39a & 0.84139 \\
\hline
\end{tabular}




\begin{tabular}{|c|c|c|}
\hline 6720458F09Rik & Mcf2I & $\begin{array}{c}0.93833 \\
9\end{array}$ \\
\hline 6720458F09Rik & Guca2a & $\begin{array}{c}0.72664 \\
6\end{array}$ \\
\hline 6720458F09Rik & Srd5a2 & $\begin{array}{c}0.72168 \\
6\end{array}$ \\
\hline 6720458F09Rik & Zfp800 & $\begin{array}{c}0.86028 \\
7\end{array}$ \\
\hline Ung & Bdh1 & $\begin{array}{c}0.84829 \\
5\end{array}$ \\
\hline Ung & Tmem25 & $\begin{array}{c}0.87697 \\
8\end{array}$ \\
\hline Ung & Ccdc120 & $\begin{array}{c}0.90906 \\
2\end{array}$ \\
\hline Ung & Dhcr7 & $\begin{array}{c}0.88526 \\
5\end{array}$ \\
\hline Ung & Mist1 & $\begin{array}{c}0.72047 \\
5\end{array}$ \\
\hline Ung & Gpr146 & 0.77477 \\
\hline Ung & Asns & $\begin{array}{c}0.90296 \\
4\end{array}$ \\
\hline
\end{tabular}




\begin{tabular}{|c|c|c|}
\hline Ung & Abca8a & $\begin{array}{c}0.79578 \\
2\end{array}$ \\
\hline Ung & Hpgd & $\begin{array}{c}0.80729 \\
5\end{array}$ \\
\hline Ung & Mrpl49 & $\begin{array}{c}0.78312 \\
6\end{array}$ \\
\hline Ung & Slc25a42 & $\begin{array}{c}0.90213 \\
6\end{array}$ \\
\hline Ung & Pmm1 & $\begin{array}{c}0.79816 \\
4\end{array}$ \\
\hline Ung & Hsd3b5 & $\begin{array}{c}0.82656 \\
7\end{array}$ \\
\hline Ung & Aox3 & $\begin{array}{c}0.83769 \\
3\end{array}$ \\
\hline Ung & Smox & $\begin{array}{c}0.82016 \\
4\end{array}$ \\
\hline Ung & LOC433886 & $\begin{array}{c}0.93804 \\
4\end{array}$ \\
\hline Ung & Fn3k & $\begin{array}{c}0.74439 \\
9\end{array}$ \\
\hline Ung & Ngef & 0.95835 \\
\hline
\end{tabular}




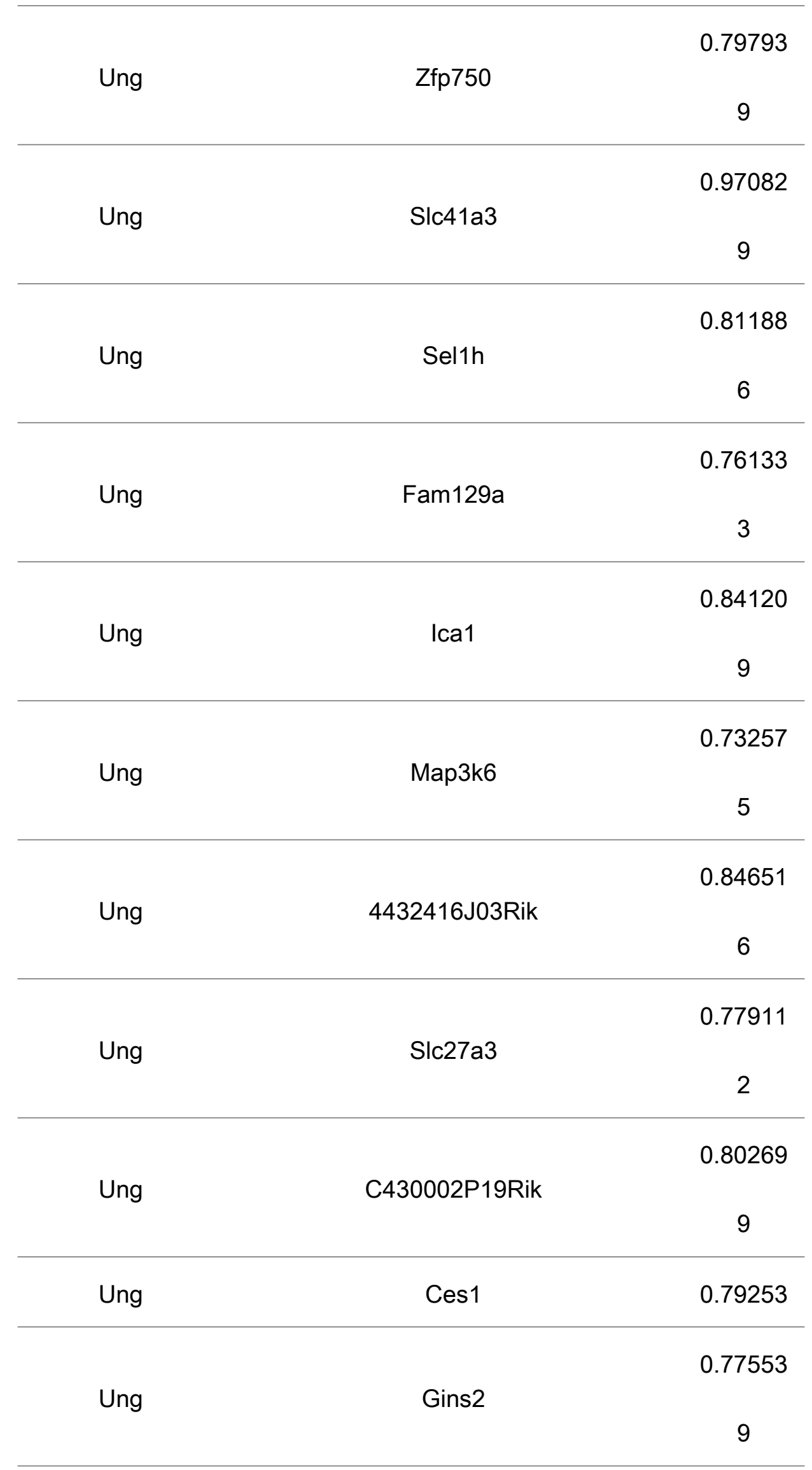




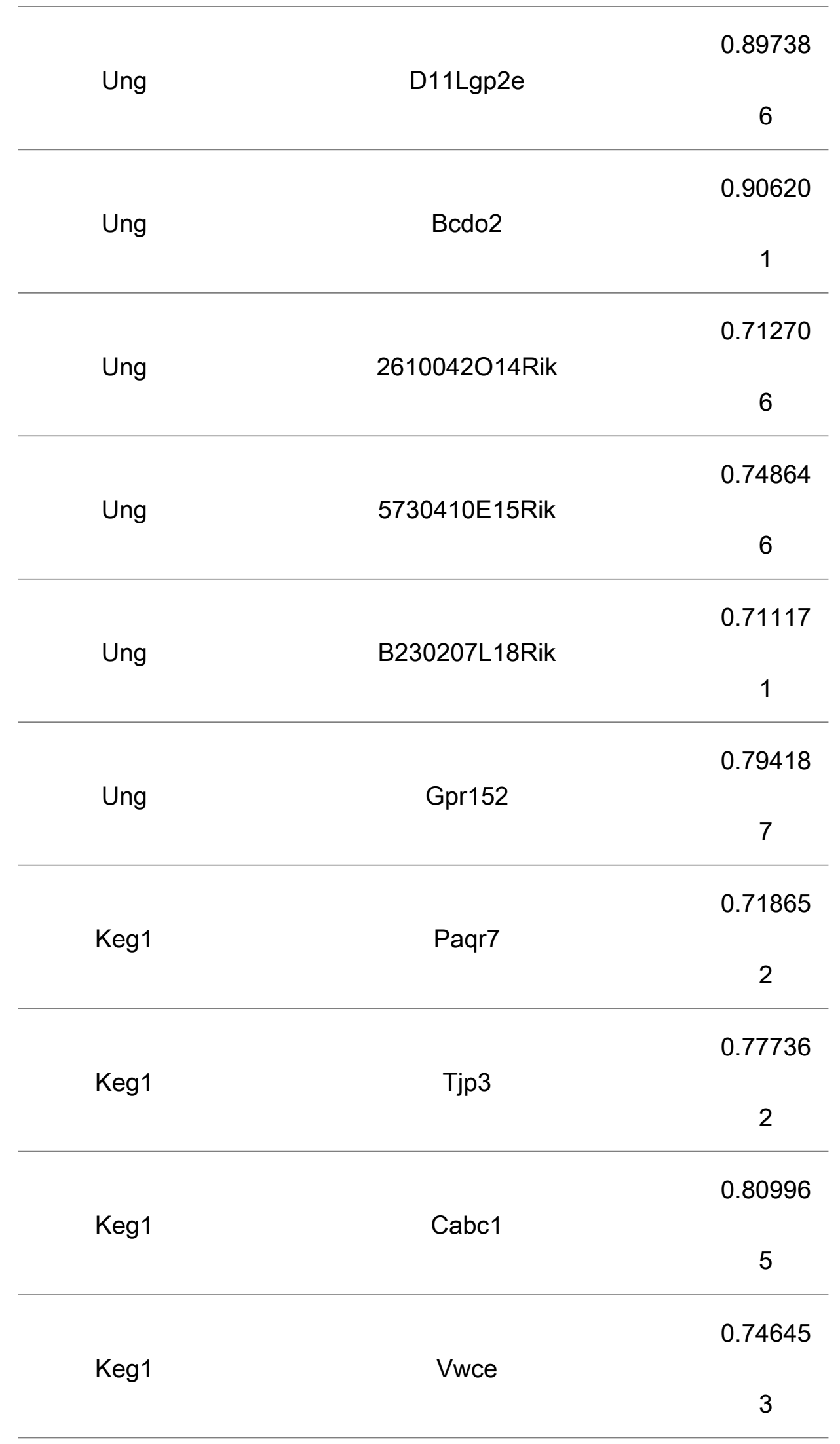




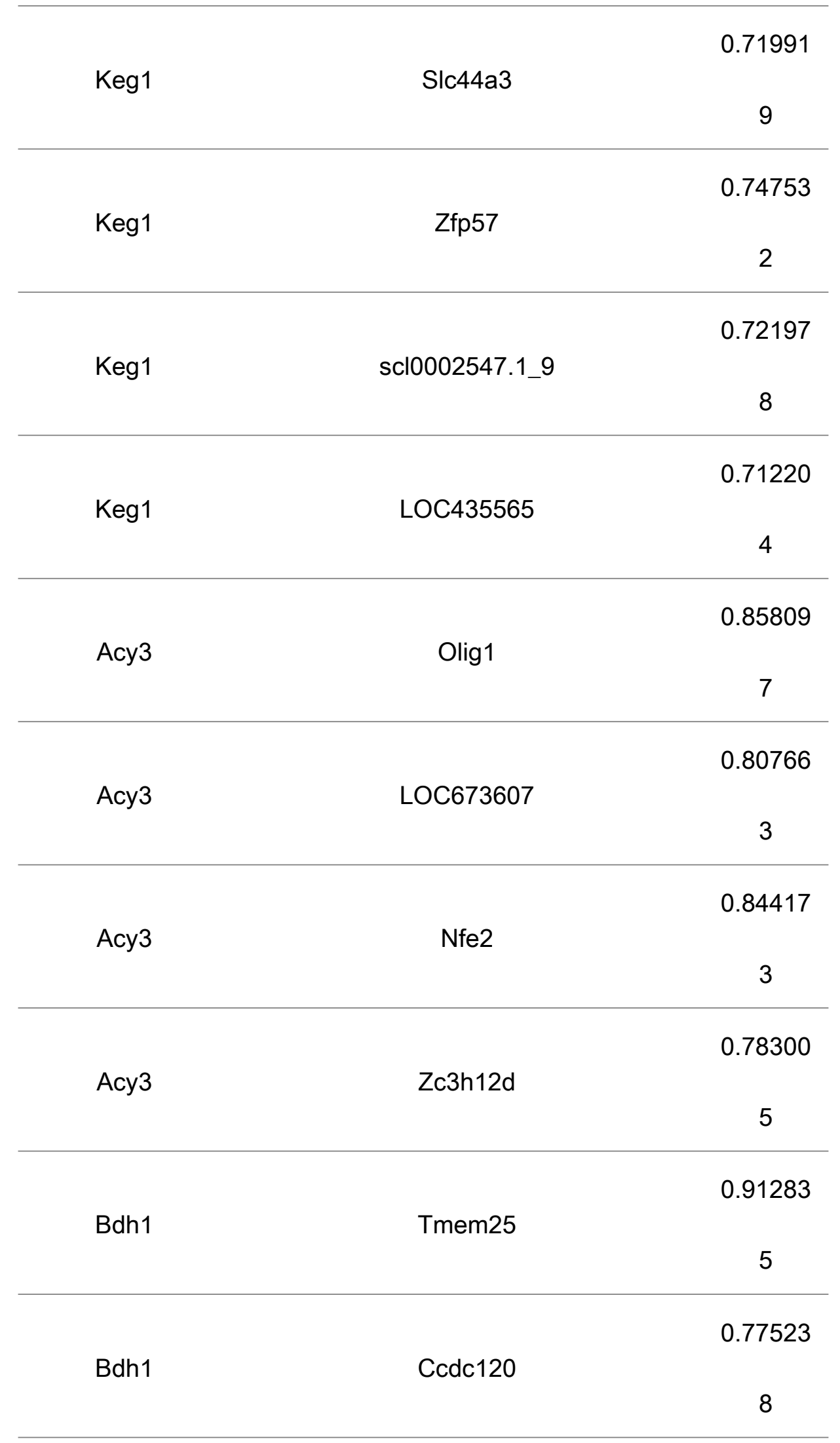




\begin{tabular}{|c|c|c|}
\hline \multirow[t]{2}{*}{ Bdh1 } & Dhcr7 & $\begin{array}{c}0.97725 \\
9\end{array}$ \\
\hline & & 0.82958 \\
\hline \multirow[t]{3}{*}{ Bdh1 } & Asns & \\
\hline & & 6 \\
\hline & & 0.92538 \\
\hline \multirow[t]{3}{*}{ Bdh1 } & Abca8a & \\
\hline & & 9 \\
\hline & & 0.75243 \\
\hline \multirow[t]{3}{*}{ Bdh1 } & Mrpl49 & \\
\hline & & 5 \\
\hline & & 0.90679 \\
\hline \multirow[t]{2}{*}{ Bdh1 } & 3010026009Rik & \\
\hline & & 7 \\
\hline \multirow[t]{2}{*}{ Bdh1 } & Paqr7 & 0.76505 \\
\hline & & 0.91030 \\
\hline \multirow[t]{3}{*}{ Bdh1 } & Aox3 & \\
\hline & & 3 \\
\hline & & 0.75915 \\
\hline \multirow[t]{3}{*}{ Bdh1 } & Smox & \\
\hline & & 3 \\
\hline & & 0.85064 \\
\hline \multirow[t]{3}{*}{ Bdh1 } & LOC433886 & \\
\hline & & 8 \\
\hline & & 0.85120 \\
\hline \multirow[t]{3}{*}{ Bdh1 } & Ngef & \\
\hline & & 6 \\
\hline & & 0.72026 \\
\hline \multirow[t]{2}{*}{ Bdh1 } & Zfp750 & \\
\hline & & 4 \\
\hline
\end{tabular}




\begin{tabular}{|c|c|c|}
\hline \multirow[t]{2}{*}{ Bdh1 } & Slc41a3 & $\begin{array}{c}0.75720 \\
5\end{array}$ \\
\hline & & 0.71392 \\
\hline \multirow[t]{3}{*}{ Bdh1 } & Prss8 & \\
\hline & & 3 \\
\hline & & 0.72122 \\
\hline \multirow[t]{3}{*}{ Bdh1 } & Fam129a & \\
\hline & & 3 \\
\hline & & 0.75537 \\
\hline \multirow[t]{3}{*}{ Bdh1 } & Ica1 & \\
\hline & & 6 \\
\hline & & 0.79875 \\
\hline \multirow[t]{3}{*}{ Bdh1 } & 4432416J03Rik & \\
\hline & & 8 \\
\hline & & 0.88876 \\
\hline \multirow[t]{3}{*}{ Bdh1 } & Slc27a3 & \\
\hline & & 2 \\
\hline & & 0.75393 \\
\hline \multirow[t]{2}{*}{ Bdh1 } & Gins2 & \\
\hline & & 5 \\
\hline \multirow[t]{2}{*}{ Bdh1 } & D11Lgp2e & 0.91515 \\
\hline & & 0.89146 \\
\hline \multirow[t]{3}{*}{ Bdh1 } & Zfp57 & \\
\hline & & 3 \\
\hline & & 0.90985 \\
\hline \multirow[t]{3}{*}{ Bdh1 } & Bcdo2 & \\
\hline & & 8 \\
\hline & & 0.78422 \\
\hline \multirow[t]{2}{*}{ Bdh1 } & 2610042O14Rik & \\
\hline & & 3 \\
\hline
\end{tabular}




\begin{tabular}{|c|c|c|}
\hline \multirow[t]{2}{*}{ Bdh1 } & 5730410E15Rik & $\begin{array}{c}0.80697 \\
3\end{array}$ \\
\hline & & 0.77832 \\
\hline \multirow[t]{3}{*}{ Bdh1 } & E030049G20Rik & \\
\hline & & 5 \\
\hline & & 0.93136 \\
\hline \multirow[t]{3}{*}{ C1qtnf1 } & Cox6a2 & \\
\hline & & 7 \\
\hline & & 0.91628 \\
\hline \multirow[t]{3}{*}{ C1qtnf1 } & Kdelr3 & \\
\hline & & 1 \\
\hline & & 0.72594 \\
\hline \multirow[t]{3}{*}{ C1qtnf1 } & Slc25a42 & \\
\hline & & 7 \\
\hline & & 0.85110 \\
\hline \multirow[t]{3}{*}{ C1qtnf1 } & Pmm1 & \\
\hline & & 6 \\
\hline & & 0.86092 \\
\hline \multirow[t]{3}{*}{ C1qtnf1 } & Smox & \\
\hline & & 2 \\
\hline & & 0.96604 \\
\hline \multirow[t]{3}{*}{ C1qtnf1 } & 1810020C02Rik & \\
\hline & & 7 \\
\hline & & 0.87499 \\
\hline \multirow[t]{2}{*}{ C1qtnf1 } & Mcf2I & \\
\hline & & 7 \\
\hline \multirow[t]{2}{*}{ C1qtnf1 } & LOC219106 & 0.79221 \\
\hline & & 0.84038 \\
\hline \multirow[t]{2}{*}{ C1qtnf1 } & 4833403D03Rik & \\
\hline & & 2 \\
\hline
\end{tabular}




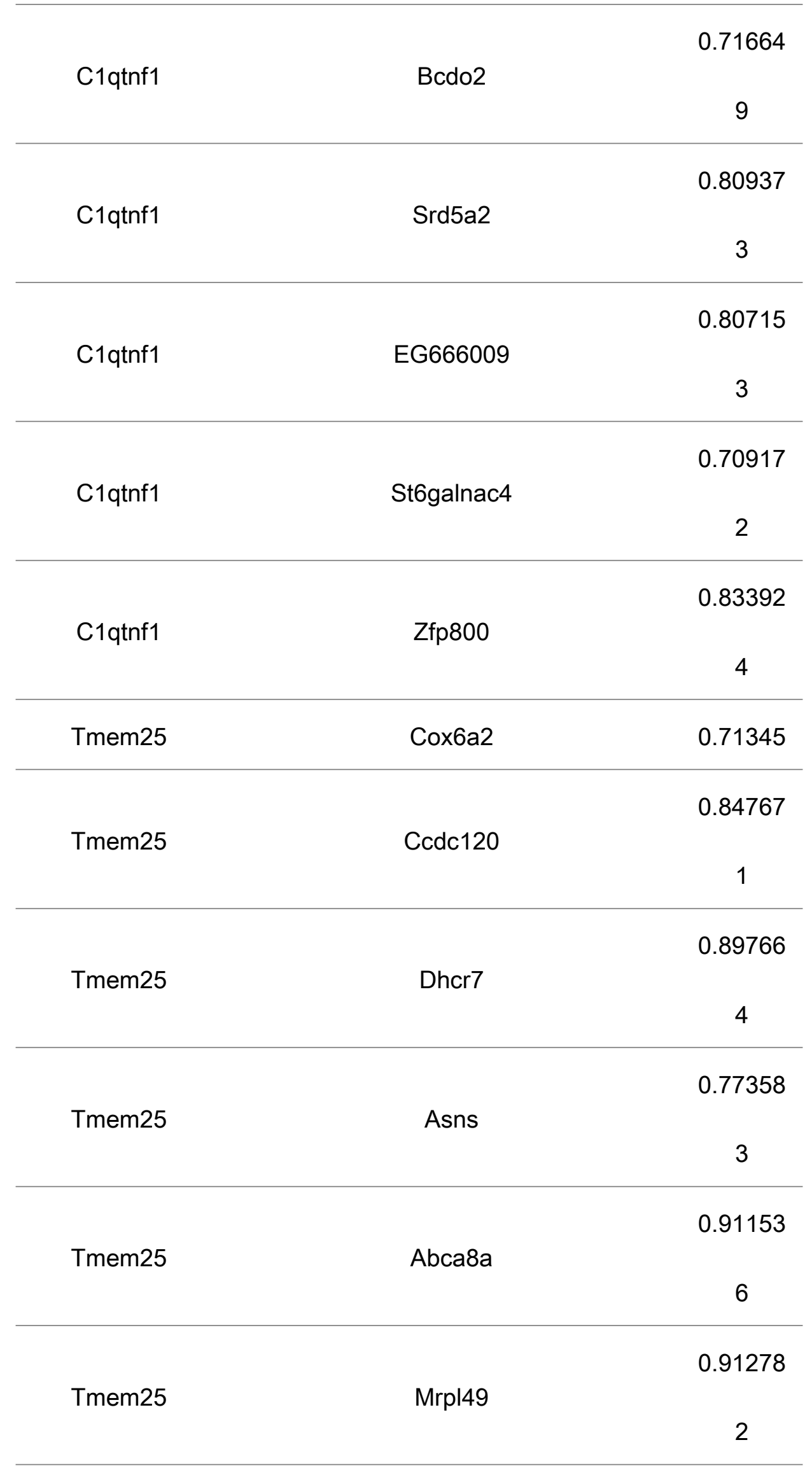




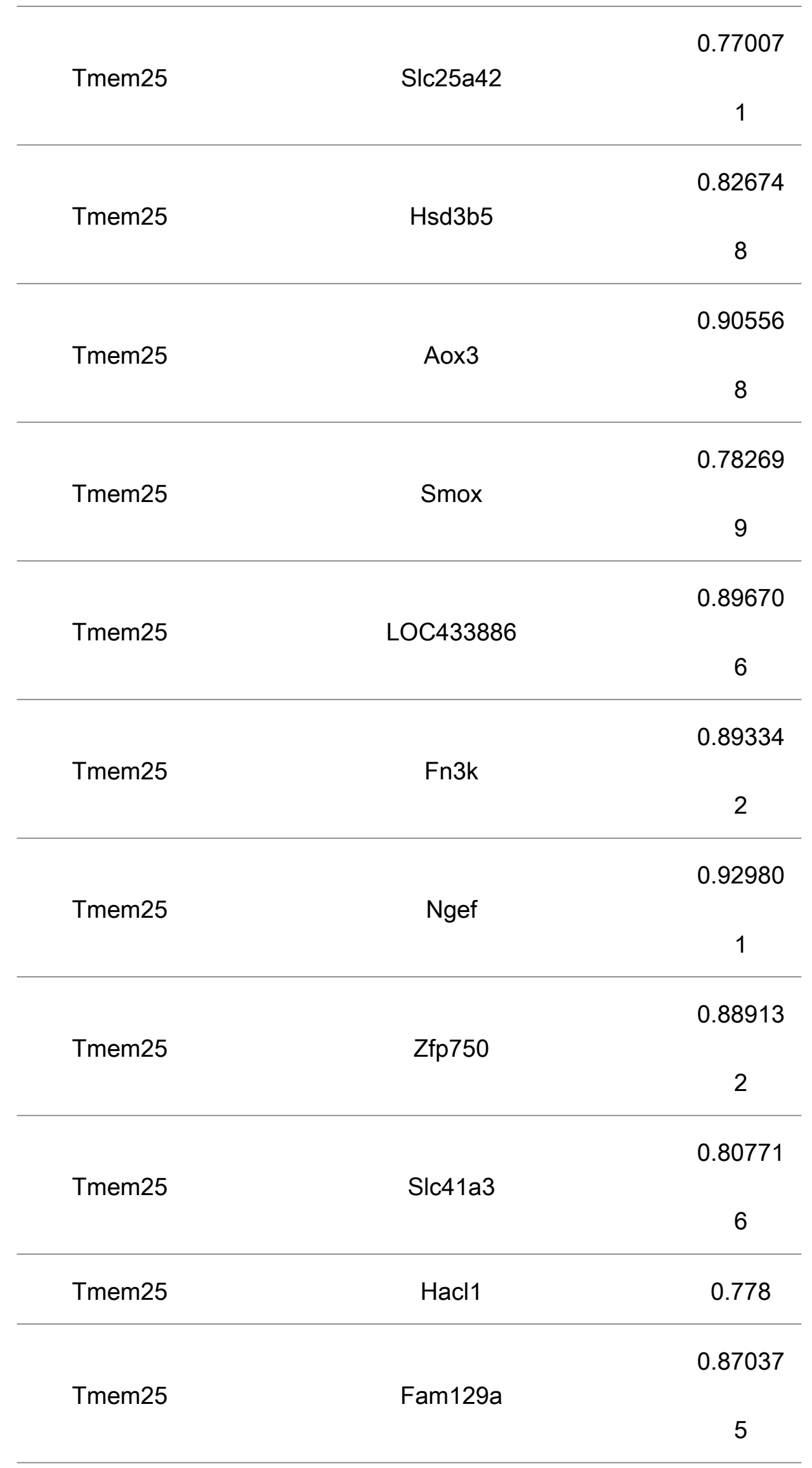




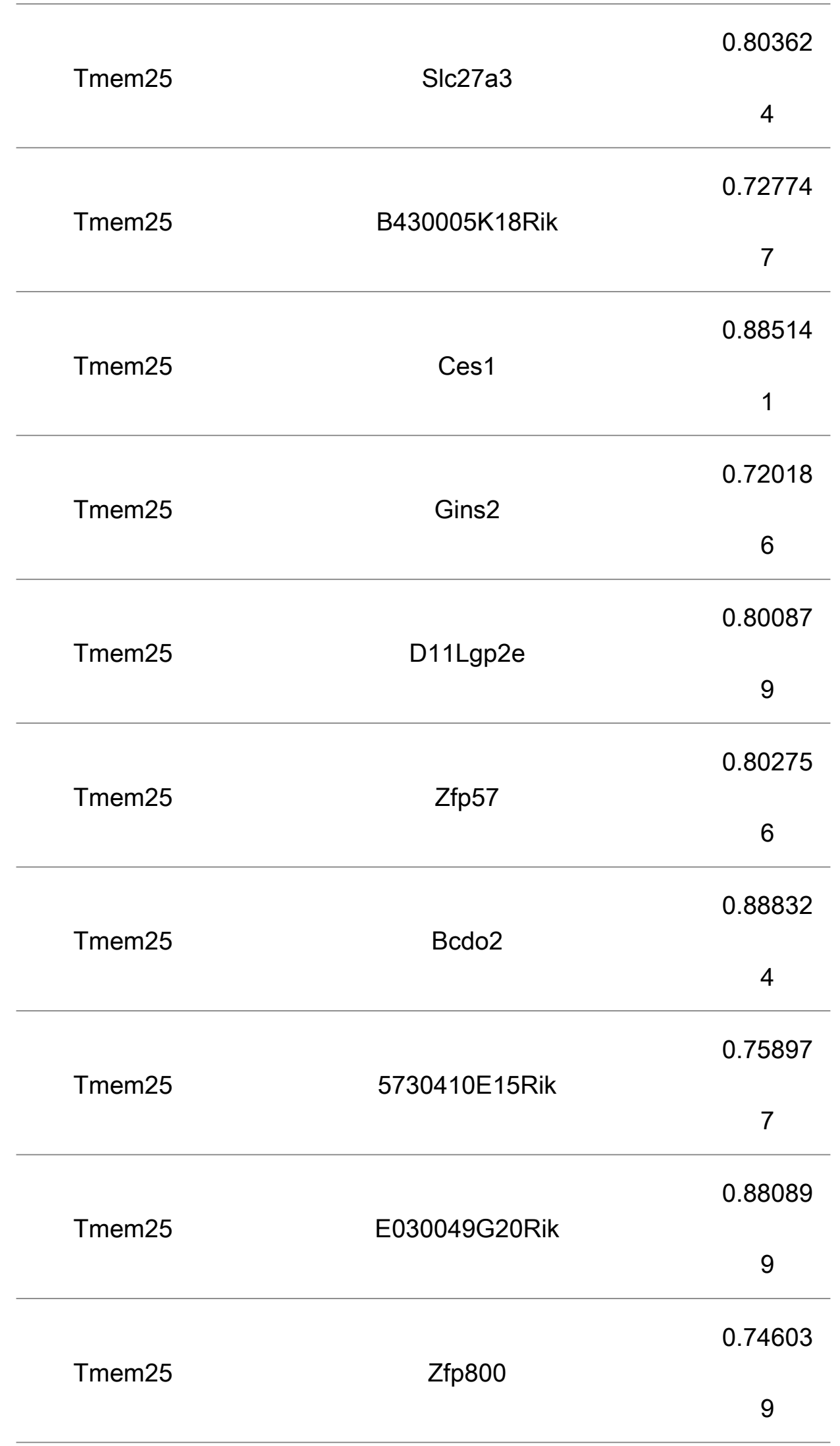




\begin{tabular}{|c|c|c|}
\hline \multirow[t]{2}{*}{ Tmem25 } & Gpr152 & $\begin{array}{c}0.86455 \\
7\end{array}$ \\
\hline & & 0.72794 \\
\hline \multirow[t]{3}{*}{ Cox6a2 } & Mist1 & \\
\hline & & 9 \\
\hline & & 0.87413 \\
\hline \multirow[t]{3}{*}{ Cox6a2 } & Kdelr3 & \\
\hline & & 4 \\
\hline & & 0.76192 \\
\hline \multirow[t]{3}{*}{ Cox6a2 } & Slc25a42 & \\
\hline & & 9 \\
\hline & & 0.85909 \\
\hline \multirow[t]{3}{*}{ Cox6a2 } & Pmm1 & \\
\hline & & 2 \\
\hline & & 0.76449 \\
\hline \multirow[t]{3}{*}{ Cox6a2 } & Aox3 & \\
\hline & & 6 \\
\hline & & 0.91783 \\
\hline \multirow[t]{3}{*}{ Cox6a2 } & Smox & \\
\hline & & 1 \\
\hline & & 0.96316 \\
\hline \multirow[t]{3}{*}{ Cox6a2 } & 1810020C02Rik & \\
\hline & & 6 \\
\hline & & 0.95506 \\
\hline \multirow[t]{3}{*}{ Cox6a2 } & Mcf2I & \\
\hline & & 8 \\
\hline & & 0.79299 \\
\hline \multirow[t]{2}{*}{ Cox6a2 } & 4833403D03Rik & \\
\hline & & 2 \\
\hline
\end{tabular}




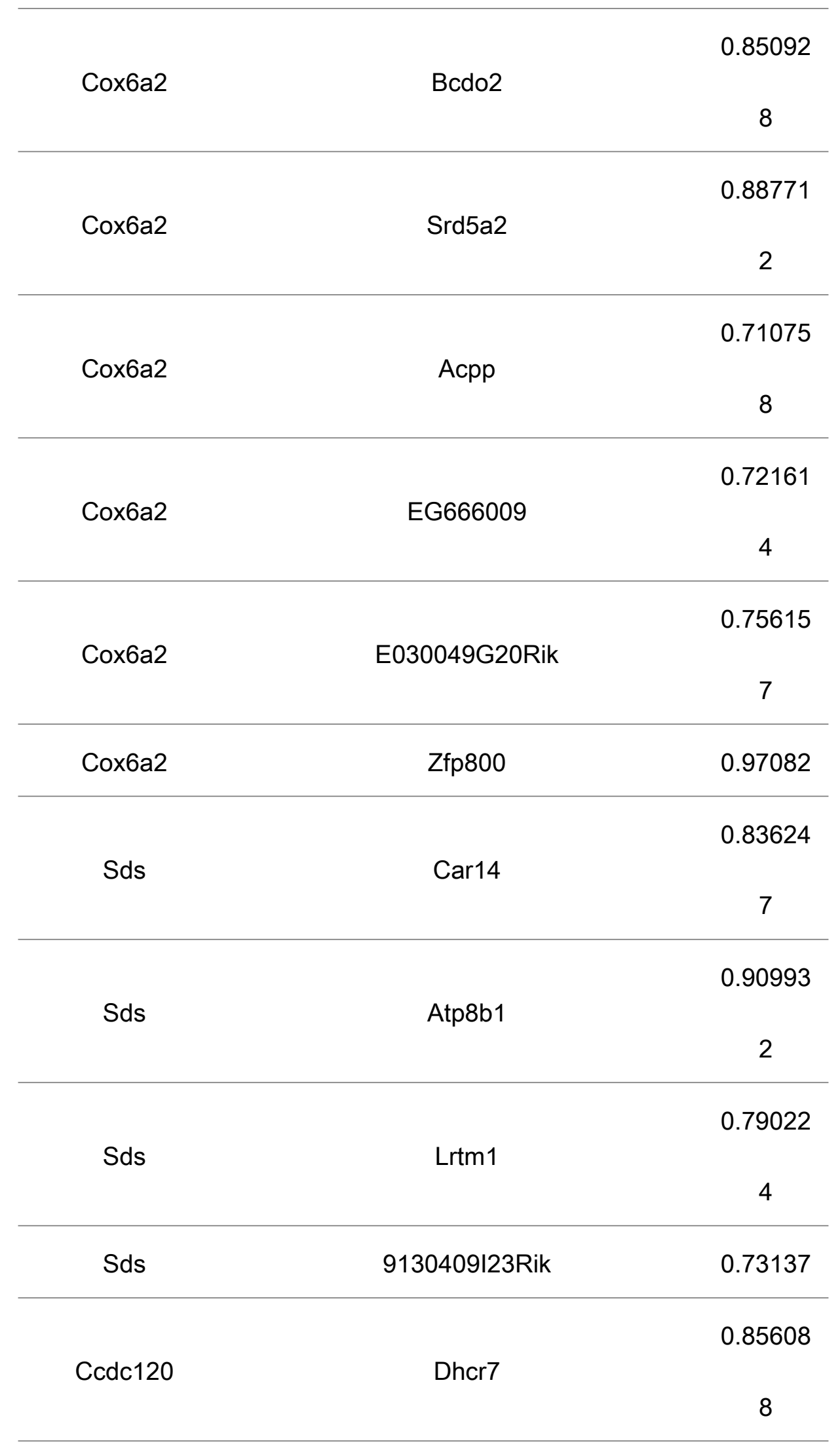




\begin{tabular}{|c|c|c|}
\hline \multirow[t]{2}{*}{ Ccdc120 } & Gpr146 & $\begin{array}{c}0.82630 \\
3\end{array}$ \\
\hline & & 0.84961 \\
\hline \multirow[t]{3}{*}{ Ccdc120 } & Asns & \\
\hline & & 1 \\
\hline & & 0.84955 \\
\hline \multirow[t]{3}{*}{ Ccdc120 } & Abca8a & \\
\hline & & 6 \\
\hline & & 0.80645 \\
\hline \multirow[t]{3}{*}{ Ccdc120 } & Hpgd & \\
\hline & & 4 \\
\hline & & 0.85865 \\
\hline \multirow[t]{3}{*}{ Ccdc120 } & Mrpl49 & \\
\hline & & 8 \\
\hline & & 0.84749 \\
\hline \multirow[t]{3}{*}{ Ccdc120 } & Slc25a42 & \\
\hline & & 5 \\
\hline & & 0.75383 \\
\hline \multirow[t]{3}{*}{ Ccdc120 } & Pmm1 & \\
\hline & & 4 \\
\hline & & 0.92337 \\
\hline \multirow[t]{3}{*}{ Ccdc120 } & Hsd3b5 & \\
\hline & & 3 \\
\hline & & 0.91154 \\
\hline \multirow[t]{3}{*}{ Ccdc120 } & Aox3 & \\
\hline & & 4 \\
\hline & & 0.88002 \\
\hline \multirow[t]{2}{*}{ Ccdc120 } & Smox & \\
\hline & & 5 \\
\hline
\end{tabular}




\begin{tabular}{|c|c|c|}
\hline \multirow[t]{2}{*}{ Ccdc120 } & LOC433886 & $\begin{array}{c}0.77473 \\
7\end{array}$ \\
\hline & & 0.81563 \\
\hline \multirow[t]{3}{*}{ Ccdc120 } & Fn3k & \\
\hline & & 6 \\
\hline & & 0.93312 \\
\hline \multirow[t]{3}{*}{ Ccdc120 } & Ngef & \\
\hline & & 6 \\
\hline & & 0.89869 \\
\hline \multirow[t]{3}{*}{ Ccdc120 } & Zfp750 & \\
\hline & & 8 \\
\hline & & 0.74081 \\
\hline \multirow[t]{3}{*}{ Ccdc120 } & 1810020C02Rik & \\
\hline & & 2 \\
\hline & & 0.81613 \\
\hline \multirow[t]{3}{*}{ Ccdc120 } & Slc41a3 & \\
\hline & & 5 \\
\hline & & 0.79473 \\
\hline \multirow[t]{3}{*}{ Ccdc120 } & Sel1h & \\
\hline & & 5 \\
\hline & & 0.89036 \\
\hline \multirow[t]{3}{*}{ Ccdc120 } & Fam129a & \\
\hline & & 8 \\
\hline & & 0.72656 \\
\hline \multirow[t]{3}{*}{ Ccdc120 } & Ica1 & \\
\hline & & 3 \\
\hline & & 0.74189 \\
\hline \multirow[t]{2}{*}{ Ccdc120 } & B430005K18Rik & \\
\hline & & 8 \\
\hline
\end{tabular}




\begin{tabular}{|c|c|c|}
\hline \multirow[t]{2}{*}{ Ccdc120 } & Gas7 & $\begin{array}{c}0.71898 \\
2\end{array}$ \\
\hline & & 0.85890 \\
\hline \multirow[t]{3}{*}{ Ccdc120 } & Ces1 & \\
\hline & & 1 \\
\hline & & 0.73361 \\
\hline \multirow[t]{3}{*}{ Ccdc120 } & 4833403D03Rik & \\
\hline & & 2 \\
\hline & & 0.87116 \\
\hline \multirow[t]{2}{*}{ Ccdc120 } & Bves & \\
\hline & & 9 \\
\hline \multirow[t]{2}{*}{ Ccdc120 } & D11Lgp2e & 0.72968 \\
\hline & & 0.88147 \\
\hline \multirow[t]{3}{*}{ Ccdc120 } & Bcdo2 & \\
\hline & & 4 \\
\hline & & 0.88323 \\
\hline \multirow[t]{2}{*}{ Ccdc120 } & 5730410E15Rik & \\
\hline & & 8 \\
\hline \multirow[t]{2}{*}{ Car14 } & Atp8b1 & 0.83844 \\
\hline & & 0.73905 \\
\hline \multirow[t]{3}{*}{ Car14 } & Lrtm1 & \\
\hline & & 2 \\
\hline & & 0.84281 \\
\hline \multirow[t]{3}{*}{ Car14 } & 9130409I23Rik & \\
\hline & & 9 \\
\hline & & 0.90362 \\
\hline \multirow[t]{2}{*}{ Dhcr7 } & Asns & \\
\hline & & 9 \\
\hline
\end{tabular}




\begin{tabular}{|c|c|c|}
\hline Dhcr7 & Abca8a & $\begin{array}{c}0.95317 \\
6\end{array}$ \\
\hline Dhcr7 & Hpgd & $\begin{array}{c}0.70499 \\
2\end{array}$ \\
\hline Dhcr7 & Mrpl49 & $\begin{array}{c}0.78725 \\
8\end{array}$ \\
\hline Dhcr7 & 3010026009Rik & $\begin{array}{c}0.86889 \\
3\end{array}$ \\
\hline Dhcr7 & Paqr7 & $\begin{array}{c}0.75252 \\
5\end{array}$ \\
\hline Dhcr7 & Hsd3b5 & $\begin{array}{c}0.70505 \\
7\end{array}$ \\
\hline Dhcr7 & Aox3 & $\begin{array}{c}0.94802 \\
1\end{array}$ \\
\hline Dhcr7 & Smox & $\begin{array}{c}0.79712 \\
6\end{array}$ \\
\hline Dhcr7 & LOC433886 & $\begin{array}{c}0.82400 \\
4\end{array}$ \\
\hline Dhcr7 & Fn3k & $\begin{array}{c}0.71943 \\
2\end{array}$ \\
\hline Dhcr7 & Ngef & 0.89492 \\
\hline
\end{tabular}




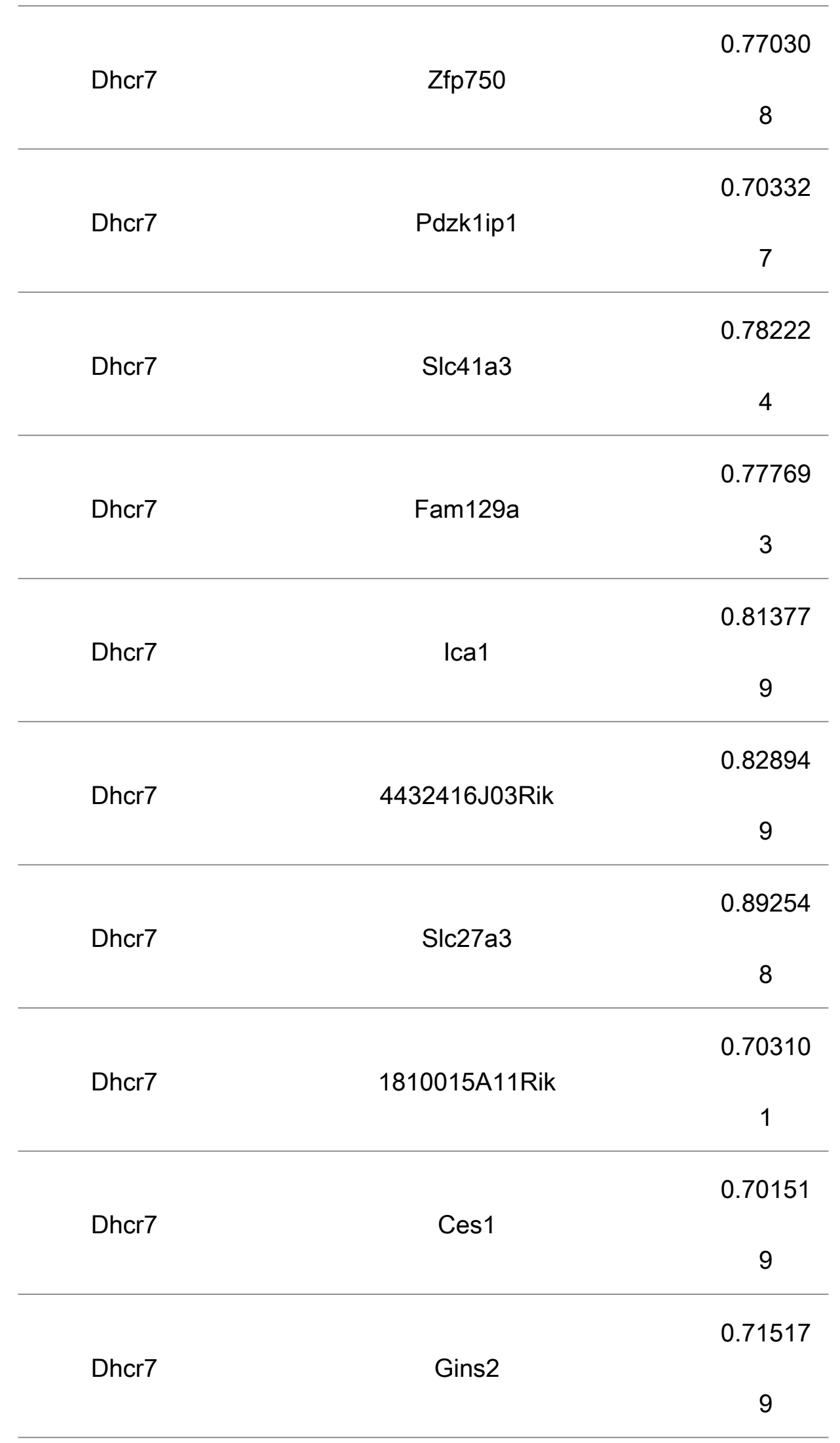




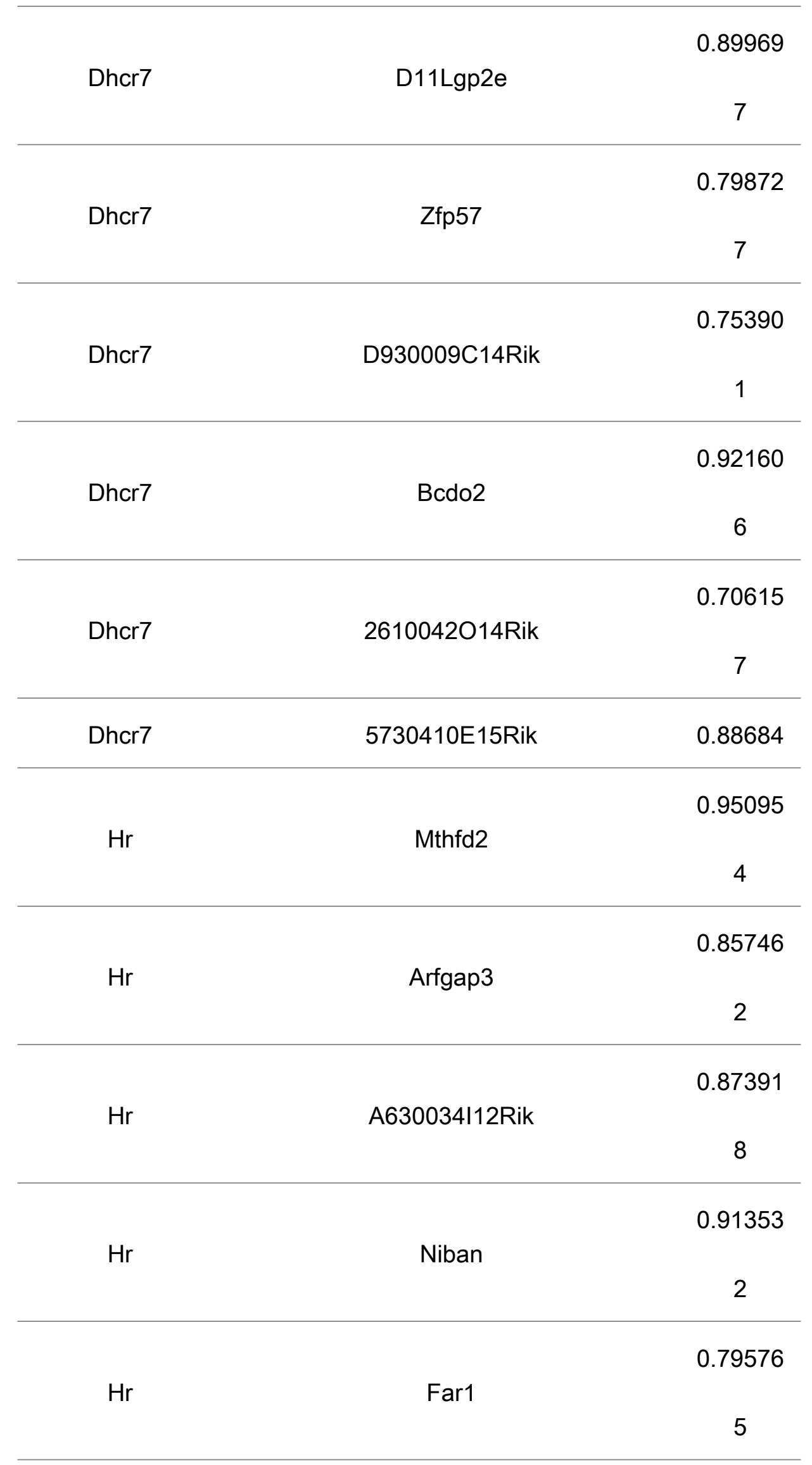




\begin{tabular}{|c|c|c|}
\hline $\mathrm{Hr}$ & Bves & $\begin{array}{c}0.85655 \\
2\end{array}$ \\
\hline & & 0.76054 \\
\hline \multirow[t]{3}{*}{$\mathrm{Hr}$} & 5730410E15Rik & \\
\hline & & 8 \\
\hline & & 0.88426 \\
\hline \multirow[t]{3}{*}{$\mathrm{Hr}$} & 5830411E10Rik & \\
\hline & & 2 \\
\hline & & 0.77698 \\
\hline \multirow[t]{3}{*}{ Gadd45a } & Asns & \\
\hline & & 4 \\
\hline & & 0.88722 \\
\hline \multirow[t]{3}{*}{ Gadd45a } & Hpgd & \\
\hline & & 9 \\
\hline & & 0.71038 \\
\hline \multirow[t]{3}{*}{ Gadd45a } & Mrpl49 & \\
\hline & & 4 \\
\hline & & 0.73182 \\
\hline \multirow[t]{3}{*}{ Gadd45a } & Ngef & \\
\hline & & 9 \\
\hline & & 0.72465 \\
\hline \multirow[t]{3}{*}{ Gadd45a } & Zfp750 & \\
\hline & & 5 \\
\hline & & 0.82210 \\
\hline \multirow[t]{3}{*}{ Gadd45a } & Sel1h & \\
\hline & & 3 \\
\hline & & 0.77477 \\
\hline \multirow[t]{2}{*}{ Gadd45a } & Niban & \\
\hline & & 9 \\
\hline
\end{tabular}




\begin{tabular}{|c|c|c|}
\hline \multirow[t]{2}{*}{ Gadd45a } & Fam129a & $\begin{array}{c}0.73366 \\
2\end{array}$ \\
\hline & & 0.76342 \\
\hline \multirow[t]{3}{*}{ Gadd45a } & Ica1 & \\
\hline & & 8 \\
\hline & & 0.75479 \\
\hline \multirow[t]{2}{*}{ Gadd45a } & LOC242997 & \\
\hline & & 7 \\
\hline \multirow[t]{2}{*}{ Gadd45a } & Zbtb32 & 0.73326 \\
\hline & & 0.75102 \\
\hline \multirow[t]{3}{*}{ Gadd45a } & 1810015A11Rik & \\
\hline & & 1 \\
\hline & & 0.87742 \\
\hline \multirow[t]{3}{*}{ Gadd45a } & D930009C14Rik & \\
\hline & & 1 \\
\hline & & 0.72063 \\
\hline \multirow[t]{3}{*}{ Gadd45a } & Rgs12 & \\
\hline & & 4 \\
\hline & & 0.87563 \\
\hline \multirow[t]{3}{*}{ Gadd45a } & 1810008K04Rik & \\
\hline & & 2 \\
\hline & & 0.74081 \\
\hline \multirow[t]{3}{*}{ Gadd45a } & Ranbp3l & \\
\hline & & 4 \\
\hline & & 0.75707 \\
\hline \multirow[t]{3}{*}{ Spata2L } & Lrtm1 & \\
\hline & & 5 \\
\hline & & 0.76984 \\
\hline \multirow[t]{2}{*}{ Spata2L } & Prlr & \\
\hline & & 2 \\
\hline
\end{tabular}




\begin{tabular}{|c|c|c|}
\hline Spata2L & 1700012H05Rik & 0.77015 \\
\hline & & 0.71145 \\
\hline \multirow[t]{3}{*}{ Tmie } & 3010026009Rik & \\
\hline & & 5 \\
\hline & & 0.80590 \\
\hline \multirow[t]{3}{*}{ Tmie } & LOC100047427 & \\
\hline & & 6 \\
\hline & & 0.81079 \\
\hline \multirow[t]{3}{*}{ Tmie } & Cpsf4l & \\
\hline & & 3 \\
\hline & & 0.88706 \\
\hline \multirow[t]{3}{*}{ Tmie } & Cdc14b & \\
\hline & & 8 \\
\hline & & 0.92568 \\
\hline \multirow[t]{3}{*}{ Tmie } & EG240549 & \\
\hline & & 2 \\
\hline & & 0.84845 \\
\hline \multirow[t]{3}{*}{ Tmie } & 2610528J11Rik & \\
\hline & & 7 \\
\hline & & 0.73997 \\
\hline \multirow[t]{3}{*}{ Tmie } & 1600016N20Rik & \\
\hline & & 6 \\
\hline & & 0.89302 \\
\hline \multirow[t]{3}{*}{ LOC100044164 } & Serpina4-ps1 & \\
\hline & & 8 \\
\hline & & 0.74421 \\
\hline \multirow[t]{3}{*}{ LOC100044164 } & Clstn3 & \\
\hline & & 1 \\
\hline & & 0.70455 \\
\hline \multirow[t]{2}{*}{ LOC100044164 } & Mapk15 & \\
\hline & & 5 \\
\hline
\end{tabular}




\begin{tabular}{|c|c|c|}
\hline Mthfd2 & A630034I12Rik & $\begin{array}{c}0.97051 \\
8\end{array}$ \\
\hline & & 0.89851 \\
\hline \multirow[t]{2}{*}{ Mthfd2 } & Niban & \\
\hline & & 5 \\
\hline \multirow[t]{2}{*}{ Mthfd2 } & Bves & 0.74546 \\
\hline & & 0.86087 \\
\hline \multirow[t]{3}{*}{ Mthfd2 } & 5830411E10Rik & \\
\hline & & 4 \\
\hline & & 0.96200 \\
\hline \multirow[t]{3}{*}{ Mist1 } & Bhlhb8 & \\
\hline & & 3 \\
\hline & & 0.85840 \\
\hline \multirow[t]{3}{*}{ Mist1 } & Slc25a42 & \\
\hline & & 7 \\
\hline & & 0.79035 \\
\hline \multirow[t]{2}{*}{ Mist1 } & Pmm1 & \\
\hline & & 3 \\
\hline \multirow[t]{2}{*}{ Mist1 } & LOC433886 & 0.8496 \\
\hline & & 0.74236 \\
\hline \multirow[t]{3}{*}{ Mist1 } & Slc41a3 & \\
\hline & & 8 \\
\hline & & 0.76813 \\
\hline \multirow[t]{3}{*}{ Mist1 } & AW011738 & \\
\hline & & 5 \\
\hline & & 0.85179 \\
\hline \multirow[t]{2}{*}{ Mist1 } & Isg20 & \\
\hline & & 9 \\
\hline
\end{tabular}




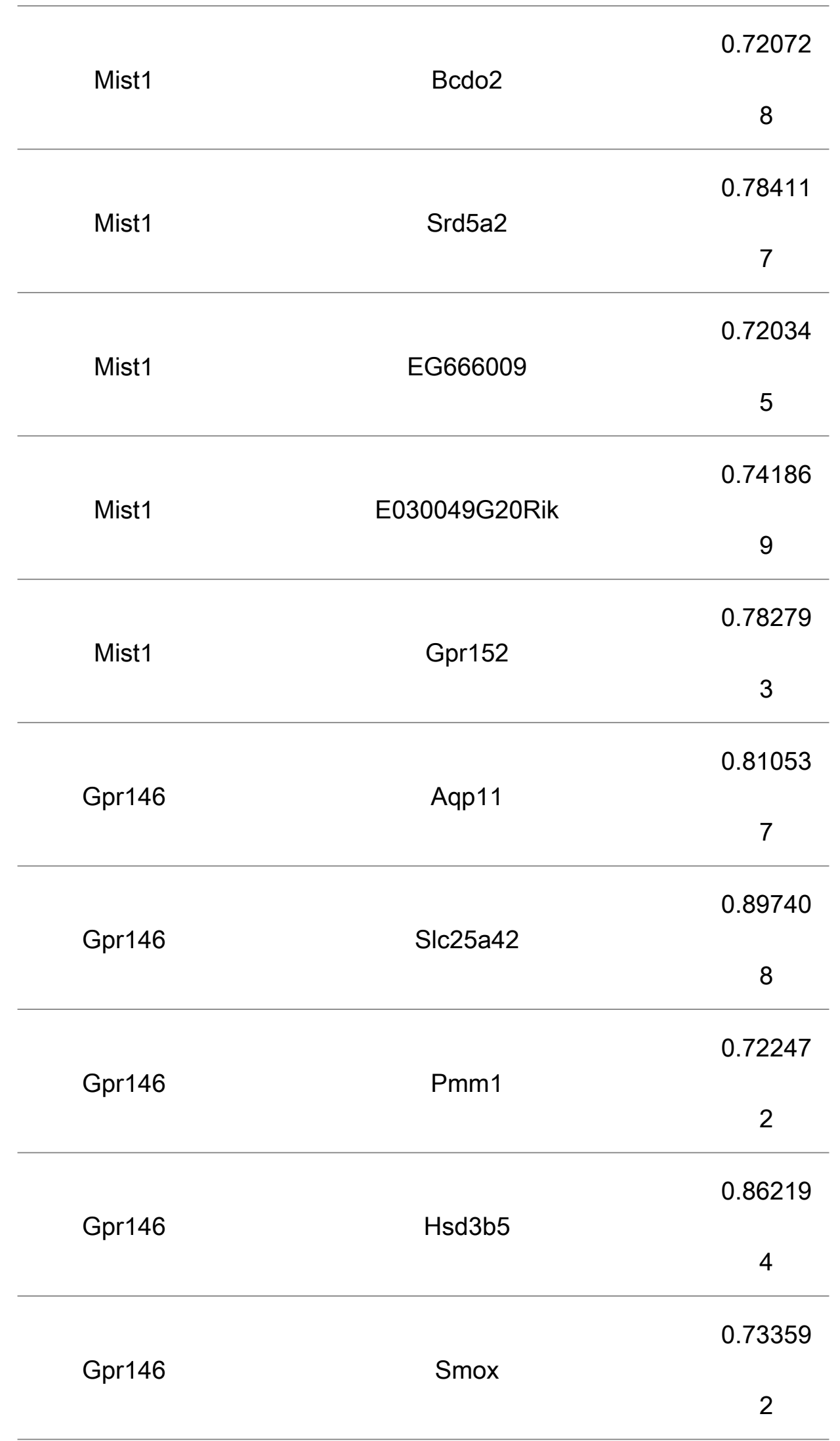




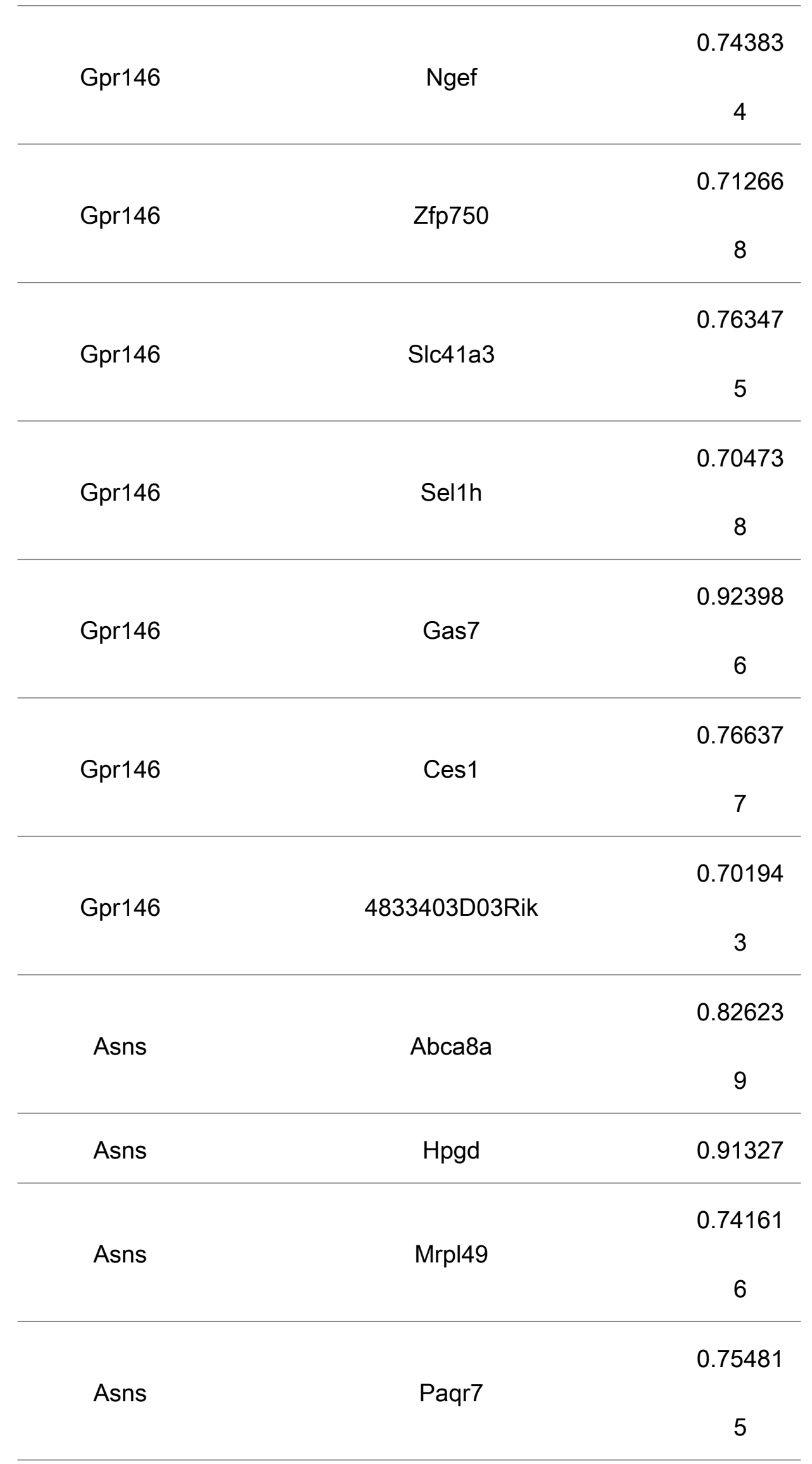




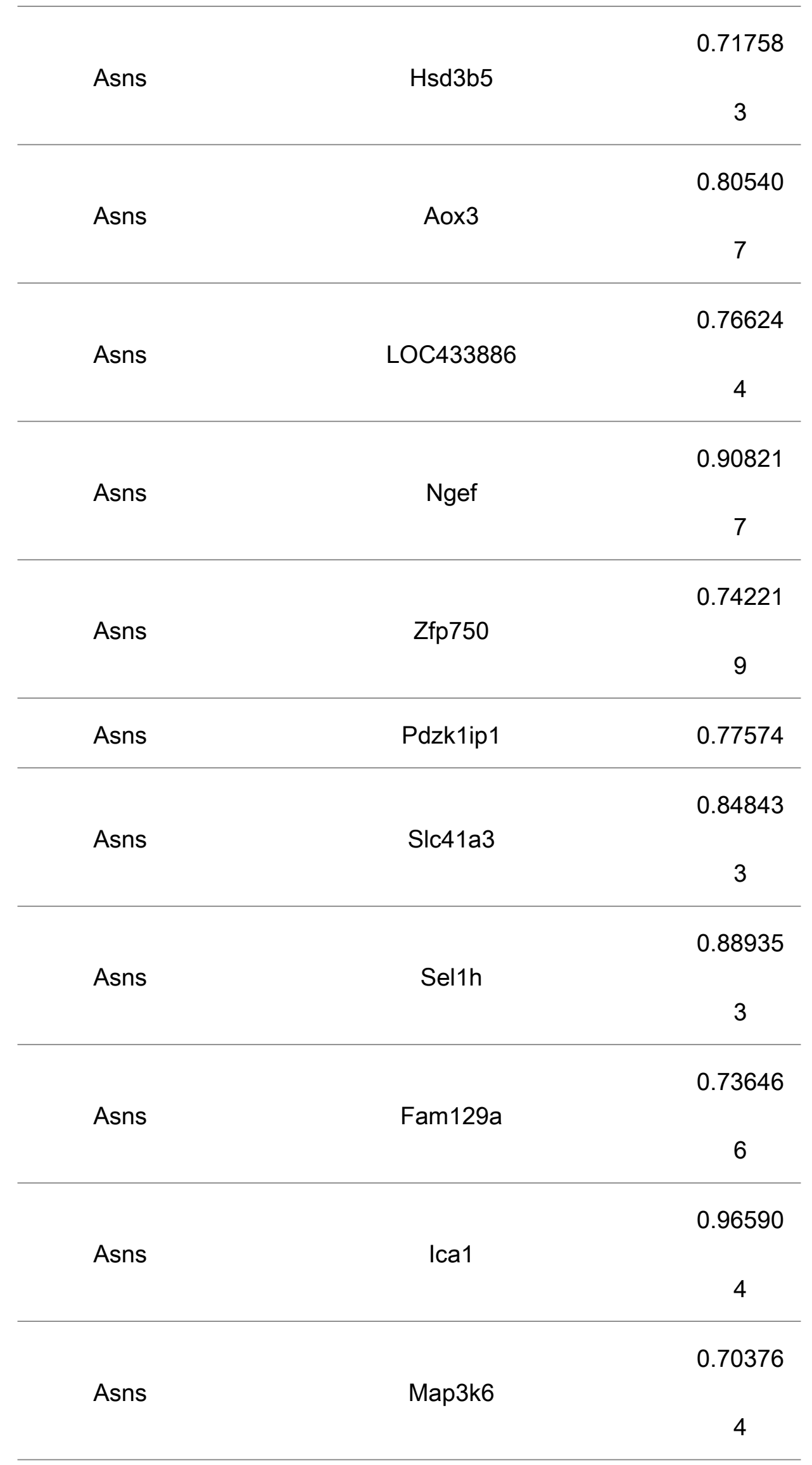




\begin{tabular}{|c|c|c|}
\hline Asns & 4432416J03Rik & 0.87322 \\
\hline & & 0.77738 \\
\hline \multirow[t]{3}{*}{ Asns } & LOC242997 & \\
\hline & & 8 \\
\hline & & 0.73376 \\
\hline \multirow[t]{3}{*}{ Asns } & Zbtb32 & \\
\hline & & 7 \\
\hline & & 0.88859 \\
\hline \multirow[t]{3}{*}{ Asns } & Slc27a3 & \\
\hline & & 7 \\
\hline & & 0.85209 \\
\hline \multirow[t]{3}{*}{ Asns } & 1810015A11Rik & \\
\hline & & 2 \\
\hline & & 0.75035 \\
\hline \multirow[t]{3}{*}{ Asns } & Gins2 & \\
\hline & & 5 \\
\hline & & 0.83496 \\
\hline \multirow[t]{3}{*}{ Asns } & D11Lgp2e & \\
\hline & & 8 \\
\hline & & 0.87501 \\
\hline \multirow[t]{3}{*}{ Asns } & D930009C14Rik & \\
\hline & & 3 \\
\hline & & 0.78524 \\
\hline \multirow[t]{3}{*}{ Asns } & Bcdo2 & \\
\hline & & 3 \\
\hline & & 0.78115 \\
\hline \multirow[t]{3}{*}{ Asns } & 5730410E15Rik & \\
\hline & & 1 \\
\hline & & 0.72047 \\
\hline \multirow[t]{2}{*}{ Asns } & B230207L18Rik & \\
\hline & & 2 \\
\hline
\end{tabular}




\begin{tabular}{|c|c|c|}
\hline \multirow[t]{2}{*}{ Abca8a } & Mrpl49 & $\begin{array}{c}0.87624 \\
2\end{array}$ \\
\hline & & 0.77555 \\
\hline \multirow[t]{3}{*}{ Abca8a } & 3010026009Rik & \\
\hline & & 5 \\
\hline & & 0.74877 \\
\hline \multirow[t]{3}{*}{ Abca8a } & Paqr7 & \\
\hline & & 8 \\
\hline & & 0.74561 \\
\hline \multirow[t]{3}{*}{ Abca8a } & Hsd3b5 & \\
\hline & & 4 \\
\hline & & 0.96452 \\
\hline \multirow[t]{3}{*}{ Abca8a } & Aox3 & \\
\hline & & 8 \\
\hline & & 0.75162 \\
\hline \multirow[t]{3}{*}{ Abca8a } & Smox & \\
\hline & & 6 \\
\hline & & 0.73103 \\
\hline \multirow[t]{3}{*}{ Abca8a } & LOC433886 & \\
\hline & & 1 \\
\hline & & 0.81386 \\
\hline \multirow[t]{3}{*}{ Abca8a } & Fn3k & \\
\hline & & 4 \\
\hline & & 0.87458 \\
\hline \multirow[t]{3}{*}{ Abca8a } & Ngef & \\
\hline & & 5 \\
\hline & & 0.85111 \\
\hline \multirow[t]{2}{*}{ Abca8a } & Zfp750 & \\
\hline & & 6 \\
\hline
\end{tabular}




\begin{tabular}{|c|c|c|}
\hline \multirow[t]{2}{*}{ Abca8a } & Hacl1 & $\begin{array}{c}0.74080 \\
5\end{array}$ \\
\hline & & 0.87754 \\
\hline \multirow[t]{3}{*}{ Abca8a } & Fam129a & \\
\hline & & 4 \\
\hline & & 0.72180 \\
\hline \multirow[t]{2}{*}{ Abca8a } & Ica1 & \\
\hline & & 2 \\
\hline \multirow[t]{2}{*}{ Abca8a } & Slc27a3 & 0.85159 \\
\hline & & 0.76332 \\
\hline \multirow[t]{3}{*}{ Abca8a } & Ces1 & \\
\hline & & 1 \\
\hline & & 0.71441 \\
\hline \multirow[t]{3}{*}{ Abca8a } & Grb14 & \\
\hline & & 1 \\
\hline & & 0.75362 \\
\hline \multirow[t]{3}{*}{ Abca8a } & D11Lgp2e & \\
\hline & & 1 \\
\hline & & 0.79775 \\
\hline \multirow[t]{3}{*}{ Abca8a } & Zfp57 & \\
\hline & & 8 \\
\hline & & 0.76377 \\
\hline \multirow[t]{2}{*}{ Abca8a } & D930009C14Rik & \\
\hline & & 3 \\
\hline Abca8a & Bcdo2 & 0.85227 \\
\hline \multirow[t]{2}{*}{ Abca8a } & 5730410E15Rik & 0.90264 \\
\hline & & 0.79810 \\
\hline \multirow[t]{2}{*}{ Abca8a } & Nphp3 & \\
\hline & & 9 \\
\hline
\end{tabular}




\begin{tabular}{|c|c|c|}
\hline Abca8a & LOC381738 & $\begin{array}{c}0.72282 \\
3\end{array}$ \\
\hline Abca8a & E030049G20Rik & $\begin{array}{c}0.71361 \\
3\end{array}$ \\
\hline Abca8a & Ranbp3l & $\begin{array}{c}0.72462 \\
7\end{array}$ \\
\hline Hpgd & Hsd3b5 & $\begin{array}{c}0.73069 \\
6\end{array}$ \\
\hline Hpgd & Ngef & $\begin{array}{c}0.82712 \\
2\end{array}$ \\
\hline Hpgd & Zfp750 & $\begin{array}{c}0.70198 \\
6\end{array}$ \\
\hline Hpgd & Slc41a3 & $\begin{array}{c}0.78527 \\
5\end{array}$ \\
\hline Hpgd & Sel1h & $\begin{array}{c}0.98896 \\
8\end{array}$ \\
\hline Hpgd & Ica1 & $\begin{array}{c}0.89436 \\
3\end{array}$ \\
\hline Hpgd & 4432416J03Rik & $\begin{array}{c}0.71290 \\
6\end{array}$ \\
\hline Hpgd & LOC242997 & 0.90006 \\
\hline
\end{tabular}




\begin{tabular}{|c|c|c|}
\hline Hpgd & Zbtb32 & 0.78512 \\
\hline & & 0.78434 \\
\hline \multirow[t]{3}{*}{ Hpgd } & 1810015A11Rik & \\
\hline & & 9 \\
\hline & & 0.82340 \\
\hline \multirow[t]{3}{*}{ Hpgd } & D930009C14Rik & \\
\hline & & 5 \\
\hline & & 0.79709 \\
\hline \multirow[t]{3}{*}{ Arfgap3 } & Niban & \\
\hline & & 2 \\
\hline & & 0.79394 \\
\hline \multirow[t]{2}{*}{ Arfgap3 } & Far1 & \\
\hline & & 2 \\
\hline Arfgap3 & Guca2a & 0.76849 \\
\hline \multirow[t]{2}{*}{ Arfgap3 } & Bves & 0.84365 \\
\hline & & 0.84048 \\
\hline \multirow[t]{3}{*}{ Arfgap3 } & 5730410E15Rik & \\
\hline & & 7 \\
\hline & & 0.74152 \\
\hline \multirow[t]{3}{*}{ Arfgap3 } & LOC381738 & \\
\hline & & 2 \\
\hline & & 0.79475 \\
\hline \multirow[t]{3}{*}{ Arfgap3 } & 5830411E10Rik & \\
\hline & & 2 \\
\hline & & 0.72348 \\
\hline \multirow[t]{3}{*}{ Arfgap3 } & Ranbp3l & \\
\hline & & 8 \\
\hline & & 0.73826 \\
\hline \multirow[t]{2}{*}{ Bhlhb8 } & Slc25a42 & \\
\hline & & 7 \\
\hline
\end{tabular}




\begin{tabular}{|c|c|c|}
\hline \multirow[t]{2}{*}{ Bhlhb8 } & LOC433886 & $\begin{array}{c}0.80603 \\
9\end{array}$ \\
\hline & & 0.85542 \\
\hline \multirow[t]{3}{*}{ Bhlhb8 } & AW011738 & \\
\hline & & 5 \\
\hline & & 0.92048 \\
\hline \multirow[t]{3}{*}{ Bhlhb8 } & Isg20 & \\
\hline & & 9 \\
\hline & & 0.72966 \\
\hline \multirow[t]{3}{*}{ Bhlhb8 } & Lpin3 & \\
\hline & & 1 \\
\hline & & 0.75839 \\
\hline \multirow[t]{3}{*}{ Bhlhb8 } & Gpr152 & \\
\hline & & 7 \\
\hline & & 0.78157 \\
\hline \multirow[t]{3}{*}{ Dbp } & lqce & \\
\hline & & 1 \\
\hline & & 0.73021 \\
\hline \multirow[t]{3}{*}{ Dbp } & Сyp2c39 & \\
\hline & & 7 \\
\hline & & 0.95255 \\
\hline \multirow[t]{3}{*}{ D0H4S114 } & 9030024J15Rik & \\
\hline & & 4 \\
\hline & & 0.91682 \\
\hline \multirow[t]{3}{*}{ Mrpl49 } & Hsd3b5 & \\
\hline & & 9 \\
\hline & & 0.83959 \\
\hline \multirow[t]{2}{*}{ Mrpl49 } & Aox3 & \\
\hline & & 4 \\
\hline
\end{tabular}




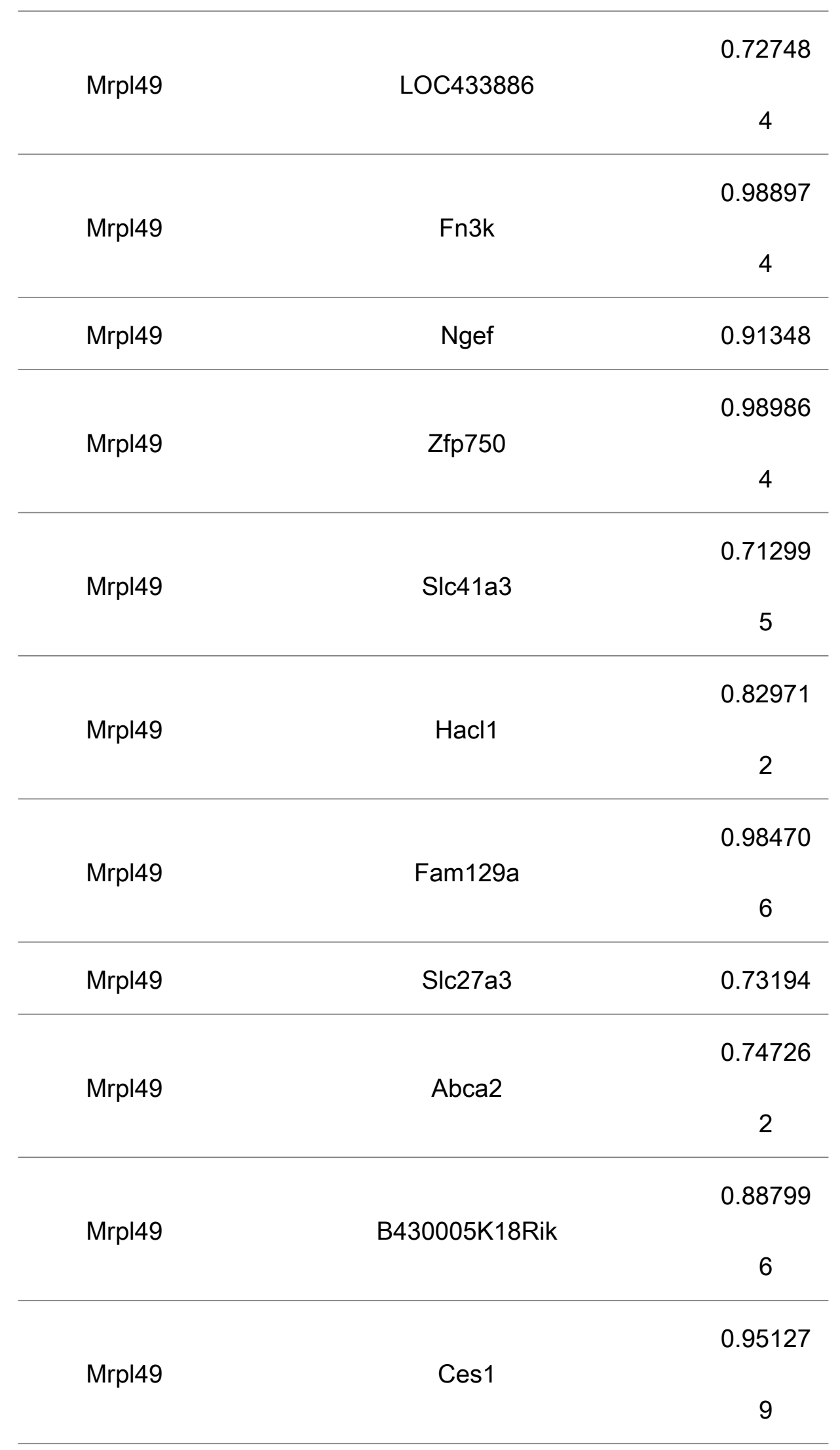




\begin{tabular}{|c|c|c|}
\hline Mrpl49 & Grb14 & $\begin{array}{c}0.70181 \\
8\end{array}$ \\
\hline & & 0.73301 \\
\hline \multirow[t]{3}{*}{ Mrpl49 } & Bcdo2 & \\
\hline & & 8 \\
\hline & & 0.85137 \\
\hline \multirow[t]{3}{*}{ Mrpl49 } & Rgs12 & \\
\hline & & 7 \\
\hline & & 0.73290 \\
\hline \multirow[t]{3}{*}{ Mrpl49 } & 5730410E15Rik & \\
\hline & & 8 \\
\hline & & 0.72464 \\
\hline \multirow[t]{3}{*}{ Mrpl49 } & LOC381738 & \\
\hline & & 8 \\
\hline & & 0.70512 \\
\hline \multirow[t]{3}{*}{ Mrpl49 } & E030049G20Rik & \\
\hline & & 6 \\
\hline & & 0.78917 \\
\hline \multirow[t]{2}{*}{ Mrpl49 } & Gpr152 & \\
\hline & & 2 \\
\hline \multirow[t]{2}{*}{ 3010026009Rik } & Aox3 & 0.75623 \\
\hline & & 0.89722 \\
\hline \multirow[t]{3}{*}{ 3010026009Rik } & LOC100047427 & \\
\hline & & 4 \\
\hline & & 0.83420 \\
\hline \multirow[t]{3}{*}{ 3010026009Rik } & Cdc14b & \\
\hline & & 7 \\
\hline & & 0.78145 \\
\hline \multirow{2}{*}{ 3010026O09Rik } & Prss8 & \\
\hline & & 9 \\
\hline
\end{tabular}




\begin{tabular}{|c|c|c|}
\hline 3010026O09Rik & EG240549 & $\begin{array}{c}0.85570 \\
3\end{array}$ \\
\hline 3010026009Rik & 4432416J03Rik & $\begin{array}{c}0.77306 \\
1\end{array}$ \\
\hline 3010026O09Rik & Slc27a3 & $\begin{array}{c}0.79034 \\
4\end{array}$ \\
\hline 3010026O09Rik & D11Lgp2e & $\begin{array}{c}0.84696 \\
1\end{array}$ \\
\hline 3010026009Rik & Zfp57 & $\begin{array}{c}0.84908 \\
8\end{array}$ \\
\hline 3010026009Rik & Bcdo2 & $\begin{array}{c}0.76741 \\
7\end{array}$ \\
\hline 3010026O09Rik & 2610042O14Rik & $\begin{array}{c}0.74062 \\
8\end{array}$ \\
\hline 3010026009Rik & 5730410E15Rik & 0.70304 \\
\hline Kdelr3 & Bcmo1 & $\begin{array}{c}0.85651 \\
4\end{array}$ \\
\hline Kdelr3 & Smox & $\begin{array}{c}0.74102 \\
1\end{array}$ \\
\hline Kdelr3 & 1810020C02Rik & $\begin{array}{c}0.86888 \\
7\end{array}$ \\
\hline
\end{tabular}




\begin{tabular}{|c|c|c|}
\hline \multirow[t]{2}{*}{ Kdelr3 } & Ttc39a & $\begin{array}{c}0.71573 \\
8\end{array}$ \\
\hline & & 0.91774 \\
\hline \multirow[t]{3}{*}{ Kdelr3 } & Mcf2I & \\
\hline & & 4 \\
\hline & & 0.77603 \\
\hline \multirow[t]{3}{*}{ Kdelr3 } & LOC219106 & \\
\hline & & 6 \\
\hline & & 0.74600 \\
\hline \multirow[t]{3}{*}{ Kdelr3 } & Guca2a & \\
\hline & & 3 \\
\hline & & 0.75223 \\
\hline \multirow[t]{3}{*}{ Kdelr3 } & 4833403D03Rik & \\
\hline & & 9 \\
\hline & & 0.75862 \\
\hline \multirow[t]{3}{*}{ Kdelr3 } & Srd5a2 & \\
\hline & & 2 \\
\hline & & 0.81225 \\
\hline \multirow[t]{3}{*}{ Kdelr3 } & St6galnac4 & \\
\hline & & 6 \\
\hline & & 0.82302 \\
\hline \multirow[t]{3}{*}{ Kdelr3 } & Zfp800 & \\
\hline & & 7 \\
\hline & & 0.75355 \\
\hline \multirow[t]{2}{*}{ Aqp11 } & Hsd3b5 & \\
\hline & & 4 \\
\hline \multirow[t]{2}{*}{ Aqp11 } & Slc25a23 & 0.7239 \\
\hline & & 0.71342 \\
\hline \multirow[t]{2}{*}{ Aqp11 } & Bik & \\
\hline & & 8 \\
\hline
\end{tabular}




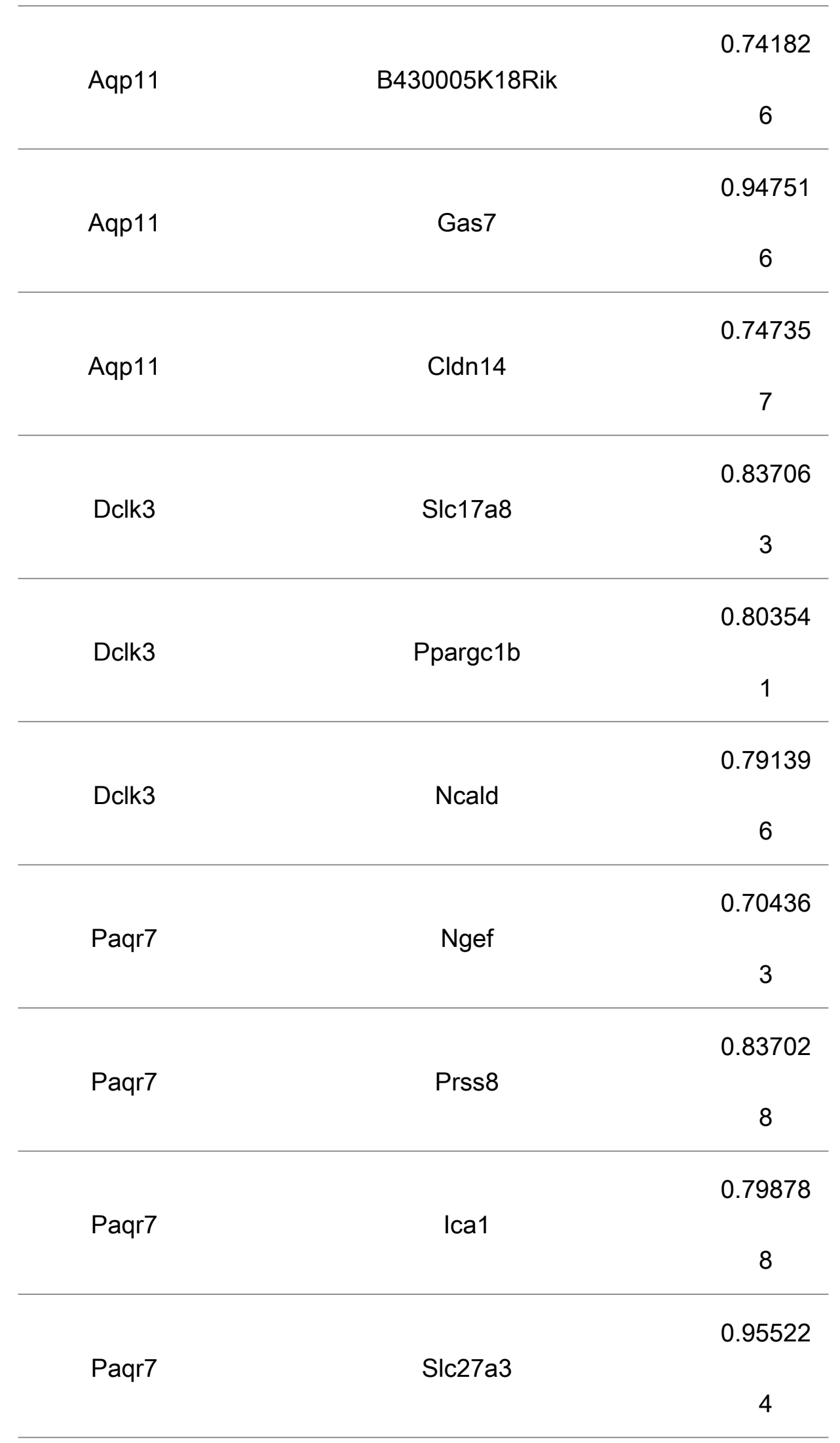




\begin{tabular}{|c|c|c|}
\hline \multirow[t]{2}{*}{ Paqr7 } & 1810015A11Rik & $\begin{array}{c}0.73580 \\
3\end{array}$ \\
\hline & & 0.78525 \\
\hline \multirow[t]{3}{*}{ Paqr7 } & Gins2 & \\
\hline & & 7 \\
\hline & & 0.73082 \\
\hline \multirow[t]{3}{*}{ Paqr7 } & Zfp57 & \\
\hline & & 8 \\
\hline & & 0.81252 \\
\hline \multirow[t]{2}{*}{ Paqr7 } & D930009C14Rik & \\
\hline & & 6 \\
\hline \multirow[t]{2}{*}{ Paqr7 } & 1810046K07Rik & 0.9059 \\
\hline & & 0.74647 \\
\hline \multirow[t]{3}{*}{ Bcmo1 } & Cyb5r1 & \\
\hline & & 5 \\
\hline & & 0.89128 \\
\hline \multirow[t]{3}{*}{ Bcmo1 } & Ttc39a & \\
\hline & & 8 \\
\hline & & 0.76169 \\
\hline \multirow[t]{3}{*}{ Bcmo1 } & Mcf2I & \\
\hline & & 1 \\
\hline & & 0.92032 \\
\hline \multirow[t]{3}{*}{ Bcmo1 } & Guca2a & \\
\hline & & 7 \\
\hline & & 0.90352 \\
\hline \multirow[t]{3}{*}{ Slc25a42 } & Pmm1 & \\
\hline & & 3 \\
\hline & & 0.81446 \\
\hline \multirow[t]{2}{*}{ Slc25a42 } & Hsd3b5 & \\
\hline & & 9 \\
\hline
\end{tabular}




\begin{tabular}{|c|c|c|}
\hline Slc25a42 & Aox3 & $\begin{array}{c}0.72565 \\
5\end{array}$ \\
\hline Slc25a42 & Smox & 0.86633 \\
\hline Slc25a42 & LOC433886 & $\begin{array}{c}0.87399 \\
2\end{array}$ \\
\hline Slc25a42 & Ngef & 0.82258 \\
\hline Slc25a42 & Zfp750 & $\begin{array}{c}0.71354 \\
8\end{array}$ \\
\hline Slc25a42 & 1810020C02Rik & $\begin{array}{c}0.80775 \\
3\end{array}$ \\
\hline Slc25a42 & Slc41a3 & $\begin{array}{c}0.88579 \\
4\end{array}$ \\
\hline Slc25a42 & C430002P19Rik & $\begin{array}{c}0.80159 \\
9\end{array}$ \\
\hline Slc25a42 & Ces1 & $\begin{array}{c}0.77728 \\
2\end{array}$ \\
\hline Slc25a42 & D11Lgp2e & 0.74692 \\
\hline Slc25a42 & Bcdo2 & $\begin{array}{c}0.85393 \\
8\end{array}$ \\
\hline Slc25a42 & Gpr152 & $\begin{array}{c}0.75934 \\
1\end{array}$ \\
\hline
\end{tabular}




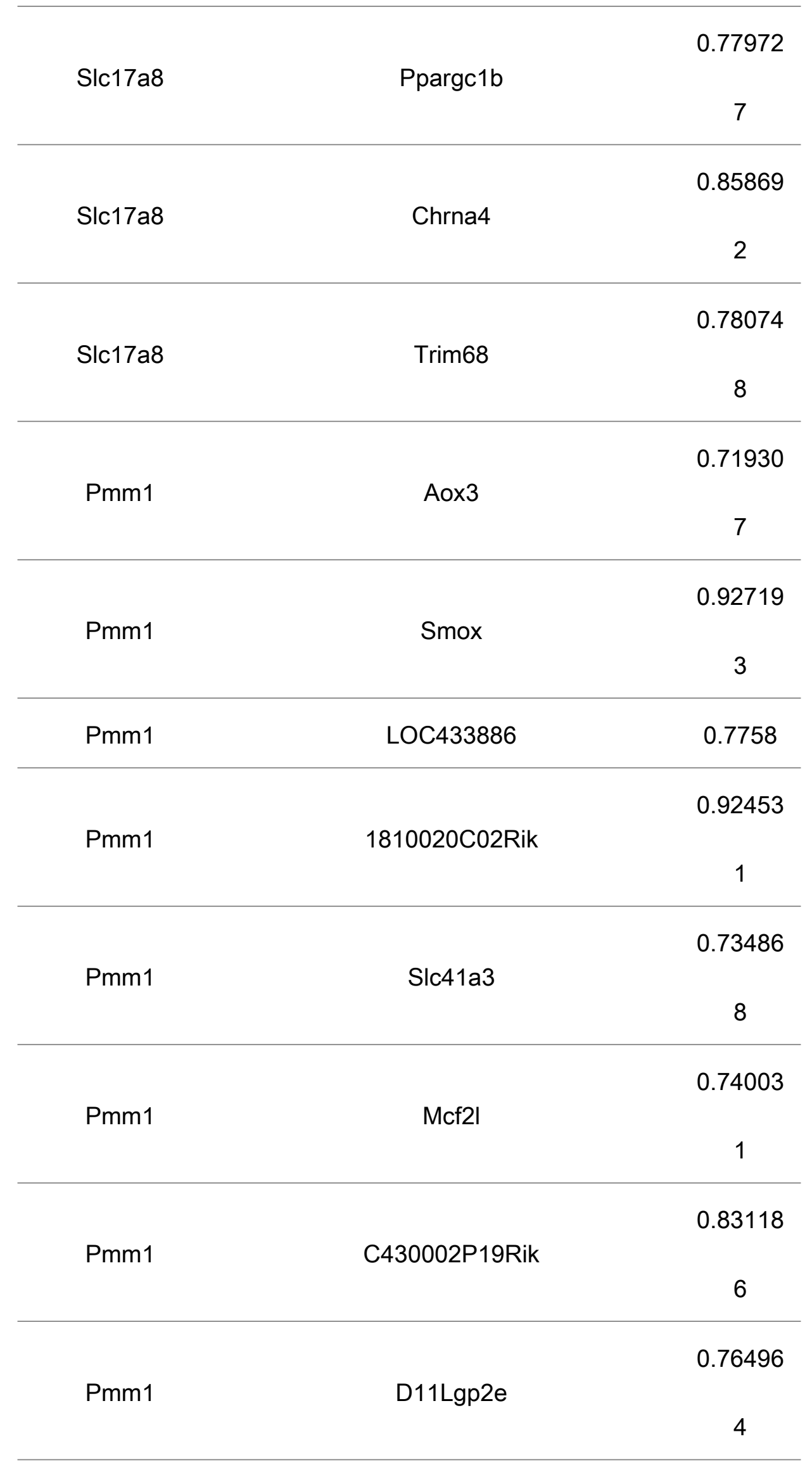




\begin{tabular}{|c|c|c|}
\hline Pmm1 & Ube2u & 0.84224 \\
\hline & & 0.88581 \\
\hline \multirow{3}{*}{ Pmm1 } & Bcdo2 & \\
\hline & & 3 \\
\hline & & 0.73904 \\
\hline \multirow[t]{3}{*}{ Pmm1 } & Srd5a2 & \\
\hline & & 7 \\
\hline & & 0.80324 \\
\hline \multirow[t]{3}{*}{ Pmm1 } & Cnksr1 & \\
\hline & & 1 \\
\hline & & 0.83787 \\
\hline \multirow[t]{3}{*}{ Pmm1 } & EG666009 & \\
\hline & & 6 \\
\hline & & 0.75987 \\
\hline \multirow[t]{3}{*}{ Pmm1 } & Zfp800 & \\
\hline & & 1 \\
\hline & & 0.78226 \\
\hline \multirow[t]{3}{*}{ Hsd3b5 } & Aox3 & \\
\hline & & 6 \\
\hline & & 0.74424 \\
\hline \multirow[t]{3}{*}{ Hsd3b5 } & Smox & \\
\hline & & 5 \\
\hline & & 0.70565 \\
\hline \multirow[t]{3}{*}{ Hsd3b5 } & Slc25a23 & \\
\hline & & 5 \\
\hline & & 0.73135 \\
\hline \multirow[t]{3}{*}{ Hsd3b5 } & LOC433886 & \\
\hline & & 5 \\
\hline & & 0.91810 \\
\hline \multirow[t]{2}{*}{ Hsd3b5 } & Fn3k & \\
\hline & & 9 \\
\hline
\end{tabular}




\begin{tabular}{|c|c|c|}
\hline \multirow[t]{2}{*}{ Hsd3b5 } & Ngef & $\begin{array}{c}0.90323 \\
1\end{array}$ \\
\hline & & 0.95941 \\
\hline \multirow[t]{3}{*}{ Hsd3b5 } & Zfp750 & \\
\hline & & 4 \\
\hline & & 0.72508 \\
\hline \multirow[t]{3}{*}{ Hsd3b5 } & Bik & \\
\hline & & 6 \\
\hline & & 0.77577 \\
\hline \multirow[t]{3}{*}{ Hsd3b5 } & Slc41a3 & \\
\hline & & 6 \\
\hline & & 0.73501 \\
\hline \multirow[t]{3}{*}{ Hsd3b5 } & Hacl1 & \\
\hline & & 8 \\
\hline & & 0.70859 \\
\hline \multirow[t]{3}{*}{ Hsd3b5 } & Sel1h & \\
\hline & & 6 \\
\hline & & 0.92843 \\
\hline \multirow[t]{3}{*}{ Hsd3b5 } & Fam129a & \\
\hline & & 3 \\
\hline & & 0.90900 \\
\hline \multirow[t]{3}{*}{ Hsd3b5 } & B430005K18Rik & \\
\hline & & 2 \\
\hline & & 0.81765 \\
\hline \multirow[t]{3}{*}{ Hsd3b5 } & Gas7 & \\
\hline & & 3 \\
\hline & & 0.96752 \\
\hline \multirow[t]{2}{*}{ Hsd3b5 } & Ces1 & \\
\hline & & 7 \\
\hline
\end{tabular}




\begin{tabular}{|c|c|c|}
\hline \multirow[t]{2}{*}{ Hsd3b5 } & 4833403D03Rik & $\begin{array}{c}0.70145 \\
3\end{array}$ \\
\hline & & 0.72415 \\
\hline \multirow[t]{3}{*}{ Hsd3b5 } & Bves & \\
\hline & & 6 \\
\hline & & 0.73932 \\
\hline \multirow[t]{2}{*}{ Hsd3b5 } & Bcdo2 & \\
\hline & & 7 \\
\hline \multirow[t]{2}{*}{ Hsd3b5 } & Rgs12 & 0.86242 \\
\hline & & 0.70372 \\
\hline \multirow[t]{3}{*}{ Hsd3b5 } & 5730410E15Rik & \\
\hline & & 2 \\
\hline & & 0.76909 \\
\hline \multirow[t]{3}{*}{ Hsd3b5 } & Gpr152 & \\
\hline & & 8 \\
\hline & & 0.88527 \\
\hline \multirow[t]{3}{*}{ Aox3 } & Smox & \\
\hline & & 8 \\
\hline & & 0.76428 \\
\hline \multirow[t]{3}{*}{ Aox3 } & LOC433886 & \\
\hline & & 8 \\
\hline & & 0.78380 \\
\hline \multirow[t]{3}{*}{ Aox3 } & Fn3k & \\
\hline & & 9 \\
\hline & & 0.87346 \\
\hline \multirow[t]{3}{*}{ Aox3 } & Ngef & \\
\hline & & 8 \\
\hline & & 0.84065 \\
\hline \multirow[t]{2}{*}{ Aox3 } & Zfp750 & \\
\hline & & 6 \\
\hline
\end{tabular}




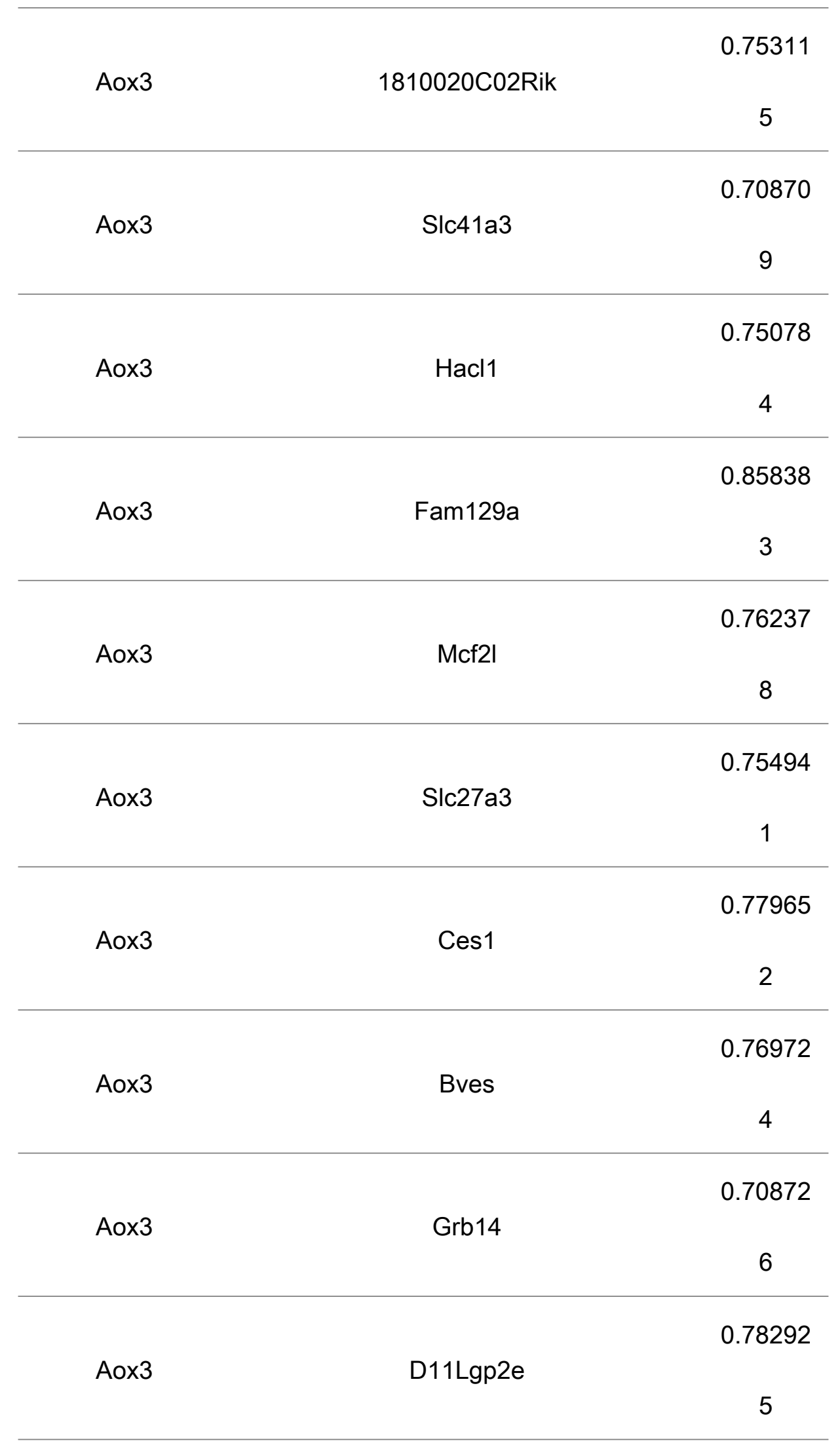




\begin{tabular}{|c|c|c|}
\hline Aox3 & Zfp57 & $\begin{array}{c}0.73473 \\
8\end{array}$ \\
\hline Aox3 & Bcdo2 & $\begin{array}{c}0.93566 \\
2\end{array}$ \\
\hline Aox3 & 5730410E15Rik & $\begin{array}{c}0.95365 \\
8\end{array}$ \\
\hline Aox3 & LOC381738 & $\begin{array}{c}0.71985 \\
8\end{array}$ \\
\hline Aox3 & E030049G20Rik & $\begin{array}{c}0.73128 \\
3\end{array}$ \\
\hline Aox3 & Zfp800 & $\begin{array}{c}0.77140 \\
1\end{array}$ \\
\hline Smox & LOC433886 & $\begin{array}{c}0.75907 \\
5\end{array}$ \\
\hline Smox & Ngef & $\begin{array}{c}0.76801 \\
5\end{array}$ \\
\hline Smox & Zfp750 & 0.70654 \\
\hline Smox & 1810020C02Rik & $\begin{array}{c}0.95779 \\
9\end{array}$ \\
\hline Smox & Slc41a3 & $\begin{array}{c}0.70901 \\
8\end{array}$ \\
\hline
\end{tabular}




\begin{tabular}{|c|c|c|}
\hline \multirow[t]{2}{*}{ Smox } & Mcf2I & $\begin{array}{c}0.86583 \\
4\end{array}$ \\
\hline & & 0.70136 \\
\hline \multirow[t]{3}{*}{ Smox } & C430002P19Rik & \\
\hline & & 6 \\
\hline & & 0.71234 \\
\hline \multirow[t]{3}{*}{ Smox } & Ces1 & \\
\hline & & 3 \\
\hline & & 0.82093 \\
\hline \multirow[t]{3}{*}{ Smox } & 4833403D03Rik & \\
\hline & & 5 \\
\hline & & 0.78191 \\
\hline \multirow[t]{3}{*}{ Smox } & Bves & \\
\hline & & 9 \\
\hline & & 0.74608 \\
\hline \multirow[t]{3}{*}{ Smox } & D11Lgp2e & \\
\hline & & 3 \\
\hline & & 0.71754 \\
\hline \multirow[t]{3}{*}{ Smox } & Ube2u & \\
\hline & & 7 \\
\hline & & 0.94857 \\
\hline \multirow[t]{3}{*}{ Smox } & Bcdo2 & \\
\hline & & 6 \\
\hline & & 0.70117 \\
\hline \multirow[t]{3}{*}{ Smox } & Srd5a2 & \\
\hline & & 4 \\
\hline & & 0.84608 \\
\hline \multirow[t]{2}{*}{ Smox } & 5730410E15Rik & \\
\hline & & 9 \\
\hline
\end{tabular}




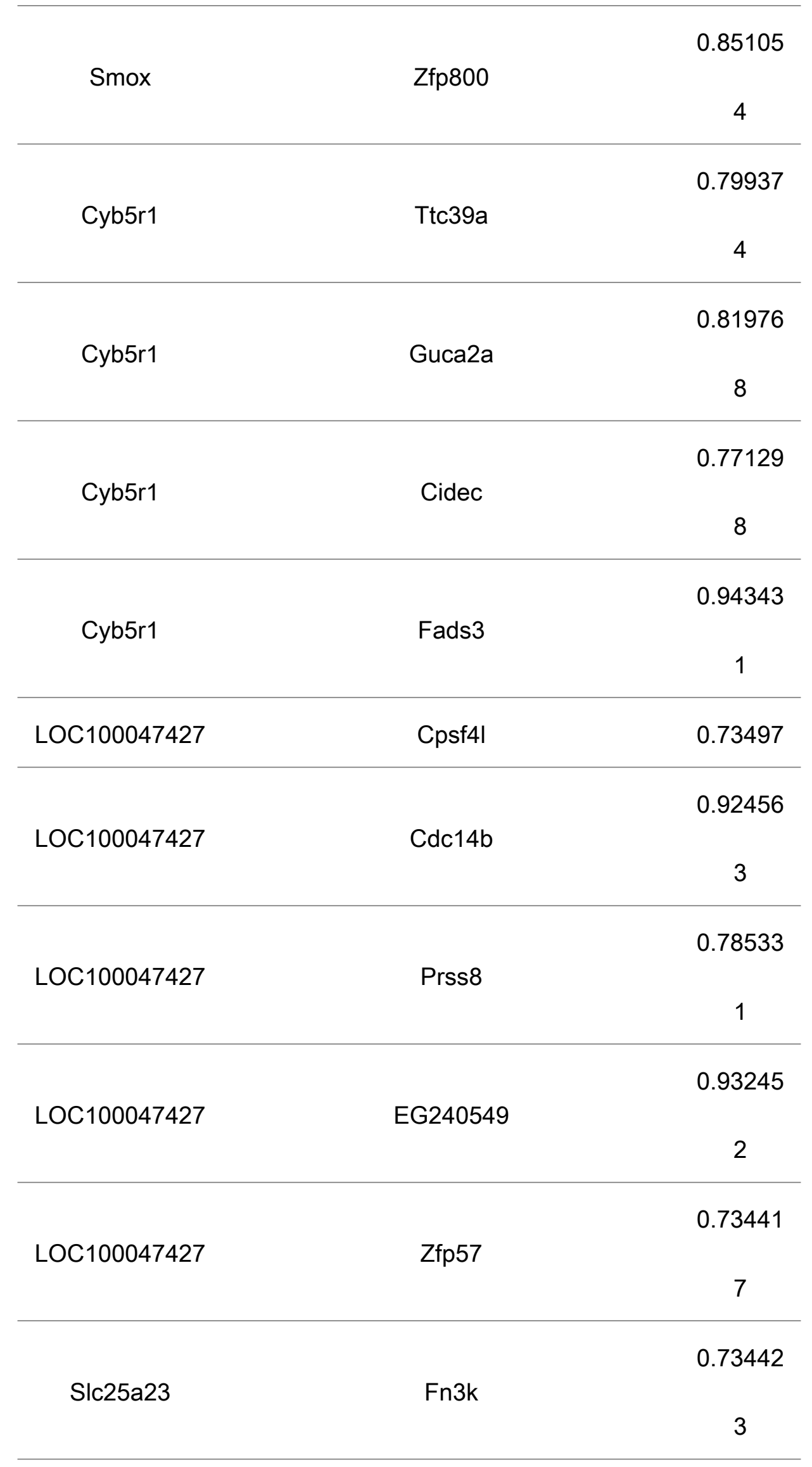




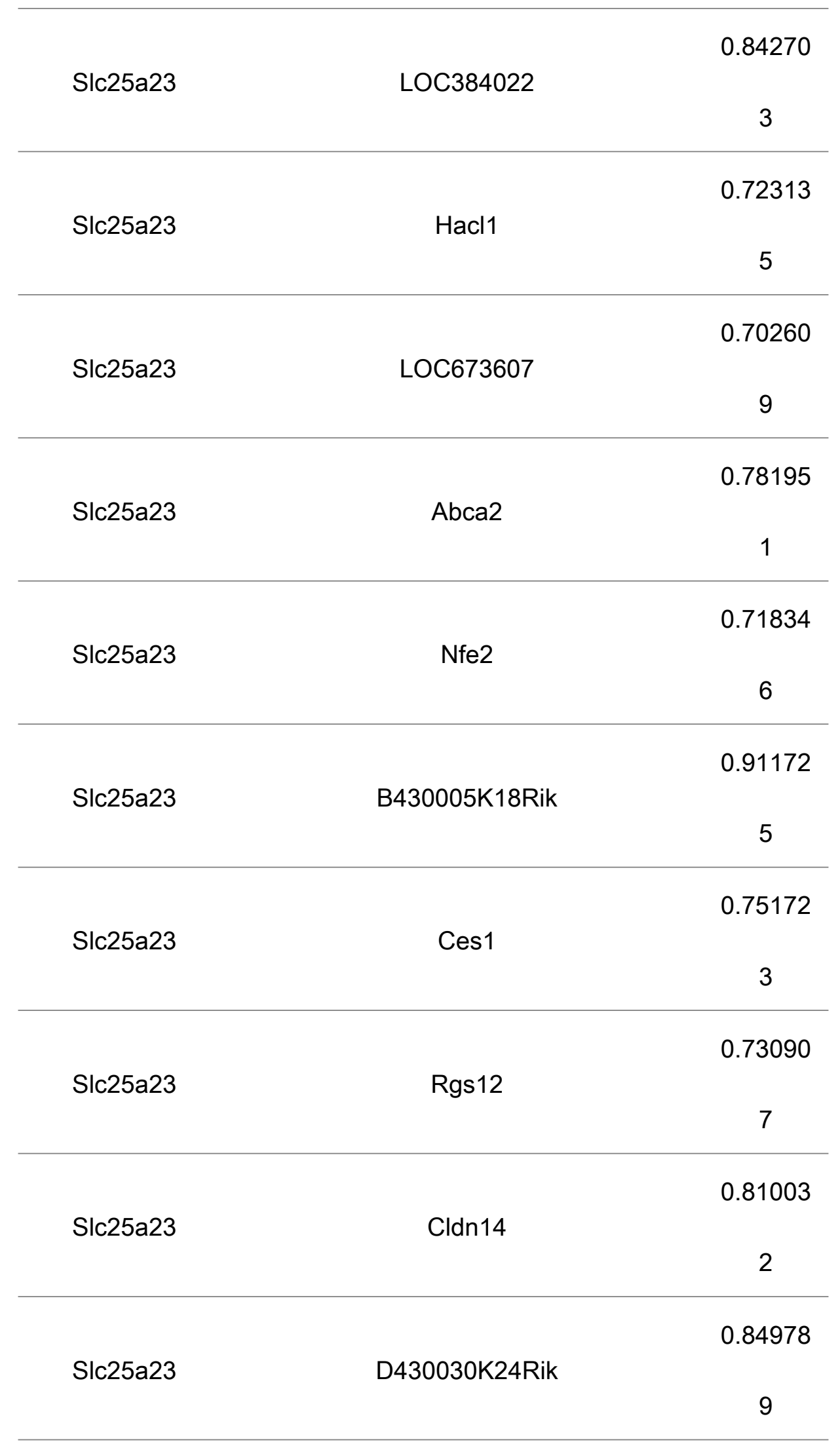




\begin{tabular}{|c|c|c|}
\hline Slc25a23 & Mov10 & $\begin{array}{c}0.77227 \\
5\end{array}$ \\
\hline LOC433886 & Fn3k & 0.71166 \\
\hline LOC433886 & Ngef & $\begin{array}{c}0.88403 \\
1\end{array}$ \\
\hline LOC433886 & Zfp750 & $\begin{array}{c}0.71999 \\
1\end{array}$ \\
\hline LOC433886 & Slc41a3 & $\begin{array}{c}0.93821 \\
4\end{array}$ \\
\hline LOC433886 & Ica1 & $\begin{array}{c}0.72701 \\
3\end{array}$ \\
\hline LOC433886 & Map3k6 & $\begin{array}{c}0.79363 \\
2\end{array}$ \\
\hline LOC433886 & 4432416J03Rik & $\begin{array}{c}0.77502 \\
8\end{array}$ \\
\hline LOC433886 & Slc27a3 & $\begin{array}{c}0.74078 \\
2\end{array}$ \\
\hline LOC433886 & C430002P19Rik & $\begin{array}{c}0.71336 \\
8\end{array}$ \\
\hline LOC433886 & Ces1 & $\begin{array}{c}0.75929 \\
1\end{array}$ \\
\hline
\end{tabular}




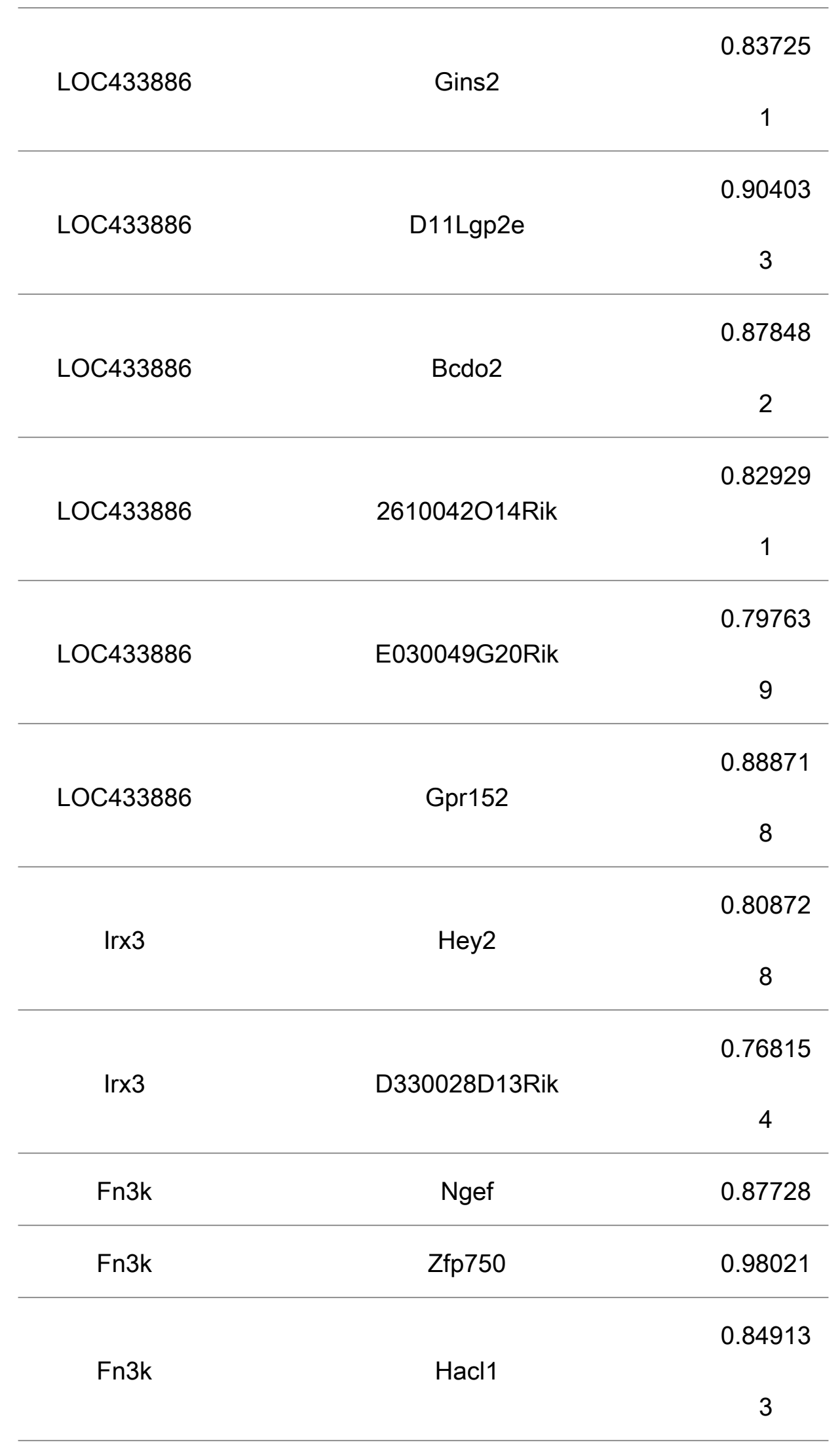




\begin{tabular}{|c|c|c|}
\hline \multirow[t]{2}{*}{ Fn3k } & Fam129a & $\begin{array}{c}0.96408 \\
2\end{array}$ \\
\hline & & 0.79266 \\
\hline \multirow[t]{3}{*}{ Fn3k } & Abca2 & \\
\hline & & 6 \\
\hline & & 0.92777 \\
\hline \multirow[t]{3}{*}{ Fn3k } & B430005K18Rik & \\
\hline & & 9 \\
\hline & & 0.97188 \\
\hline \multirow[t]{3}{*}{ Fn3k } & Ces1 & \\
\hline & & 1 \\
\hline & & 0.88025 \\
\hline \multirow[t]{3}{*}{ Fn3k } & Rgs12 & \\
\hline & & 8 \\
\hline & & 0.70088 \\
\hline \multirow[t]{3}{*}{ Fn3k } & LOC381738 & \\
\hline & & 7 \\
\hline & & 0.71812 \\
\hline \multirow[t]{3}{*}{ Fn3k } & E030049G20Rik & \\
\hline & & 6 \\
\hline & & 0.81880 \\
\hline \multirow[t]{3}{*}{ Fn3k } & Gpr152 & \\
\hline & & 7 \\
\hline & & 0.77826 \\
\hline \multirow[t]{3}{*}{ Olig1 } & LOC673607 & \\
\hline & & 5 \\
\hline & & 0.74064 \\
\hline \multirow[t]{2}{*}{ Olig1 } & Zc3h12d & \\
\hline & & 4 \\
\hline
\end{tabular}




\begin{tabular}{|c|c|c|}
\hline Olig1 & Gal3st1 & $\begin{array}{c}0.71377 \\
9\end{array}$ \\
\hline LOC384022 & B430005K18Rik & $\begin{array}{c}0.77957 \\
8\end{array}$ \\
\hline LOC384022 & LOC383215 & $\begin{array}{c}0.89432 \\
8\end{array}$ \\
\hline LOC384022 & LOC381738 & $\begin{array}{c}0.72804 \\
3\end{array}$ \\
\hline LOC384022 & Cldn14 & $\begin{array}{c}0.95440 \\
8\end{array}$ \\
\hline LOC384022 & D430030K24Rik & $\begin{array}{c}0.92259 \\
9\end{array}$ \\
\hline Ngef & Zfp750 & $\begin{array}{c}0.91736 \\
4\end{array}$ \\
\hline Ngef & Slc41a3 & $\begin{array}{c}0.91175 \\
9\end{array}$ \\
\hline Ngef & Sel1h & $\begin{array}{c}0.79980 \\
3\end{array}$ \\
\hline Ngef & Fam129a & $\begin{array}{c}0.89135 \\
7\end{array}$ \\
\hline
\end{tabular}




\begin{tabular}{|c|c|c|}
\hline \multirow[t]{2}{*}{ Ngef } & Ica1 & $\begin{array}{c}0.84547 \\
7\end{array}$ \\
\hline & & 0.74026 \\
\hline \multirow[t]{3}{*}{ Ngef } & 4432416J03Rik & \\
\hline & & 4 \\
\hline & & 0.82995 \\
\hline \multirow[t]{3}{*}{ Ngef } & Slc27a3 & \\
\hline & & 3 \\
\hline & & 0.73005 \\
\hline \multirow[t]{3}{*}{ Ngef } & B430005K18Rik & \\
\hline & & 6 \\
\hline & & 0.88772 \\
\hline \multirow[t]{3}{*}{ Ngef } & Ces1 & \\
\hline & & 9 \\
\hline & & 0.75045 \\
\hline \multirow[t]{3}{*}{ Ngef } & Gins2 & \\
\hline & & 4 \\
\hline & & 0.80818 \\
\hline \multirow[t]{3}{*}{ Ngef } & D11Lgp2e & \\
\hline & & 9 \\
\hline & & 0.74565 \\
\hline \multirow[t]{3}{*}{ Ngef } & D930009C14Rik & \\
\hline & & 3 \\
\hline & & 0.85833 \\
\hline \multirow[t]{3}{*}{ Ngef } & Bcdo2 & \\
\hline & & 3 \\
\hline & & 0.76665 \\
\hline \multirow[t]{2}{*}{ Ngef } & Rgs12 & \\
\hline & & 2 \\
\hline
\end{tabular}




\begin{tabular}{|c|c|c|}
\hline \multirow[t]{2}{*}{ Ngef } & 5730410E15Rik & $\begin{array}{c}0.77774 \\
4\end{array}$ \\
\hline & & 0.82704 \\
\hline \multirow[t]{3}{*}{ Ngef } & Gpr152 & \\
\hline & & 6 \\
\hline & & 0.74853 \\
\hline \multirow[t]{3}{*}{ Cdc42ep5 } & Hisppd2a & \\
\hline & & 1 \\
\hline & & 0.73430 \\
\hline \multirow[t]{3}{*}{ Cdc42ep5 } & Wnt5a & \\
\hline & & 7 \\
\hline & & 0.76973 \\
\hline \multirow[t]{3}{*}{ Сyp2b23 } & $\mathrm{H} 2-\mathrm{BI}$ & \\
\hline & & 1 \\
\hline & & 0.70905 \\
\hline \multirow[t]{3}{*}{ Cyp2b23 } & Slc7a11 & \\
\hline & & 8 \\
\hline & & 0.89573 \\
\hline \multirow[t]{3}{*}{ Cyp2b23 } & 2310014L17Rik & \\
\hline & & 7 \\
\hline & & 0.72593 \\
\hline \multirow[t]{3}{*}{ Zfp750 } & Slc41a3 & \\
\hline & & 5 \\
\hline & & 0.82157 \\
\hline \multirow[t]{3}{*}{ Zfp750 } & Hacl1 & \\
\hline & & 5 \\
\hline & & 0.99151 \\
\hline \multirow[t]{2}{*}{ Zfp 750} & Fam129a & \\
\hline & & 3 \\
\hline
\end{tabular}




\begin{tabular}{|c|c|c|}
\hline \multirow[t]{2}{*}{ Zfp750 } & Abca2 & $\begin{array}{c}0.74189 \\
4\end{array}$ \\
\hline & & 0.91598 \\
\hline \multirow[t]{3}{*}{ Zfp750 } & B430005K18Rik & \\
\hline & & 9 \\
\hline & & 0.96825 \\
\hline \multirow[t]{3}{*}{ Zfp750 } & Ces1 & \\
\hline & & 6 \\
\hline & & 0.74279 \\
\hline \multirow[t]{3}{*}{ Zfp750 } & Bcdo2 & \\
\hline & & 4 \\
\hline & & 0.86277 \\
\hline \multirow[t]{2}{*}{ Zfp750 } & Rgs12 & \\
\hline & & 8 \\
\hline \multirow[t]{2}{*}{ Zfp750 } & 5730410E15Rik & 0.75037 \\
\hline & & 0.72923 \\
\hline \multirow[t]{3}{*}{ Zfp750 } & LOC381738 & \\
\hline & & 4 \\
\hline & & 0.77035 \\
\hline \multirow[t]{2}{*}{ Zfp750 } & Gpr152 & \\
\hline & & 8 \\
\hline \multirow[t]{2}{*}{ Ppargc1b } & Chrna4 & 0.76661 \\
\hline & & 0.91170 \\
\hline \multirow[t]{3}{*}{ Ppargc1b } & Trim68 & \\
\hline & & 7 \\
\hline & & 0.75331 \\
\hline \multirow[t]{2}{*}{ II12rb1 } & 6720475J19Rik & \\
\hline & & 2 \\
\hline
\end{tabular}




\begin{tabular}{|c|c|c|}
\hline II12rb1 & Gt(ROSA)26asSor & $\begin{array}{c}0.78759 \\
3\end{array}$ \\
\hline \multirow[t]{2}{*}{ Bik } & Abca2 & 0.74999 \\
\hline & & 0.71335 \\
\hline \multirow[t]{2}{*}{ Bik } & B430005K18Rik & \\
\hline & & 7 \\
\hline \multirow[t]{2}{*}{ Bik } & Ces1 & 0.72601 \\
\hline & & 0.86479 \\
\hline \multirow[t]{3}{*}{ Bik } & Rgs12 & \\
\hline & & 2 \\
\hline & & 0.80248 \\
\hline \multirow[t]{3}{*}{ Bik } & Rock2 & \\
\hline & & 5 \\
\hline & & 0.85461 \\
\hline \multirow[t]{3}{*}{ Bik } & Mov10 & \\
\hline & & 2 \\
\hline & & 0.90983 \\
\hline \multirow[t]{3}{*}{ 1810020C02Rik } & Mcf2I & \\
\hline & & 6 \\
\hline & & 0.84256 \\
\hline \multirow[t]{2}{*}{ 1810020C02Rik } & 4833403D03Rik & \\
\hline & & 8 \\
\hline \multirow[t]{2}{*}{ 1810020C02Rik } & Ube2u & 0.70065 \\
\hline & & 0.85295 \\
\hline \multirow[t]{3}{*}{ 1810020C02Rik } & Bcdo2 & \\
\hline & & 9 \\
\hline & & 0.79531 \\
\hline \multirow[t]{2}{*}{ 1810020C02Rik } & Srd5a2 & \\
\hline & & 3 \\
\hline
\end{tabular}




\begin{tabular}{|c|c|c|}
\hline 1810020C02Rik & 5730410E15Rik & $\begin{array}{c}0.71059 \\
5\end{array}$ \\
\hline 1810020C02Rik & EG666009 & $\begin{array}{c}0.77330 \\
6\end{array}$ \\
\hline 1810020C02Rik & Zfp800 & $\begin{array}{c}0.87946 \\
5\end{array}$ \\
\hline Pdzk1ip1 & Ica1 & $\begin{array}{c}0.75463 \\
5\end{array}$ \\
\hline Pdzk1ip1 & 4432416J03Rik & $\begin{array}{c}0.95081 \\
1\end{array}$ \\
\hline Pdzk1ip1 & D930015E06Rik & $\begin{array}{c}0.80249 \\
8\end{array}$ \\
\hline Pdzk1ip1 & C430002P19Rik & $\begin{array}{c}0.79453 \\
4\end{array}$ \\
\hline Pdzk1ip1 & D11Lgp2e & $\begin{array}{c}0.81387 \\
1\end{array}$ \\
\hline Pdzk1ip1 & Ube2u & $\begin{array}{c}0.73825 \\
2\end{array}$ \\
\hline Pdzk1ip1 & Cnksr1 & $\begin{array}{c}0.73284 \\
7\end{array}$ \\
\hline
\end{tabular}




\begin{tabular}{|c|c|c|}
\hline \multirow[t]{2}{*}{ Pdzk1ip1 } & B230207L18Rik & $\begin{array}{c}0.83962 \\
1\end{array}$ \\
\hline & & 0.72790 \\
\hline \multirow[t]{3}{*}{ Pdzk1ip1 } & Pycr1 & \\
\hline & & 9 \\
\hline & & 0.71034 \\
\hline \multirow[t]{3}{*}{ Cpsf4l } & Cdc14b & \\
\hline & & 5 \\
\hline & & 0.89829 \\
\hline \multirow[t]{3}{*}{ Cpsf4l } & EG240549 & \\
\hline & & 8 \\
\hline & & 0.90577 \\
\hline \multirow[t]{2}{*}{ Cpsf4l } & 2610528J11Rik & \\
\hline & & 6 \\
\hline \multirow[t]{2}{*}{ Slc41a3 } & Sel1h & 0.80029 \\
\hline & & 0.83060 \\
\hline \multirow[t]{3}{*}{ Slc41a3 } & Ica1 & \\
\hline & & 2 \\
\hline & & 0.80121 \\
\hline \multirow[t]{3}{*}{ Slc41a3 } & Map3k6 & \\
\hline & & 7 \\
\hline & & 0.81563 \\
\hline \multirow[t]{3}{*}{ Slc41a3 } & 4432416J03Rik & \\
\hline & & 9 \\
\hline & & 0.72969 \\
\hline \multirow[t]{3}{*}{ Slc41a3 } & Slc27a3 & \\
\hline & & 1 \\
\hline & & 0.80117 \\
\hline \multirow[t]{2}{*}{ Slc41a3 } & C430002P19Rik & \\
\hline & & 5 \\
\hline
\end{tabular}




\begin{tabular}{|c|c|c|}
\hline \multirow[t]{2}{*}{ Slc41a3 } & Ces1 & $\begin{array}{c}0.74557 \\
4\end{array}$ \\
\hline & & 0.82489 \\
\hline \multirow[t]{3}{*}{ Slc41a3 } & Gins2 & \\
\hline & & 5 \\
\hline & & 0.85532 \\
\hline \multirow[t]{3}{*}{ Slc41a3 } & D11Lgp2e & \\
\hline & & 1 \\
\hline & & 0.80700 \\
\hline \multirow[t]{3}{*}{ Slc41a3 } & Bcdo2 & \\
\hline & & 6 \\
\hline & & 0.73115 \\
\hline \multirow[t]{3}{*}{ Slc41a3 } & 2610042O14Rik & \\
\hline & & 4 \\
\hline & & 0.76540 \\
\hline \multirow[t]{3}{*}{ Slc41a3 } & B230207L18Rik & \\
\hline & & 2 \\
\hline & & 0.82577 \\
\hline \multirow[t]{3}{*}{ Slc41a3 } & Gpr152 & \\
\hline & & 2 \\
\hline & & 0.79259 \\
\hline \multirow[t]{3}{*}{ Cdc14b } & Prss8 & \\
\hline & & 8 \\
\hline & & 0.91901 \\
\hline \multirow[t]{3}{*}{ Cdc14b } & EG240549 & \\
\hline & & 1 \\
\hline & & 0.74379 \\
\hline \multirow[t]{2}{*}{ Cdc14b } & 2610528J11Rik & \\
\hline & & 9 \\
\hline
\end{tabular}




\begin{tabular}{|c|c|c|}
\hline Tjp3 & Hacl1 & $\begin{array}{c}0.71893 \\
6\end{array}$ \\
\hline \multirow{3}{*}{ Tjp3 } & \multirow{3}{*}{ Slc44a3 } & 0.92210 \\
\hline & & \\
\hline & & 8 \\
\hline \multirow[t]{2}{*}{ Tjp3 } & Zc3h12d & 0.90573 \\
\hline & & 0.71692 \\
\hline \multirow[t]{3}{*}{ Tjp3 } & Grb14 & \\
\hline & & 6 \\
\hline & & 0.79352 \\
\hline \multirow[t]{3}{*}{ Prss8 } & Slc27a3 & \\
\hline & & 7 \\
\hline & & 0.82452 \\
\hline \multirow[t]{3}{*}{ Prss8 } & Zfp57 & \\
\hline & & 5 \\
\hline & & 0.79852 \\
\hline \multirow[t]{3}{*}{ Prss8 } & 1810046K07Rik & \\
\hline & & 1 \\
\hline & & 0.78205 \\
\hline \multirow[t]{3}{*}{ Prss8 } & Mpdz & \\
\hline & & 9 \\
\hline & & 0.71319 \\
\hline \multirow[t]{3}{*}{ Mcc } & Impa2 & \\
\hline & & 3 \\
\hline & & 0.82703 \\
\hline \multirow[t]{3}{*}{ Rab17 } & Slc13a2 & \\
\hline & & 3 \\
\hline & & 0.70532 \\
\hline \multirow[t]{2}{*}{ A530020H22Rik } & Cnksr1 & \\
\hline & & 8 \\
\hline
\end{tabular}




\begin{tabular}{|c|c|c|}
\hline \multirow[t]{2}{*}{ A530020H22Rik } & Oip5 & $\begin{array}{c}0.85716 \\
1\end{array}$ \\
\hline & & 0.88281 \\
\hline \multirow[t]{2}{*}{ Atg9b } & Far1 & \\
\hline & & 6 \\
\hline \multirow[t]{2}{*}{ Atp8b1 } & Lrtm1 & 0.8523 \\
\hline & & 0.81463 \\
\hline \multirow[t]{3}{*}{ Atp8b1 } & 9130409I23Rik & \\
\hline & & 3 \\
\hline & & 0.82426 \\
\hline \multirow[t]{2}{*}{ Pcbp3 } & 4930581F22Rik & \\
\hline & & 5 \\
\hline Pcbp3 & C730014E05Rik & 0.78378 \\
\hline \multirow[t]{2}{*}{ Pcbp3 } & A730014O07Rik & 0.95174 \\
\hline & & 0.70850 \\
\hline \multirow[t]{3}{*}{ 1600032L17Rik } & Slc13a2 & \\
\hline & & 6 \\
\hline & & 0.78639 \\
\hline \multirow[t]{3}{*}{ A630034I12Rik } & Niban & \\
\hline & & 7 \\
\hline & & 0.72498 \\
\hline \multirow[t]{3}{*}{ A630034I12Rik } & D330028D13Rik & \\
\hline & & 2 \\
\hline & & 0.73577 \\
\hline \multirow[t]{3}{*}{ A630034I12Rik } & 5830411E10Rik & \\
\hline & & 1 \\
\hline & & 0.72495 \\
\hline \multirow[t]{2}{*}{ Srebf1 } & Ncald & \\
\hline & & 4 \\
\hline
\end{tabular}




\begin{tabular}{|c|c|c|}
\hline \multirow[t]{2}{*}{ Hacl1 } & Fam129a & $\begin{array}{c}0.83819 \\
3\end{array}$ \\
\hline & & 0.77163 \\
\hline \multirow[t]{3}{*}{ Hacl1 } & Slc44a3 & \\
\hline & & 6 \\
\hline & & 0.84196 \\
\hline \multirow[t]{3}{*}{ Hacl1 } & B430005K18Rik & \\
\hline & & 2 \\
\hline & & 0.82332 \\
\hline \multirow[t]{3}{*}{ Hacl1 } & Ces1 & \\
\hline & & 6 \\
\hline & & 0.89259 \\
\hline \multirow[t]{3}{*}{ Hacl1 } & Grb14 & \\
\hline & & 5 \\
\hline & & 0.82349 \\
\hline \multirow[t]{3}{*}{ Hacl1 } & Acpp & \\
\hline & & 1 \\
\hline & & 0.86445 \\
\hline \multirow[t]{3}{*}{ Hacl1 } & LOC381738 & \\
\hline & & 6 \\
\hline & & 0.74611 \\
\hline \multirow[t]{3}{*}{ Hacl1 } & E030049G20Rik & \\
\hline & & 9 \\
\hline & & 0.71899 \\
\hline \multirow[t]{3}{*}{ Hacl1 } & Zfp800 & \\
\hline & & 5 \\
\hline & & 0.72980 \\
\hline \multirow[t]{2}{*}{ Ttc39a } & Mcf2I & \\
\hline & & 6 \\
\hline Ttc39a & Guca2a & 0.84398 \\
\hline
\end{tabular}




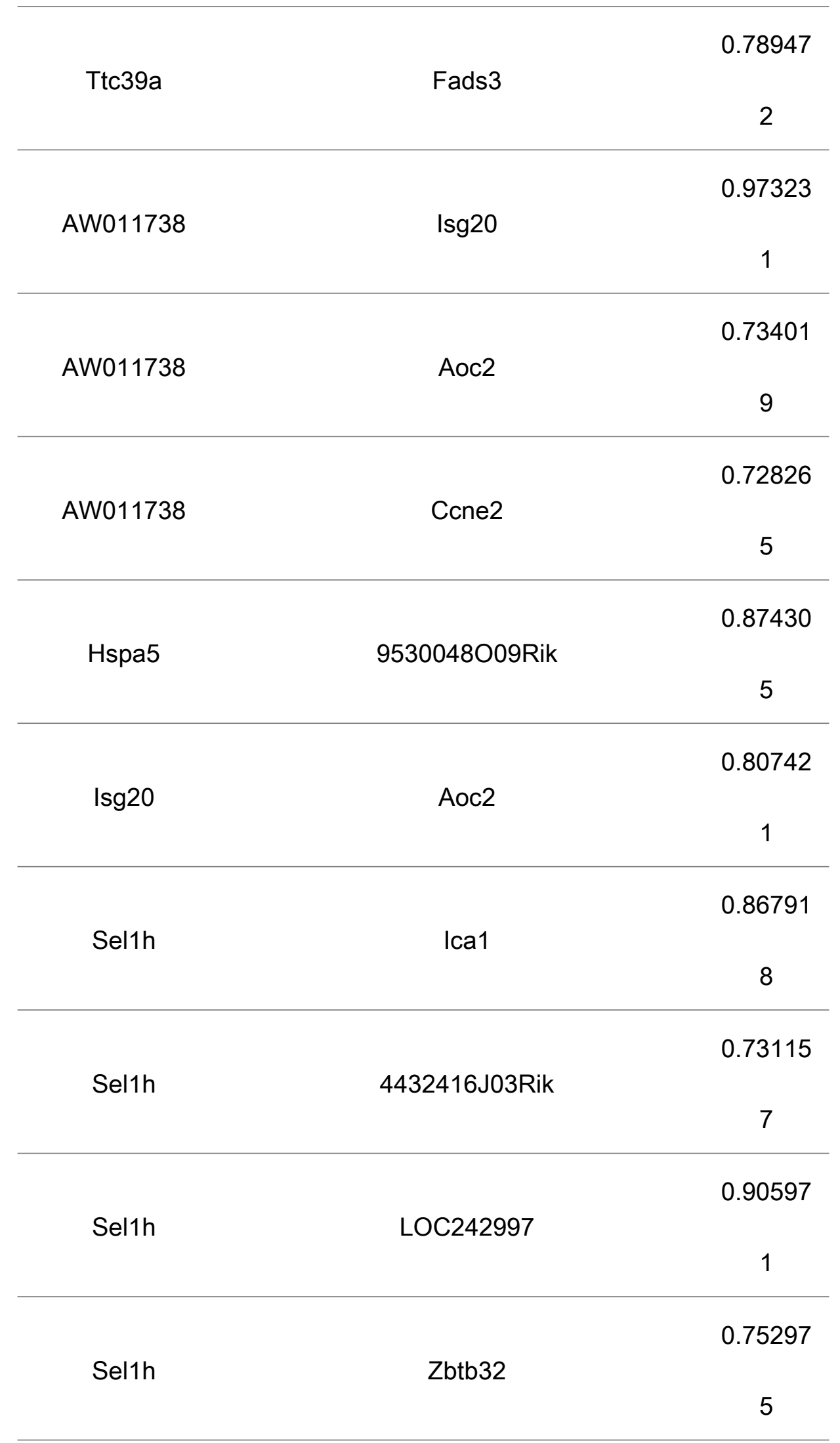




\begin{tabular}{|c|c|c|}
\hline \multirow[t]{2}{*}{ Sel1h } & C430002P19Rik & $\begin{array}{c}0.70972 \\
2\end{array}$ \\
\hline & & 0.73357 \\
\hline \multirow[t]{2}{*}{ Sel1h } & 1810015A11Rik & \\
\hline & & 1 \\
\hline \multirow[t]{2}{*}{ Sel1h } & D930009C14Rik & 0.7508 \\
\hline & & 0.71130 \\
\hline \multirow[t]{3}{*}{ Sel1h } & Pycr1 & \\
\hline & & 9 \\
\hline & & 0.73132 \\
\hline \multirow[t]{3}{*}{ Niban } & 1810015A11Rik & \\
\hline & & 4 \\
\hline & & 0.77492 \\
\hline \multirow[t]{3}{*}{ Niban } & Bves & \\
\hline & & 6 \\
\hline & & 0.79324 \\
\hline \multirow[t]{3}{*}{ Niban } & D930009C14Rik & \\
\hline & & 9 \\
\hline & & 0.75989 \\
\hline \multirow[t]{3}{*}{ Niban } & 5730410E15Rik & \\
\hline & & 5 \\
\hline & & 0.87814 \\
\hline \multirow[t]{2}{*}{ Niban } & 1810008K04Rik & \\
\hline & & 4 \\
\hline \multirow[t]{2}{*}{ Niban } & Nphp3 & 0.80205 \\
\hline & & 0.98081 \\
\hline \multirow[t]{2}{*}{ Niban } & 5830411E10Rik & \\
\hline & & 4 \\
\hline
\end{tabular}




\begin{tabular}{|c|c|c|}
\hline \multirow[t]{2}{*}{ Niban } & Ranbp3I & $\begin{array}{c}0.88702 \\
7\end{array}$ \\
\hline & & 0.75850 \\
\hline \multirow[t]{3}{*}{ Hisppd2a } & DXBwg1396e & \\
\hline & & 4 \\
\hline & & 0.86183 \\
\hline \multirow[t]{3}{*}{ Hisppd2a } & Slc30a3 & \\
\hline & & 6 \\
\hline & & 0.99555 \\
\hline \multirow[t]{3}{*}{ Csn3 } & Atf3 & \\
\hline & & 2 \\
\hline & & 0.72490 \\
\hline \multirow[t]{3}{*}{ Csn3 } & LOC212399 & \\
\hline & & 6 \\
\hline & & 0.82046 \\
\hline \multirow[t]{2}{*}{ Csn3 } & Csnk & \\
\hline & & 8 \\
\hline \multirow[t]{2}{*}{ Csn3 } & Alkbh & 0.88287 \\
\hline & & 0.71888 \\
\hline \multirow[t]{3}{*}{ Fam129a } & Abca2 & \\
\hline & & 2 \\
\hline & & 0.89724 \\
\hline \multirow[t]{3}{*}{ Fam129a } & B430005K18Rik & \\
\hline & & 4 \\
\hline & & 0.93403 \\
\hline \multirow[t]{3}{*}{ Fam129a } & Ces1 & \\
\hline & & 7 \\
\hline & & 0.70686 \\
\hline \multirow[t]{2}{*}{ Fam129a } & Bves & \\
\hline & & 8 \\
\hline
\end{tabular}




\begin{tabular}{|c|c|c|}
\hline Fam129a & Grb14 & 0.75399 \\
\hline & & 0.73065 \\
\hline \multirow[t]{3}{*}{ Fam129a } & Bcdo2 & \\
\hline & & 4 \\
\hline & & 0.82090 \\
\hline \multirow[t]{3}{*}{ Fam129a } & Rgs12 & \\
\hline & & 3 \\
\hline & & 0.78671 \\
\hline \multirow[t]{3}{*}{ Fam129a } & 5730410E15Rik & \\
\hline & & 7 \\
\hline & & 0.73444 \\
\hline \multirow[t]{3}{*}{ Fam129a } & 1810008K04Rik & \\
\hline & & 8 \\
\hline & & 0.70570 \\
\hline \multirow[t]{3}{*}{ Fam129a } & Nphp3 & \\
\hline & & 1 \\
\hline & & 0.79069 \\
\hline \multirow[t]{2}{*}{ Fam129a } & LOC381738 & \\
\hline & & 7 \\
\hline \multirow[t]{2}{*}{ Fam129a } & Ranbp3l & 0.75333 \\
\hline & & 0.70637 \\
\hline \multirow[t]{3}{*}{ Fam129a } & Gpr152 & \\
\hline & & 4 \\
\hline & & 0.83993 \\
\hline \multirow[t]{3}{*}{ EG240549 } & 2610528J11Rik & \\
\hline & & 6 \\
\hline & & 0.72109 \\
\hline \multirow[t]{2}{*}{ Far1 } & 5830411E10Rik & \\
\hline & & 3 \\
\hline
\end{tabular}




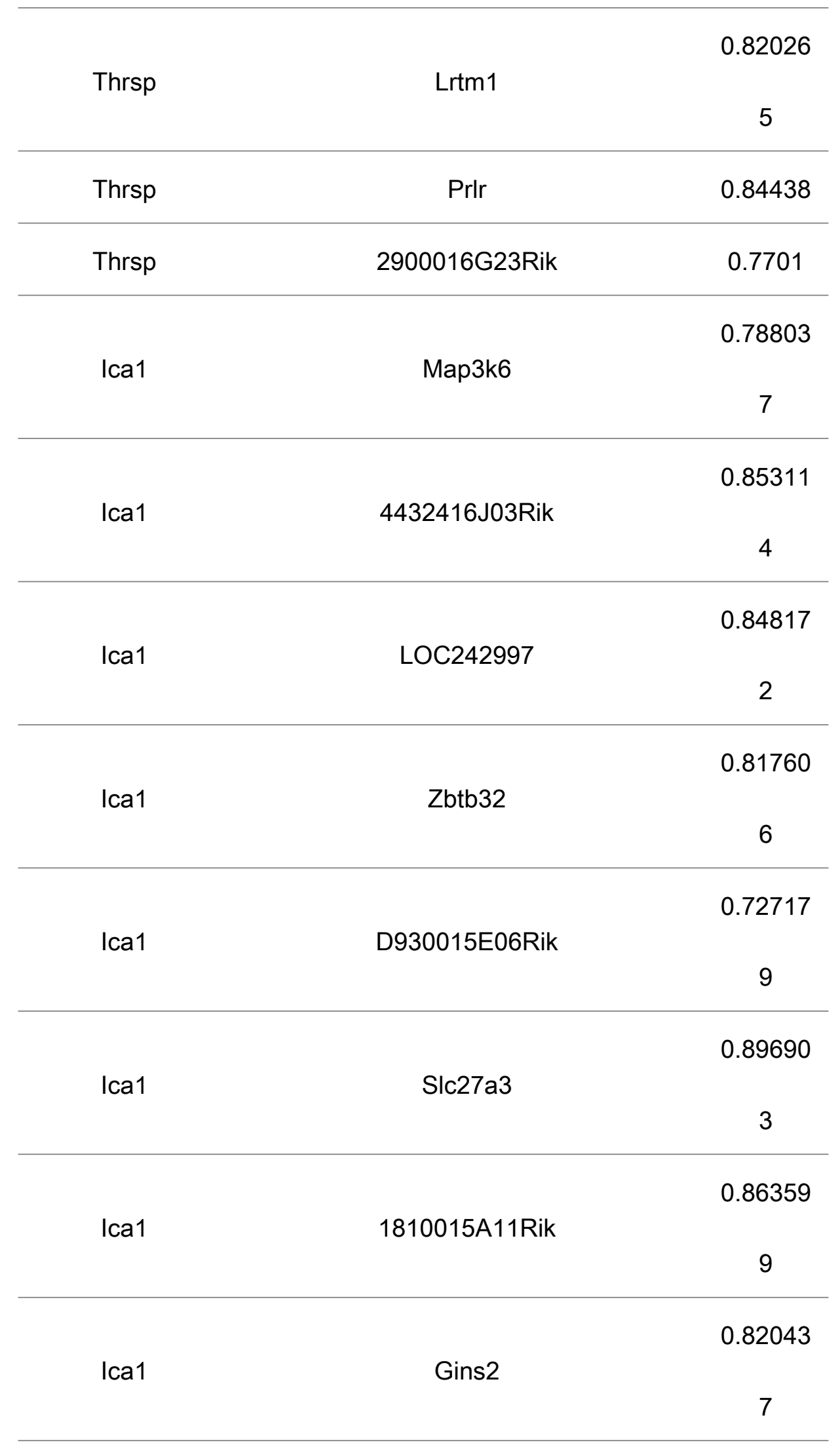




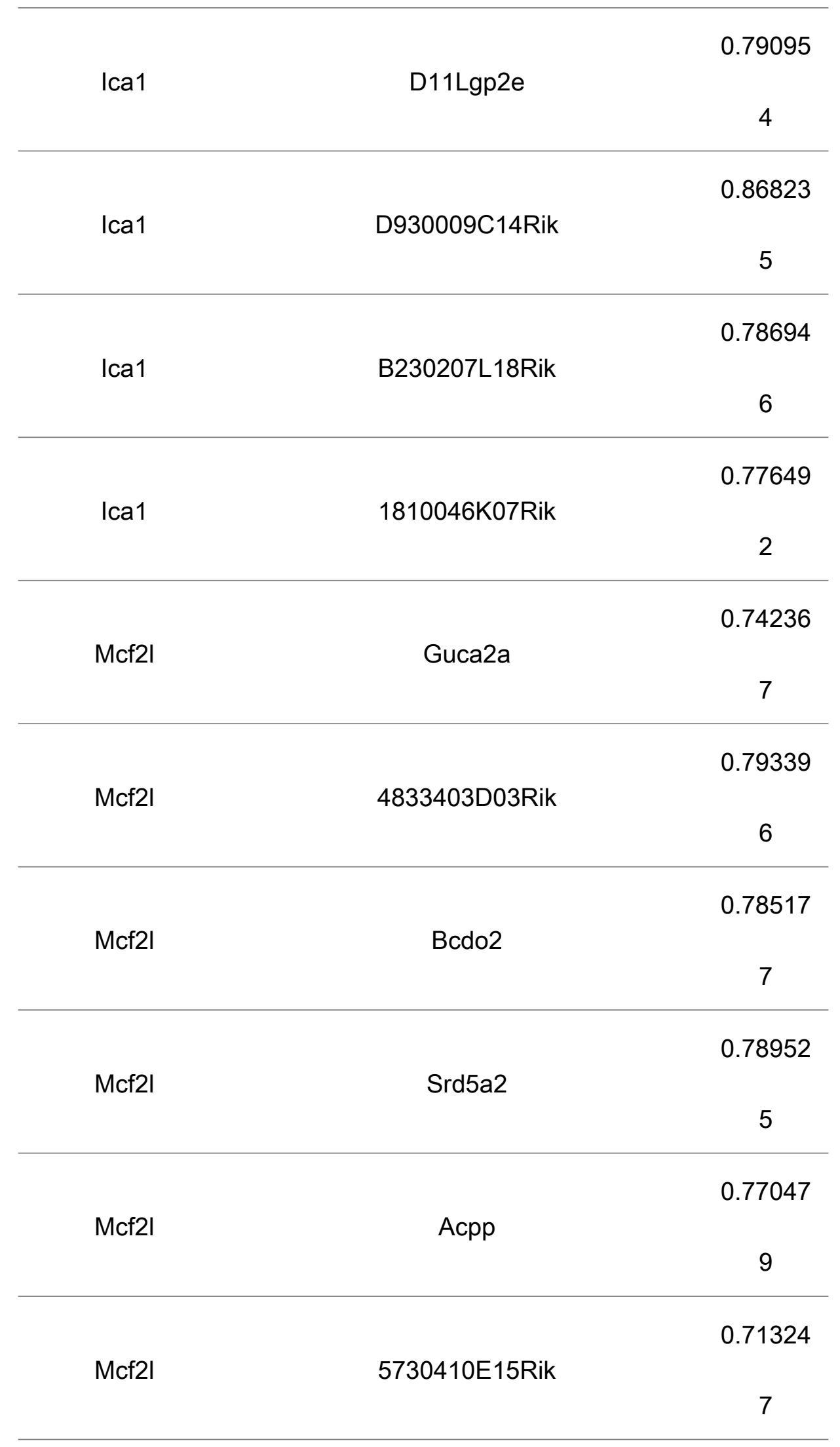




\begin{tabular}{|c|c|c|}
\hline Mcf2I & Zfp800 & $\begin{array}{c}0.95315 \\
1\end{array}$ \\
\hline Cabc1 & Zfp57 & $\begin{array}{c}0.90227 \\
9\end{array}$ \\
\hline Cabc1 & Acpp & $\begin{array}{c}0.76732 \\
5\end{array}$ \\
\hline Cabc1 & E030049G20Rik & $\begin{array}{c}0.73117 \\
7\end{array}$ \\
\hline Cabc1 & Mpdz & 0.90485 \\
\hline Map3k6 & 4432416J03Rik & $\begin{array}{c}0.77795 \\
1\end{array}$ \\
\hline Map3k6 & D930015E06Rik & $\begin{array}{c}0.71603 \\
2\end{array}$ \\
\hline Map3k6 & Slc27a3 & $\begin{array}{c}0.76392 \\
5\end{array}$ \\
\hline Map3k6 & Gins2 & $\begin{array}{c}0.97975 \\
9\end{array}$ \\
\hline Map3k6 & D11Lgp2e & $\begin{array}{c}0.80351 \\
9\end{array}$ \\
\hline Map3k6 & 2610042014Rik & $\begin{array}{c}0.90964 \\
3\end{array}$ \\
\hline
\end{tabular}




\begin{tabular}{|c|c|c|}
\hline Map3k6 & B230207L18Rik & $\begin{array}{c}0.87782 \\
7\end{array}$ \\
\hline Map3k6 & 1810046K07Rik & $\begin{array}{c}0.83406 \\
3\end{array}$ \\
\hline Ifi47 & Tgm1 & $\begin{array}{c}0.71396 \\
3\end{array}$ \\
\hline 1500017E21Rik & C330001K17Rik & $\begin{array}{c}0.71423 \\
3\end{array}$ \\
\hline 4432416J03Rik & D930015E06Rik & $\begin{array}{c}0.77723 \\
3\end{array}$ \\
\hline 4432416J03Rik & Slc27a3 & $\begin{array}{c}0.77067 \\
4\end{array}$ \\
\hline 4432416J03Rik & C430002P19Rik & $\begin{array}{c}0.83459 \\
5\end{array}$ \\
\hline 4432416J03Rik & Gins2 & $\begin{array}{c}0.76285 \\
5\end{array}$ \\
\hline 4432416J03Rik & D11Lgp2e & 0.93932 \\
\hline 4432416J03Rik & Ube2u & $\begin{array}{c}0.72545 \\
6\end{array}$ \\
\hline 4432416J03Rik & Bcdo2 & $\begin{array}{c}0.78155 \\
1\end{array}$ \\
\hline
\end{tabular}




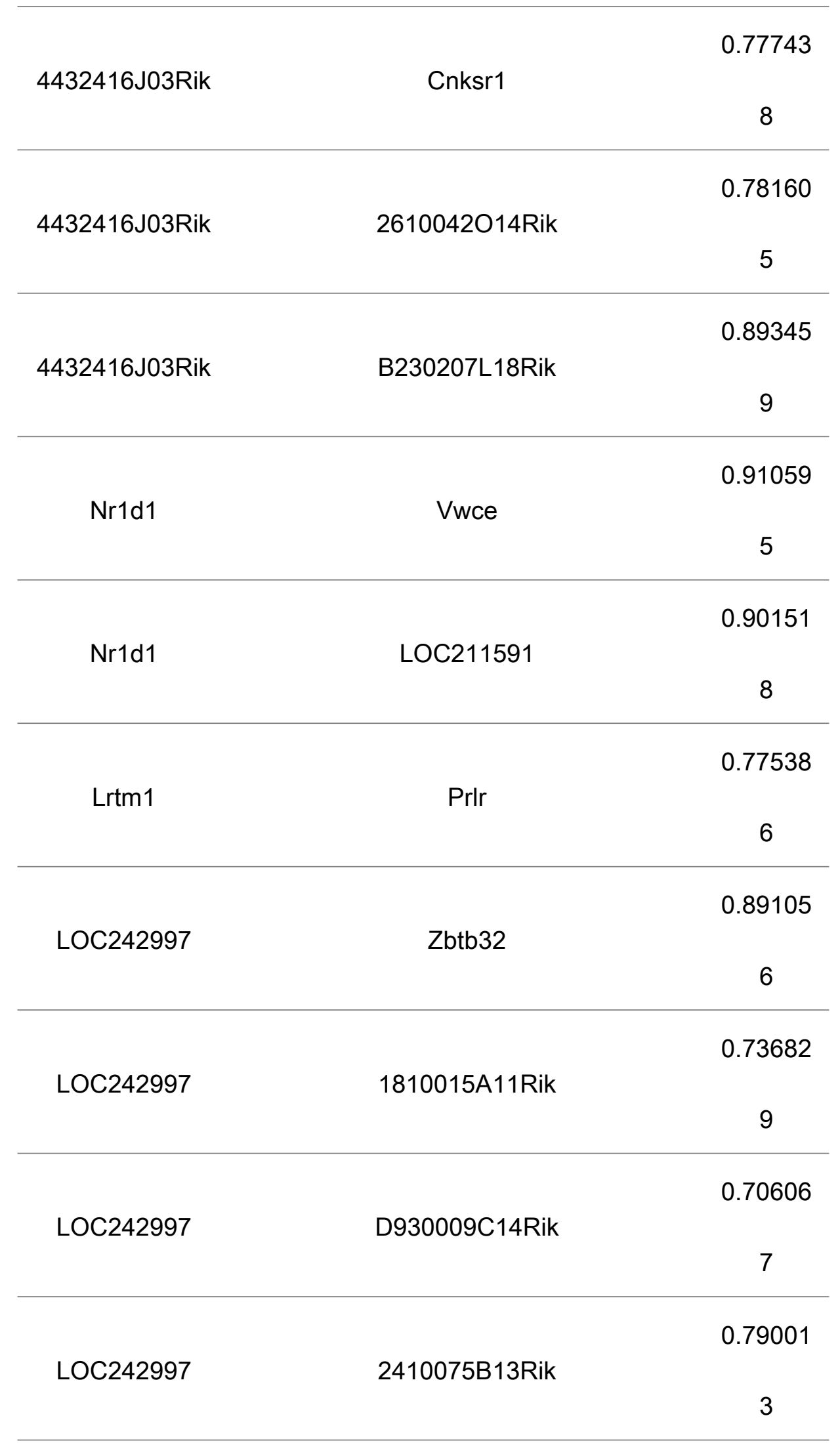




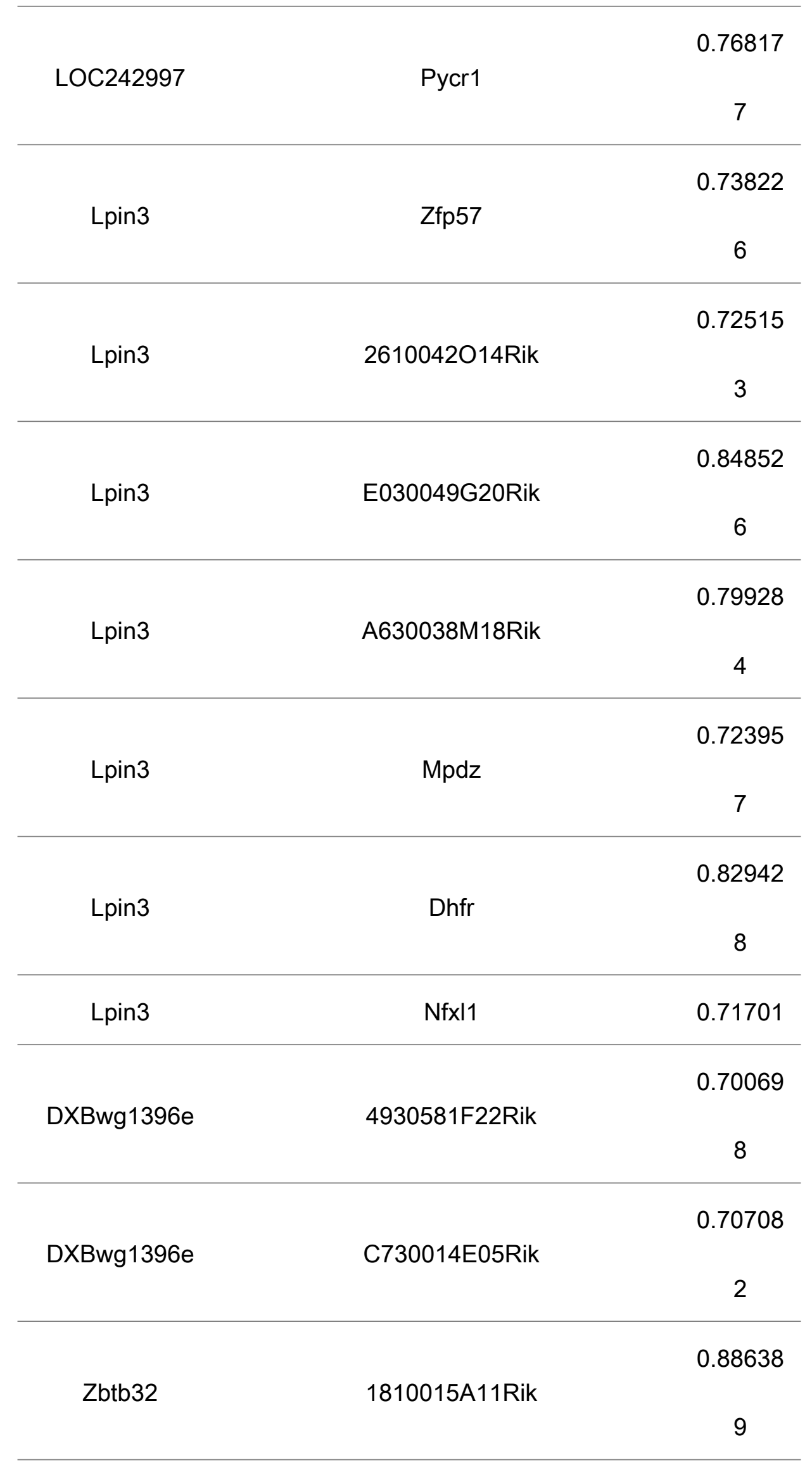




\begin{tabular}{|c|c|c|}
\hline Zbtb32 & D930009C14Rik & $\begin{array}{c}0.80591 \\
6\end{array}$ \\
\hline & & 0.73659 \\
\hline \multirow[t]{3}{*}{ Zbtb32 } & 2410075B13Rik & \\
\hline & & 9 \\
\hline & & 0.70156 \\
\hline \multirow[t]{3}{*}{ Zbtb32 } & Pycr1 & \\
\hline & & 6 \\
\hline & & 0.92166 \\
\hline \multirow[t]{3}{*}{ Vwce } & LOC211591 & \\
\hline & & 5 \\
\hline & & 0.70250 \\
\hline \multirow[t]{3}{*}{ Vwce } & Mpdz & \\
\hline & & 1 \\
\hline & & 0.78028 \\
\hline \multirow[t]{2}{*}{ Prlr } & 1700012H05Rik & \\
\hline & & 4 \\
\hline \multirow[t]{2}{*}{ D930015E06Rik } & 2410075B13Rik & 0.75405 \\
\hline & & 0.83965 \\
\hline \multirow[t]{3}{*}{ D930015E06Rik } & B230207L18Rik & \\
\hline & & 6 \\
\hline & & 0.73862 \\
\hline \multirow[t]{3}{*}{ D930015E06Rik } & 1810046K07Rik & \\
\hline & & 3 \\
\hline & & 0.78659 \\
\hline \multirow[t]{3}{*}{ E030040G24Rik } & LOC212399 & \\
\hline & & 4 \\
\hline & & 0.83587 \\
\hline \multirow[t]{2}{*}{ E030040G24Rik } & LOC100041004 & \\
\hline & & 6 \\
\hline
\end{tabular}




\begin{tabular}{|c|c|c|}
\hline 9130409I23Rik & C430002P19Rik & $\begin{array}{c}0.75904 \\
4\end{array}$ \\
\hline 9130409I23Rik & Ube2u & $\begin{array}{c}0.70626 \\
8\end{array}$ \\
\hline 9130409I23Rik & Cnksr1 & $\begin{array}{c}0.71674 \\
4\end{array}$ \\
\hline 9130409I23Rik & 5730593N15Rik & $\begin{array}{c}0.79277 \\
1\end{array}$ \\
\hline LOC219106 & 4833403D03Rik & $\begin{array}{c}0.81797 \\
6\end{array}$ \\
\hline LOC219106 & St6galnac4 & $\begin{array}{c}0.87152 \\
3\end{array}$ \\
\hline $\begin{array}{c}\text { scl0001883.1_1 } \\
12\end{array}$ & Prr16 & $\begin{array}{c}0.84763 \\
7\end{array}$ \\
\hline Chrna4 & Pnpla5 & $\begin{array}{c}0.77946 \\
7\end{array}$ \\
\hline Chrna4 & Trim68 & $\begin{array}{c}0.84188 \\
1\end{array}$ \\
\hline Slc44a3 & Zc3h12d & $\begin{array}{c}0.81714 \\
3\end{array}$ \\
\hline
\end{tabular}




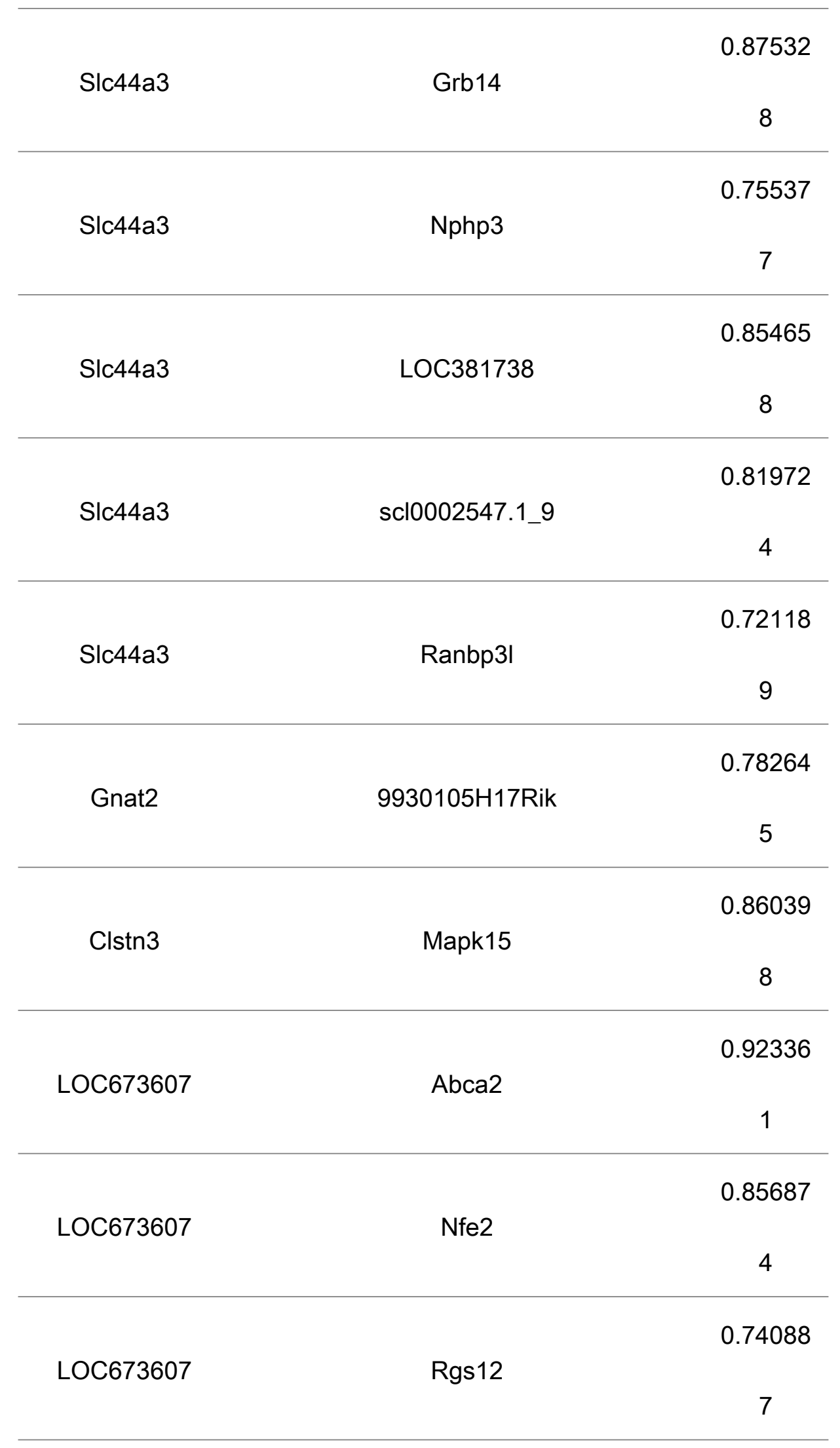




\begin{tabular}{|c|c|c|}
\hline \multirow[t]{2}{*}{ Slc27a3 } & 1810015A11Rik & $\begin{array}{c}0.79757 \\
6\end{array}$ \\
\hline & & 0.84160 \\
\hline \multirow[t]{3}{*}{ Slc27a3 } & Gins2 & \\
\hline & & 2 \\
\hline & & 0.80798 \\
\hline \multirow[t]{3}{*}{ Slc27a3 } & D11Lgp2e & \\
\hline & & 6 \\
\hline & & 0.77600 \\
\hline \multirow[t]{3}{*}{ Slc27a3 } & Zfp57 & \\
\hline & & 4 \\
\hline & & 0.85146 \\
\hline \multirow[t]{2}{*}{ Slc27a3 } & D930009C14Rik & \\
\hline & & 6 \\
\hline \multirow[t]{2}{*}{ Slc27a3 } & Bcdo2 & 0.70481 \\
\hline & & 0.74225 \\
\hline \multirow[t]{3}{*}{ Slc27a3 } & 2610042O14Rik & \\
\hline & & 2 \\
\hline & & 0.86453 \\
\hline \multirow[t]{3}{*}{ Slc27a3 } & 1810046K07Rik & \\
\hline & & 6 \\
\hline & & 0.74974 \\
\hline \multirow[t]{3}{*}{ Abca2 } & Nfe2 & \\
\hline & & 9 \\
\hline & & 0.82091 \\
\hline \multirow[t]{3}{*}{ Abca2 } & B430005K18Rik & \\
\hline & & 4 \\
\hline & & 0.73791 \\
\hline \multirow[t]{2}{*}{ Abca2 } & Ces1 & \\
\hline & & 2 \\
\hline
\end{tabular}




\begin{tabular}{|c|c|c|}
\hline Abca2 & Rgs12 & $\begin{array}{c}0.92432 \\
4\end{array}$ \\
\hline Abca2 & D430030K24Rik & 0.76032 \\
\hline Abca2 & Mov10 & $\begin{array}{c}0.74052 \\
1\end{array}$ \\
\hline Guca2a & 4833403D03Rik & $\begin{array}{c}0.74635 \\
5\end{array}$ \\
\hline $\mathrm{Nfe} 2$ & D430030K24Rik & $\begin{array}{c}0.70869 \\
8\end{array}$ \\
\hline $\mathrm{Nfe} 2$ & 9330129D05Rik & $\begin{array}{c}0.72182 \\
3\end{array}$ \\
\hline Atf3 & LOC212399 & $\begin{array}{c}0.71372 \\
4\end{array}$ \\
\hline Atf3 & Csnk & $\begin{array}{c}0.83542 \\
3\end{array}$ \\
\hline Atf3 & Alkbh & 0.90253 \\
\hline B430005K18Rik & Gas7 & $\begin{array}{c}0.70515 \\
5\end{array}$ \\
\hline B430005K18Rik & Ces1 & $\begin{array}{c}0.94089 \\
3\end{array}$ \\
\hline
\end{tabular}




\begin{tabular}{|c|c|c|}
\hline B430005K18Rik & Rgs12 & $\begin{array}{c}0.86688 \\
2\end{array}$ \\
\hline B430005K18Rik & LOC381738 & $\begin{array}{c}0.70570 \\
5\end{array}$ \\
\hline B430005K18Rik & Cldn14 & $\begin{array}{c}0.73917 \\
2\end{array}$ \\
\hline B430005K18Rik & D430030K24Rik & $\begin{array}{c}0.79226 \\
8\end{array}$ \\
\hline B430005K18Rik & Mov10 & $\begin{array}{c}0.75962 \\
6\end{array}$ \\
\hline $\mathrm{H} 2-\mathrm{BI}$ & 2310014L17Rik & 0.75497 \\
\hline B430114K07Rik & 1600016N20Rik & $\begin{array}{c}0.88904 \\
4\end{array}$ \\
\hline B430114K07Rik & LOC243368 & $\begin{array}{c}0.93164 \\
3\end{array}$ \\
\hline Gas7 & Ces1 & $\begin{array}{c}0.70317 \\
7\end{array}$ \\
\hline C430002P19Rik & D11Lgp2e & $\begin{array}{c}0.77586 \\
7\end{array}$ \\
\hline C430002P19Rik & Ube2u & $\begin{array}{c}0.84879 \\
4\end{array}$ \\
\hline
\end{tabular}




\begin{tabular}{|c|c|c|}
\hline C430002P19Rik & Bcdo2 & $\begin{array}{c}0.72540 \\
2\end{array}$ \\
\hline C430002P19Rik & Cnksr1 & $\begin{array}{c}0.83359 \\
9\end{array}$ \\
\hline C430002P19Rik & B230207L18Rik & $\begin{array}{c}0.75450 \\
8\end{array}$ \\
\hline 1810015A11Rik & D930009C14Rik & $\begin{array}{c}0.93981 \\
2\end{array}$ \\
\hline 1810015A11Rik & 5830411E10Rik & $\begin{array}{c}0.71017 \\
6\end{array}$ \\
\hline LOC100043986 & St6galnac4 & $\begin{array}{c}0.83710 \\
9\end{array}$ \\
\hline LOC100043986 & Btg3 & $\begin{array}{c}0.70096 \\
7\end{array}$ \\
\hline Rnd2 & Zc3h12d & $\begin{array}{c}0.70977 \\
4\end{array}$ \\
\hline Rnd2 & Grb14 & $\begin{array}{c}0.73816 \\
3\end{array}$ \\
\hline Lama3 & Sntg2 & 0.86571 \\
\hline Ces1 & Bcdo2 & 0.73374 \\
\hline
\end{tabular}




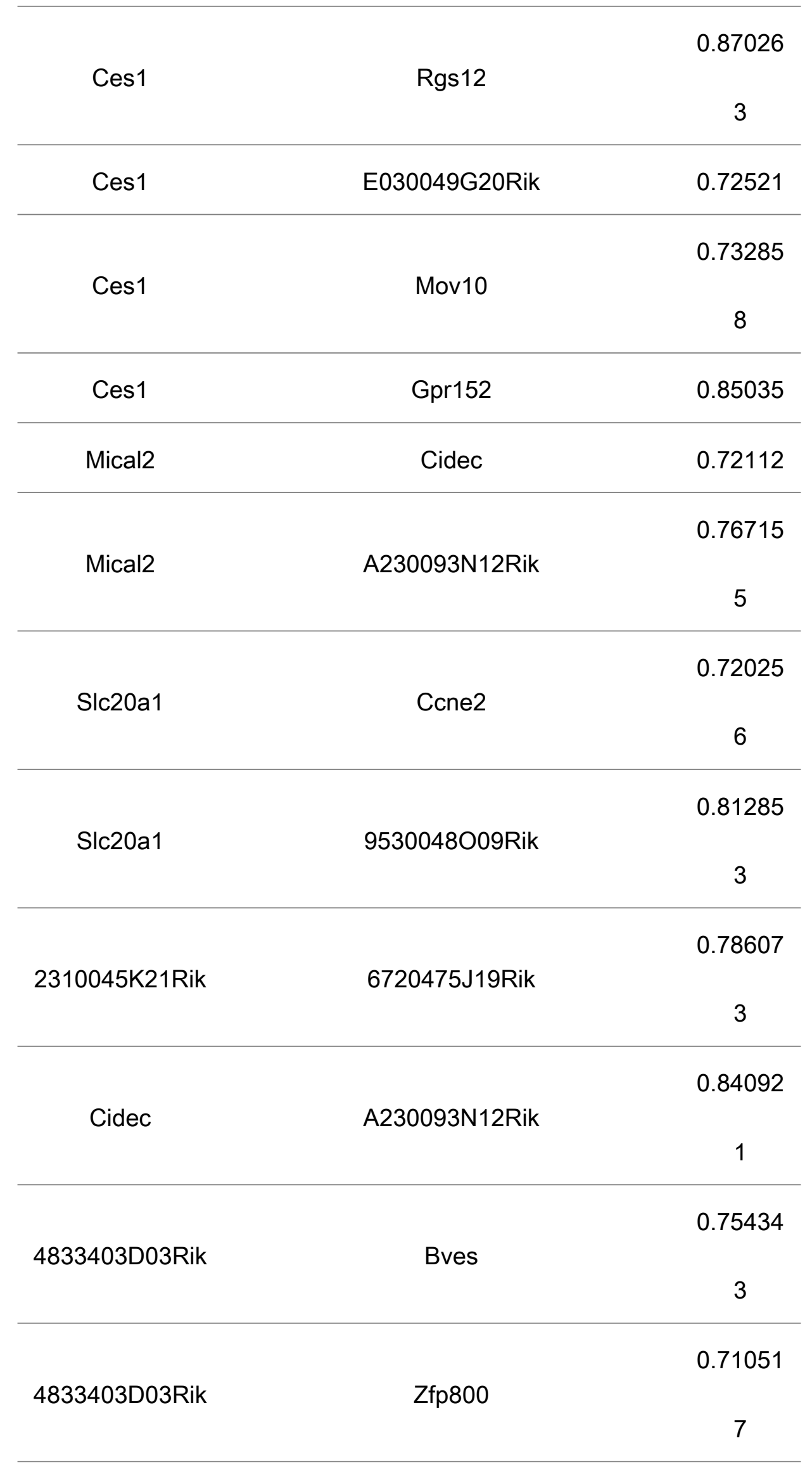




\begin{tabular}{|c|c|c|}
\hline Gins2 & D11Lgp2e & $\begin{array}{c}0.81997 \\
9\end{array}$ \\
\hline \multirow{3}{*}{ Gins2 } & & 0.90602 \\
\hline & 2610042O14Rik & \\
\hline & & 6 \\
\hline \multirow{3}{*}{ Gins2 } & & 0.80629 \\
\hline & B230207L18Rik & \\
\hline & & 7 \\
\hline \multirow{3}{*}{ Gins2 } & & 0.86974 \\
\hline & 1810046K07Rik & \\
\hline & & 9 \\
\hline \multirow{3}{*}{ Gins2 } & & 0.75090 \\
\hline & Gpr152 & \\
\hline & & 7 \\
\hline \multirow[t]{2}{*}{ 1110028F11Rik } & LOC381277 & 0.93156 \\
\hline & & 0.87082 \\
\hline \multirow[t]{3}{*}{ Bves } & 5730410E15Rik & \\
\hline & & 9 \\
\hline & & 0.73172 \\
\hline \multirow[t]{3}{*}{ Grb14 } & Acpp & \\
\hline & & 3 \\
\hline & & 0.98629 \\
\hline \multirow[t]{3}{*}{ Grb14 } & LOC381738 & \\
\hline & & 8 \\
\hline & & 0.72842 \\
\hline \multirow[t]{3}{*}{ Grb14 } & 4632412I06Rik & \\
\hline & & 5 \\
\hline & & 0.75928 \\
\hline \multirow[t]{2}{*}{ Aoc2 } & Srd5a2 & \\
\hline & & 7 \\
\hline
\end{tabular}




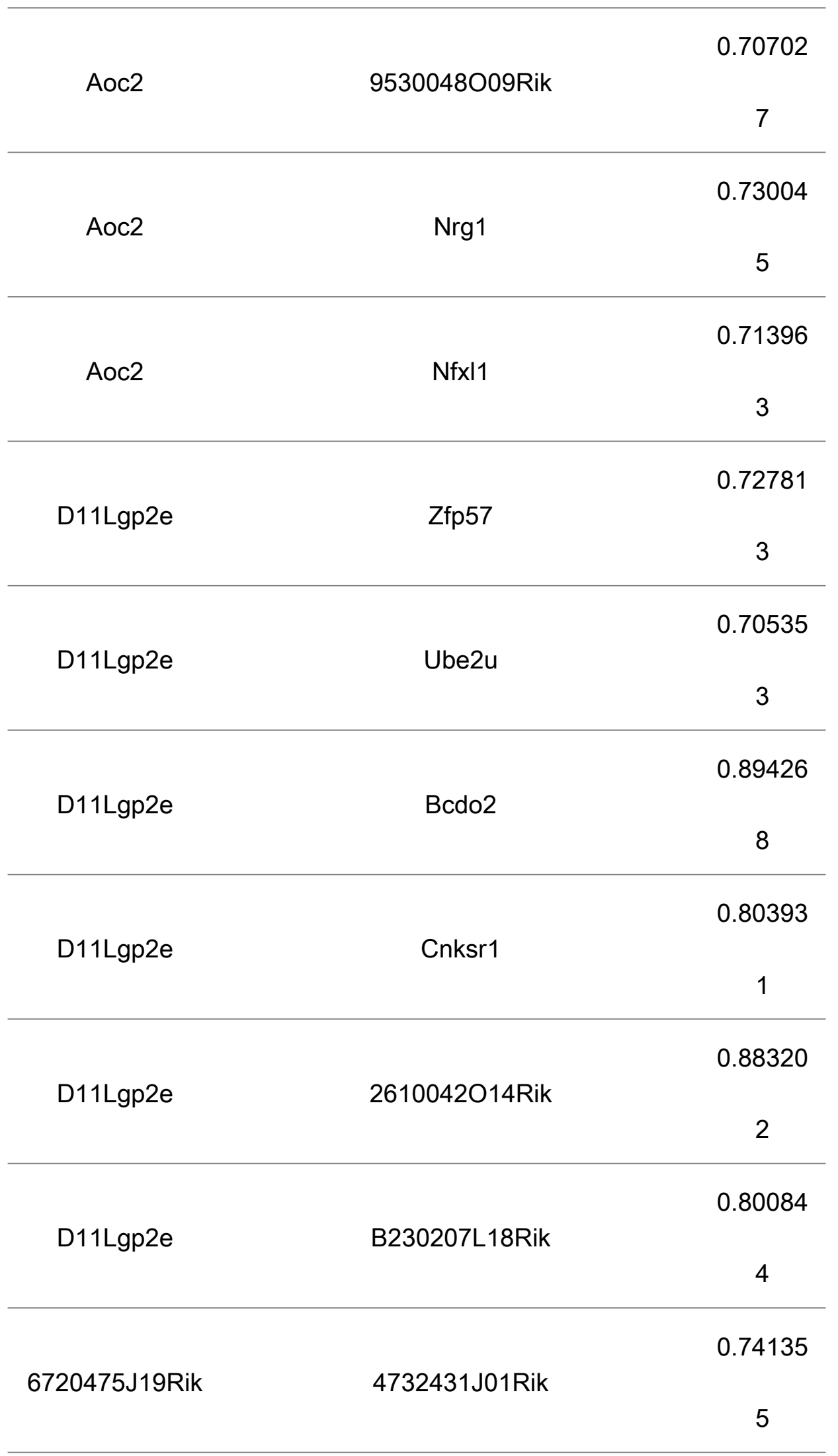




\begin{tabular}{|c|c|c|}
\hline 6720475J19Rik & Gt(ROSA)26asSor & $\begin{array}{c}0.76507 \\
1\end{array}$ \\
\hline LOC212399 & LOC100041004 & $\begin{array}{c}0.70013 \\
2\end{array}$ \\
\hline Scara5 & A530052l19Rik & $\begin{array}{c}0.76726 \\
8\end{array}$ \\
\hline Zfp57 & Bcdo2 & $\begin{array}{c}0.70942 \\
9\end{array}$ \\
\hline Zfp57 & 2610042014Rik & $\begin{array}{c}0.72658 \\
6\end{array}$ \\
\hline Zfp57 & E030049G20Rik & 0.80904 \\
\hline Zfp57 & Mpdz & $\begin{array}{c}0.90999 \\
6\end{array}$ \\
\hline 4930581F22Rik & C730014E05Rik & $\begin{array}{c}0.96805 \\
8\end{array}$ \\
\hline 4930581F22Rik & Rock2 & $\begin{array}{c}0.95907 \\
6\end{array}$ \\
\hline 4930581F22Rik & Wnt5a & $\begin{array}{c}0.79906 \\
8\end{array}$ \\
\hline 4930581F22Rik & A730014O07Rik & $\begin{array}{c}0.76969 \\
5\end{array}$ \\
\hline
\end{tabular}




\begin{tabular}{|c|c|c|}
\hline 1600016N20Rik & LOC243368 & $\begin{array}{c}0.87621 \\
2\end{array}$ \\
\hline LOC383110 & B130065G19Rik & $\begin{array}{c}0.82223 \\
1\end{array}$ \\
\hline Cebpe & C330001K17Rik & $\begin{array}{c}0.70926 \\
7\end{array}$ \\
\hline D930009C14Rik & 1810008K04Rik & $\begin{array}{c}0.82294 \\
2\end{array}$ \\
\hline D930009C14Rik & Nphp3 & 0.78866 \\
\hline D930009C14Rik & 5830411E10Rik & $\begin{array}{c}0.76110 \\
1\end{array}$ \\
\hline D930009C14Rik & Ranbp3I & $\begin{array}{c}0.76709 \\
3\end{array}$ \\
\hline Ube2u & Bcdo2 & $\begin{array}{c}0.70823 \\
6\end{array}$ \\
\hline Ube2u & Cnksr1 & 0.89813 \\
\hline Ube2u & EG666009 & $\begin{array}{c}0.71022 \\
9\end{array}$ \\
\hline Hey2 & D330028D13Rik & $\begin{array}{c}0.80083 \\
8\end{array}$ \\
\hline
\end{tabular}




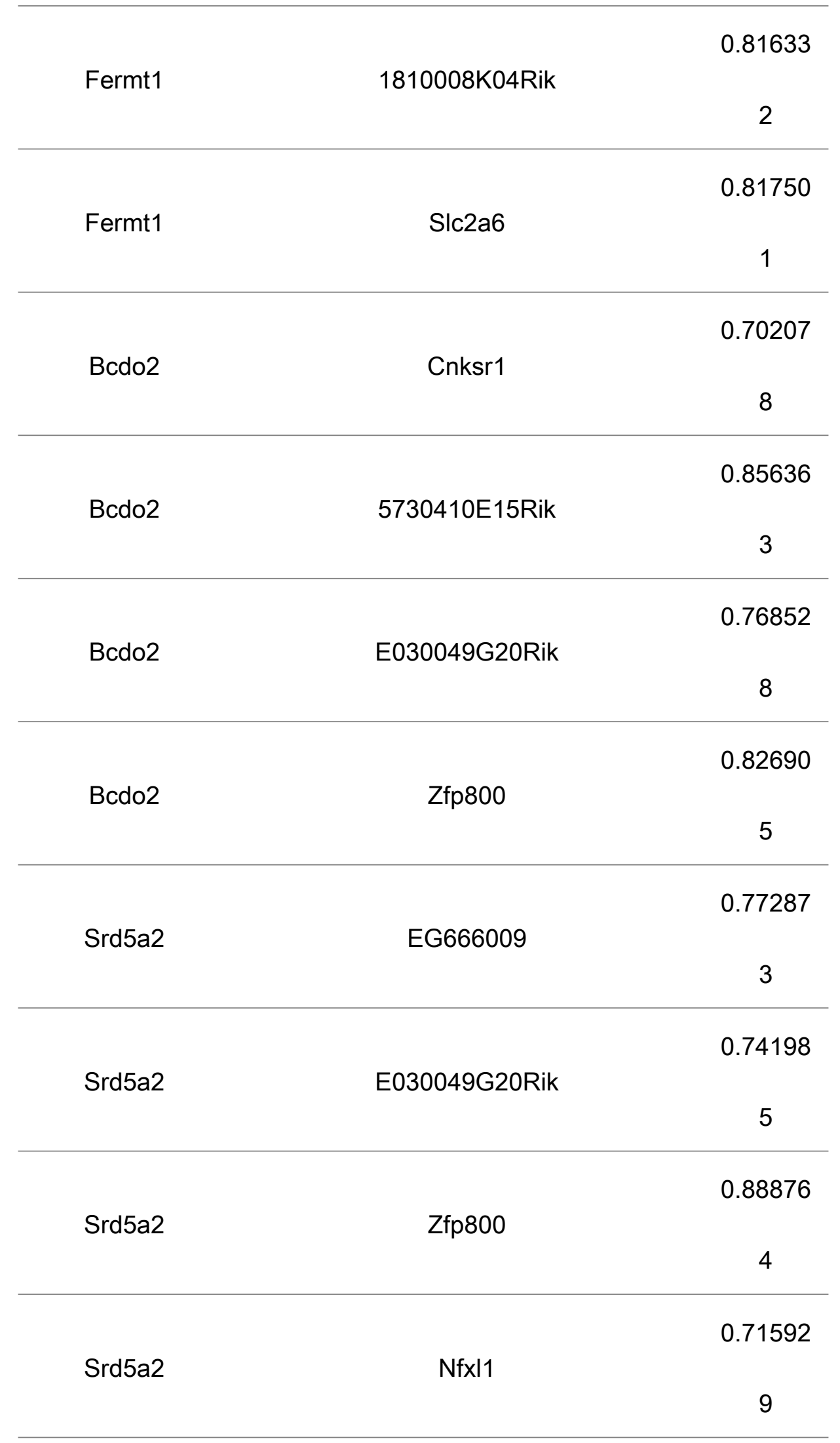




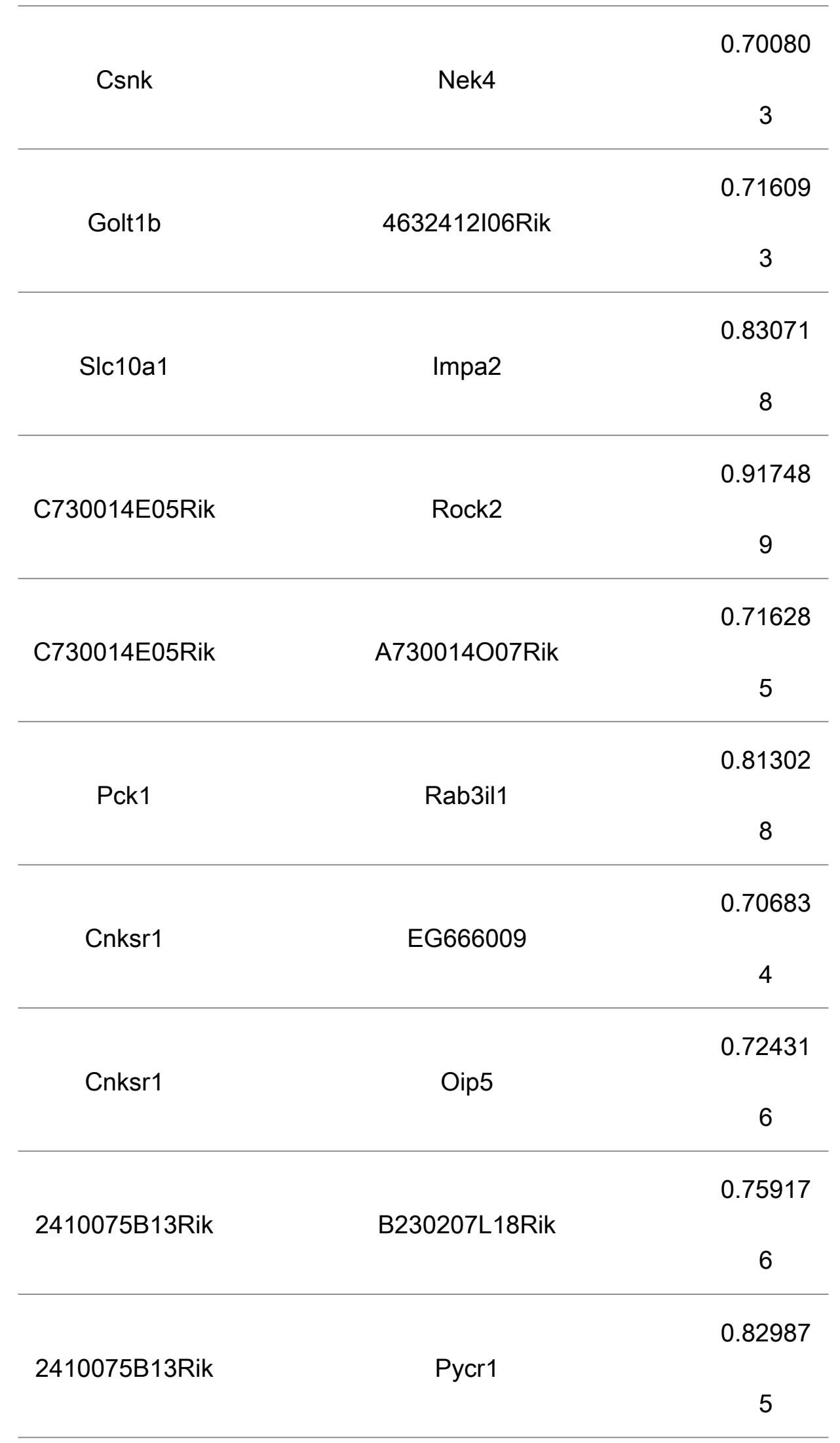




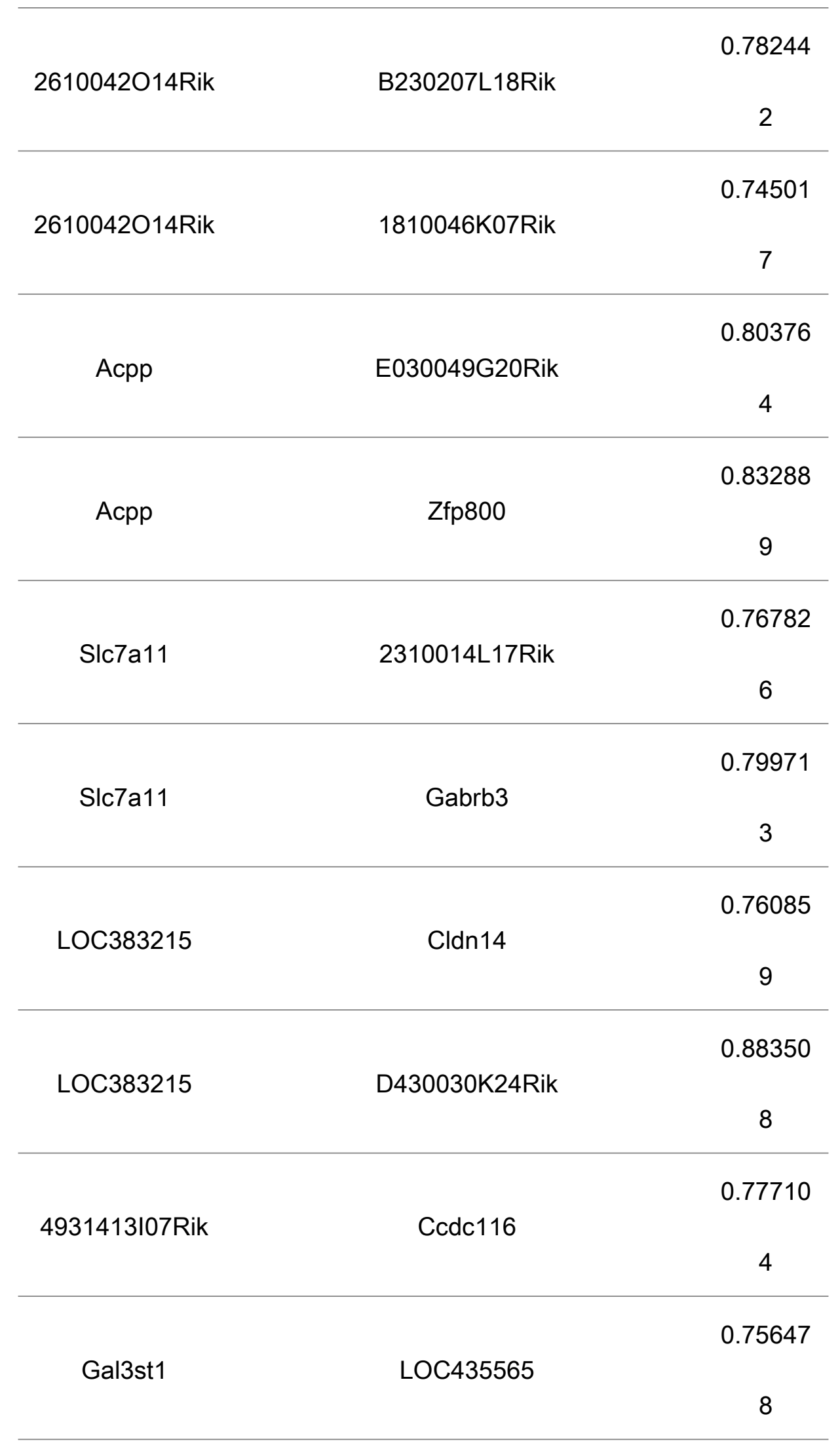




\begin{tabular}{|c|c|c|}
\hline Usf2 & scl0002547.1_9 & $\begin{array}{c}0.79613 \\
2\end{array}$ \\
\hline Usf2 & 4632412I06Rik & 0.70226 \\
\hline Usf2 & Grtp1 & $\begin{array}{c}0.71554 \\
4\end{array}$ \\
\hline Rgs12 & Rock2 & $\begin{array}{c}0.82264 \\
8\end{array}$ \\
\hline Rgs12 & Mov10 & $\begin{array}{c}0.76186 \\
8\end{array}$ \\
\hline Rgs12 & Gpr152 & $\begin{array}{c}0.74324 \\
7\end{array}$ \\
\hline 5730410E15Rik & Nphp3 & 0.72668 \\
\hline 5730410E15Rik & LOC381738 & $\begin{array}{c}0.71802 \\
6\end{array}$ \\
\hline 5730410E15Rik & 5830411E10Rik & 0.7253 \\
\hline 5730410E15Rik & Ranbp3I & $\begin{array}{c}0.70421 \\
7\end{array}$ \\
\hline Rock2 & Wnt5a & 0.83546 \\
\hline 1810008K04Rik & Nphp3 & $\begin{array}{c}0.83854 \\
3\end{array}$ \\
\hline
\end{tabular}




\begin{tabular}{|c|c|c|}
\hline 1810008K04Rik & 5830411E10Rik & $\begin{array}{c}0.85100 \\
1\end{array}$ \\
\hline 1810008K04Rik & Ranbp3I & $\begin{array}{c}0.94938 \\
5\end{array}$ \\
\hline Prkab2 & Wisp2 & $\begin{array}{c}0.76836 \\
7\end{array}$ \\
\hline Prkab2 & Alkbh & $\begin{array}{c}0.82805 \\
6\end{array}$ \\
\hline Nphp3 & LOC381738 & $\begin{array}{c}0.72121 \\
9\end{array}$ \\
\hline Nphp3 & scl0002547.1_9 & $\begin{array}{c}0.83342 \\
7\end{array}$ \\
\hline Nphp3 & 5830411E10Rik & $\begin{array}{c}0.84077 \\
2\end{array}$ \\
\hline Nphp3 & Ranbp3I & $\begin{array}{c}0.93767 \\
5\end{array}$ \\
\hline LOC381738 & Ranbp3| & $\begin{array}{c}0.75770 \\
2\end{array}$ \\
\hline Serpinb1a & Acot3 & $\begin{array}{c}0.79932 \\
8\end{array}$ \\
\hline
\end{tabular}




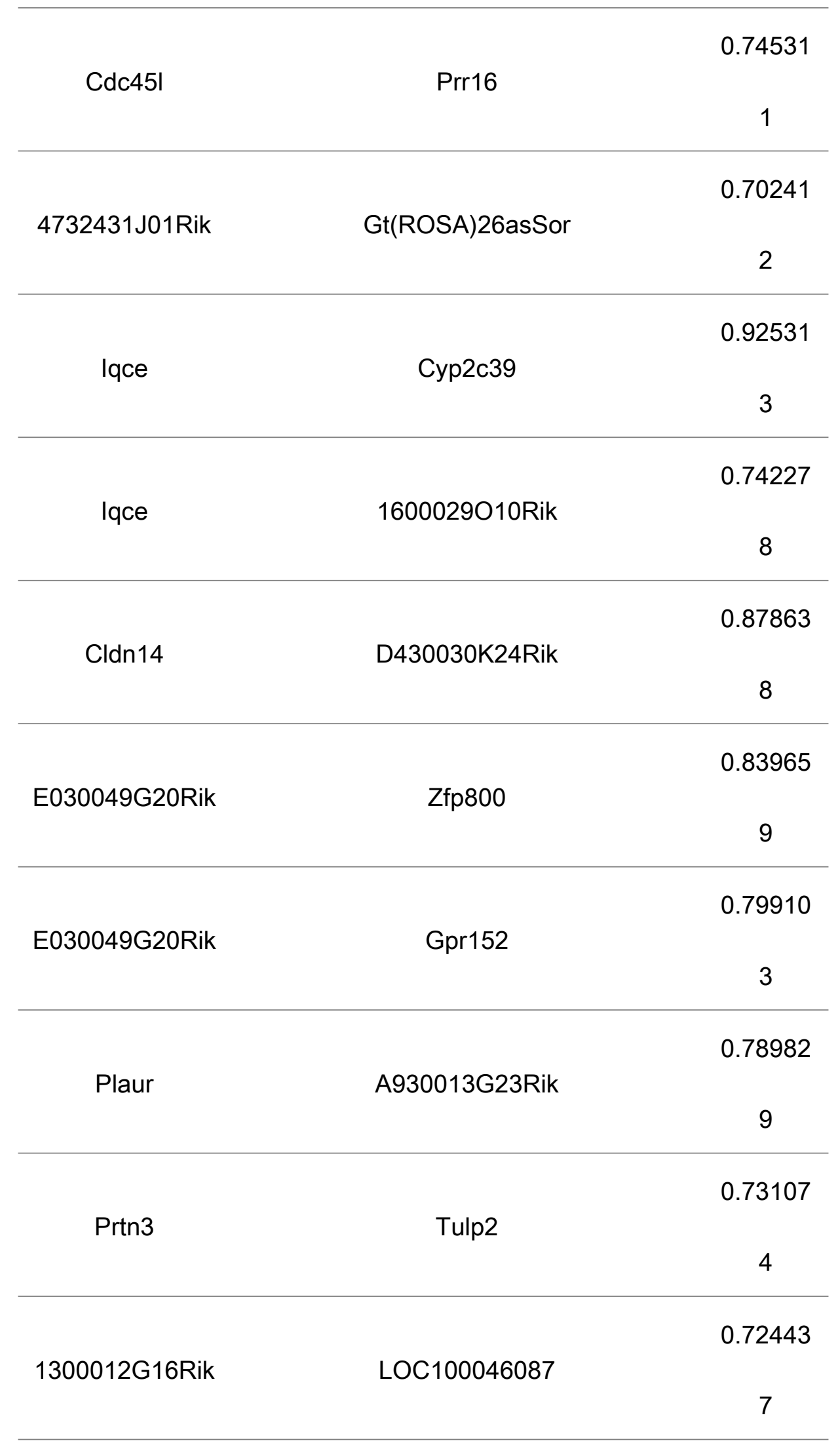




\begin{tabular}{|c|c|c|}
\hline Slc2a6 & A530029H06Rik & $\begin{array}{c}0.74344 \\
2\end{array}$ \\
\hline & & 0.71755 \\
\hline \multirow[t]{3}{*}{ Mov10 } & Gpr152 & \\
\hline & & 7 \\
\hline & & 0.84680 \\
\hline \multirow[t]{3}{*}{ Smpd3 } & EG635702 & \\
\hline & & 1 \\
\hline & IGKV3-2_X16954_Ig_kappa_variable_3- & 0.83618 \\
\hline \multicolumn{3}{|l|}{ Al595366 } \\
\hline & 2_18 & 4 \\
\hline & & 0.71831 \\
\hline \multirow[t]{3}{*}{ B130018F13Rik } & Dhfr & \\
\hline & & 2 \\
\hline & & 0.89575 \\
\hline \multirow[t]{2}{*}{ Kif22 } & Gm379 & \\
\hline & & 7 \\
\hline \multirow[t]{2}{*}{ LOC381277 } & 9330129D05Rik & 0.72746 \\
\hline & & 0.76620 \\
\hline \multirow[t]{3}{*}{ Olfr1043 } & LOC192690 & \\
\hline & & 4 \\
\hline & & 0.86201 \\
\hline \multirow[t]{3}{*}{ Htra4 } & 9130020G22Rik & \\
\hline & & 4 \\
\hline & & 0.79159 \\
\hline \multirow[t]{2}{*}{ Mbd5 } & 4930473A06Rik & \\
\hline & & 2 \\
\hline 9930105H17Rik & 2010007H06Rik & 0.79052 \\
\hline
\end{tabular}




\begin{tabular}{ccc}
\hline 5830411E10Rik & Ranbp3l & 0.89368 \\
\hline Wisp2 & Alkbh & 8 \\
\hline Gt(ROSA)26asS & LOC333825 & 0.7561 \\
or & & 0.77561 \\
Cxxc5 & Armc3 & 5 \\
\hline Fosb & & 0.71439 \\
& & 9 \\
\hline LOC333825 & Mfsd7 & 4 \\
\hline
\end{tabular}


Table S3 Core subnetworks of WGCNA network generated by MCODE plugin.

\begin{tabular}{ccccc}
\hline Cluster & $\begin{array}{c}\text { Score } \\
\text { (Density*\#Nodes) }\end{array}$ & Nodes & Edges & Node IDs \\
\hline 1 & 37.2 & 41 & 744 & $\begin{array}{c}\text { D11Lgp2e, Ces1, Slc41a3, Zfp750, Ngef, LOC433886, Smox, Aox3, } \\
\text { Hsd3b5, Slc25a42, Abca8a, Asns, Fam129a, Dhcr7, Mrp149, Ccdc120, } \\
\text { Acat2, Aldh8a1, Tlcd2, Nalp6, Abcg5, Der13, 2810423A18Rik, EG240327, } \\
\text { Nans, Timd2, Hal, Srm, Syvn1, Pdia4, Creld2, Bcdo2 }\end{array}$ \\
\hline 2 & 9.333 & 10 & 42 & $\begin{array}{c}\text { 4833403D03Rik, Mcf21, Cox6a2, Psat1, C1qtnf1, Zfp800, Kdelr3, } \\
\text { 6720458F09Rik, Srd5a2, Ptrh1 }\end{array}$ \\
\hline 3 & 7.75 & 9 & 31 & EG240549, Prss8, Cdc14b, LOC100047427, Tmie, Cldn1, Cpsf41, \\
EG381806, 2610528J11Rik
\end{tabular}




\begin{tabular}{ccccc}
\hline 13 & 3.2 & 6 & 8 & C730014E05Rik, 4930581F22Rik, Trib3, Cdc42ep5, Ccdc134, Rock2 \\
\hline 14 & 3 & 3 & 3 & Abca2, Selenbp2, Hes6 \\
\hline 15 & 3 & 3 & 3 & Cdkn1a, Amigo3, Nupr1 \\
\hline 16 & 3 & 3 & 3 & Hr, Sdf211, Mthfd2 \\
\hline 17 & 3 & 3 & 3 & Slc17a8, Chrna4, Trim68 \\
\hline 18 & 3 & 3 & 3 & Acacb, Raet1b, Ppargc1b \\
\hline 19 & 3 & 3 & 3 & Irx3, D330028D13Rik, Hey2 \\
\hline 20 & 3 & 3 & 3 & Sntg2, 2010305C02Rik, Lama3 \\
\hline 21 & 3 & 3 & 3 & B430114K07Rik, 1600016N20Rik, LOC243368 \\
\hline 22 & 3 & 3 & 3 & Dbp, Cyp2c39, Iqce \\
\hline 23 & 3 & 3 & 3 & LOC211591, Vwce, Nr1d1 \\
\hline 24 & 3 & 3 & 3 & LOC100044164, Mapk15, Clstn3 \\
\hline 25 & 3 & 3 & 3 & Il12rb1, Gt(ROSA)26asSor, 6720475J19Rik \\
\hline 26 & 3 & 3 & 3 & Atf3, Alkbh, Csn3 \\
\hline 27 & 3 & 3 & 3 &
\end{tabular}

Table S4 Core subnetworks of PPI network generated by MCODE plugin.

\begin{tabular}{ccccc}
\hline Cluster & $\begin{array}{c}\text { Score } \\
\text { (Density*\#Nodes) }\end{array}$ & Nodes & Edges & Node IDs \\
\hline 1 & 11 & 13 & 66 & Sdf211, Dnajc3, Creld2, Manf, Der11, Der13, Dnajb9, Sel11, Syvn1, Pdia4, \\
Hyou1, Hspa5, Ddit3 \\
\hline 2 & 6 & 6 & 15 & Gng13, Mtnr1a, Sucnr1, Gpsm2, P2ry4, Ptgdr2 \\
\hline 3 & 4.5 & 5 & 9 & Parp14, Ifi47, Dhx58, Oas11, Isg20 \\
\hline 4 & 4.4 & 6 & 11 & Rad54b, Kif22, Cdc45, Ccne2, Gins2, Oip5 \\
\hline 5 & 4 & 4 & 6 & Drc1, Dyx1c1, Dnaic1, Ccdc151 \\
\hline
\end{tabular}




\begin{tabular}{ccccc}
\hline 6 & 3.429 & 8 & 12 & Acss2, Thrsp, Acacb, Aldh18a1, Pycr1, Acat2, Mthfd2, Psat1 \\
\hline 7 & 3.333 & 4 & 5 & Atp8b1, Abcg8, Abcg5, Dhcr7 \\
\hline 8 & 3 & 3 & 3 & Upp2, B3gat2, Bdh1 \\
\hline 9 & 3 & 3 & 3 & Fbx116, Asb2, Ube2u \\
\hline 10 & 3 & 3 & 3 & P2ry2, Tfec, Cebpe \\
\hline 11 & 3 & 3 & 3 & Unc93a, Mfsd7a, Mfsd9 \\
\hline 12 & 3 & 3 & 3 & Trmt61a, Nop56, Rrs1 \\
\hline
\end{tabular}

Table S5 Differentially expressed classes of liver metabolites (normalized).

\begin{tabular}{|c|c|c|c|c|c|c|c|c|c|c|c|c|}
\hline Class & $\mathrm{C} 1$ & $\mathrm{C} 2$ & $\mathrm{C} 3$ & $\mathrm{C} 4$ & $\mathrm{C} 5$ & C6 & $\mathrm{T} 1$ & $\mathrm{~T} 2$ & $\mathrm{~T} 3$ & $\mathrm{~T} 4$ & $\mathrm{~T} 5$ & $\mathrm{~T} 6$ \\
\hline Acyl carnitines & 0 & 0.070106 & 0.202214 & 0.268734 & 0.570143 & 0.191746 & 0.698852 & 0.228212 & 1 & 0.437573 & 0.256639 & 0.470583 \\
\hline Amino acids & 0.414571 & 0.031411 & 0.606199 & 0.425946 & 0.666555 & 0.592953 & 0.631164 & 0.328466 & 1 & 0 & 0.015072 & 0.334929 \\
\hline Aminoxides & 0.355416 & 0.067127 & 0.582794 & 0.219437 & 1 & 0.418692 & 0.017896 & 0.010754 & 0 & 0.051372 & 0.030903 & 0.150408 \\
\hline Benzene & 0.490098 & 0.779614 & 1 & 0.57948 & 0.448758 & 0.846245 & 0.186153 & 0.299595 & 0 & 0.263589 & 0.473161 & 0.211214 \\
\hline Benzoic acids & 0.396918 & 0.121726 & 0.316534 & 0.323311 & 0.435207 & 0.234147 & 1 & 0.069552 & 0.028418 & 0 & 0.03252 & 0.139654 \\
\hline Bile acids & 0.421536 & 0 & 0.259273 & 0.155311 & 0.35144 & 0.086207 & 0.458233 & 0.369649 & 0.478618 & 0.468968 & 0.935333 & 1 \\
\hline Carbohydrates & 0.502353 & 0.672819 & 0.789464 & 0.900527 & 1 & 0.992287 & 0.216547 & 0.353602 & 0 & 0.170637 & 0.106505 & 0.372918 \\
\hline Carboxylic acids & 0.178488 & 0.131139 & 0.424021 & 0.387878 & 0.574076 & 0.262031 & 0.22387 & 0 & 0.293027 & 0.694762 & 1 & 0.629317 \\
\hline Fatty acids & 1 & 0.356181 & 0 & 0.315029 & 0.507805 & 0.625991 & 0.403854 & 0.405626 & 0.120436 & 0.387147 & 0.150626 & 0.70943 \\
\hline Fatty Acyls & 0.324162 & 1 & 0.366294 & 0 & 0.328461 & 0.371453 & 0.05331 & 0.793637 & 0.069647 & 0.638005 & 0.439381 & 0.558899 \\
\hline Glycerophosphocholines & 1 & 0.432527 & 0.21481 & 0.325712 & 0.5732 & 0.931546 & 0 & 0.335702 & 0.09796 & 0.190199 & 0.142474 & 0.430234 \\
\hline $\begin{array}{l}\text { Glycerophosphoethanola } \\
\text { mines }\end{array}$ & 1 & 0.266935 & 0.074026 & 0.626494 & 0.342834 & 0.722924 & 0.230243 & 0 & 0.070236 & 0.111322 & 0.166557 & 0.051629 \\
\hline Glycerophosphoglycerol & 0.728556 & 0.663409 & 0.90228 & 0.913138 & 0.674267 & 1 & 0 & 0.196526 & 0.402823 & 0.083605 & 0.064061 & 0.337676 \\
\hline
\end{tabular}




\begin{tabular}{|c|c|c|c|c|c|c|c|c|c|c|c|c|}
\hline Hydroxy acids & 0.396832 & 0.260062 & 0.188863 & 0.298761 & 0.634468 & 0.235441 & 0 & 0.299676 & 0.268484 & 0.301613 & 1 & 0.481782 \\
\hline Imidazoles & 0.201531 & 0.122449 & 0.428571 & 0.352041 & 0.181122 & 0.423469 & 1 & 0.239796 & 0.737245 & 0.071429 & 0 & 0.158163 \\
\hline Indoles & 0.191022 & 0.091846 & 0.026179 & 0.861901 & 0.45432 & 0.346568 & 0.668132 & 0.91462 & 1 & 0.668779 & 0 & 0.25017 \\
\hline Indolyl carboxylic acids & 0.215027 & 0 & 0.537335 & 0.586716 & 0.589645 & 1 & 0.666578 & 0.802742 & 0.837615 & 0.842207 & 0.556036 & 0.611141 \\
\hline Keto acids & 0.462963 & 1 & 0.953676 & 0.516576 & 0.454397 & 0.933826 & 0.21029 & 0.264214 & 0 & 0.382359 & 0.735061 & 0.333289 \\
\hline Nucleosides & 0 & 0.30149 & 0.685051 & 0.695021 & 0.361148 & 1 & 0.699338 & 0.678001 & 0.350244 & 0.327039 & 0.111723 & 0.585721 \\
\hline Organic phosphoric acids & 0.022793 & 0 & 0.341893 & 0.212734 & 0.303905 & 0.3191 & 0.70658 & 0.415887 & 1 & 0.57742 & 0.41787 & 0.197538 \\
\hline Organic sulfuric acids & 0 & 0.132881 & 0.376116 & 0.427739 & 0.813767 & 0.671999 & 1 & 0.613489 & 0.766769 & 0.592878 & 0.320269 & 0.231353 \\
\hline Organooxygen & 0.857065 & 0.591493 & 0.388925 & 0.742717 & 0.928533 & 1 & 0.6053 & 0.586633 & 0.077456 & 0.357137 & 0 & 0.477116 \\
\hline Phenols & 0 & 0.098546 & 0.097082 & 0.261959 & 0.156145 & 0.134234 & 0.507322 & 0.625339 & 1 & 0.659833 & 0.493166 & 0.416368 \\
\hline Phenylpropanoic acids & 0.355493 & 0.480886 & 0.350149 & 0.907363 & 0.890998 & 1 & 0.244593 & 0.318303 & 0.190078 & 0.373653 & 0 & 0.307709 \\
\hline Phosphosphingolipids & 0.020654 & 0.193757 & 0.261253 & 0.456994 & 0.43041 & 0.489089 & 1 & 0.172905 & 0.926773 & 0.56862 & 0 & 0.397324 \\
\hline Purine nucleosides & 0.983297 & 0 & 0.139553 & 0.167096 & 0.37908 & 0.751431 & 0.557955 & 0.874315 & 0.252196 & 0.552237 & 1 & 0.398738 \\
\hline Purines & 0.715737 & 0.148473 & 0 & 0.133018 & 0.498249 & 0.563081 & 0.594598 & 0.588755 & 0.436807 & 0.764953 & 1 & 0.541748 \\
\hline Pyridines & 0.594564 & 0.243826 & 0.211638 & 0.343582 & 0 & 0.724193 & 0.199646 & 0.182891 & 0.287877 & 0.24175 & 0.203032 & 1 \\
\hline Pyrimidine nucleosides & 0.993346 & 0.274451 & 0 & 0.128391 & 0.42845 & 0.903684 & 0.645408 & 1 & 0.141691 & 0.839646 & 0.451711 & 0.786153 \\
\hline Pyrimidine nucleotides & 0.46696 & 0 & 0.096916 & 0.044053 & 0.462555 & 0.251101 & 0.427313 & 0.052863 & 0.242291 & 0.286344 & 1 & 0.400881 \\
\hline Pyrimidines & 0.009892 & 0 & 0.027704 & 0.021635 & 0.124493 & 0.444433 & 0.869892 & 0.517379 & 0.788729 & 1 & 0.538522 & 0.560906 \\
\hline $\begin{array}{c}\text { Quaternary ammonium } \\
\text { salts }\end{array}$ & 0.14978 & 0 & 0.116892 & 0.242215 & 0.124639 & 0.111727 & 0.775254 & 0.714408 & 0.17828 & 0.931186 & 0.982531 & 1 \\
\hline $\begin{array}{l}\text { Quinoline carboxylic } \\
\text { acids }\end{array}$ & 0.376901 & 0.556271 & 0.244931 & 0.728427 & 0.575233 & 1 & 0.211353 & 0.332293 & 0.125982 & 0.170241 & 0 & 0.164197 \\
\hline
\end{tabular}

Table S6 Differentially expressed glycerophosphoglycerols, glycerophosphocholines and glycerophosphoethanolamines (normalized).

\begin{tabular}{llllllllllllll}
\hline Metabolites & $\mathrm{C} 1$ & $\mathrm{C} 2$ & $\mathrm{C} 3$ & $\mathrm{C} 4$ & $\mathrm{C} 5$ & $\mathrm{C} 6$ & $\mathrm{~T} 1$ & $\mathrm{~T} 2$ & $\mathrm{~T} 3$ & $\mathrm{~T} 4$ & $\mathrm{~T} 5$ & $\mathrm{~T} 6$ & \\
\hline
\end{tabular}




\begin{tabular}{|c|c|c|c|c|c|c|c|c|c|c|c|c|}
\hline Glycerophosphocholine & .589635 & 0.645709 & 0.749363 & 0.637213 & 0.68989 & 0.55565 & 0 & 0.330702 & 1 & 0.254885 & 0.33305 & 0.183517 \\
\hline LysoPC(18:1) & 1 & 0.463506 & 5165 & 94885 & 0.532127 & 0.675608 & 0 & 0.270983 & 1591 & .145352 & 399 & 0.22645 \\
\hline LysoPC(14:0) & 710083 & 0.539074 & 0.447135 & 0.452651 & 0.733681 & 1 & 0 & 0.277413 & 0.205945 & 0.1327 & 0.236899 & 0.580754 \\
\hline LysoPC(16:0) & 1 & 0.723145 & 0.258029 & 0.379845 & 0.645626 & 0.933555 & 0.158361 & 0.722038 & 0 & 0.390919 & 0.202658 & 0.291251 \\
\hline LysoPC(16:1) & 826389 & 0.727183 & 0.627976 & 0.460565 & 0.795387 & 1 & 0 & 0.496298 & 0.205915 & 0.30692 & 0.398562 & 0.479167 \\
\hline LysoPC(18:2) & 1 & 0.280419 & 0.188836 & 0.402529 & 0.328391 & 0.847362 & 0.171391 & 0.264474 & 0 & 0.258613 & 0.12778 & 0.297863 \\
\hline LysoPC(18:3) & 1 & 0.694215 & 0.14876 & 0.512397 & 0.429752 & 0.247934 & 0.404959 & 0.357438 & 0.065289 & 0.289256 & 0 & 0.016529 \\
\hline LysoPC(20:4) & 1 & 0 & 0.052738 & 0.515213 & 0.273834 & 0.585193 & 0.314402 & 0.057593 & 0.321501 & 0.154158 & 0.154158 & 0.295132 \\
\hline LysoPC(20:5) & 1 & 0.353978 & 0.171407 & 0.459308 & 0.283758 & 0.571659 & 0.192472 & 0.270227 & 0 & 0.241626 & 0.031529 & 0.087143 \\
\hline LysoPC (22:4) & 0.089556 & 0.317222 & 0.433556 & 0.258444 & 0.634333 & 0.652333 & 0 & 0.171444 & 0.083333 & 1 & 0.328222 & 0.572889 \\
\hline LysoPC(22:5) & 1 & 0 & 0.067711 & 0.89351 & 0.474248 & 0.803094 & 0.446119 & 0.268301 & 0.187596 & 0.15076 & 0.164155 & 0.554618 \\
\hline LysoPC(22:6) & 1 & 0 & 0.059976 & 0.611995 & 0.247246 & 796 & 0.580171 & 294 & 0.397552 & 0.235006 & 0.3 & 0.432069 \\
\hline LysoPC(18:0) & 0.015129 & 0.145234 & 0.413011 & 0 & 0.236006 & 0.102874 & 0.629349 & 0.263255 & 0.271558 & 0.337368 & 0.531014 & 1 \\
\hline PC (16:0) & 1 & 0.406297 & 0 & 0.303935 & 0.303935 & 0.651967 & 0.324407 & 0.717582 & 0.477951 & 0.42677 & 0.467715 & 0.087377 \\
\hline sn2 LysoPC(22:5) & 0.610767 & 0.004768 & 0.097088 & 0.891786 & 0.615502 & 1 & 0.520476 & 0 & 0.20395 & 0.402455 & 0.638497 & 0.679077 \\
\hline sn2 LysoPC(18:2) & 0.772645 & 0.220498 & 0.018405 & 0.447853 & 0.379285 & 1 & 0.18441 & 0.2831 & 0 & 0.451462 & 0.361241 & 0.411765 \\
\hline sn2 LysoPC(20:4) & 1 & 0 & 0.001638 & 0.73792 & 0.52498 & 0.96724 & 0.306306 & 0.029419 & 0.438984 & 0.25389 & 0.369369 & 0.418509 \\
\hline sn2 LysoPC(14:0) & 0.714489 & 0.524148 & 0.517045 & 0.447727 & 0.961364 & 1 & 0 & 0.914205 & 0.354545 & 0.290057 & 0.276989 & 0.844602 \\
\hline sn2 LysoPC(20:5) & 1 & 0.4196 & 0.03901 & 0.647954 & 0.476689 & 0.809705 & 0.362512 & 0.358887 & 0 & 0.647954 & 0.248335 & 0.23882 \\
\hline sn2 LysoPC(16:0) & 0.69475 & 0.474969 & 0.316239 & 0.218559 & 0.6337 & 1 & 0 & 0.372851 & 0.021978 & 0.316239 & 0.023199 & 0.389499 \\
\hline sn2 LysoPC(18:3) & 1 & 0.767654 & 0.470225 & 0.410348 & 0.683697 & 0.924178 & 0.303938 & 0.482167 & 0.218028 & 0.487472 & 0 & 0.297104 \\
\hline sn2 LysoPC(18:1) & 0.908616 & 0.738903 & 0.360313 & 0.660574 & 0.921671 & 1 & 0 & 0.367027 & 0.18799 & 0.386423 & 0.295039 & 0.543081 \\
\hline sn2 LysoPC(16:1) & 0.452976 & 0.720956 & 0.678026 & 0.547909 & 1 & 0.795087 & 0 & 0.361983 & 0.164196 & 0.365789 & 0.362027 & 0.386147 \\
\hline LysoPE(16:0) & 1 & 0.47493 & 0.057103 & 0.554318 & 0.263231 & 0.651811 & 0.454039 & 0 & 0.044568 & 0.182451 & 0.126741 & 0.026462 \\
\hline LysoPE(20:4) & 1 & 0.070408 & 0.054594 & 0.55167 & 0.197082 & 0.555746 & 0.147521 & 0 & 0.054513 & 0.035601 & 0.10387 & 0.047829 \\
\hline
\end{tabular}




\begin{tabular}{|c|c|c|c|c|c|c|c|c|c|c|c|c|}
\hline LysoPE(20:5) & 1 & 0.044603 & 0.026916 & 0.639949 & 0.114507 & 0.499579 & 0.14595 & 0 & 0.016143 & 0.009966 & 0.029337 & 0.035794 \\
\hline LysoPE(16:1) & 1 & 0.599946 & 0.387711 & 0.520392 & 0.682184 & 0.663134 & 0.083445 & 0 & 0.072176 & 0.129192 & 0.25436 & 0.178696 \\
\hline LysoPE(18:1) & 1 & 0.362903 & 0.076613 & 0.548387 & 0.483871 & 0.790323 & 0.056452 & 0.004117 & 0.106452 & 0.060484 & 0.120968 & 0 \\
\hline LysoPE(18:2) & 1 & 0.256322 & 0.109136 & 0.526956 & 0.22209 & 0.844943 & 0.125358 & 0.036987 & 0 & 0.106512 & 0.159351 & 0.004532 \\
\hline LysoPE(22:6) & 1 & 0.041367 & 0.082714 & 0.71502 & 0.253902 & 0.56503 & 0.254202 & 0 & 0.168008 & 0.065365 & 0.159959 & 0.077815 \\
\hline sn2 LysoPE(16:0) & 1 & 0.317068 & 0.093946 & 0.530472 & 0.260174 & 0.610447 & 0.520348 & 0 & 0.186475 & 0.226159 & 0.186273 & 0.111359 \\
\hline sn2 LysoPE(22:6) & 1 & 0.052608 & 0.072142 & 0.856552 & 0.451521 & 0.81858 & 0.215253 & 0 & 0.183947 & 0.110114 & 0.291196 & 0.125977 \\
\hline sn2 LysoPE(20:4) & 1 & 0.09914 & 0.016204 & 0.671115 & 0.408006 & 0.825548 & 0.059102 & 0 & 0.020494 & 0.087701 & 0.236414 & 0.102 \\
\hline sn2 LysoPE(20:5) & 1 & 0.099552 & 0.035117 & 0.853705 & 0.257412 & 0.829373 & 0.128241 & 0 & 0.011085 & 0.06759 & 0.086425 & 0.044309 \\
\hline sn2 LysoPE(18:1) & 0.535396 & 0.673833 & 0.416361 & 0.809649 & 0.840587 & 1 & 0.292606 & 0.261563 & 0.457263 & 0.415312 & 0.481384 & 0 \\
\hline sn2 LysoPE(18:2) & 0.902232 & 0.34349 & 0.178426 & 0.657813 & 0.460974 & 1 & 0.059883 & 0.038529 & 0 & 0.108359 & 0.198142 & 0.021509 \\
\hline sn2 LysoPE(16:1) & 1 & 0.579729 & 0.371829 & 0.569824 & 0.752356 & 0.777845 & 0 & 0.000604 & 0.112708 & 0.128775 & 0.223605 & 0.086615 \\
\hline $1-$ & 0.728556 & 0.663409 & 0.90228 & 0.913138 & 0.674267 & 1 & 0 & 0.196526 & 0.402823 & 0.083605 & 0.064061 & 0.337676 \\
\hline $\begin{array}{l}\text { Stearoylglycerophospho- } \\
\text { glycerol }\end{array}$ & & & & & & & & & & & & \\
\hline
\end{tabular}

Table S7 Differentially expressed glycerophosphoglycerols, glycerophosphocholines and glycerophosphoethanolamines (normalized).

\begin{tabular}{lrrrrrrrrrrrrrrr}
\hline Metabolites & C1 & C2 & C3 & C4 & C5 & C6 & T1 & T2 & T3 & T4 & T5 & T6 & \\
\hline 1-Methylhistidine & 0.094633 & 0 & 0.157631 & 0.172219 & 0.17002 & 0.062656 & 0.981286 & 0.447256 & 0.391441 & 1 & 0.647748 & 0.428854 \\
\hline Betaine & 0.04809 & 0 & 0.399104 & 0.382202 & 0.530675 & 0.320198 & 0.482585 & 0.408868 & 1 & 0.16939 & 0.105892 & 0.374638 \\
\hline Creatine & 0.595554 & 0 & 0.09094 & 0.126258 & 1 & 0.017158 & 0.543754 & 0.365825 & 0.919147 & 0.415508 & 0.234967 & 0.182816 \\
\hline Glycine & 0.034188 & 0.093629 & 0.969308 & 1 & 0.461538 & 0.904429 & 0.29798 & 0.027483 & 0 & 0.441595 & 0.138695 & 0.117586 \\
\hline Glutathione & 1 & 0.703713 & 0.808247 & 0.969423 & 0.764177 & 0.890677 & 0.513433 & 0.422806 & 0.77113 & 0 & 0.033414 & 0.580897 \\
\hline L-Glutamic acid & 0.382743 & 0.498157 & 0.030573 & 0 & 0.51081 & 0.267232 & 0.393608 & 0.022431 & 0.630597 & 0.970294 & & 0.299455 \\
\hline L-Tyrosine & 0.85979 & 0.303497 & 0.35979 & 0.464802 & 0.49965 & & 1 & 0 & 0.592421 & 0.198252 & 0.356993 & 0.419347 & 0.276457 \\
\hline
\end{tabular}




\begin{tabular}{|c|c|c|c|c|c|c|c|c|c|c|c|c|}
\hline L-Phenylalanine & 1 & 0.623131 & 0.572782 & 0.755234 & 0.998172 & 0.694084 & 0.22333 & 0.316491 & 0.604021 & 0.170987 & 0 & 0.421402 \\
\hline L-Alanine & 1 & 0.666113 & 0.874585 & 0.790282 & 0.775748 & 0.982973 & 0.784884 & 0.592869 & 0.096761 & 0.605897 & 0 & 0.489618 \\
\hline L-Proline & 0.365115 & 0.863584 & 0.779643 & 0.91606 & 0.291205 & 1 & 0.011298 & 0.523562 & 0.072432 & 0.440925 & 0.166508 & 0 \\
\hline L-Asparagine & 1 & 0.845013 & 0.38505 & 0.407222 & 0.678834 & 0.569226 & 0 & 0.310335 & 0.179081 & 0.168842 & 0.361153 & 0.369437 \\
\hline L-Isoleucine & 0.693315 & 0.257453 & 0.51626 & 0.230352 & 0 & 0.67841 & 1 & 0.798555 & 0.209124 & 0.361337 & 0.015808 & 0.320235 \\
\hline L-Histidine & 0.260823 & 0.206065 & 1 & 0.296181 & 0 & 0.730613 & 0.839706 & 0.332599 & 0.11237 & 0.727313 & 0.548556 & 0.406117 \\
\hline L-Lysine & 0.3478 & 0 & 0.461902 & 0.265692 & 0.01815 & 0.278407 & 0.768113 & 1 & 0.422779 & 0.719603 & 0.373199 & 0.303716 \\
\hline L-Serine & 0.689927 & 0.373849 & 0.731154 & 0.96531 & 0.863109 & 1 & 0 & 0.686045 & 0.29046 & 0.414276 & 0.972382 & 0.303402 \\
\hline L-Aspartic acid & 1 & 0.719151 & 0.247828 & 0.175755 & 0.473115 & 0.59017 & 0 & 0.079484 & 0.379188 & 0.308355 & 0.903971 & 0.200882 \\
\hline Ornithine & 0.117809 & 0.121644 & 0 & 0.433649 & 0.00147 & 0.261634 & 0.341665 & 1 & 0.703401 & 0.582843 & 0.208514 & 0.024163 \\
\hline Taurine & 0 & 0.145552 & 0.54679 & 0.481862 & 0.2856 & 0.4241 & 0.940518 & 0.856383 & 1 & 0.908269 & 0.904542 & 0.86097 \\
\hline Pyroglutamic acid & 0.188544 & 0.104698 & 0.006909 & 0 & 0.156921 & 0.165588 & 1 & 0.600835 & 0.206727 & 0.528922 & 0.447934 & 0.380291 \\
\hline Sarcosine & 0.568607 & 0 & 0.044677 & 0.083654 & 0.962879 & 0.111759 & 0.548588 & 0.24208 & 1 & 0.430996 & 0.246984 & 0.144637 \\
\hline Urea & 0.294494 & 0.510162 & 0.595608 & 0.921967 & 0.706698 & 0.845937 & 0.696481 & 0.685669 & 0 & 0.708401 & 1 & 0.513468 \\
\hline 5-Hydroxy-L-tryptophan & 0.985035 & 0.308807 & 0.030553 & 0.332502 & 0.568667 & 0.835698 & 0.379891 & 0.083866 & 0.706313 & 0.177553 & 0 & 1 \\
\hline L-Arginine & 0.284675 & 0.531238 & 0.48251 & 0.491729 & 1 & 0.229582 & 0.20734 & 0 & 0.358424 & 0.051281 & 0.030283 & 0.201267 \\
\hline L-Glutamine & 0.343154 & 0.292695 & 0.301589 & 0 & 0.03171 & 0.177148 & 1 & 0.345805 & 0.174697 & 0.819365 & 0.491093 & 0.603027 \\
\hline L-Leucine & 0.935733 & 0.478149 & 0.498715 & 0.22108 & 0.210797 & 1 & 0.838046 & 0.735219 & 0 & 0.565553 & 0.596401 & 0.264781 \\
\hline L-Methionine & 1 & 0.92957 & 0.67035 & 0.654572 & 0.548002 & 0.834221 & 0.571441 & 0.990609 & 0 & 0.900834 & 0.635634 & 0.508473 \\
\hline $\begin{array}{l}\text { L-Aspartyl-L- } \\
\text { phenylalanine }\end{array}$ & 0 & 0.177938 & 0.419535 & 0.358954 & 0.14052 & 0.300559 & 0.876407 & 0.674739 & 0.197214 & 0.761157 & 1 & 0.208877 \\
\hline 4-Hydroxyproline & 0.334387 & 0.379674 & 0.78673 & 1 & 0.956819 & 0.398631 & 0.424961 & 0.579379 & 0 & 0.769879 & 0.04476 & 0.066877 \\
\hline N-Acetyl-L-alanine & 0.29943 & 0.070129 & 0.291339 & 0.133713 & 0.2343 & 0.272466 & 0.354481 & 0.535075 & 0 & 0.001185 & 1 & 0.102594 \\
\hline L-Valine & 0.575939 & 0.25367 & 0.595351 & 0 & 0.195315 & 1 & 0.668204 & 0.928345 & 0.135642 & 0.622431 & 0.053562 & 0.022767 \\
\hline Citrulline & 0.379198 & 0.565377 & 0.446677 & 1 & 0.542373 & 0.668435 & 0.38656 & 0.29209 & 0.189032 & 0.0899 & 0 & 0.095237 \\
\hline
\end{tabular}




\begin{tabular}{|c|c|c|c|c|c|c|c|c|c|c|c|c|}
\hline L-Tryptophan & 0.32409 & 0 & 0.126311 & 0.299905 & 0.358836 & 0.445292 & 0.191443 & 0.466617 & 0.686946 & 0.607508 & 1 & 0.824499 \\
\hline DL-2-Aminooctanoic acid & 1 & 0.796434 & 0.2993 & 0.209722 & 0.401189 & 0.556145 & 0.512842 & 0.314065 & 0.693483 & 0.769051 & 0 & 0.668648 \\
\hline N-Formyl-L-methionine & 0.327368 & 0 & 0.129807 & 0.340068 & 0.266181 & 0.267696 & 1 & 0.670395 & 0.206003 & 0.470813 & 0.155567 & 0.366116 \\
\hline $\begin{array}{l}\text { N6,N6,N6-Trimethyl-L- } \\
\text { lysine }\end{array}$ & 0.184651 & 0 & 0.220002 & 0.497764 & 0.300457 & 0.210914 & 0.622371 & 0.647507 & 1 & 0.680702 & 0.28547 & 0.394471 \\
\hline $\begin{array}{l}\text { Symmetric } \\
\text { dimethylarginine }\end{array}$ & 0.28332 & 0.097123 & 0 & 0.077747 & 0.014604 & 0.185009 & 1 & 0.537427 & 0.371514 & 0.606306 & 0.399039 & 0.332586 \\
\hline Methionine sulfoxide & 0 & 0.824583 & 0.398547 & 0.333745 & 0.551494 & 0.583039 & 0.236586 & 1 & 0.195578 & 0.468576 & 0.032807 & 0.777716 \\
\hline 5-methoxy-L-tryptophan & 0.544733 & 0.415436 & 1 & 0.062012 & 0.133522 & 0.818655 & 0.019826 & 0.007316 & 0 & 0.024706 & 0.234608 & 0.035117 \\
\hline Oxidized glutathione & 0 & 0.574289 & 0.536151 & 0.333545 & 0.735102 & 0.368823 & 0.442714 & 0.433901 & 0.695535 & 0.683458 & 1 & 0.507548 \\
\hline N2-Acetylornithine & 0 & 0.36621 & 0.589176 & 0.736775 & 1 & 0.408328 & 0.633631 & 0.491555 & 0.966858 & 0.542277 & 0.403335 & 0.510622 \\
\hline 4-Guanidinobutanoic acid & 0.538393 & 0.794688 & 0.68652 & 0.835682 & 0.971408 & 1 & 0 & 0.680724 & 0.146026 & 0.126391 & 0.235248 & 0.852217 \\
\hline Prolylhydroxyproline & 0.408989 & 0.389663 & 0.404719 & 0.625843 & 1 & 0.534607 & 0.163596 & 0.208315 & 0 & 0.20809 & 0.102022 & 0.128539 \\
\hline gamma-Glutamylleucine & 0.581457 & 0.565165 & 1 & 0.478309 & 0.756397 & 0.62984 & 0.479015 & 0.366417 & 0 & 0.374885 & 0.359115 & 0.288427 \\
\hline N-Acetylleucine & 0.373575 & 0.605487 & 1 & 0.717863 & 0.468262 & 0.782234 & 0.978712 & 0.814945 & 0 & 0.87157 & 0.974042 & 0.2928 \\
\hline N-Acetylvaline & 0.655289 & 0.496573 & 0.698111 & 0.773374 & 0.835625 & 0.684023 & 0.45656 & 0.321836 & 0 & 1 & 0.661426 & 0.30436 \\
\hline $\mathrm{N}$-acetyltryptophan & 0.157182 & 0.011434 & 0.282396 & 0.386324 & 0.403771 & 0.177827 & 0 & 0.107817 & 0.209843 & 0.149415 & 1 & 0.322822 \\
\hline Alanylleucineine & 1 & 0.618709 & 0.132114 & 0.592822 & 0.95349 & 0.236102 & 0.219429 & 0.181256 & 0.221184 & 0 & 0.284367 & 0.484007 \\
\hline Gamma-Glutamyl & 0.401811 & 0.540666 & 0.304746 & 0.480864 & 1 & 0.443634 & 0.176607 & 0 & 0.105371 & 0.067001 & 0.167812 & 0.022996 \\
\hline \multicolumn{13}{|l|}{ Glutamine } \\
\hline Leucyl-Aspartate & 0.697129 & 1 & 0 & 0.005507 & 0.719274 & 0.520211 & 0.637844 & 0.649326 & 0.162156 & 0.647803 & 0.476157 & 0.220269 \\
\hline Leucyl-Serine & 0.939404 & 0.655154 & 0.488203 & 0.440834 & 1 & 0.510626 & 0.167011 & 0.132532 & 0.083535 & 0.081787 & 0 & 0.349017 \\
\hline Phenylalanyltryptophan & 0.189408 & 0.297967 & 0.875125 & 0.588916 & 0.564306 & 1 & 0.358217 & 0 & 0.04521 & 0.31401 & 0.094431 & 0.32194 \\
\hline Tyrosyl-Alanine & 0.564506 & 0.412526 & 1 & 0.057076 & 0.117832 & 0.831819 & 0.025151 & 0.1549 & 0 & 0.030541 & 0.264532 & 0.035367 \\
\hline Tyrosyl-Glutamate & 0.51936 & 0.406566 & 0.531987 & 0.726431 & 0.75 & 1 & 0.107323 & 0.123737 & 0.24032 & 0.180135 & 0.167929 & 0 \\
\hline
\end{tabular}




\begin{tabular}{|c|c|c|c|c|c|c|c|c|c|c|c|c|c|c|}
\hline $\begin{array}{l}\text { N-Methyl-1H-indole-3- } \\
\text { propanamide }\end{array}$ & 0.309741 & & 0.177889 & & 1 & 0.21482 & 0.271624 & 0.431656 & 0.558318 & 0.150077 & 0 & 0.252937 & 0.232914 & 0.495135 \\
\hline $\begin{array}{l}\text { N-gamma-L-Glutamyl-L- } \\
\text { methionine }\end{array}$ & 0.651429 & & 0.435384 & 0.40614 & & 0.817149 & 1 & 0.940203 & 0.212585 & 0.723087 & 0.022651 & 0.079565 & 0 & 0.388024 \\
\hline N-Acetylisoleucine & 0.424045 & & 0.574557 & 0.74324 & & 0.700839 & 0.350419 & 0.727866 & 1 & 0.744641 & 0 & 0.783318 & 0.581547 & 0.252563 \\
\hline Pyroglutamine & & 0 & 0.020624 & 0.00626 & & 0.365816 & 0.038971 & 0.151274 & 0.827743 & 0.585359 & 0.177807 & 1 & 0.598786 & 0.609108 \\
\hline Arg Ser Phe & $0.11030 s$ & & 0.845567 & 0.60746 & & 0 & 0.559595 & 0.584709 & 0.008603 & 0.83294 & 0.057028 & 0.912724 & 1 & 0.555155 \\
\hline Succinyl-leucyl-agmatine & 0.385676 & & 0.863135 & 0.72531 & & 0.154936 & 0.391573 & 1 & 0.151227 & 0.428557 & 0.248145 & 0.118509 & 0.020259 & 0 \\
\hline Nocardicin C & 0.258878 & & 0 & 0.47326 & & 0.317728 & 0.459868 & 0.170767 & 0.466917 & 0.412655 & 0.263599 & 0.606397 & 1 & 0.890411 \\
\hline $\begin{array}{l}\text { tert-Butyl 3-amino-1,4,6,7- } \\
\text { tetrahydro-5H- } \\
\text { pyrazolo[4,3-c]pyridine-5- } \\
\text { carboxylate }\end{array}$ & 0.37963 & & 0.863169 & 0.2242 & & 1 & 0.522634 & 0.084362 & 0.240741 & 0.594878 & 0.058642 & 0.477366 & 0.056584 & 0 \\
\hline L-2-Aminoadipic acid & & 0 & 0.112676 & 0.01072 & & 0.131029 & 0.11348 & 0.170375 & 0.690244 & 0.494896 & 1 & 0.516219 & 0.438311 & 0.124121 \\
\hline S-Glutathionyl-L-cysteine & & 0 & 0.461719 & 0.0688 & & 0.14547 & 0.264936 & 0.348534 & 0.364148 & 0.576848 & 0.140694 & 0.845685 & 1 & 0.09666 \\
\hline Metabolite Set & & total & I expected & hits & fold & $\begin{array}{c}P \\
\text { value }\end{array}$ & \multicolumn{4}{|c|}{ Up-regulated } & \multicolumn{4}{|c|}{ Down-regulated } \\
\hline $\begin{array}{c}\text { Beta Oxidation of Very Lon } \\
\text { Fatty Acids }\end{array}$ & Chain & 2 & 0.66 & 2 & 3.03 & 0.106 & \multicolumn{4}{|c|}{ 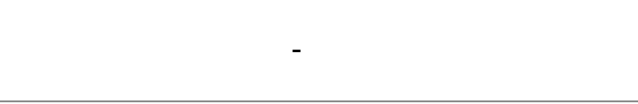 } & \multicolumn{4}{|c|}{ L-Carnitine,L-Acetylcarnitine } \\
\hline $\begin{array}{l}\text { Mitochondrial Beta-Oxida } \\
\text { Long Chain Saturated Fatty }\end{array}$ & $\begin{array}{l}\text { of of } \\
\text { Acids }\end{array}$ & 2 & 0.66 & 2 & 3.03 & 0.106 & \multicolumn{4}{|c|}{-} & \multicolumn{4}{|c|}{ L-Carnitine,Stearoylcarnitine } \\
\hline Pyruvaldehyde Degrada & & 2 & 0.66 & 2 & 3.03 & 0.106 & \multicolumn{4}{|c|}{ Glutathione,Pyruvic acid } & \multicolumn{4}{|c|}{-} \\
\hline Amino Sugar Metaboli & & 3 & 0.989 & 2 & 2.02 & 0.252 & \multicolumn{4}{|c|}{ Pyruvic acid } & \multicolumn{4}{|c|}{ L-Glutamine } \\
\hline
\end{tabular}




\begin{tabular}{|c|c|c|c|c|c|c|c|}
\hline Phosphatidylcholine Biosynthesis & 3 & 0.989 & 2 & 2.02 & 0.252 & - & Choline,O-Phosphoethanolamine \\
\hline $\begin{array}{c}\text { Phosphatidylethanolamine } \\
\text { Biosynthesis }\end{array}$ & 3 & 0.989 & 2 & 2.02 & 0.252 & - & Choline,O-Phosphoethanolamine \\
\hline Carnitine Synthesis & 6 & 1.98 & 3 & 1.52 & 0.309 & - & L-Carnitine,L-Lysine \\
\hline Glutathione Metabolism & 6 & 1.98 & 3 & 1.52 & 0.309 & Glutathione,L-Alanine & Pyroglutamic acid \\
\hline Biotin Metabolism & 1 & 0.33 & 1 & 3.03 & 0.33 & - & L-Lysine \\
\hline Glycolysis & 1 & 0.33 & 1 & 3.03 & 0.33 & Pyruvic acid & - \\
\hline Inositol Metabolism & 1 & 0.33 & 1 & 3.03 & 0.33 & D-Glucuronic acid & - \\
\hline Phenylacetate Metabolism & 1 & 0.33 & 1 & 3.03 & 0.33 & - & L-Glutamine \\
\hline Starch and Sucrose Metabolism & 1 & 0.33 & 1 & 3.03 & 0.33 & D-Glucuronic acid & - \\
\hline $\begin{array}{c}\text { Taurine and Hypotaurine } \\
\text { Metabolism }\end{array}$ & 1 & 0.33 & 1 & 3.03 & 0.33 & - & Taurine \\
\hline Aspartate Metabolism & 9 & 2.97 & 4 & 1.35 & 0.336 & L-Asparagine,L-Arginine,Citrulline & L-Glutamine \\
\hline Tryptophan Metabolism & 9 & 2.97 & 4 & 1.35 & 0.336 & L-Alanine,L-Kynurenine,Kynurenic acid & L-Tryptophan \\
\hline Urea Cycle & 12 & 3.96 & 5 & 1.26 & 0.352 & L-Alanine,Pyruvic acid,L-Arginine,Citrulline & L-Glutamine \\
\hline Fatty Acid Biosynthesis & 4 & 1.32 & 2 & 1.52 & 0.401 & - & 3-Oxohexanoic acid,3-Oxododecanoic acid \\
\hline Glucose-Alanine Cycle & 4 & 1.32 & 2 & 1.52 & 0.401 & L-Alanine,Pyruvic acid & - \\
\hline Pyrimidine Metabolism & 4 & 1.32 & 2 & 1.52 & 0.401 & - & Thymine,L-Glutamine \\
\hline Glutamate Metabolism & 11 & 3.63 & 4 & 1.1 & 0.522 & Glutathione,L-Alanine,Pyruvic acid & L-Glutamine \\
\hline Bile Acid Biosynthesis & 8 & 2.64 & 3 & 1.14 & 0.527 & - & Taurocholic acid,Taurine,Taurodeoxycholic acid \\
\hline Arachidonic Acid Metabolism & 5 & 1.65 & 2 & 1.21 & 0.536 & Glutathione,LysoPC(14:0) & - \\
\hline $\begin{array}{l}\text { Oxidation of Branched Chain Fatty } \\
\text { Acids }\end{array}$ & 5 & 1.65 & 2 & 1.21 & 0.536 & - & L-Carnitine,L-Acetylcarnitine \\
\hline Pyruvate Metabolism & 5 & 1.65 & 2 & 1.21 & 0.536 & Glutathione,Pyruvic acid & - \\
\hline Ketone Body Metabolism & 2 & 0.66 & 1 & 1.52 & 0.553 & - & (R)-3-Hydroxybutyric acid \\
\hline
\end{tabular}




\begin{tabular}{|c|c|c|c|c|c|c|c|}
\hline $\begin{array}{l}\text { Mitochondrial Beta-Oxidation of } \\
\text { Short Chain Saturated Fatty Acids }\end{array}$ & 2 & 0.66 & 1 & 1.52 & 0.553 & - & L-Carnitine \\
\hline Ammonia Recycling & 9 & 2.97 & 3 & 1.01 & 0.623 & L-Asparagine,Pyruvic acid & L-Glutamine \\
\hline Alanine Metabolism & 6 & 1.98 & 2 & 1.01 & 0.649 & L-Alanine,Pyruvic acid & - \\
\hline $\begin{array}{c}\text { Phenylalanine and Tyrosine } \\
\text { Metabolism }\end{array}$ & 6 & 1.98 & 2 & 1.01 & 0.649 & L-Phenylalanine,4-Hydroxyphenylpyruvic acid & - \\
\hline Cysteine Metabolism & 3 & 0.989 & 1 & 1.01 & 0.704 & Pyruvic acid & - \\
\hline Fatty acid Metabolism & 3 & 0.989 & 1 & 1.01 & 0.704 & - & L-Carnitine \\
\hline Histidine Metabolism & 3 & 0.989 & 1 & 1.01 & 0.704 & - & 1-Methylhistidine \\
\hline $\begin{array}{c}\text { Nicotinate and Nicotinamide } \\
\text { Metabolism }\end{array}$ & 3 & 0.989 & 1 & 1.01 & 0.704 & - & L-Glutamine \\
\hline Betaine Metabolism & 4 & 1.32 & 1 & 0.76 & 0.805 & - & Choline \\
\hline Gluconeogenesis & 4 & 1.32 & 1 & 0.76 & 0.805 & Pyruvic acid & - \\
\hline Lysine Degradation & 4 & 1.32 & 1 & 0.76 & 0.805 & - & L-Lysine \\
\hline Phospholipid Biosynthesis & 4 & 1.32 & 1 & 0.76 & 0.805 & - & Choline \\
\hline Selenoamino Acid Metabolism & 4 & 1.32 & 1 & 0.76 & 0.805 & L-Alanine & - \\
\hline Sphingolipid Metabolism & 4 & 1.32 & 1 & 0.76 & 0.805 & - & O-Phosphoethanolamine \\
\hline $\begin{array}{c}\text { Transfer of Acetyl Groups into } \\
\text { Mitochondria }\end{array}$ & 4 & 1.32 & 1 & 0.76 & 0.805 & Pyruvic acid & - \\
\hline Arginine and Proline Metabolism & 13 & 4.29 & 3 & 0.7 & 0.874 & L-Proline,L-Arginine,Citrulline & - \\
\hline Glycine and Serine Metabolism & 14 & 4.62 & 3 & 0.65 & 0.908 & L-Alanine,Pyruvic acid,L-Arginine & - \\
\hline Warburg Effect & 11 & 3.63 & 2 & 0.55 & 0.934 & Pyruvic acid & L-Glutamine \\
\hline Tyrosine Metabolism & 7 & 2.31 & 1 & 0.43 & 0.946 & 4-Hydroxyphenylpyruvic acid & - \\
\hline Citric Acid Cycle & 9 & 2.97 & 1 & 0.34 & 0.978 & Pyruvic acid & - \\
\hline Methionine Metabolism & 10 & 3.3 & 1 & 0.3 & 0.986 & - & Choline \\
\hline
\end{tabular}


